\title{
Intra-arterial growth control : slow variations on a classical theme
}

Citation for published version (APA):

Schiffers, P. M. H. (1994). Intra-arterial growth control : slow variations on a classical theme. [Doctoral Thesis, Maastricht University]. Rijksuniversiteit Limburg. https://doi.org/10.26481/dis.19940211ps

Document status and date:

Published: 01/01/1994

DOI:

10.26481/dis.19940211ps

Document Version:

Publisher's PDF, also known as Version of record

\section{Please check the document version of this publication:}

- A submitted manuscript is the version of the article upon submission and before peer-review. There can be important differences between the submitted version and the official published version of record.

People interested in the research are advised to contact the author for the final version of the publication, or visit the DOI to the publisher's website.

- The final author version and the galley proof are versions of the publication after peer review.

- The final published version features the final layout of the paper including the volume, issue and page numbers.

Link to publication

\footnotetext{
General rights rights.

- You may freely distribute the URL identifying the publication in the public portal. please follow below link for the End User Agreement:

www.umlib.nl/taverne-license

Take down policy

If you believe that this document breaches copyright please contact us at:

repository@maastrichtuniversity.nl

providing details and we will investigate your claim.
}

Copyright and moral rights for the publications made accessible in the public portal are retained by the authors and/or other copyright owners and it is a condition of accessing publications that users recognise and abide by the legal requirements associated with these

- Users may download and print one copy of any publication from the public portal for the purpose of private study or research.

- You may not further distribute the material or use it for any profit-making activity or commercial gain

If the publication is distributed under the terms of Article $25 \mathrm{fa}$ of the Dutch Copyright Act, indicated by the "Taverne" license above, 
CIP-GEGEVENS KONINKLIJKE BIBLIOTHEEK, DEN HAAG

Schiffers, Paul Maria Herman

Intra-arterial growth control: Slow variations on on a classical theme / Paul Maria Herman Schiffers. [S.I: s.n] (Voerendaal: Schrijen-Lippertz). - Ill. Thesis Maastricht. - With ref. - With a summary in Dutch. ISBN 90-9006895-3

UNGI 743

Subject headings: hypertension/intra-arterial growth control/tissue culture

Cover: Growth responses during arterial organ culture.

Production: Drukkerij Schrijen-Lippertz bv, Voerendaal. 


\section{INTRA-ARTERIAL GROWTH CONTROL}

\section{Slow variations on a classical theme}

\section{PROEFSCHRIFT}

ter verkrijging van de graad van doctor

aan de Rijksuniversiteit Limburg te Maastricht, op gezag van de Rector Magnificus, Prof. dr. H. Philipsen, volgens het besluit van het College van Dekanen, in het openbaar te verdedigen op vrijdag, 11 februari 1994 om 14.00 uur

door

Paul Maria Herman Schiffers

geboren te Heerlen in 1957 


\section{Promotor}

Prof. dr. H.A.J. Struijker Boudier

Co-promotor

Dr. J.G.R. De Mey

Beoordelingscommissie

Prof. dr. P.W. de Leeuw, voorzitter

Dr. M.J.A.P. Daemen

Prof. dr. A.C. Grittenberger-de Groot, Rijksuniversiteit Leiden

Prof. dr. A.M. Heagerty, University of Manchester

Prof. dr. P.J.H.M. Kitslaar

Financial support by the Netherlands Heart Foundation and the Dr. Ir. van de Laar Foundation for the publication of this thesis is gratefully acknowledged. 
Alleen voor jullie, zonen van wetenschap en wijsheid, hebben wij dit werk geschreven. Studeert aandachtig in dit boek en zoekt onze bedoelingen bijeen, die wij verspreid over verschillende plaatsen hebben uiteengezet; en wat op eén plaats door ons verborgen is gehouden, hebben wij op een andere duidelijk gemaakt, zodat het voor jullie onthuld wordt, als jullie wijs zijn.

Heinrich Cornelius Agrippa von Nettesheim, De Occulta Philosopia, III, 65. 


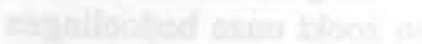

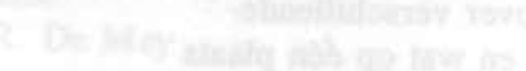

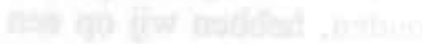

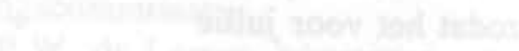

the

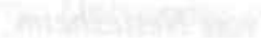

20.717. 


\section{CONTENTS}

1. Introduction 1

2. Materials and Methods 29

3. DNA synthesis in isolated arteries. Kinetics and structural 43 consequences

4. Growth responses in isolated elastic, muscular and resistancesized arterial segments of the rat

5. DNA synthesis in isolated arteries of normotensive and hypertensive rats: Effects of the endothelium

6. Effects of candidate autocrine and paracrine mediators on growth responses in isolated arteries of the rat

7. In vivo DNA synthesis is not uniformly increased in arterial smooth muscle of young spontaneously hypertensive rats

8. Heterogeneity of growth responses in the arterial system of young rats during vasoconstrictor infusion

9. Tonic tone in arteries continuously exposed to angiotensin II

10. Conclusions and remarks

References

Summary

Samenvatting

Curriculum Vitae

List of publications

Dankwoord 


\section{LIST OF ABBREVIATIONS}

\begin{tabular}{|c|c|}
\hline $\mathrm{ACE}$ & angiotensin-converting-enzyme \\
\hline Ang II & angiotensin II \\
\hline $\mathrm{AO}$ & thoracic aorta \\
\hline ASMC & arterial smooth muscle cells \\
\hline bFGF & basic fibroblast growth factor \\
\hline $\mathrm{BQ} 123$ & endothelin ETa-receptor antagonist \\
\hline BrdUrd & 5-bromo-2'-deoxyuridine \\
\hline $\mathrm{CA}$ & carotid artery \\
\hline CMS-PDS & PDS stripped from progression factors \\
\hline CSA & cross sectional area \\
\hline dFCS & dialyzed fetal calf serum \\
\hline $\mathrm{ET}-1$ & endothelin-1 \\
\hline FA & femoral artery \\
\hline $5 \mathrm{HT}$ & serotonin \\
\hline IL-1 & interleukin-1 \\
\hline KRB & Krebs-Ringer bicarbonate solution \\
\hline L-arg & L-arginine \\
\hline L-NAME & $\mathrm{N}^{\mathrm{G}}$-nitro-L-arginine methyl ester \\
\hline MA & superior mesenteric artery \\
\hline MrA & mesenteric resistance-sized artery \\
\hline NO & nitric oxide \\
\hline PCNA & proliferating cell nuclear antigen \\
\hline PDGF & platelet-derived growth factor \\
\hline PDS & plasma-derived serum \\
\hline PHE: & phenylephrine \\
\hline RA & renal artery \\
\hline RAS & renin-angiotensin system \\
\hline $\operatorname{Rr} A$ & renal resistance-sized artery \\
\hline Sar & saralasin \\
\hline SDR & Sprague-Dawley rats \\
\hline SHR & Spontaneously hypertensive rats \\
\hline SNP & sodium nitroprusside \\
\hline WIS & Wistar rat \\
\hline WKY & Wistar-Kyoto rats \\
\hline TGR & transgenic hypertensive rats (mouse Ren-2 gene) \\
\hline TGF-B & transforming growth factor $B$ \\
\hline
\end{tabular}




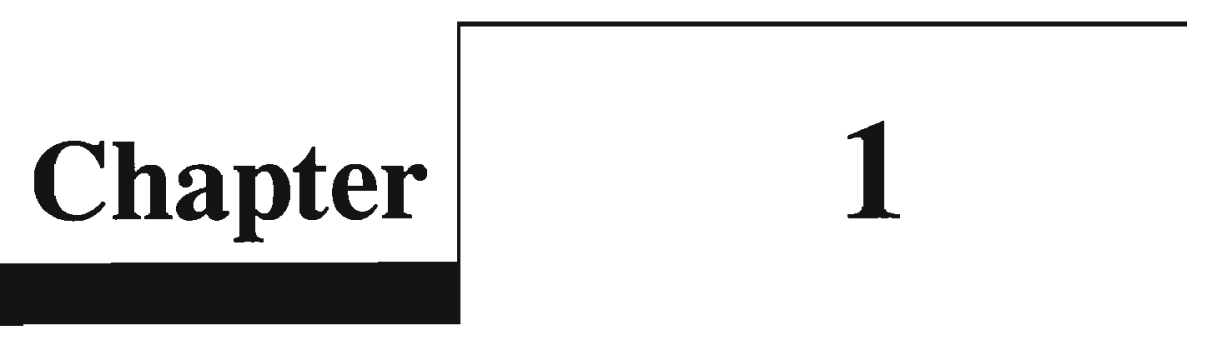

Introduction 


\section{HYPERTENSION}

\section{Definition and importance}

Hemodynamically, blood pressure is a reflection of the amount of blood pumped by the heart per minute (cardiac output) and the ease with which the blood flows through the peripheral vasculature (vascular resistance). Because in all forms of chronic hypertension cardiac output is within a normal range, the hemodynamic hallmark of hypertension lays within the peripheral resistance (Sivertsson, 1970; Folkow,1982, 1990; Lund-Johansen, 1980, 1983). It is disturbing that the etiology of most hypertension remains unknown: about $95 \%$ of the patients are considered to be suffering from primary or essential hypertension since no specific cause has been identified for their condition (Epstein, 1983; Julius and Hansson, 1983).

Elevated blood pressure is recognized to constitute the most important risk factor for secondary cardiovascular disorders (Stamberg et al, 1972; MacMahon et al, 1990). Most of these are due to the fact that atherosclerosis develops earlier and more extensively in hypertension. Current antihypertensive treatments are highly successful in reducing blood pressure, primarily by interference with acutely acting pressor stimuli, but far less successful in reducing morbidity and mortality due to hypertension-induced cardiovascular disorders (MacMahon et al, 1986; Lindholm et al, 1984; Isles et al, 1986). Both hypertension and atherosclerosis are characterized by structural changes in the vascular wall (Folkow, 1990; Bondjers et al, 1991; Dahlhöf, 1988; Schwartz and Reidy, 1987). These are refractory to at least some forms of antihypertensive therapy (Struijker-Boudier et al, 1990; Mulvany, 1990). Better understanding of the mechanisms of vascular structural changes in hypertension and atherosclerosis may contribute to the development of treatments that not only involve a reduction in blood pressure, but also regression or prevention of structural vascular changes.

\section{Control of blood pressure}

In the circulation, total vascular resistance can be approximated by Poisseuille's law for a vascular network:

$$
R=\frac{1}{N} \cdot \frac{8 \eta L}{\pi r^{4}}
$$

where $\mathrm{N}$ is the number of vessels involved, $\mathrm{r}$ the radius of the vessel, $\mathrm{L}$ the 
length of the vessel, $\eta$ the viscosity of the fluid. Since the radius is expressed as the fourth power in the equation, flow and pressure may be markedly affected by relatively small changes in vessel diameter. The basic relationship between arterial pressure, cardiac output, and "total peripheral resistance" (TPR) is given by:

$$
M A P=C O \times T P R
$$

where CO is cardiac output, MAP is mean arterial pressure and TPR is total peripheral resistance. The relationship $\mathrm{MAP}=\mathrm{CO}$. TPR states the basic determinants of arterial pressure and provides a framework for understanding the influences of various factors that control pressure and that are of importance for hypertension.

\section{Pathogenesis of essential hypertension}

Important information concerning the pathogenesis of hypertension has been derived not only from studies in man, but also through the development of experimental models of hypertension. Hypertension has been induced in laboratory animals, primarily rats, by a range of interventions and by selective inbreeding (table 1.1). Some of them will be discussed in this introduction and used in the experimental approaches of this thesis.

In almost all clinical and experimental forms of chronic hypertension, blood pressure is maintained by an elevated peripheral vascular resistance. Over the years, three main theories have been proposed in this respect: (1) hyperactivity of sympathetic nervous system; (2) changes in kidney function; and (3) functional and structural alterations of the arterial wall, including vascular smooth muscle hyperreactivity and hypertrophy of the vascular wall (fig.1.1). Other factors which can be broadly classified as genetic and environmental, contribute as risk factors (Laurenzi et al, 1990). These include diabetes, obesity, high dietary salt intake, stress, heredity and aging.

Many papers have emphasized that sympathetic nervous hyperactivity may be a key factor in the development of hypertension (Abboud, 1984; Beilin et al, 1983; Scott and Galway, 1985; Goldstein, 1983; Egan et al, 1987; Izzo et al, 1987; Judy et al, 1976; Collis et al, 1979, 1980). A sustained increase in sympathetic outflow could have many consequences that ultimately lead to increased blood pressure. These include increased release of norepinephrine from peripheral vascular nerve endings activating adrenoceptors on blood vessels, the release of catecholamines from the adrenal medulla, the release of renin from juxtaglomular cells and the consequential multiple actions of 
Table 1.1 Rat experimental models of hypertension.

$\begin{array}{lll}\text { Model Control Characteristics } & \end{array}$

\begin{tabular}{|c|c|c|}
\hline $\begin{array}{l}\text { Spontaneously } \\
\text { Hypertensive } \\
\text { rat (SHR) }\end{array}$ & Wistar-Kyoto & $\begin{array}{l}\text { early increased } \\
\text { sympathetic activity }\end{array}$ \\
\hline Dahl-sensitive & Dahl-resistant & $\begin{array}{l}\text { hypertensive on } \\
\text { exposure to high } \\
\text { salt diet }\end{array}$ \\
\hline $\begin{array}{l}\text { Transgenic } \\
\text { rats (TGR) }\end{array}$ & Sprague-Dawley & $\begin{array}{l}\text { introduction mouse } \\
\text { Ren- } 2 \text { gene }\end{array}$ \\
\hline Goldblatt $2 \mathrm{~K}-1 \mathrm{C}$ & - & $\begin{array}{l}\text { renin } \uparrow \text { then normal; } \\
\text { sodium balance } \downarrow \text { or } \\
\text { normal }\end{array}$ \\
\hline Goldblatt $1 \mathrm{~K}-1 \mathrm{C}$ & - & $\begin{array}{l}\text { renin } \downarrow \text { or normal; } \\
\text { sodium balance } \uparrow\end{array}$ \\
\hline DOC-salt & - & $\begin{array}{l}\text { renin } \downarrow \text {; sodium } \\
\text { balance } \uparrow\end{array}$ \\
\hline Aortic coarctation & - & $\begin{array}{l}\text { renin } \uparrow \text { then normal; } \\
\text { sodium balance? }\end{array}$ \\
\hline
\end{tabular}

angiotensin-II (Ang II) (Shepherd, 1990).

Several investigators have focussed on an important role of the kidney in the initiation of the increase in vascular resistance as observed in hypertension. The two main concepts that have been elaborated in this respect are: i) an impaired salt and water excretion and ii) an increased renin secretion. If urine excretion is impaired, blood pressure will rise and the increased blood pressure restores urine output at the expense of hypertension (Guyton et al, 1986). Such a shift of the renal function curve could occur as the result of a genetic abnormality in kidney function, an increased sympathetic nervous activity or the action of Ang II and aldosterone. Renin-dependent hypertension in man is associated with some forms of secondary hypertension, such as in renin-secreting tumors and renal artery stenosis (Wilson and Byrom, 1939; 


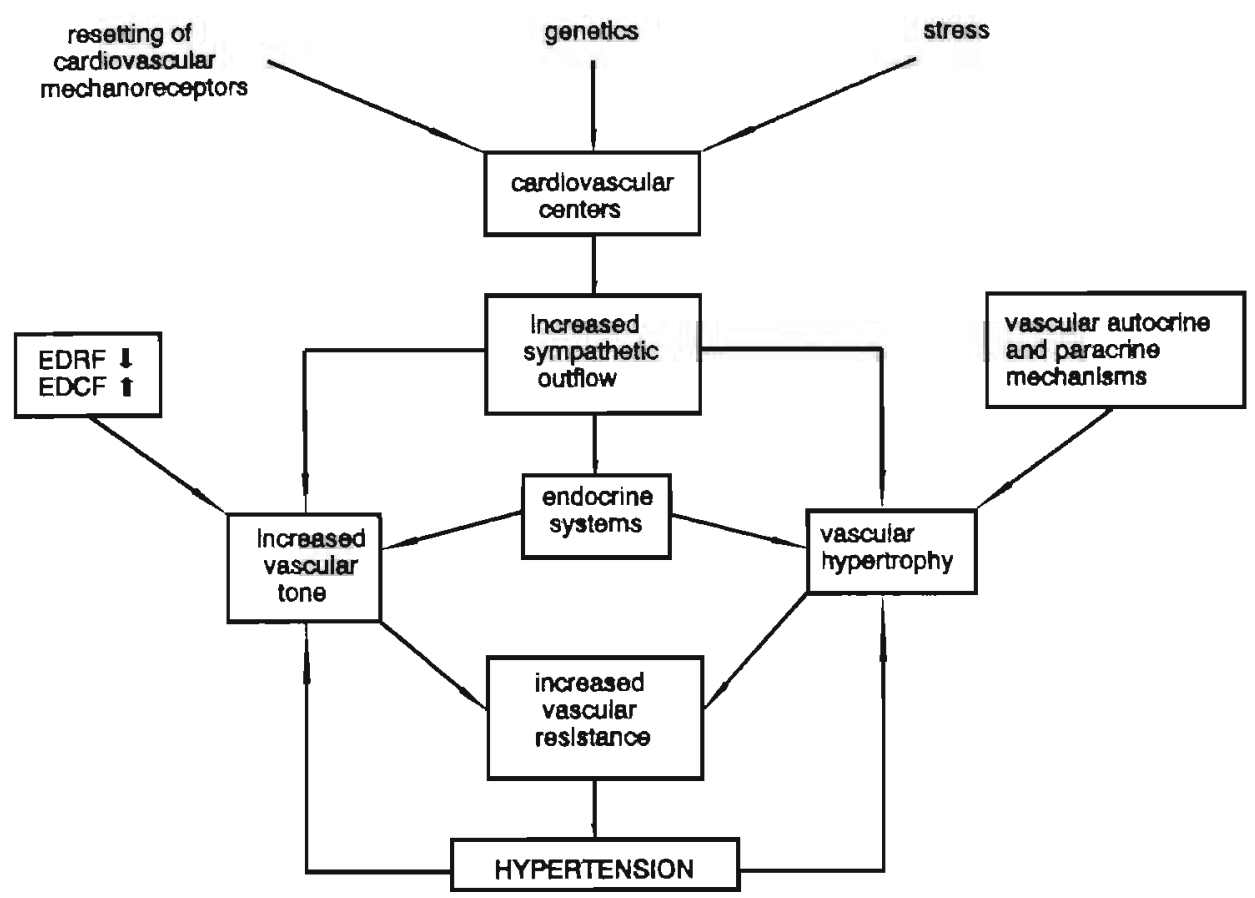

Figure 1.1 Mechanisms involved in total peripheral resistance (TPR) elevation in hypertension. $(E D C F=$ endothelium derived contracting factors; $E D R F=$ endothelium derived relaxing factors).

Goldblatt et al, 1984). The latter changes in the renin-angiotensin systen (RAS) can be simulated in the 2-kidney 1-clip or 1-kidney 1-clip rat models of hypertension (table 1.1). In these models the RAS is involved in the initiation of increased blood pressure by the acute vasopressor action of Ang II (Bing et al, 1981). The mechanisms that maintain the elevated blood pressure in the chronic phase are not entirely clear. They may involve sodium and water retention and structural vascular changes (Guyton et al, 1986; Folkow, 1982, 1990).

Functional changes of the vascular wall may also contribute in the development of increased blood pressure. There are a number of reports arteries from spontaneously hypertensive rats (SHR) in vitro, having an increased sensitivity to contractile substances (Lais and Brody, 1978; Webb, 1984; Triggle, 1989). This increased sensitivity is suggested to be a result of 
an altered receptor affinity for the particular substance (Michel et al, 1989, 1990), a change in receptor density (number) (Abboud, 1982) or an alteration in receptor activity (Uehara et al, 1988; Koutouzov et al, 1987). An increased receptor affinity in hypertension has been observed for noradrenaline, serotonin and Ang II (Nyborg and Bevan, 1988; Collis and Vanhoutte, 1977; Lundin et al, 1984; De Mey and Vanhoutte, 1981 ${ }^{\mathrm{a}}$ ). Furthermore, it has been suggested that vascular smooth muscle plasma membrane systems (ion channels, ionexchangers and active pumps) involved either directly or indirectly in the regulation of intracellular ionized calcium concentrations, are abnormal in hypertension (Hermsmeyer, 1984; Webb, 1984; Dominiczak and Bohr, 1989; Aalkjaer, 1990; Postov, 1990). In addition, SHR small arteries in vitro have been described to show an increased sensitivity to ionized calcium (Mulvany and Nyborg, 1980; Boonen and De Mey, 1990).

The functional importance of endothelial cells as regulators of cardiovascular function has been increasingly recognized (Furchgott and Vanhoutte, 1989; Lüscher, 1988). Endothelial cells produce vasoactive factors which can inhibit or stimulate contractile reactivity of vascular smooth muscle. Under physiological conditions endothelium-derived relaxing factors appear to dominate. In a variety of experimental models of hypertension, the responsiveness of isolated large and small arteries to acetylcholine and other endotheliumdependent relaxing agents are attenuated (Lockette et al, 1986; De Mey and Gray, 1985; Lüscher and Vanhoutte, 1986; Winquist et al, 1984; Lüscher et al, 1987). The role of endothelium-derived contracting factors, on the other hand, appears to become more prominent, leading to an imbalance of endothelium-dependent vascular regulation (Vanhoutte, 1989; Lüscher et al, 1992). This imbalance of endothelium-derived relaxing and contracting factors may be important in the pathogenesis of hypertension and its cardiovascular complications.

While functional differences in vascular smooth muscle reactivity have been described (hyperreactivity, see above), and may account for some of the elevated resistance in hypertension, vascular structure also participates in the resistance defect. The importance of structural changes is demonstrated by i) the observation that different vascular beds in essential hypertension, and animal models of hypertension (SHR) show a higher flow resistance under conditions of maximal dilatation induced either pharmacologically or by reactive hyperaemia (Folkow, 1982; Sivertsson, 1970), ii) the presence of structural alterations in blood vessels prior to the development of hypertension (Lee and Smeda, 1985; Lee, 1985; Smeda et al, 1988 ; Gray, 1984) and iii) the effects of various antihypertensive therapies on vascular structure in essential and experimental hypertension (see chapter: current antihypertensive therapy and vascular structure)(Struijker-Boudier, 1990; Mulvany, 1990). These observations indicate that some structural alterations of the vessel wall may be 
primary changes contributing to the development of hypertension. Recent evidence suggests that both the sympathetic nervous system and the endothelium may also be involved in the regulation of vascular structure by trophic influences on vascular smooth muscle (fig. 1.1)(de Champlain, 1990; Kishi and Inoue, 1990; Blaes and Bloissel, 1983; Yamori et al, 1987; Josheph-Silverstein and Rifkin, 1987; Davies, 1986; DiCortello et al, 1983). In the following chapters of this introduction, I will further concentrate on structural alterations of the arterial vessel wall in hypertension and atherosclerosis.

\section{VASCULAR STRUCTURE}

\section{Hemodynamic relevance}

Total vascular resistance is according to Poisseuille's law determined by three structural factors: (a) the internal diameter of the blood vessel, (b) the length of the vessel and (c) the number of vessels. In hypertension there is a decrease in internal vascular diameter. Because the internal radius is present in the Poisseuille's law to the fourth power, small changes in this variable will have profound effects on vascular resistance. The other structural alteration in hypertension that has been proposed to contribute to increased resistance is rarefaction of arterioles and capillaries (reduction in number of vessels) (Hutchins and Darnell, 1974; Prewitt et al, 1982; Le Noble et al, 1986). This factor is present in the equation only to the first power.

\section{Morphogenesis}

The vascular system is an elaborated network of a number of vascular segments coupled in series and in parallel (fig.1.2). Recent findings highlight the importance of genetic factors with respect to the architecture of the vascular system (Noden, 1989). Long recognized hemodynamic forces (pressure and flow; wall stress and flow shear rate)(Glagov et al, 1992; Folkow, 1990) and metabolic factors (Mohrman, 1991) along with more recently recognized growth and angiogenic factors (Adair et al, 1990) would act on the top of this predetermined frame during normal embryogenesis and development (Lever and Harrap, 1992).

A blood vessel consists of but three cell types: endothelial cells, smooth muscle cells and fibroblasts. They are organized in three distinct structures, the intima, the media and adventitia, respectively. The vascular tree is a circuit that conducts blood from the heart through large diameter, low-resistance conducting "wind-kessel" vessels to small arteries and arterioles. The 


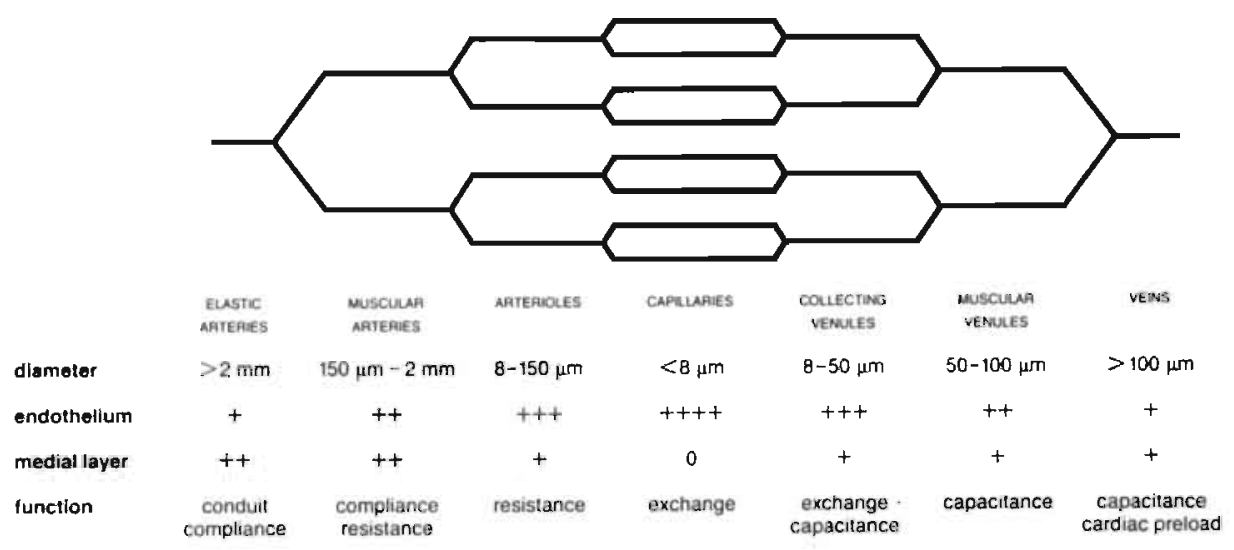

Figure 1.2 The serially and parallel coupled segments in the vascular tree. For each segment, average diameter, relative contribution of the endothelium and media, and major physiological functions are indicated. Reproduced from Struijker-Boudier et al (1990) with permission.

capillaries are thin-walled and allow the exchange of nutrients and waste products between tissue and blood, a process that requires a very large surface area. The circuit back to the heart is completed by the veins, which are distensible and provide a volume buffer that acts as a capacitance for the vascular circuit. These segments all differ in their relative number of the three cell types and the presence of extracellular matrix components (fig.1.2).

Besides the structural differences between vascular segments of different anatomical location, there are also marked heterogeneities in the functional properties of blood vessels (Vanhoutte, 1978; De Mey and Vanhoutte, 1981ª, 1982; Heesen and De Mey, 1990). This functional heterogeneity is expressed by differences in the degree of importance of contractile factors, the degree of innervation, the distribution of receptors for pressor and depressor hormones, the second messenger systems involved, and the differential control exerted by endothelium-derived mediators. This, again, shows how complex vascular resistance is regulated, besides the many other factors that are involved. 


\section{Vascular structure in human essential hypertension and experimental hypertension}

As in essential hypertension in man, the development of increased blood pressure in experimental hypertension is associated with an increased wall-tolumen ratio. This increased wall to-lumen-ratio can be obtained by i) an increase in arterial smooth muscle mass (Folkow, 1982; Schwartz et al, 1986; Lever, 1986) and ii) structural narrowing of the arterial wall in the absence of significant increase in media mass (remodeling)(fig.1.3). Depending on the anatomical location of the vessel the nature of vascular hypertrophy in hypertension includes vascular smooth muscle cell hyperplasia, -hypertrophy, and -hyperploidy (Owens, 1989). The former predominates in small resistancesized arteries while hypertrophy and hyperploidy have been observed in muscular and elastic arteries (Aalkjaer et al, 1987 ; Mulvany et al, 1985; Owens and Schwartz, 1982, Owens, 1985; Kosgaard and Mulvany, 1988; Owens et al, 1988 ; Black et al, 1989).

In culture conditions without the influence of arterial pressure and other stimuli several studies have demonstrated that aortic smooth muscle cells from SHR have an increased proliferative activity (Pang, 1989; Paquet et al, 1989; Hadrava et al, 1989; Scott-Burden et al, 1989 $)$. This occurrence indicates an intrinsic genetic abnormality in the growth control of vascular smooth muscle from SHR. In addition, structural alterations in small arteries in vivo are present at the prehypertensive phases (Lee, 1985; Gray, 1984), and these changes are little influenced by antihypertensive treatment (Mulvany, 1990; Nyborg and Mulvany 1985; Sano and Tarazi, 1987)(see chapter: current antihypertensive therapy and vascular structure). These studies suggest that vascular smooth muscle hyperplasia may be causally related to essential hypertension (Kanbe et al; 1983; Lever, 1986; Mulvany, 1986).

In contrast, there is evidence that smooth muscle cell hypertrophy and hyperploidy may be a response to increased blood pressure. Many studies consistently observed a linear correlation between the level of blood pressure and the frequency of polyploidy in a variety of hypertensive models (Chobanian et al, 1987; Lichtenstein et al, 1986; Owens, 1985, 1987; Owens and Schwartz, 1982, 1983). The development of smooth muscle cell hypertrophy and hyperploidy in aortas of spontaneously hypertensive rats occurs predominantly after blood pressure has increased to its maximal level (Owens and Schwartz, 1982, 1983). The normalization of blood pressure in spontaneously hypertensive rats treated with multiple antihypertensive drugs is effective in preventing further development of smooth muscle cell hypertrophy and hyperploidy as well as in reversing cellular hypertrophy.

Although many factors have been identified that influence growth of cultured vascular smooth muscle cells, the precise mechanisms that mediate 


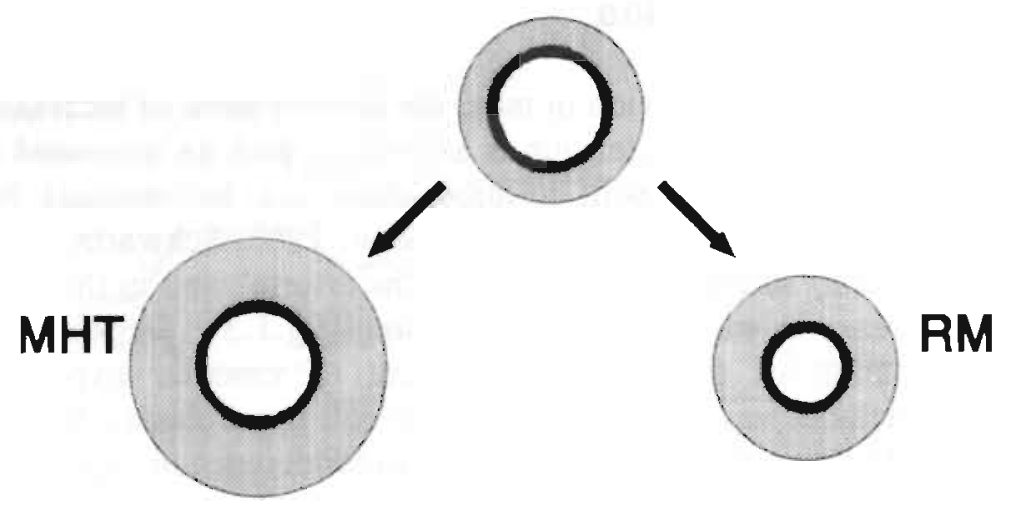

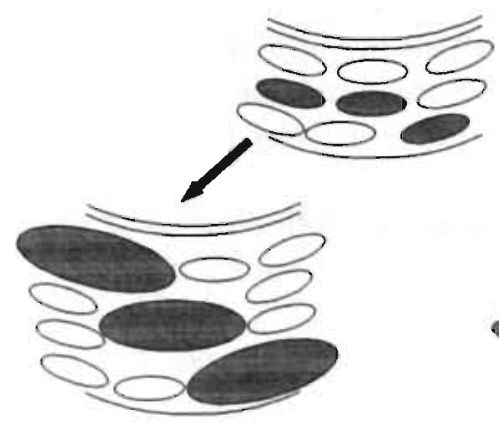

$\mathrm{CHT}$

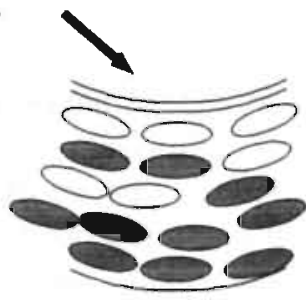

$\mathrm{HP}$

Figure 1.3 Growth responses of the vascular tree in hypertension. The wall to lumen ratio is altered by an increase in smooth muscle mass (media hypertrophy; MHT) or structural remodeling without increase in smooth muscle mass (RM). MHT is either a result of larger smooth muscle cells (cellular hypertrophy; $\mathrm{CHT}$ ) or increased number of medial cells (HP).

hypertrophic versus hyperplastic growth of smooth muscle cells in vivo have not been defined, nor is it clear how the controls for these two processes are related (Owens, 1989). Also very little is known regarding the potentially different functional implications of increased smooth muscle mass due to cellular hypertrophy or hyperplasia. 


\section{Structural responses to hypertension and atherosclerosis: a common underlying mechanism?}

Not only hypertension but also atherosclerosis is characterized by trophic changes in the vascular wall. In atherosclerosis intimal hyperplasia develops in large blood vessels and leads to reduced flow and clinical symptoms of ischemia. Though seemingly different diseases, recent insights suggest that comparable cellular and molecular mechanisms may be involved in the initial stages of plaque formation and in hypertensive vascular changes. Different microenvironments and different initiating stimuli act upon essentially the same process. At large, mechanisms of atherogenesis are better understood (Bondjers et al, 1991; Schwartz and Reidy, 1987; Schwartz, 1984; Schwartz et al, 1986). They will be reviewed below and their relevance for hypertension will be briefly discussed.

The most widely discussed model to explain the pathogenesis of atherosclerosis is the "response to injury" hypothesis (Clowes et al, 1983a; Ross and Glomset, 1985). It is postulated on the basis of experiments in which experimental mechanical injury of the arterial wall leads to intimal thickening with proliferating smooth muscle cells (Bondjers and Björnheden, 1970; Stemerman and Ross, 1973; Björkerud and Bondjers, 1973; Ross and Glomset, 1985; Björkerud and Bondjers, 1976). The model suggests that initial endothelial injury or dysfunction leads to intimal vascular smooth muscle cells migration and proliferation, that is accompanied by infiltration of inflammatory cells, connective tissue formation and lipid deposition (Ross and Glomset,1976; Ross, 1986). The sites at which atherosclerosis develops are characterized by increased hemodynamic strain. In particular, deviations from the normal shear stress exerted by blood flow appear to be connected with endothelial injury (Davies et al, 1986; Langille et al, 1986).

Because approximately $2 / 3$ of the atherosclerotic plaque volume is constituted by smooth muscle cells and smooth muscle cell synthetic products, intimal migration and proliferation of vascular smooth muscle cells is an important phenomenon in the atherogenic process (Stemerman and Ross, 1973; Ross and Glomset, 1985; Björkerud and Bondjers, 1976). It is not clear whether the increase in smooth muscle cells represents large numbers of migrant medial cells (phenotypic transition, contractile into synthetic) or arise from rapid division of medial stem cells (Chamley-Campbell et al, 1979; Thyberg et al, 1983; Fager et al, 1989; Moss et al, 1985; Benditt and Benditt, 1973; Schwartz et al, 1986).

The involvement of immunocompetent cells in the atherogenic process has emphasized that local inflammation, too, may be an important component of the response to injury (Jonassen et al, 1986; Hansson et al, 1989; Libby and Hansson, 1991). T lymphocytes and macrophages are abundant in the 
atherosclerotic lesions (Munro and Cotran, 1988), and their secretory products have profound effects on endothelial cells (Luscinskas et al, 1991) and vascular smooth muscle cells (Shimokade et al, 1986; Libby et al, 1988; Assoian et al, 1987; Owens et al, 1988'; Stemme et al, 1990). Both growth promoting and growth inhibiting factors may be involved.

In addition, numerous investigations have emphasized a role for extracellular matrix components in the control of vascular smooth muscle proliferation (Thyberg et al, 1990; Majack et al, 1986; Scott-Burden and Bühler, 1988). For instance, under normal conditions the basement membrane is believed to maintain vascular smooth muscle cells in a contractile state. Injury to this sheet-like network is suggested to initiate a transition of the cells to a synthetic phenotype (Thyberg et al, 1990). Other factors that may influence growth of vascular smooth muscle cells, even in the absence of injury, include noradrenaline and other neurotransmitters, mechanical factors, and contractile agonists from circulating blood, from endothelial cells, or generated by smooth muscle cells themselves (Owens, 1989).

All these above mentioned endocrine, paracrine and autocrine mechanisms may have a function in modulating the smooth muscle phenotype from a contractile state into a proliferative state during atherogenesis. In the monoclonal hypothesis, on the other hand, one or more of these factors could activate medial stem cells to start proliferating during the course of the atherogenic process. Although there is experimental support for both models by which vascular smooth muscle cells could proliferate, more work is needed to fully clarify the pathogenesis of atherosclerosis.

The relation between hemodynamic strain and localization of atherosclerotic plaques provides one potential explanation of the relation between hypertension and atherosclerosis. However, it is obvious that such a view of the relation between these processes may represent an oversimplification. The possibility that both diseases contain an abnormal growth control in the smooth muscle cell population suggests that susceptibility to atherogenic stimuli may be determined by the same factors that control the regulation of the blood pressure.

\section{Structural mechanisms in hypertension}

The classical model that describes the structural mechanisms involved in the pathogenesis of hypertension was suggested by Folkow in the 1950s. Overactivity of a pressor mechanism causes a small increase in pressure. The resulting vascular hypertrophy amplifies the pressor signal by a positive feedback, producing further hypertrophy in a vicious circle (fig 1.4). The initiating cause may be small, quick-acting and specific for a certain form of hypertension, while the amplification is slowly progressive and non-specific. 


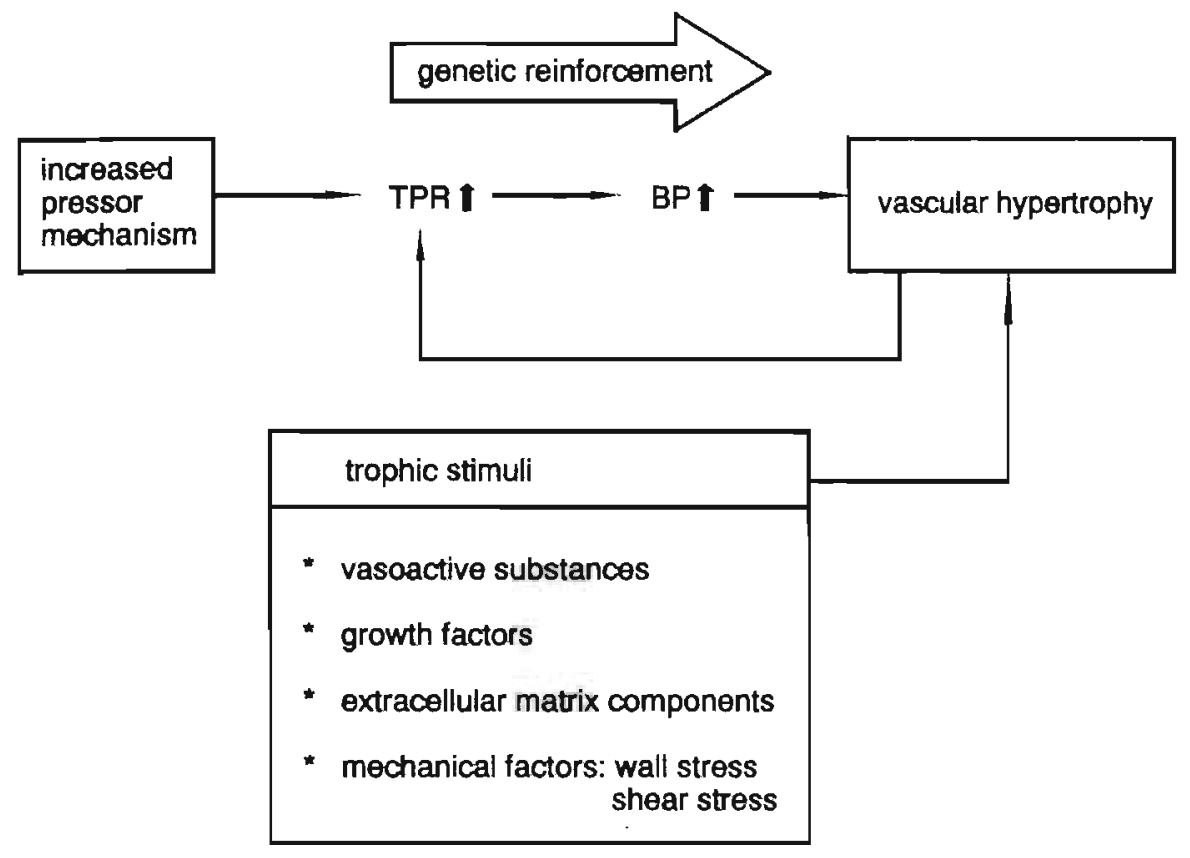

Figure 1.4 Slow pressor mechanisms in hypertension. Adapted from Lever (1986).

This model has been extended by the introduction of slow pressor stimuli (Lever, 1986), suggesting that alterations in vascular structure are not only a secondary adaption to high blood pressure, but that some alterations in the cardiovascular structure may be primary changes related to the development of hypertension (fig 1.4).

As already mentioned, most of the current knowledge about the factors that influence vascular smooth muscle proliferation are derived from the vascular injury model and cell culture experiments. Below, I will discuss a possible role for some of these factors in the pathogenesis of hypertension. Attention will be paid to vasoactive substances, polypeptide growth factors and extracellular matrix components in regulating vascular smooth muscle cell growth (fig.1.4). 


\section{Vasoconstrictors}

The idea that contractile agonists may play a direct role in the mediation of smooth muscle cell growth is intriguing, since it would provide a mechanism whereby vascular smooth muscle mass could be altered in response to vasoconstrictor input. This mechanism may not be limited to growth responses in hypertension but may also occur as a physiological response during normal vessel growth and development. Furthermore, this response could be important in the normal maintenance of vascular smooth muscle mass. Because hyperreactivity of the sympathetic nervous system and the renin-angiotensinaldosterone system have been suggested to participate in the development of hypertension, it is of interest that a number of contractile agonists including catecholamines and Ang II, but also other factors such as endothelin-1 (ET-1), vasopressin and serotonin, have been shown to have growth promoting activity in vascular smooth muscle.

Ang II has been shown to stimulate protein and DNA synthesis, protooncogene expression (c-fos and c-myc), as well as PDGF A-chain expression in cultured vascular smooth muscle (Naftilan et al, 1989; Scott-Burden et al, 1988, 1991; Berk et al, 1989ª Kawahara et al, 1988; Turla et al, 1991; Geisterfer et al, 1988; Schelling et al, 1991).

Endothelin-1 has been reported to stimulate the expression of protooncogenes (c-fos and c-myc) and the elevation of DNA-synthesis in isolated vascular smooth muscle cells (Komuro et al, 1988; Dubin et al, 1989; Bobik et al, 1990; Hirata et al, 1989). Exposure of vascular smooth muscle cells to Ang II, vasopressin or PDGF AA leads to the induction of ET-1 secretion, and this may result in an autocrine growth stimulation (Resink et al, 1990).

Studies on subcultured vascular smooth muscle cells have indicated that catecholamines like epinephrine and norepinephrine, stimulate proliferation of vascular smooth muscle cells in the presence of serum and that the effect is mediated via $\alpha$-adrenergic receptors (Blaes and Bloissel, 1983; Bauch et al, 1987; Nakaki et al, 1990; Kishi and Inoue, 1990). Stimulation of both protein synthesis and DNA synthesis in cultures of vascular smooth muscle cells by arginine vasopressin has also been reported (Scott-Burden et al, 1989; Campbell-Boswell and Robertson, 1981; Geisterfer and Owens, 1989; Turla et al, 1991). In addition, serotonin was likewise observed to produce a mitogenic effect in cultured aortic vascular smooth muscle cells (Lee et al, 1991; Nemecek et al, 1986).

In general, contractile agonists serve as partial growth factors for smooth muscle cells, initiating the increased cell mass associated with cell cycle progression and in some cases DNA replication but not cellular division. These contractile agonists might therefore stimulate cellular hypertrophy rather than hyperplasia. 


\section{Growth factors}

Platelet-derived growth factor (PDGF), a cationic protein composed of two polypeptide chains made up of combinations of so-called $A$ and $B$ chains, is a potent mitogen in vitro for vascular smooth muscle cells (Ross et al, 1974, 1978, 1986; Williams, 1989; Westermark et al, 1989; Bowen-Pope and Ross, 1982; Weinstein et al, 1981; Nilson et al, 1983). Studies suggest that PDGF is the most potent inducer of endogenous PDGF production, although other growth factors (Ang II and TGF- $B$ ) may have a similar effect (Naftilan et al, 1989; Majack et al, 1990). Furthermore, $\alpha$-adrenergic agonists stimulate in vivo the expression of the PDGF A-chain gene as well as other growth-related genes in rat aortic smooth muscle (Majesky et al, 1990). In addition to its effects on cellular replication, PDGF has been found to stimulate migration of smooth muscle cells as well as inflammatory cells (Williams, 1989; Westermark, 1989; Grotendorst et al, 1982). Beside the mitogenic effect of PDGF, this polypeptide growth factor induces contractions in isolated rat aortic strips (Berk et al, 1986; Berk and Alexander, 1989).

Other polypeptide growth factors are EGF and FGF (Schlessinger et al, 1983; Burgess and Maciag, 1989; Klagsbrun and Edelman, 1989). Vascular smooth muscle cells have receptors for and respond mitogenically to both of these factors (Scott-Burden et al, 1989 ${ }^{\text {; }}$ Winkels et al, 1987; Gospodarowicz et al, 1981). EGF can also function as a vasoconstrictor through stimulation of prostaglandin production (Berk et al, 1986). Human and bovine smooth muscle cells have also been found to express and respond to acidic and basic FGF, respectively (Winkles et al, 1987; Gospodarowicz et al, 1988). Moreover, aFGF and bFGF have been found to induce a hypotensive effect in vivo after administration to anesthetized rats. (Cuevas et al, 1991). Unlike PDGF, the genomic structure of FGF demonstrates that there is no signal sequence in the molecule, which suggests that their extracellular translocation may be mediated via a carrier/binding molecule (Gimenez-Gallego et al, 1985) or that their "secretion" may repair cellular injury (d'Amore, 1987).

Attention has also been paid to interleukin-1 (IL-1) (Martin and Resch, 1988) and TGF-ß (Sporn et al, 1987). IL-1 was found to stimulate smooth muscle proliferation (Libby et al, 1988; Bonin et al, 1989) apparently via induction of endogenous PDGF production (Raines et al, 1989). Human smooth muscle cells were shown to produce IL-1 when exposed to bacterial endotoxin or IL-1 (Libby et al, 1986; Warner et al, $1987^{\circ}$ ). Recently, a growth-promoting effect of interleukin-6, a cytokin secreted by monocytes and macrophages, has also been reported (Nabata et al, 1990). Moreover, smooth muscle cells stimulated with IL-1 or PDGF secrete large amounts of IL-6 (Loppnow and Libby, 1990). With regard to TGF- $B$, both inhibitory and stimulatory effects have been reported (Owens et al, 1988' ; Assoian and Sporn, 1986; Majack, 1987). TGF- $\beta$ inhibits serum- or PDGF-stimulated proliferation of vascular 
smooth muscle cells at a low cell density and potentiates it at a high cell density (Majack, 1987; Goodman and Majack, 1989). PDGF and epidermal growth factor elevate the expression of TGF- $\beta$, which also can induce its own message (Van Obberghen-Schilling et al, 1988; Kim et al, 1990)

In contrast to contractile agonists, hyperplasia may occur on exposure of vascular smooth muscle cells to multiple growth factors, as occurs following deliberate vessel injury (Schwartz and Ross, 1984) or following sudden onset hypertension where there is evidence of endothelial injury or dysfunction (Owens and Reidy,1985), with possible generation of growth factors from several sources including endothelial cells, plasma, and smooth muscle cells themselves (Schwartz and Ross, 1984).

\section{Pressure- and flow-induced growth}

It has long been recognized that blood vessels themselves have the ability to sense changes in mechanical forces; flow/shear stress and stretch/wall stress. Both mechanical forces are capable to induce changes in vascular wall structure (Glagov et al, 1992; Folkow, 1982, 1990; Langille and O'Donnell, 1986; Langille et al, 1989). The site of mechanoreception in the vessel wall for transmural pressure is believed to be the vascular smooth muscle. On the other hand, shear stress is believed to be sensed by the endothelium. (Langille and O'Donnell, 1986; Hsieh et al, 1991). The cellular mechanisms by which structure is ultimately altered are unknown.

\section{Molecular mechanisms of action}

Vasoactive substances like angiotensin II, endothelin-1, catecholamines and arginine vasopressin bind to their receptor and initiate the intracellular signals via a G-protein, which in turn activates phospholipase C (fig. 1.5). Upon this activation two signalling pathways are operative: intracellular calcium mobilization and protein kinase $\mathrm{C}$ activation, which ultimately leads to contraction of vascular smooth muscle (Boyer et al, 1989; Simonson and Dunn, 1990; Grienling et al, 1989; Chardonnens et al, 1989; Raymond et al, 1990). The same messengers are involved in proto-oncogenes expression (Simonson and Dunn, 1990, Nakaki et al, 1990; Majesky et al, 1990; Berk and Alexander, 1989; Campbell-Boswell and Robertson, 1981).

The receptors for polypeptide growth factors are fundamentally different from other well characterized second messenger-associated hormone receptors in two important respects: growth receptors possess intrinsic tyrosine specific protein kinase activity and they share little or no amino acid sequence homology with the $G$ protein-linked receptors (Boyer et al, 1989; Yarden and Ullrich, 1988)(fig. 1.5). Growth factor receptor occupation stimulates the activity of the tyrosine kinase of the receptor, located in its intracellular 


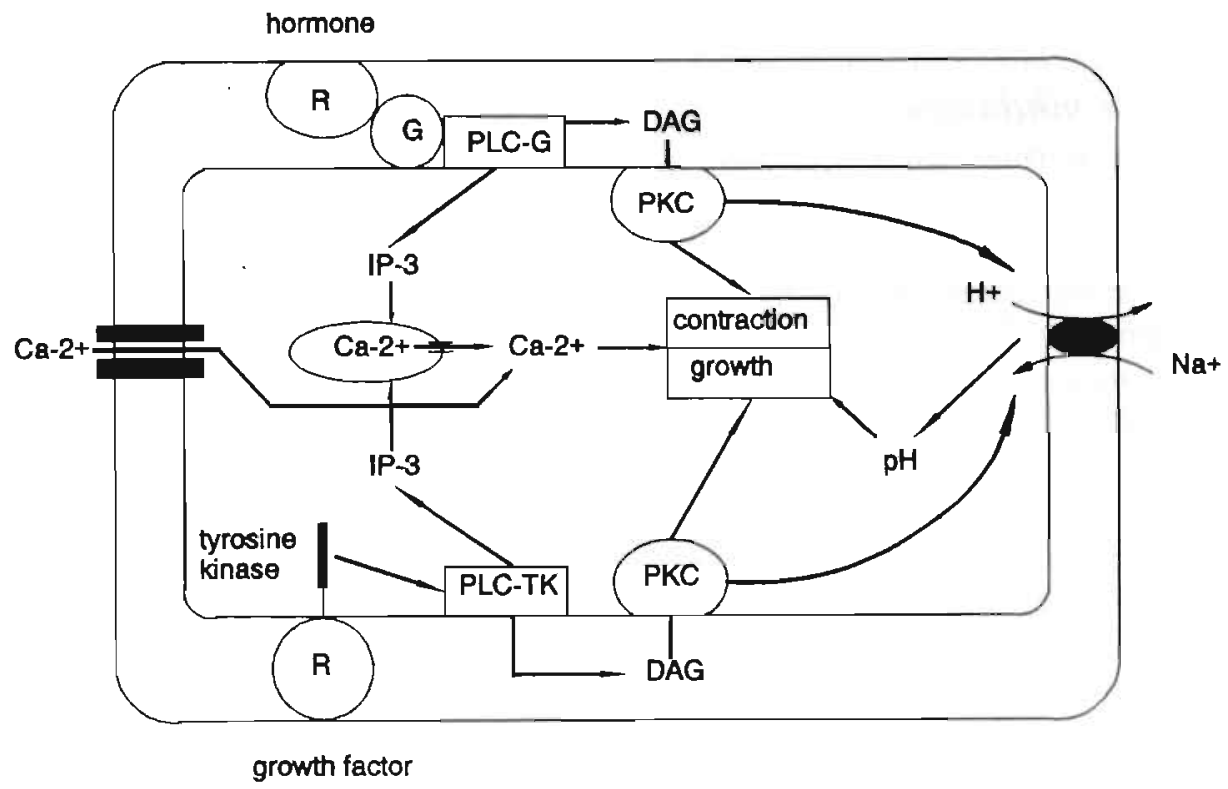

Figure 1.5 Schematic representation of the hormone-activated and growth factor-activated phospholipase $\mathrm{C}$ signalling pathway. $\mathrm{R}=$ receptor; $\mathrm{PLC}=$ phospholipase $\mathrm{C} ; \mathrm{PKC}=$ protein kinase $\mathrm{C} ; \mathrm{IP}_{3}=$ inositol-1,4,5-triphosphate.

domain. Tyrosine phosphorylation of a presumably different phospholipase $\mathrm{C}$, present in the cytosol or loosely associated with the plasma membrane, is linked with the phosphoinositide hydrolysis and diacylglycerol (DAG) formation. However, evidence now suggest that activation of growth factor receptors may not only result in tyrosine phosphorylation but also in activation of a poorly understood phosphatidylinositol kinase to form polyphosphoinositides different from those involved in the classical pathway (Coughlin et al, 1989). The physiological role of these lipids and their potential for generating novel intracellular mediators in the mitogenic response to growth factors remains speculative.

Exposure of growth-arrested vascular smooth muscle cells to serum and PDGF caused a decrease in $\mathrm{Na}^{+} / \mathrm{H}^{+}$exchange and an increase in intracellular $\mathrm{pH}$, whereas angiotensin II caused a decrease in intracellular pH (Berk et al, 1990; Rao et al, 1990). These findings suggest that hypertrophic and hyperplastic growth responses in vascular smooth muscle cells may be 
associated with differences in intracellular $\mathrm{pH}$ regulatory mechanisms. However, the precise role of the $\mathrm{Na}^{+} / \mathrm{H}^{+}$exchanger in regulating these $\mathrm{pH}$ changes remains to be defined.

\section{Growth inhibitors}

In normal adult vascular tissue, smooth muscle cells exhibit little evidence of proliferative activity in spite of their exposure to a host of stimulatory compounds. An explanation for this phenomenon lies in the presence of factors within the vessel wall that inhibit growth (Herman and Castellot, 1987; Scott-Burdon and Bühler, 1988; Castellot et al, 1987). One such component is heparin (Scott-Burden and Bühler, 1988; Castellot et al, 1987; Reilly et al, 1987; Resink et al, $1989^{\mathrm{a}}$ ), and although the inhibition of vascular smooth muscle cell growth by heparinoids has been well documented and the presence of heparin/heparan sulfate both in the basement membrane and interstitial matrix of vascular tissue is well established (Scott-Burden and Bühler, 1988; Castellot et al, 1987), the mechanism of action has not been fully elucidated. Inhibition of vascular smooth muscle cell growth by heparin has been correlated with the induction of synthesis of two matrix associated polypeptides (Scott-Burden and Bühler, 1988; Majack and Bornstein, 1984). Inhibition of growth by TGF- $\beta$ seems to be mediated by the same two polypeptides (Majack, 1987). In cultured vascular smooth muscle cells, EGF specifically reverses the antiproliferative effects of heparin. Conversely preincubation of cells with heparin leads to a reduction in their EGF binding capacity (Reilly et al, 1987; Resink et al, 1989). These fundings suggest a specific EGF-heparin growth-regulatory mechanism. In addition, also the basement membrane component laminin is suggested to be involved in vascular smooth muscle cell growth inhibition (Thyberg et al, 1990).

Another counter to the chronic stimulation of vascular smooth muscle cells to growth promotional compounds might be the constant exposure to nitric oxide (NO) (Grag and Hassid, 1989). The primary physiological effect of NO is to induce relaxation via elevation of intracellular cGMP levels within vascular smooth muscle cells (Rappoport and Murad, 1983). However, elevation of intracellular cGMP also leads to the depression of cAMP levels via activation of cGMP-dependent cAMP phosphodiesterase (Lungier and Schini, 1990) and this in turn may result in decreased stimulation of growth related metabolic events. The exact role of cAMP in proliferative metabolism has still not been clarified (Dumont et al, 1989).

\section{Current antihypertensive therapy and vascular structure.}

Irrespective of the underlying cause of elevated blood pressure, antihypertensive drugs lower blood pressure by an effect on one or more of the 
mechanisms involved in circulatory regulation. Due to the multiplicity of mechanisms involved in blood pressure control, antihypertensive properties are shared by the following classes of drugs:

- drugs reducing cardiac output by extracellular fluid volume depletion (diuretics) or by competitive inhibition of myocardial B-adrenoceptors (Bblockers);

agents which inhibit the activity of the renin-angiotensin system (ACEinhibitors, angiotensin II receptor antagonists);

drugs which produce arteriolar dilatation by a variety of mechanisms (ACE-inhibitors, $\alpha$-blockers, calcium channel blockers, hydralazine, minoxidil, nitrovasodilators);

sympatholytic agents, which may act centrally or peripherally to diminish sympathetic tone (methyldopa, clonidine, reserpine, ganglion-blockers, $\alpha$-blockers).

In this introduction I will further concentrate on the effects of antihypertensive treatment on structural changes that occur in hypertension.

Although large arteries contribute little to resistance, the development of aorta hypertrophy in rat models of hypertension has been widely studied. It is inhibited by drugs that block the effect of sympathetic nervous system (clonidine and methyldopa) (Carlier and Rorive, 1985) and by ACE inhibitors (Freslon and Giudicelli, 1983). By contrast, B-adrenoceptor antagonists, diuretics and hydralazine do not reverse hypertrophy of the aorta (Carlier and Rorive, 1985; Yamori et al, 1980). Conflicting evidence does not allow a definitive conclusion to be drawn regarding calcium antagonists. Clinical studies on hemodynamic properties of large arteries, show differential effects of antihypertensive drugs on large artery compliance and distensibility, measured by non-invasive Doppler methods. Hydralazine and related vasodilators as well as diuretics do not affect arterial compliance and distensibility. However, various ACE inhibitors and calcium antagonists increase arterial compliance. The $\beta$-adrenoceptor antagonists will either not affect or increase arterial compliance depending on the B-blocker used (Safar, 1988; Van Merode et al, 1989).

Studies evaluating the effect of antihypertensive treatment on resistancesized arteries have been performed in young as well as in adult SHR, to dissociate between primary and secondary adaptive changes in hypertension. SHR treated in utero with hydralazine and then up to the age of 20 weeks, had little effect on the structure (wall to lumen ratio, cross sectional diameter) of small arteries (pre-glomerular arteries)(Smeda et al, 1988 ). Long-term treatment of young and adult SHR with either B-blockers, a combination of hydralazine and guanethidine, or even a cocktail of hydralazine, reserpine and chlorothiazide had, in general, minimal effects on media thickness in 
resistance-sized arteries, despite a reduction of blood pressure to normal levels (Weiss, 1974; Weiss and Lundgren, 1978; Weiss et al, 1974; Warshaw et al, 1980). Young SHR that received a long-term treatment with hydralazine, or calcium antagonists showed no normalization of the wall-to-lumen ratio in mesenteric small arteries (Jespersen et al, 1985; Nyborg and Mulvany, 1985). Treatment of adult SHR with a combination of calcium antagonists and Bblockers, had little effect on vascular hypertrophy (Lundin and HallbackNordlander, 1984). On the other hand, several ACE inhibitors have consistently been found to induce regression of resistance-sized artery hypertrophy (Christensen et al, 1988; Christensen et al, 1989; Clozel et al, 1989; Black et al, 1989; Levy et al, 1988). ACE inhibitors also block the proliferative response of the arterial wall following mechanical injury or hyperlipidemia (Powell et al, 1989; Chobanian et al, 1990). Studies of isolated small arteries from patients with essential hypertension, show that although treatment causes a reduction in the wall-to-lumen ratio of subcutaneous small arteries, this regression is not complete (Heagerty et al, 1988; Aalkjaer et al, 1989).

Existing antihypertensive drugs are successful in reducing blood pressure, but less effective in reversing or preventing the development of abnormal artery structure. The fact that antihypertensive therapy is also less successful in reducing morbidity and mortality, implicates that the major cause for the cardiovascular complications in hypertension may be the structural alterations of the vascular tree rather than the elevated blood pressure per se (Lever, 1986). Understanding of the mechanisms of vascular growth control in general and vascular structural adaptions in the pathogenesis of hypertension and atherosclerosis may help in the selection and development of a new generation of drugs that could reduce the incidence of hypertension-induced cardiovascular diseases.

\section{THE ENDOTHELIUM}

\section{Endothelium and circulation}

The endothelium, which forms the innermost layer of blood vessels, has been recognized to play an important role in the circulation. Endothelial cells are not only involved in capillary transport and exchanges between blood and tissue, but also in maintaining the fluidity of the blood. Many factors synthesized in endothelial cells can keep the surface non-adhesive and nonthrombogenic for circulating cells (Gryglewski et al, 1988).

The endothelium can modify the tone of the underlying vascular smooth muscle by activation and inactivation of circulating hormones and other plasma constituents, and synthesis and secretion of vasodilator and vasoconstrictor 
substances (Furchgott and Vanhoutte, 1989; Lüscher, 1988). In addition, endothelial cells may modulate vascular structure by regulating the supply of blood born mitogens, and the synthesis, storage, and release of vascular smooth muscle mitogens (Gajdusek et al, 1980; Castellot et al, 1981; Mantovani and Dejana,1989; Davies,1986).

\section{Endothelial effects on vascular tone}

Endothelium-derived vasoactive substances can be synthesized and released by circulating and local hormones, physical and chemical factors, and factors involved in hemostasis. The resulting endothelium-dependent effect on vascular tone depends on the anatomical location of the blood vessels and the stimuli involved, and whereby species differences have been noted.

Endothelium-derived relaxing factor (EDRF)(Furchgott, 1984) is a labile substance which accounts for the vascular relaxation induced by acetylcholine and other endothelium dependent vasodilators. It also inhibits platelet aggregation and adhesion. All these actions are mediated by stimulation of soluble guanylate cyclase (Azuma et al, 1986; Radomski et al, 1987 ${ }^{\mathrm{a}}$; Moncada et al, 1988). The chemical nature of EDRF has been identified as nitric oxide (NO)(Moncada et al, 1988), which is synthesized by a constitutive nitric oxide synthase from L-arginine (Moncada and Higgs, 1991). NO is released in response to shear stress, pressure, platelet-derived products (adenosine diphosphate, thrombin, serotonin) and certain hormones (bradykinin, histamine, noradrenaiin, substance $P$ and vasopressin)(Rubanyi et al,1986; Pohl et al,1986; De Mey et al, 1982; Housten et al, 1985; Cocks et al, 1985; Van de Voorde and Leusen, 1983; Cocks and Angus, 1983; Katusic et al, 1984). Evidence from the use of L-arginine analogues, $\mathrm{N}^{\mathrm{G}}$-monomethyl-L-arginine ( $\mathrm{L}$ NMMA), $\mathrm{N}^{\mathrm{G}}$-nitro-L-arginine (L-NNA), its methyl ester L-NAME, and Niminoethyl-L-ornithine (L-NIO), which are all inhibitors of NO generation in vascular tissue, supports a basal secretion of NO in vivo (Moncada and Higgs, 1991).

In addition to NO, platelet-derived products, thrombin, histamine, angiotensin II, shear stress, and hypoxia can also stimulate the endothelia] production of prostacyclin $\left(\mathrm{PGI}_{2}\right)$ (Baenziger et al,1981; Rubanyi et al, 1986; Lüscher et al, 1986; Toda, 1984; Francos et al,1985; Busse et al, 1984; Moncada and Vane, 1979). Most commonly, prostacyclin causes relaxation of isolated vascular rings or strips of most arteries. Vascular relaxations induced by prostacyclin is mediated by cyclic AMP (Furchgott and Vanhoutte, 1989; Ito et al, 1980). In analogy to $\mathrm{NO}, \mathrm{PGI}_{2}$ possesses potent inhibitory effects on platelet adhesion and aggregation, and there is also a basal release of $\mathrm{PGI}_{2}$ in vivo (Radomski et al, $1987^{\mathrm{b}}$ ).

It is likely that besides $\mathrm{NO}$ and $\mathrm{PGI}_{2}$ the endothelium releases an 
endothelium-derived hyperpolarizing factor (EDHF) (Vanhoutte, 1987). In the canine femoral artery, endothelium-dependent relaxations to acetylcholine are reduced after inhibition of sodium-potassium ATPase by ouabain (De Mey and Vanhoutte, 1981 $)$. Acetylcholine causes endothelium-dependent increases in membrane potential of vascular smooth muscle cells that can be blocked by blocked by ouabain (Feleton and Vanhoutte, 1988). NO does not mimic the response, indicating that another substance must be involved.

Although most mammalian species show endothelium-dependent relaxations, some species differences are observed (Kalsner, 1985; Shimokawa et al, 1987). It appears that within the cardiovascular system endotheliumdependent relaxations to acetylcholine become more prominent as the arterial diameter decreases (De Mey and Gray, 1985; Owens and Bevan, 1985; Aalkjaer et al, $1987^{b}$ ). As a rule, endothelium-dependent relaxations are more pronounced in arteries than in veins (De Mey and Vanhoutte, 1982). Even within the same species, a considerable heterogeneity of endothelium-dependent responses among different vascular beds has been observed (Katusic et al, 1984; Lüscher, 1988).

While investigating the response of blood vessels to endotheliumdependent relaxing factors, it became apparent that in several blood vessels, certain of these agents caused endothelium-dependent contractions rather than relaxations (De Mey and Vanhoutte, 1982). Endothelium-dependent contractions can be elicited by chemical and physical forces, precursor substances of cyclooxygenase products and receptor-operated stimuli (Lüscher et al, 1992).

In isolated canine femoral, coronary, and cerebral arteries hypoxia and anoxia augment contractile responses; this response to anoxia is markedly reduced after removal of the endothelium (De Mey and Vanhoutte, 1982 1983; Katusic et al, 1986; Rubanyi and Vanhoutte, 1985). The anoxic endothelium-dependent contractions are not blocked by inhibitors of phospholipase $A_{2}$ or lipooxygenase, which rules out a product of the metabolism of arachidonic acid as the mediator. In cerebral arteries of the cat, increase in transmural pressure cause vascular smooth muscle cell depolarization and vasoconstriction (Harder et al, 1989; Harder, 1984). This pressure-induced vasoconstriction is endothelium-dependent and its nature remains to be determined. Mechanical stretch can evoke endothelium-dependent contractions in isolated canine basilar artery (Katusic et al, 1987) and rat aorta (Rinaldi and Bohr, 1989). The endothelium-dependent contractions to stretch involve the metabolism of arachidonic acid. Cultured endothelial cells of various species including man produce and release the potent vasoconstrictor peptide endothelin (Yanagisawa et al, 1988; Yanagisawa and Masaki, 1989). The expression of preproendothelin mRNA is stimulated by vasopressor hormones such as adrenaline, Ang II, vasopressin, substances derived from aggregating platelets (TGF-B), coagulation products (thrombin), and cytokines (interleukin- 
1)(Yanagisawa et al, 1988; Yanagisawa and Masaki, 1989; Yoshizumi et al, 1990). In addition, the production of endothelin is regulated by inhibitory stimuli. In particular, NO and $\mathrm{PGI}_{2}$ reduce ET-1 production (Boulanger and Lüscher, 1990; Saijonmaa et al, 1990); circulating hormones such as atrial natriuretic factor also reduce the formation of the peptide (Saijonmaa et al, 1990).

Endothelial cells are the major site of conversion of Ang I to Ang II in the circulation ( $\mathrm{Ng}$ and Vane, 1967,; Saye et al, 1984). Moreover, cultured bovine endothelial cells contain a prorenin-activating enzyme, renin-like activity, and immunoreactive angiotensinogen, Ang I, II, and III (Dzau, 1986; Kifor and Dzau, 1987; Lilly et al, 1985). Anatomically the vascular wall RAS seems to be mainly located in the intimal and adventitial region of the vascular wall. Therefore, the vascular RAS may function as an EDCF by providing Ang II acting directly on vascular smooth muscle as well as on prejunctional receptors of adrenergic nerve endings (Antonaccio and Kerwin, 1981; Dzau, 1986).

The endothelium may protect the vascular smooth muscle from vasoconstrictor hormones by acting as physical barrier and through the basal and stimulated release of endothelium-derived relaxing factors. How the balance between all these interacting vasoactive substances is maintained under physiological conditions and altered under various pathological conditions is currently subject of intense research.

\section{Endothelial effects on vascular structure}

Besides the important contribution of the endothelium in regulating vascular tone, indirect evidence suggests that the endothelium may also be involved in modulating vascular structure. As already discussed, it is known that circulating and endothelium-derived vasoactive substances are mitogenic for vascular smooth muscle cells, such as catecholamines, serotonin, endothelin and angiotensin II. On the other hand, endothelium-derived vasodilators, like nitric oxide and $\mathrm{PGI}_{2}$, inhibit the proliferation of isolated vascular smooth muscle cells.

Endothelial cells can also produce polypeptide vascular smooth muscle cell growth affecting substances as shown in cell culture experiments (JosephSilverstein and Rifkin, 1987; Davies, 1986; DiCorletto et al, 1983; Castellot et al, 1981; Koo and Gotlieb, 1989; Mantovani and Dajena, 1989). In analogy to endothelium-derived vasoconstrictor and vasodilator substances, the endothelium forms inhibitors (TGF- $\beta$ and interferon-gamma)(Joseph-Silverstein and Rifkin, 1987; Hannan et al, 1988; Pober et al,1986) and promoters (EGF, FGF, PDGF, IL-1, IL-6, thrombospondin)(Fox and DiCorletto, 1986; Harlan et al, 1986; Gajdusek et al, 1986; Hannan et al, 1988; Vlodavsky et al, 1987; 
Warner et al, 1987 ; Mantovani and Dajena, 1989; Vischer et al, 1985) of vascular smooth muscle cell growth.

Cultured endothelial cells themselves are not mitogenically sensitive to PDGF, but expression levels for both the A and B chain can be stimulated by a number of other growth factors (Dicorletto and Fox, 1988). In addition, IL-1 and TGF- $B$ have been reported to stimulate the production of ET-1 mRNA and protein by cultured endothelial cells (Yoshizumi et al, 1990; Kurihara et al, 1989). Il-1 can even induce IL-1 and PDGF production in human endothelial cells (Warner et al, 1987 ; Mantovani and Dejana, 1989). On stimulation with PDGF or TGF- $\beta$, endothelial cells release thrombospondin which is an extracellular matrix glycopeptide with vascular smooth muscle cell mitogenic activity (Majack et al, 1985; Scott-Burden and Bühler, 1988; Penttinen et al, 1988). In combination with EGF, it stimulates mitogenesis to a similar extent as that induced by PDGF (Majack et al, 1986).

The production of PDGF by endothelial cells in vivo is minimal but this may be enhanced by their exposure to components of the coagulation (Harlan et al, 1986; Gajdusek et al, 1986). Injury to the endothelium stimulates the secretion of bFGF by endothelial cells, while release of FGF molecules from blood born cells following endothelial denudation may give rise to an accumulation of growth factor within the basement membrane and/or the extracellular matrix (Gajdusek and Caron, 1989). This could generate a slow release of growth factor and vascular smooth muscle cell proliferation long after the repair of the endothelium.

As in the control of vascular tone there is probably a complex interplay between blood, blood cell, endothelium, extracellular matrix and even vascular smooth muscle derived growth affecting substances in maintaining vascular structure. How this endocrine, paracrine and autocrine communication is regulated remains unknown.

\section{Changes in hypertension}

In rat models of hypertension, endothelial cells exhibit distinct morphological changes (Gabbiani et al, 1979; Hüttner and Gabbiani, 1983; Limas et al, 1982; McGuire and Twietmeyer, 1985). While the integrity of the endothelium is preserved, an increased permeability, number, volume and replication rate of endothelial cells occur. Hypertension is also associated with an increased adherence of circulating blood cells to the endothelial layer (Limas et al, 1982; Chobanian et al, 1986). The adhering cells are mainly granulocytes, monocytes and lymphocytes. The number of endothelial cytoplasmatic organelles are increased in hypertension (Hüttner and Gabbiàni, 1983). All these morphological changes of the endothelium might be associated the functional changes of the vascular endothelium in hypertension. 
Endothelium-dependent relaxations to acetylcholine, ADP, and thrombin are impaired in aortic rings of rats with spontaneous, salt-induced deoxycorticosterone acetate (DOCA-salt) and renal hypertension, and in aortic. coarctation (Lüscher, 1988; Lockette et al, 1986; Winquist et al, 1984; Konishi and Su, 1983; Van de Voorde and Leusen, 1984). Similar changes occur in carotid artery, in mesenteric and cerebral resistance arteries of rats with spontaneously hypertension (De Mey and Gray, 1985; Lüscher et al, 1988). A decrease in endothelium-dependent relaxation in hypertension could be due to a decreased production of or decreased smooth muscle cell sensitivity to $\mathrm{EDRF}$, an impaired diffusion of the substance or due to the release of an endothelium-derived contracting factor (Lüscher, 1988). In salt-induced hypertension, the reduced endothelium-dependent relaxations are due to the combination of a diminished release of EDRF and a lesser sensitivity of the vascular smooth muscle to the relaxing effect of the factor (Lüscher et al, 1987). In the spontaneously hypertensive rat, the reduction in endotheliumdependent relaxation can not be due to a reduced release of EDRF but to the liberation of a constricting factor by the endothelial cells (Lüscher and. Vanhoutte, 1986). The release of the factor involved requires the activation of cyclo-oxygenase.

Thus, in hypertension and in other cardiovascular diseases (Vanhoutte, 1988), the ability of the endothelium to release EDRF's is blunted, while, if anything, its ability to release EDCF's is augmented. It is tempting to assume that this imbalance may contribute to the increase in peripheral resistance characteristics of the disease (Lüscher, 1990).

In analogy to an imbalance of endothelium-derived relaxing and contracting factors in hypertension, the same could hold for vascular smooth muscle growth control in the vascular wall. There is now some evidence to suggest that phenotypic conversion of vascular smooth muscle can occur in the absence of damage to the endothelium. This suggests that there might be an imbalance between growth inhibiting and growth stimulating substances within the endothelium, possibly involving endothelial substances and growth factors.

\section{GOAL OF THE PRESENT STUDY}

Hypertension and atherosclerosis are characterized by structural changes of the vascular wall. In hypertension different arterial smooth muscle cell growth responses are observed depending on the anatomical location. Media hypertrophy of large arteries is due to arterial smooth muscle cell hypertrophy/hyperploidy. In small resistance-sized arteries it has been attributed to arterial smooth muscle cell hyperplasia. The reasons for this regional heterogeneity are not clear. Growth responses of arterial smooth muscle cells may be influenced 
by several mechanisms. Phenomena such as hemodynamics, externally supplied trophic stimuli and growth inhibitory influences and nervous input could play a role. Also mechanisms that are endogenous to the vascular wall could be involved. Arterial smooth muscle cells and endothelial cells produce factors that affect growth responses in an autocrine or paracrine manner. In this respect also the extracellular matrix should be mentioned.

We therefore started to evaluate the effects of long-term exposure to serum growth factors of isolated renal arteries from adult normotensive rats on changes in DNA synthesis, structure and mechanical activity (chapter 3 ). This is done in the absence of the endothelium and sympathetic nerve endings, to rule out their influence on vascular growth responses.

To evaluate whether intravascular phenomena contribute to local differences in growth responses of the arterial wall, the extent of DNA synthesis and structural changes to organoid culture are studied in a broad variety of arterial preparations (chapter 4). The influence of the endothelium on these mitogenic responses of arterial smooth muscle of different anatomical origin are also described.

The observations from the previous experiments suggest a role for the endothelium in modulating vascular smooth muscle growth responses in vitro. To evaluate whether this endothelial modulation is altered in hypertension, DNA and protein synthesis are compared in isolated renal and carotid arteries of normotensive and hypertensive rats. In order to dissociate primary causal changes from secondary adaptive changes to elevated blood pressure, the experiments are performed in young and adult rats, in adult normotensive rats made hypertensive by aorta coarctation and mouse Ren- 2 gene hypertensive rats (chapter 5).

Because arterial smooth muscle cells and endothelial cells can synthesize growth factors that influence vascular smooth muscle cell growth, chronic effects candidate autocrine and paracrine factors on DNA synthesis are elucidated in carotid and renal arteries isolated from adult normotensive rats (chapter 6). As nitric oxide has been suggested to be involved in inhibitory influences of vascular smooth muscle growth, the involvement of this labile endothelium-derived relaxing factor and its generating enzymes are discussed.

To compare the in vitro observations in isolated arteries of the rat with an in vivo situation, DNA synthesis and structural changes are examined in different arteries of young normotensive rats and hypertensive rats (chapter 7). Because the sympathetic nervous system and the renin-angiotensin system are suggested to be involved in the development of hypertension, young normotensive rats were infused with angiotensin II or phenylephrine (chapter 8). Also in these experiments a broad variety of arterial preparations were evaluated. The results are related to in vivo DNA synthesis in age matched genetic hypertensive rats. 


\section{INTRODUCTION}

In vivo, angiotensin II induces an acute and slow pressor action. This slow pressor action is believed to be a consequence of arterial structural changes. In chapter 9 we evaluated whether angiotensin II elicits besides its fast transient contraction also a low chronic increase of tone in isolated arteries. For this purpose we studied isometric force development and structural parameters in the presence of angiotensin II.

Finally, the observations obtained in this study are summarized and discussed (chapter 11). 



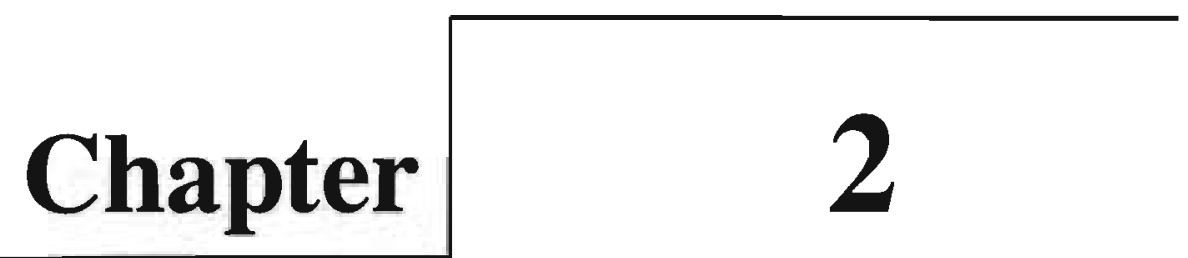

Materials and Methods 


\section{EXPERIMENTAL APPROACHES}

\section{Animals}

Most experiments described in this thesis were performed with WKY and SHR that were derived from inbred strains of the Central Animal Facilities of the University of Limburg (Maastricht, The Netherlands). The spontaneously hypertensive rat (SHR), originally derived from normotensive Wistar-Kyoto (WKY) rats, is the most commonly used model of genetic hypertension. To dissociate primary interstrain differences from secondary adaptive changes to elevated blood pressure with respect to arterial smooth muscle growth, experiments were performed in 6 and 20 week old animals. At the age of 6 weeks no differences in blood pressure could be observed between WKY and SHR. To investigate whether interstrain differences could exist between normotensive animals, experiments were included with 20 week old normotensive Wistar (WIS) rats (Charles River, Germany).

In order to obtain supplementary information about secondary adaptive changes to high blood pressure, adult WKY were made hypertensive by partial aorta coarctation. For this purpose the abdomen of 17 week old WKY was opened under ether anesthesia and the abdominal aorta was tied between the superior mesenteric artery and the left renal artery onto the shaft of a stainless steel rod (diameter $0.7 \mathrm{~mm}$ ). This tool was subsequently removed, leaving the abdominal aorta partially obstructed. The abdomen was closed and the animals were allowed to recover. Three weeks later ( 20 weeks of age) these hypertensive animals were prepared for experimentation.

Recently the mouse Ren-2 gene was introduced into the genome of Sprague-Dawley (SDR) rats, producing hypertensive animals (Mullins et al, 1990). With this model of experimental hypertension, experiments were performed in 11 week old transgenic animals (TGR) and age matched SDR. The animals were derived from the AFRC Centre for Genome Research, University of Edinburgh (Edinburgh, UK).

All animals were maintained on a $12 / 12 \mathrm{hr}$ dark light cycle, were fed with a standard food (Hope Farms, The Netherlands) and had free access to water.

\section{Arteries}

To study regional heterogeneity of vascular smooth muscle growth responses (Schwartz et al, 1986; Owens, 1989; Mulvany et al, 1985; Owens et $\mathrm{al}, 1988^{\mathrm{a}}$ ), experiments were performed in isolated elastic, muscular and resistance-sized arteries. These different types of vessels included thoracic aorta 
$(A O)$, carotid artery (CA), superior mesenteric artery (MA), renal artery (RA), femoral artery (FA), and mesenteric (MrA) and intrarenal (RrA) resistancesized artery. To evaluate whether regional differences in paracrine and autocrine regulation of vascular smooth muscle growth could play a role, experiments were performed in the presence and absence of endothelium.

\section{Experimental design}

The classical experimental approaches to evaluate arterial smooth muscle growth control are arterial injury in situ (Schwartz et al,1986; Jackson and Schwartz, 1992) and enzymatically dispersed smooth muscle cells in culture (Chamley-Campbell et al, 1979). In the arterial injury model it is difficult to discriminate an influence of potential autocrine and paracrine growth factors from the effects of media injury and of plasma- and platelet-derived mitogens. On the other hand, extrapolation of observations done in cell culture experiments to the intact vasculature is difficult for several reasons. First, endothelial cells and arterial smooth muscle cells are obtained from a limited number of sources. Both cell types, however, display functional properties that vary locally (Vanhoutte, 1978; De Mey and Vanhoutte, 1981 a , 1982; Furchgott and Vanhoutte, 1989; Heesen and De Mey, 1990), which may also be the case for the responsiveness to mitogens and growth inhibitors. Furthermore, isolated vascular smooth-muscle cells are selected growth-prone cells, which already include an alteration in growth control (Chamley-Campbell et al, 1979; Schwartz et al, 1986). Second, cell culture experiments give poor information about the ultimate structural consequence of a given growth affecting condition in a tissue.

In this thesis we used arterial organ culture as an intermediate type of model to the above mentioned approaches, to evaluate growth responses in the isolated arterial wall (fig. 2.1). The advantage of this model is primarily the possibility to evaluate structural changes in the "intact" vascular wall, i.e vascular smooth muscle cells at their normal density and natural microenvironment, under well-defined culture conditions and to correlate structural changes with changes in contractility. This type of approach is also suited for every type of blood vessel that can be isolated, including resistance-sized arteries that are involved in the regulation of vascular resistance and local blood flow in vivo. Selective removal of arterial components of the vascular wall, such as the endothelium and nerve endings, make it possible to study paracrine and autocrine mechanisms that are involved in arterial smooth muscle growth control.

An important issue in culture experiments is the composition of the medium used. Culture of vascular smooth muscle cells in vitro has led to important findings in this regard (Chamley-Campbell et al, 1979). Serum, an 


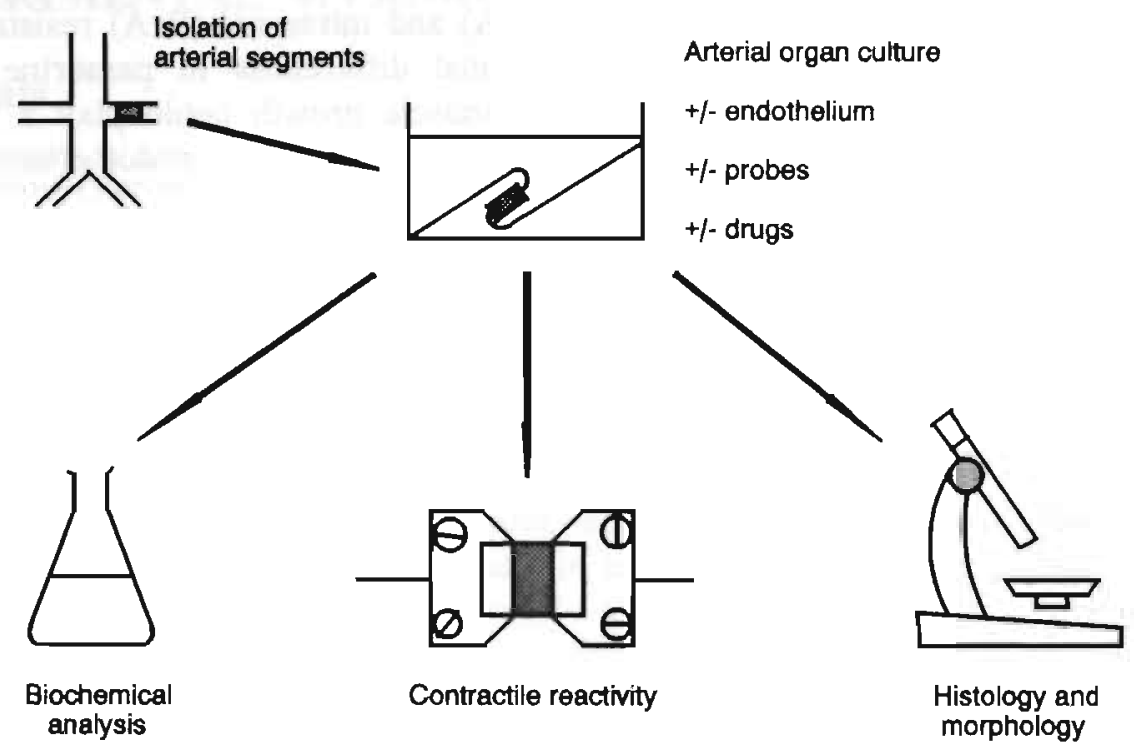

Figure 2.1 Methods used for the evaluation of long-term effect of various agents on isolated arteries.

unphysiological stimulus for vascular smooth muscle cells, causes them to proliferate as they do in injured or diseased vessels (Ross, 1981). In previous studies we showed that fetal calf serum (FCS) gave a maximal growth response, as measured by medial DNA synthesis, in isolated renal arteries that were denuded of endothelium (De Mey et al, 1989, 1991 ${ }^{\mathrm{a}}$ ). However, the problem encountered with the use of serum is that this fluid is a complex and undefined mixture that contains unknown concentrations of various nutrients, hormones, binding proteins, and other compounds. This lack of chemical definition prevents precise study of the effect of vascular smooth muscle growth affecting substances.

To maintain vascular smooth muscle cells in a quiescent, viable state, plasma-derived serum (PDS), PDS stripped from progression factors (CMSPDS) or defined serum-free media were used (Vogel et al, 1978; Libby and O'Brien, 1983). Incubation of arterial smooth muscle cells with these sera, made it possible to study effects of individual growth affecting factors on arterial smooth muscle cell growth control in vitro.

To evaluate the distribution of arterial structural responses in the vascular 
tree after induction of hypertension, experiments were performed in young WKY and SHR with marginally elevated blood pressure. To mimic hyperactivity of the sympathetic nervous system and the renin-angiotensin-aldosterone system, hypertension was induced in young WKY by continuous infusion of phenylephrine or Ang II. Both systems are supposed to be involved in "slowpressor" mechanisms in hypertension (Lever, 1986).

To evaluate whether the suggested "slow-pressor" effect of Ang II in vivo (Lever, 1986) is based on structural and/or functional alteration of the arterial wall, tonic tone of isolated arterial segments was studied during continuous exposure to Ang II in vitro. These experiments needed a combination of continuous recording of isometric force development, as measured with a Mulvany-Halpern type of myograph, and arterial organ culture.

\section{TECHNIQUES}

\section{Arterial organ culture}

All experiments were performed on artery segments that were isolated under sterile conditions from rats that were killed by a sharp blow on the back of the head and exsanguination. The segments that had been collected in Hanks' balanced salt solution (HBSS; GIBCO, Paisly, UK) were chemically sympathectomized by incubation at $37^{\circ} \mathrm{C}$ for 10 minutes in sterile bicarbonate free Krebs-Ringer solution ( $\mathrm{pH}=4.00$ ), containing $300 \mu \mathrm{g} / \mathrm{ml}$ 6-hydroxydopamine (Sigma Chemicals St.Louis, Mo, USA)(Aprigliano and Hermsmeyer, 1976) and rinsed twice in $5 \mathrm{ml}$ HBSS supplemented with $1200 \mathrm{IU} / \mathrm{ml}$ penicillin (Gist-Brocades, Delft, The Netherlands) and $1200 \mu \mathrm{g} / \mathrm{ml}$ streptomycin (Pharmachemie, Haarlem, The Netherlands) for 10 minutes.

To remove the endothelium, the arterial segments were transferred in 10 $\mathrm{ml}$ HBSS solution, containing $100 \mathrm{IU} / \mathrm{ml}$ penicillin and $100 \mu \mathrm{g} / \mathrm{ml}$ streptomycin, and the shaft of a hypodermic needle was passed through the lumen of the vessel. The diameter of this tool differed for the different types of vessel that were used, thereby taking care not to distend or damage the media of the preparation (De Mey et al, 1989, 1991 ${ }^{\mathrm{a}}$ ). The success of this method of gentle mechanical denudation was confirmed by the absence of endothelium-dependent relaxing responses to acetylcholine and histamine and endothelium-dependent immunoreactivity for von Willebrand factor and angiotensin converting enzyme (ACE). Whether the denudation was accompanied by media injury was investigated by comparing the maximal contractile response to $125 \mathrm{mM}$ potassium (section: sera and physiological salt solutions) in denuded and intact arteries, using an isometric myograph (section: contractile reactivity).

The organ culture methods that were used for isolated artery segments 
were based on culture methods described for vascular smooth muscle cells, strips and rings of arteries (Chamley-Campbell et al, 1979; Mauger et al, 1975; Hume, 1980; Merrilees and Scott, 1982). Artery segments were mounted on the shaft of a hypodermic needle with an appropriate diameter or a sterile wire with a diameter of $0.25 \mathrm{~mm}$ (Dentaurum, Pfortzheim, Germany) and individually suspended in Dulbecco's minimal Eagle's modified medium (Gibco), containing $2 \mathrm{mM}$ glutamine (Gibco), $100 \mathrm{IU} / \mathrm{ml}$ penicillin, and 100 $\mu \mathrm{g} / \mathrm{ml}$ streptomycin. This basal culture medium was supplemented with different sera (20\% dFCS, 5\% WBS, 5\% PDS, or 5\% CMS-PDS), in the absence or presence of mediators, probes and/or drugs (fig. 2.1). Under these conditions the arteries were maintained for 3,4 or 14 days in culture at $37^{\circ} \mathrm{C}$ in an atmosphere of $95 \%$ air and $5 \% \mathrm{CO}_{2}$ at $97 \%$ humidity. The arterial preparations were regularly transferred to culture dishes filled with fresh medium.

At the end of organ culture the arterial segments were rinsed twice in 5 $\mathrm{ml}$ oxygenated Krebs-Ringer bicarbonate solution (KRB, section: sera and physiological salt solutions) for 15 minutes. The preparations were either directly fixed, mounted in an isometric myograph for recording of mechanical activity or prepared for biochemical analysis. The former arterial segments were fixed for $30 \mathrm{~min}$ in periodate-lysine-paraformaldehyde (Mclean and Nakoine, 1974) or phosphate buffered formaldehyde ( $4 \% \mathrm{v} / \mathrm{v}, \mathrm{pH}=7.4)$ at $37^{\circ} \mathrm{C}$, and stored at $4^{\circ} \mathrm{C}$ in phosphate buffered saline or ethanol $(70 \% \mathrm{v} / \mathrm{v})$ until they were processed for histological examination.

\section{Contractile reactivity}

To study mechanical and pharmacological properties of freshly isolated and tissue cultured arterial segments, ring preparations were mounted in a myograph for recording of isometric tension development (Mulvany and Halpern, 1977)(fig. 2.2). The organ chamber was filled with KRB that was maintained at $37^{\circ} \mathrm{C}$ and aerated with $95 \% \mathrm{O}_{2}-5 \% \mathrm{CO}_{2}$. Two stainless steel wires (diameter $0.04 \mathrm{~mm}$ ) were inserted in the lumen of the arterial preparations and were connected to a displacement device and an isometric force transducer (Kistler Morse DSC6 or Statham UC3). All arterial preparations were stretched to the diameter at which their individual mechanical performance was maximal. To achieve this, the arterial diameter was stepwise increased, and the preparations were intermittently exposed to $125 \mathrm{mM}$ potassium (K-KRB) until maximal contractile responses were obtained.

Contractile responses were expressed as increase in wall tension (increase in force/twice the segment length; $\mathrm{mN} / \mathrm{mm}$ ), increases in wall stress (increase in wall tension/mean media thickness; $\mathrm{mN} / \mathrm{mm}^{2}$ ) and/or relative to the maximal response to $125 \mathrm{mM}$ potassium. 


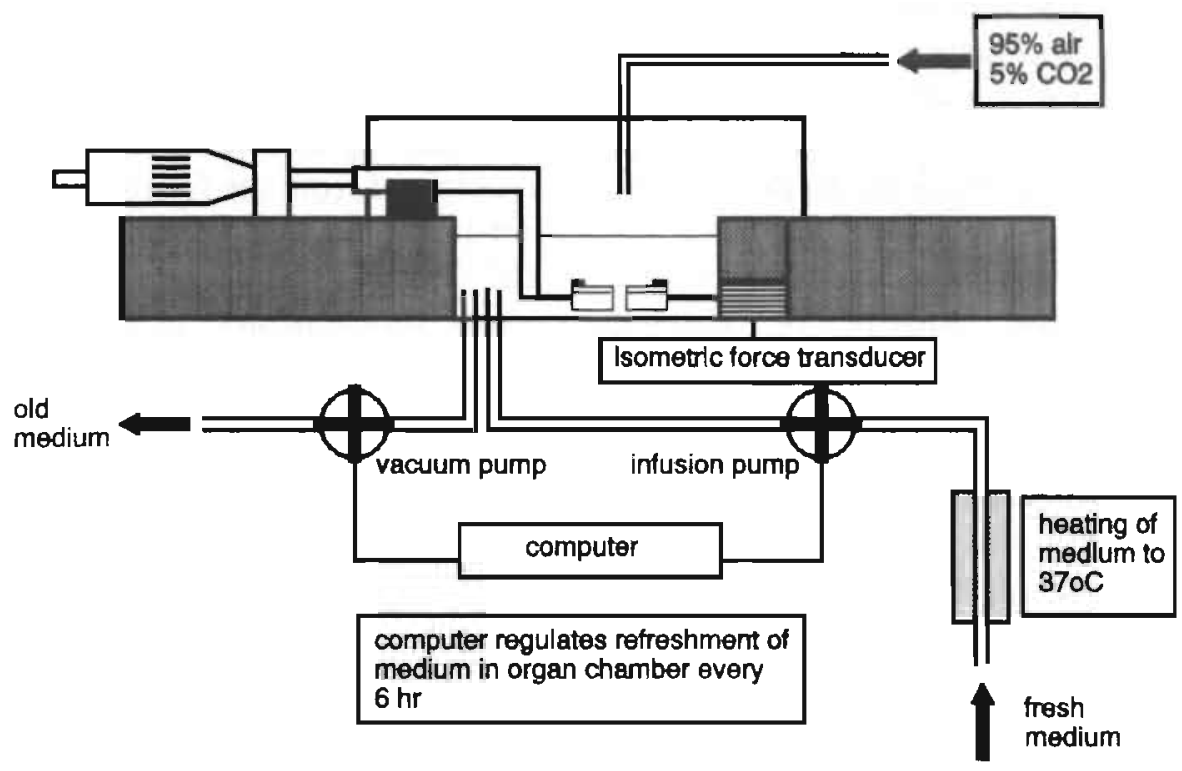

Figure 2.2 Myograph equipment for continuous recording of mechanical activity during organ culture of isolated arteries.

The recordings of contractile reactivity were terminated by exposing resting arterial preparations at optimal lumen diameter and at $37^{\circ} \mathrm{C}$ to periodate-lysine-paraformaldehyde or phosphate buffered formalin $(\mathrm{pH}=7.4)$. Thirty minutes later the fixed arterial segments were removed from the recording apparatus and stored at $4^{\circ} \mathrm{C}$ in phosphate buffered saline or ethanol $(70 \% \mathrm{v} / \mathrm{v})$.

\section{Structural changes}

Fixated arterial segments were embedded in paraffin (Paraplast $^{+}$, Sherwood, St.Louis, USA) and cross sections of $4 \mu \mathrm{m}$ (microtome type 820 , American Optical, USA) were stained with hematoxylin and eosin for evaluation of overall structure.

Quantitative morphology was performed on cross sections after staining with Lawson's solution, which highlights the elastic laminae, using video images generated by a Zeiss Axioscope (Zeiss, Germany), a standard CCD camera (Stemmer, Germany) and commercial software (JAVA; Jandel 
Scientific Video Analysis Software, Jandel Scientific, Corte Madera, CA, USA). The internal circumference (IC), i.e. the length of the internal elastic lamina, was determined along with the cross sectional area of the tunica media (CSA), i.e. the area enclosed between the internal and external elastic lamina. From IC the lumen radius ( $\mathrm{r}$ ) was calculated assuming a circular configuration $(\mathrm{r}=\mathrm{IC} / 2 \pi)$. Mean media thickness $(\mathrm{T})$ was obtained from the formula CSA $=$ $\pi(r+T)^{2}-\pi r^{2} . M t / r$ was taken as an estimate of the wall to lumen ratio.

To estimate DNA synthesis, either the thymidine analogue 5-bromo-2'deoxyuridine (BrdUrd) or ${ }^{3} \mathrm{H}$-thymidine was supplemented to the culture medium. Protein synthesis was determined by ${ }^{14} \mathrm{C}$-leucine incorporation. The precursors were either present continuously during the whole culture experiment or $24 \mathrm{hr}$ prior to fixation of the arterial segments.

For preparations that had been cultured in the presence of BrdUrd, an indirect enzyme-labeled antibody technique (monoclonal mouse-anti-BrdUrd) with peroxidase conjungated second step reagents (peroxydase conjungated rabbit-anti-mouse) was used to visualize nuclei that had incorporated the thymidine analogue (Gratzner, 1982). To quantify the incorporation of BrdUrd, the percentage of nuclear profiles that stained for peroxidase was determined for the media and/or adventitia.

For the evaluation of DNA- and protein synthesis during tissue culture in preparations exposed to radioactive labeled precursors, arterial preparations were washed twice in Krebs-Ringer solution, dried between tissue paper and solubilized over night in $100 \mu \mathrm{l} 1 \mathrm{~N} \mathrm{NaOH}$ at room temperature. Of this solution $80 \mu \mathrm{l}$ was transferred in $5 \mathrm{ml}$ scintillation liquid and counted, to determine the amount of incorporated thymidine and leucine. DNA and protein synthesis were expressed relative to the DNA content, measured by a fluorometric assay (Labarca and Paigen, 1980). In the remaining $20 \mu \mathrm{l}$ of the tissue extract, $10 \mu \mathrm{l}$ was neutralized in $1 \mathrm{ml}$ phosphate buffer and added to 1 $\mathrm{ml}$ of Hoechst solution $(93.6 \mu \mathrm{l} / \mathrm{l}$ bisbenzimidazole). After mixing the DNA content was measured photometrically (excitation wave length $360 \mathrm{~nm}$, emission wave length $460 \mathrm{~nm}$; AMINCO spectrophotometer, SPF-500C). Calf thymus DNA was used as standard.

The recent development of monoclonal antibodies to proliferationassociated antigens offered another approach to quantify DNA synthesis. Proliferating cell nuclear antigen (PCNA) is a nuclear antigen which is essential for DNA synthesis (Bravo et al, 1987; Lee and Hurwitz, 1990). To estimate DNA synthesis by PCNA immunohistochemistry, paraffin slides (4 m) were successively incubated with mouse monoclonal anti-PCNA antibody PC 10 (DAKO, Glostrup, DK), biotinylated rabbit anti-mouse immunoglobulin, and avidin and biotinylated horseradish peroxidase. Diaminobenzidine tetrahydrochloride was used as chromogen. Slides were counterstained in hemotoxylin. To quantify DNA synthesis, the percentage of nuclear profiles that stained for 
PCNA was determined for the tunica media.

To evaluate changes in vascular smooth muscle cell phenotype during organ culture, immunohistochemistry for vascular smooth muscle cell specific phenotype markers was performed on cross sections. These markers included muscle alpha-and gamma-actin (antibody HHF 35)(Tsukada et al, 1987), smooth muscle alpha-and gamma-actin (antibody CGA 7)(Gown et al, 1985), smooth muscle desmin (Ramaekers et al, 1983), and smooth muscle alpha-actin (antibody SM1)(Skalli et al, 1986).

\section{Vasoconstrictor infusion in vivo}

The experiments were performed in 6 week old male normotensive WKY rats and age matched SHR. Four groups of 8 rats each were defined. Three groups consisted of WKY rats that received either saline, phenylephrine (2 $\mathrm{mg} / \mathrm{kg} /$ day $)$ or angiotensin II $(0.3 \mathrm{mg} / \mathrm{kg} /$ day $)$ infused continuously during four. days through a subcutaneously implanted osmotic minipump (Alzet, model 2001, Alza, Palo Alto, CA, USA). One group consisted of SHR that received saline. Simultaneously all animals were provided with a second minipump, releasing the thymidine analogue BrdUrd at $24 \mu \mathrm{mol} / \mathrm{kg} /$ day. Body weight, heart rate, and systolic blood. pressure, as determined by tail-cuff plethysmography, were monitored during the experiment.

At the end of the experiment the vessels and organs of interest were immersion-fixed with $4 \%$ phosphate buffered formaldehyde and embedded in paraffin. Cross-sections were prepared for histology and immunocytochemistry as described above (section: structural changes).

\section{Mediators}

To evaluate whether arterial growth responses were induced by specific: arterial smooth muscle cell growth affecting factors, experiments were performed in the presence of specific receptor antagonists.

Bioassay experiments were performed in order to evaluate i) the stability' of vasoactive substances supplemented to the culture medium and ii) the presence of stable vasoactive mediators in media conditioned by arteries. For this purpose, harvested media (1 or $2.5 \mathrm{ml}$ ) were mixed with KRB (10 or 7.5 $\mathrm{ml}$ ) and added to arterial segments that were mounted in an. isometric myograph. The presence of a contractile factor was evaluated by introduction of specific antagonist(s), thereby blocking its contractile effect.

Arterial polyamine levels, putrescine, spermidine and spermine, were determined in homogenates of arterial segments by high-performance liquid chromatography of their benzoyl derivates (Verkoelen et al, 1988). In brief, 


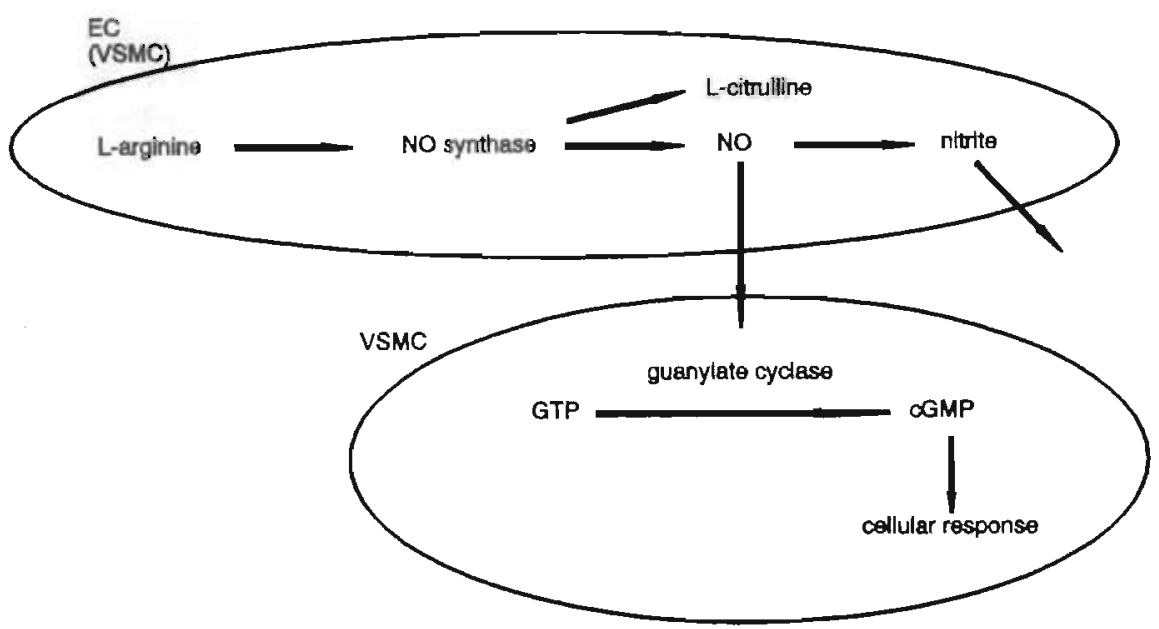

Figure 2.3 The L-arginine nitric oxide pathway. Nitric oxide (NO) is generated by nitric oxide synthase enzyme (NO synthase) in endothelial cells (EC) and vascular smooth muscle cells (VSMC). NO synthases can be measured by L-citrulline and $\mathrm{NO}_{2}$ production.

arterial preparations were powdered in liquid nitrogen, suspended in $0.5 \mathrm{ml}$ $0.3 \mathrm{M}$ perchloric acid, and homogenized (Ultra-Turrax). The supernatant obtained after centrifugation, $4000 \mathrm{~g}$ during $10 \mathrm{~min}$, was stored until determination of the polyamines. The pellet was used to measure the protein content according to Bradford (Bradford, 1976). Benzoyl derivates of the polyamines were separated on 2 reversed phase Lichrosorb $\mathrm{C}_{18}$ columns $(100$ $\mathrm{mm} \times 3 \mathrm{~mm})$ and eluted with methanol-water $(56: 44$, vol/vol). They were quantitated by ultraviolet absorbance $(229 \mathrm{~nm})$ using 1,6-hexanediamine as internal standard.

To estimate the NO production by vascular tissue (fig. 2.3), a nitrite assay was used. Tissue culture medium nitrite concentrations were determined in triplicate using the Griess reagent (Stuehr and Nathan, 1989). Griess reagent (500 $\mu \mathrm{l} ; 0.5 \%$ sulfanilamide and $0.05 \% \mathrm{~N}$-(1-naphthyl)ethylene-diamine dihydrochloride) was added to $500 \mu \mathrm{l}$ aliquots of media. After 10 minutes optical densities were measured at $550 \mathrm{~nm}$. By using known concentrations of $\mathrm{NaNO}_{2}$, a standard curve was constructed from 1 to $100 \mu \mathrm{M}$ nitrite. The information obtained in this type of experiments was supplemented with complementary observations during culture experiments performed in Larginine free culture media with and without addition of L-arginine analogues 
that have been reported to inhibit NO synthase (fig. 2.3).

\section{Tonic tone and arterial organ culture}

Continuous recording of contractile reactivity in isolated blood vessels, during a three day period, was performed in an isometric myograph under sterile conditions (fig.2.2). Arterial segments that were either left intact or were denuded of endothelium were mounted horizontally between an isometric force transducer (Grass UC3, Hugo Sachs, FRG) and a displacement device. The arterial preparations were stretched to their optimal lumen diameter, as determined by previous studies, to avoid stimulation of the vessels before starting the experiment. The organ chamber was filled with basal medium supplemented with 5\% human CMS-PDS in the absence or presence of the vasoactive substance. The medium was automatically replaced every $6 \mathrm{hr}$ during 3 days of culture, whereby a computer simultaneously started a vacuum pomp which removed the old medium and an infusion pomp that administered preheated $\left(37^{\circ} \mathrm{C}\right)$ fresh medium. The organ chamber of the myograph was covered with a transparent plastic box, wherein an atmosphere of $95 \%$ air and $5 \% \mathrm{CO}_{2}$ was maintained.

At the end of tension recording, mechanical and pharmacological properties were recorded in the presence of medium and after replacement of the medium by KRB.

\section{SERA AND PHYSIOLOGICAL SALT SOLUTIONS}

The Krebs-Ringer physiological salt solution (KRB), used primarily in myograph experiments, had the following composition (mmol/l): $\mathrm{NaCl} 118.5$; $\mathrm{KCl} 4.7 ; \mathrm{MgSO}_{4} 1.2 ; \mathrm{KH}_{2} \mathrm{PO}_{4} 1.2 ; \mathrm{NaHCO}_{3} 25.0 ; \mathrm{CaCl}_{2} 2.5 ;$ glucose 11.1. $\mathrm{KRB}$ without the addition of $\mathrm{NaHCO}_{3}$, used for sympathectomy of vessels, was set at $\mathrm{pH}=4.0$ with glutathione. High potassium solution (K-KRB $125 \mathrm{mmol} / \mathrm{l}$ ), was prepared by replacing all $\mathrm{NaCl}$ in normal $\mathrm{KRB}$ with an equimolar amount of $\mathrm{KCl}$.

Commercially available fetal calf serum (Seralab, Sussex, UK) appeared to contain high concentrations of serotonin (5-HT)(De Mey et al, 1989). For this reason, the serum was dialyzed tree times within $24 \mathrm{hr}$ against a phosphate buffer $\left(\mathrm{KH}_{2} \mathrm{PO}_{4}-\mathrm{K}_{2} \mathrm{HPO}_{4}-\mathrm{NaCL} ; \mathrm{pH} 7.4\right)$. The serum was then filtered, heated at $56^{\circ} \mathrm{C}$ for 30 minutes and stored at $-70^{\circ} \mathrm{C}$.

Rat WBS (Vogel et al, 1978) was prepared by collecting blood into syringes containing sodium citrate (final concentration, $0.38 \% \mathrm{wt} / \mathrm{vol}$ ). The anticoagulated blood was transferred to plastic centrifuge tubes and $1.0 \mathrm{M}$ $\mathrm{CaCl}_{2}$ was added to a final concentration of $14 \mathrm{mM}$. The blood was incubated 
at $37^{\circ} \mathrm{C}$ for $2 \mathrm{hr}$ and centrifuged at $3000 \mathrm{rpm}$ at $4^{\circ} \mathrm{C}$ for $15 \mathrm{~min}$. The supernatant was spun at $13500 \mathrm{rpm}$ at $4^{\circ} \mathrm{C}$ for 30 minutes. It was then heated to $56^{\circ} \mathrm{C}$ for 30 minutes, centrifuged at $13500 \mathrm{rpm}$ for 20 minutes at $4^{\circ} \mathrm{C}$, and filtered. The WBS was stored at $-70^{\circ} \mathrm{C}$.

Both rat PDS and human or rat CMS-PDS (Vogel et al, 1978) were prepared by collecting blood in prechilled syringes that contained sodium citrate to prevent coagulation (final concentration of sodium citrate, $0.38 \%$ $\mathrm{wt} / \mathrm{vol})$. The anticoagulated blood was transferred to prechilled plastic centrifuge tubes and spun at $3000 \mathrm{rpm}$ for 18 minutes at $4^{\circ} \mathrm{C}$. The supernatant was respun at $13500 \mathrm{rpm}$ for 20 minutes at $4^{\circ} \mathrm{C}$ to remove the platelets. After the second spun, $1.0 \mathrm{M} \mathrm{CaCl}_{2}$ was added to a final concentration of $20 \mathrm{mM}$, and KRB was then added in a ratio of 1 vol to 6 vol plasma. The plasma then was aliquoted into centrifuge tubes and incubated at $37^{\circ} \mathrm{C}$ for $2 \mathrm{hr}$ to allow clot. formation. The tubes were spun at $13500 \mathrm{rpm}$ for 30 minutes at $4^{\circ} \mathrm{C}$. The supernatant, PDS, was dialyzed against $0.1 \mathrm{M}$ Tris- $\mathrm{HCl}, \mathrm{pH} 7.4$, at $4^{\circ} \mathrm{C}$ for $24 \mathrm{hr}$.

Part of this PDS-fraction was applied to a CM Sephadex C-50 column $\left(50 \mathrm{~cm} * 2 \mathrm{~cm} ; \mathrm{I}^{*} \mathrm{~d}\right)$ that had been equilibrated in $0.01 \mathrm{M}$ Tris- $\mathrm{HCl}, \mathrm{pH} 7.4 / 0.09 \mathrm{M}$ $\mathrm{NaCl} / 0.02 \mathrm{M}$ benzamiune. Approximately 8 times the volume of fluid that was applied to the column was collected. The eluate was concentrated back to the original volume in an Amicon concentrator with a PM 10 filter (cutoff, molecular weight 10000). The concentrated CMS-PDS was dialyzed against three changes of KRB solution over $24 \mathrm{hr}$. Both PDS and CMS-PDS were then heated to $56^{\circ} \mathrm{C}$ for 30 minutes, centrifuged at $13500 \mathrm{rpm}$ for 20 minutes, and filtered. PDS and CMS-PDS were stored at $-70^{\circ} \mathrm{C}$.

\section{CHEMICALS}

Indomethacin, 6-hydroxydopamine, 5-bromo-2'-deoxyuridine, angiotensin II, saralasin, $\mathrm{N}^{\mathrm{G}}$-nitro-L-arginine methyl ester (L-NAME) were obtained from Sigma Chemicals (St. Louis, Mo, USA). Sodium nitroprusside (SNP) and 5-HT creatinine sulphate (serotonin) were from Janssen Chemica (Beerse, Belgium). The homodimers of PDGF, and endothelin were obtained from Bachem (Bubendorf, Switzerland). Transforming growth factor beta and basic fibroblast growth factor were purchased from Peninsula Laboratories (St. Helens, UK). ${ }^{3} \mathrm{H}$-thymidine $(0.2 \mu \mathrm{M}, 5 \mathrm{Ci} / \mathrm{mmol})$ and ${ }^{14} \mathrm{C}$-leucine $(18 \mu \mathrm{M}, 54 \mathrm{mCi} / \mathrm{mmol})$ were obtained from Amersham (Amersham, UK). Insulin-like growth factor-1 was a kind gift of Dr. K. Hofbauer (Basle, Switzerland). Interleukin-1 was kindly supplied by Dr. W. Buurman (University of Limburg, Maastricht, The Netherlands). BQ123 (endothelin $\mathrm{ET}^{\mathrm{a}}$ receptor antagonist) was a gift of $\mathrm{E}$. Ohlstein (SKF, King of Prussia, PA, USA). 


\section{STATISTICS}

Statistical significance of differences between data of experimental groups that were normally distributed and had comparable variances, was evaluated where applicable by one- or two-way analysis of variance. For multiple preplanned comparison, the analysis was followed either by Bonferroni's $t$-test or Scheffe's F-test (Wallenstein et al, 1980; Sokal and Rolf, 1981). Data are then shown as means \pm SE or means \pm SEM. $\mathrm{P}<0.05$ was accepted to denote statistical significance of differences. Statistical significance of differences between data of experimental groups that were not normally distributed and variances differed significantly (Bartlett's X-test), data were transformed as indicated by a Box-Cox procedure and compared by analysis of variance (Sokal and Rolf, 1981). For multiple comparisons, the analysis was followed by Scheffe's F-test. In this statistical analysis, data are shown as means of untransformed or retransformed data with $95 \%$ confidence intervals. $\mathrm{P}<0.05$ was selected as the level of statistical significance. 



\section{Chapter}

DNA Synthesis in Isolated Arteries. Kinetics and Structural Consequences

H.C.M. Boonen, P.M.H. Schiffers, G.E. Fazzi, G.M.J. Janssen, M.J.A.P. Daemen and J.G.R. De Mey

Departments of Pharmacology and ${ }^{\mathrm{a}}$ )Pathology, University of Limburg, Maastricht, the Netherlands.

(American Journal of Physiology 1991, 260: H210-H217) 


\section{INTRODUCTION}

Synthesis of DNA in the arterial wall of adult individuals is low but can increase in response to elevated blood pressure and injury (for review, see Schwartz et al, 1986). This increase is transient for cells within the arterial media (McLean and Nakaone, 1974; Carlier et al, 1983; Loeb et al, 1986; Majesky et al, 1987). In the case of arterial injury, this increase could be due to the transient nature of the stimulus, i.e., the injury itself or the supply of exogenous growth factors (Ross, 1986; Schwartz et al, 1986; Clowes et al, 1989). Arterial smooth muscle cells, however, that migrated into the intima after mechanical injury, continue to proliferate for prolonged periods of time (Clowes et al, 1983 ; Schwartz et al, 1986). In hypertension, the proliferative response could be limited once the resulting increased medial thickness normalizes medial stress (Folkow, 1982). Yet, arterial smooth muscle cells isolated from spontaneously hypertensive rats proliferate significantly faster in culture than cells isolated from arteries of normotensive rats (Yamori et al, 1981; Kanbe et al, 1983). Increased DNA synthesis in the arterial media following injury in vivo does not lead to medial hyperplasia or hypertrophy (Clowes et al, 1980; Clowes et al, 1983 ${ }^{b}$ ). Structural consequences of hypertension may include hypertrophy, hyperplasia, and hyperploidy of the media (Owens and Schwartz, 1982; Owens and Schwartz, 1983; Owens and Reidy, 1985; Schwartz et al, 1986). The reasons for the transient nature of intra-arterial DNA synthesis in vivo and for the different structural consequences are unclear. They could be due to the presence of growth inhibitory influences within the arterial wall of adult individuals.

To evaluate this possibility, we recorded in the present study the time course and structural consequences of DNA synthesis in isolated arterial segments that were continuously exposed to serum growth factors under constant mechanical conditions. The experiments were performed in tissue culture to combine some of the advantages of two classical experimental approaches of arterial smooth muscle growth control, i.e., arterial injury in situ and enzymatically dispersed smooth muscle cells in culture. The advantages include (1) continuous presence of growth stimuli; (2) possibility to mechanically stress the tissue, (3) easy recording of changes in contractile reactivity accompanying growth responses, (4) possibility to evaluate the interaction between different arterial cell types, (5) study of growth responses of arterial cells at normal density and in the presence of their natural micro-environment, (6) possibility to evaluate structural changes, and (7) applicability to a wide variety of vascular preparations (Mauger et al, 1975; DiCorletto et al, 1983; De Mey et al, 1989). 


\section{MATERIALS AND METHODS}

The experiments were performed on renal arteries that had been isolated from 20-week old male Wistar-Kyoto rats. The renal arteries were sympathectomized using $300 \mu \mathrm{g} / \mathrm{ml}$ 6-hydroxydopamine during $10 \mathrm{~min}$ (Aprigliano and Hermsmeyer, 1976) and were mechanically denuded of endothelium (De Mey and Brutsaert, 1984; De Mey et al, 1989), using the shaft of an hypodermic injection needle (diameter $0.9 \mathrm{~mm}$ ). The success of the latter was indicated by the absence of relaxing responses to acetylcholine (De Mey and Vanhoutte, $1982^{\mathrm{a}}$ ) and by the removal of all luminal cells that stained for Von Willebrand factor. For evaluation of contractile reactivity, DNA synthesis and arterial structure, 2-mm segments were cut from either the right or left renal artery. For determination of polyamine levels, $3-\mathrm{mm}$ segments were used.For the analysis of nuclear DNA content, the right and left renal artery of one animal were pooled together. Preliminary observations indicated that there were no significant differences between right and left renal arteries.

\section{Tissue culture}

Arterial segments were maintained in tissue culture as previously described (De Mey et al, 1989). The segments were individually suspended in 25 -mm culture wells filled with $2.5 \mathrm{ml}$ Dulbecco's Eagle's modified culture medium (GIBCO, Paisley, UK), containing $100 \mu \mathrm{g} / \mathrm{ml}$ streptomycin (Pharmachemie, Haarlem, The Netherlands), $100 \mathrm{IU} / \mathrm{ml}$ penicillin (Gist-Brocades, The Netherlands), $0.2 \mathrm{mM}$ glutamine (GIBCO) and $20 \%$ fetal calf serum (Seralab, Sussex, UK). The fetal calf serum, a classical but undefined source of growth factors, had been dialyzed to remove low molecular weight contractile factors (De Mey et al, 1989) and polyamines (Thyberg and Fredholm, 1987). Culture dishes were incubated for up to 2 wk at $37^{\circ} \mathrm{C}$ in an atmosphere of $95 \%$ air, $5 \% \mathrm{CO}_{2}$ at $97 \%$ humidity. With intervals of 2-3 days, the arterial segments were transferred to culture dishes filled with fresh culture medium. After 1-4 or 14 days, some of the segments were removed from culture.

To estimate the extent of DNA synthesis, $1 \mu \mathrm{M}$ of the thymidine analogue, 5-bromo-2'-deoxyuridine (BrdUrd, Sigma Chemicals, St. Louis, MO, USA) was included in the medium during the last $24 \mathrm{~h}$ of the culture. Nuclear incorporation of BrdUrd was later evaluated by immunohistochemistry on cross-sections (Folkow, 1982; Gratzner, 1982; De Mey et al, 1989).

Most of the culture experiments were performed with renal arterial segments of Wistar-Kyoto rats mounted on a 20-gauge hypodermic needle (diameter $0.9 \mathrm{~mm}$ ). This allowed us to maintain arterial diameter and transmural pressure close to those expected in vivo (see Results section and De Mey et al, 1989). As a consequence, however, medium was supplied only from 
the adventitial surface during arterial tissue culture. In one series of experiments, which was designed to evaluate effects of transmural pressure on intraarterial DNA synthesis in vitro, arterial segments from the sam rats were suspended for 3 days on supports with diameters between 0.25 and $1.12 \mathrm{~mm}$, resulting in equivalent transmural pressures ranging from hypo- to hypertensive levels (see Results section). The smallest support used $(0.25 \mathrm{~mm})$ was smaller than the unstressed diameter of the rat renal artery $(0.5-0.6 \mathrm{~mm})$ and did not limit the access of culture medium to the luminal surface of the arterial segments or prevent migration of cells into the lumen.

\section{Contractile reactivity}

Renal arterial segments that had been freshly isolated or cultured were mounted horizontally in an organ chamber between an isometric force transducer (Kistler Morse DSC 6 or Statham UC2) and a displacement device. The organ chamber was filled with $25 \mathrm{ml}$ Krebs-Ringer bicarbonate solution that was maintained at $37^{\circ} \mathrm{C}$ and aerated with $95 \% \mathrm{O}_{2}, 5 \% \mathrm{CO}_{2}$. All arterial preparations were placed at optimal diameter for mechanical performance using stepwise stretching and intermittent activation with high potassium solution (De Mey and Vanhoutte, 1982 ${ }^{\mathrm{a}}$; De Mey et al, 1989). Contractile responses were expressed as increases in wall tension (increases in force per twice the segment length). The Krebs-Ringer bicarbonate solution had the following composition (mmol/l): $118.3 \mathrm{NaCl}, 4.7 \mathrm{KCl}, 2.5 \mathrm{CaCl}_{2}, 1.2 \mathrm{MgSO}_{4} \cdot \mathrm{H}_{2} \mathrm{O}, 1.2 \mathrm{KH}_{2} \mathrm{PO}_{4}$, $25.0 \mathrm{NaHCO}_{3}$, and 11.1 glucose. For high potassium solution, all $\mathrm{NaCl}$ was replaced by an equimolar amount of $\mathrm{KCl}$. Recording of contractile reactivity was terminated by exposing resting arterial preparations at optimal diameter and $37^{\circ} \mathrm{C}$ to periodate-lysine-paraformaldehyde fixative (McLean and Nakaone, 1974). The fixated arterial segments were removed $30 \mathrm{~min}$ later from the recording apparatus and stored in phosphate-buffered saline at $4^{\circ} \mathrm{C}$ until they were processed for histological examination.

\section{Histology and immunohistochemistry}

Fixated arterial segments were routinely processed and embedded in paraffin. Cross-section were stained with hematoxylin and eosin for morphometrical analysis. The area enclosed by the internal and external elastic lamina will be referred to as the media. The area abluminally from the external elastic lamina is referred to as adventitia. In arterial segments that had been maintained in tissue culture for $2 \mathrm{wk}$, a third layer could be clearly discerned. This layer was situated at the outer abluminal edge of the preparations and will be referred to as periadventitia. To estimate the number of cells of the arterial layers, we counted the number of nuclear profiles in each layer of a cross- 
section. Lumen radius ( $\mathrm{r}$ ) and media cross-sectional area (CSA) were measured using standard morphometric techniques (MOP video-plan). An average media thickness (T) of an hypothetical circular arterial cross-section was computed using the formula CSA $=\pi(r+T)^{2}-\pi r^{2}$. For tissues that had been cultured in the presence of BrdUrd, an indirect enzyme-labeled antibody technique with peroxidase-conjugated second-step reagent was used to visualize nuclei that had incorporated the thymidine analogue (Gratzner, 1982). To quantify the incorporation of BrdUrd, the percentage of nuclei that stained for peroxidase was counted in each arterial layer.

In an attempt to evaluate migration and changes in phenotype of arterial smooth muscle cells during arterial tissue culture, we performed immunohistochemistry for a number of markers on cross-sections of arterial segments that had been freshly isolated or cultured for up to 2 wk. For this purpose, antibodies against collagen IV, muscle alpha- and gamma-actin (antibody HHF 35 ), and smooth muscle alpha-actin (antibody SM1) were used.

\section{Measurement of DNA/nucleus}

The proportion of nuclei in cultured arteries that contained a diploid or tetraploid DNA content was measured by flow cytometry on isolated nuclei that had been prepared by enzyme digestion and stained with the fluorescent DNA dye propidium iodide (Owens and Schwartz, 1983).

\section{Arterial polyamine levels}

Levels of putrescine, spermidine, and spermine were determined in homogenates of freshly isolated and cultured arterial segments by reversed phase high-performance liquid chromatography (HPLC), as described by Verkoelen et al (1988). In brief, arterial preparations were powdered in liquid nitrogen, suspended in $0.3 \mathrm{M}$ perchloric acid, and homogenized (Ultra-Turrax). The supernatant obtained after centrifugation $(4,000 \mathrm{~g}$ during $10 \mathrm{~min})$ was stored at $-20^{\circ} \mathrm{C}$ until determination of the polyamines. The pellet was used to measure the protein content according to Bradford (Blank et al, 1988). Benzoyl derivatives of the polyamines were separated on 2 Lichrosorb HPLC columns $(100 \mathrm{~mm} \times 3 \mathrm{~mm})$ packed with $\mathrm{C}_{18}$ reversed phase material and eluted with methanol-water $(56: 44, \mathrm{vol} / \mathrm{vol})$ hey were quantitated by ultraviolet absorbance (229 $\mathrm{nm}$ ), using 1,6-hexanediamine as internal standard. 


\section{Statistics}

Data are shown as means $\pm S E(n=6-24$ arteries). Statistical significance of differences was evaluated by one- or two-way analysis of variance where appropriate. For multiple preplanned comparisons, the analysis of variance was followed by Bonferroni's t-test (Wallenstein et al, 1980; Sokal and Rohlf, 1981).

\section{RESULTS}

\section{Contractile reactivity}

Freshly isolated renal arterial segments developed maximal contractile responses to $125 \mathrm{mM}$ potassium $(3.2 \pm 0.4 \mathrm{mN} / \mathrm{mm})$ when stretched to an average lumen diameter of $1.12 \pm 0.03 \mathrm{~mm}(\mathrm{n}=24)$. From the results of the resting wall tension at this diameter $(7.3 \pm 0.6 \mathrm{mN} / \mathrm{mm})$ and Laplace's law (transmural pressure $=$ wall tension/radius), equivalent transmural pressure in resting renal arteries averaged $13.1 \pm 0.99 \mathrm{mN} / \mathrm{mm}^{2}(\mathrm{n}=24)$. This calculated pressure is in the range of the pressure that the arterial wall is exposed to in vivo. Sliding the arterial segments over a needle (diameter $0.9 \mathrm{~mm}$ ) effectively removed all endothelium. It also reduced maximal contractile responses to 125 $\mathrm{mM}$ potassium to $2.2 \pm 0.4 \mathrm{mN} / \mathrm{mm}(\mathrm{n}=17)$.

Up to 4 days of tissue culture did not decrease contractile responses to $125 \mathrm{mM}$ potassium in renal arterial segments that had been sympathectomized, denuded of endothelium, and maintained at a diameter of $0.9 \mathrm{~mm}$ during culture (fig. 3.1). If anything, contractile reactivity recovered from the reduction that initially accompanied mechanical removal of endothelium. After 14 day of culture, however, responses to $125 \mathrm{mM}$ potassium were reduced to $22 \pm 6 \%$ of those observed in freshly isolated preparations.

Figure 3.2 illustrates mechanical properties of renal arterial segments that had been suspended at different diameters during 3 days of tissue culture. Since the culture conditions that were used induce only a small contraction and do not alter the stiffness of the arterial wall (De Mey and Vanhoutte, 1982 ${ }^{\mathrm{a}}$ ), segments mounted on a different diameter were continuously exposed to a different transmural pressure. This ranged from a hypotensive to hypertensive level (fig. 2). Only the latter resulted in a significant reduction of contractile responses to $125 \mathrm{mM}$ potassium following culture. 

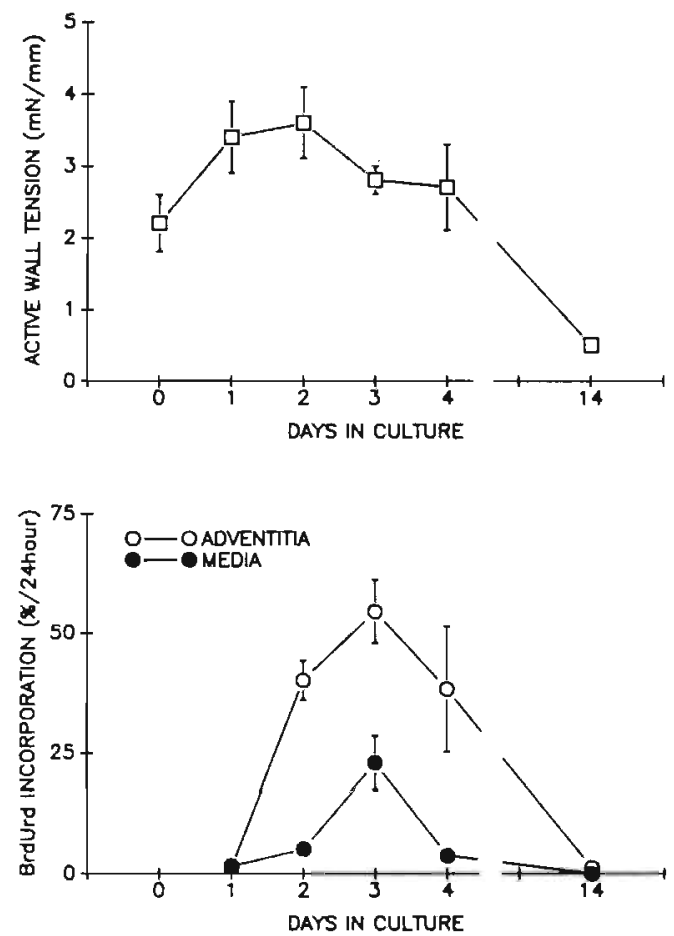

Figure 3.1 Effects of tissue culture on contractile responses to $125 \mathrm{mM}$ potassium (top) and DNA synthesis (bottom) in rat renal artery. Arterial segments were isolated from adult Wistar-Kyoto rats, chemically sympathectomized, mechanically denuded of endothelium, and suspended on a support (diameter $0.9 \mathrm{~mm}$ ) in culture medium supplemented with $20 \%$ dialyzed fetal calf serum. During the last $24 \mathrm{~h}, 1 \mu \mathrm{M}$ 5-bromo-2'-deoxyuridine (BrdUrd) was included in culture medium. After 1-14 days of culture, arterial segments were mounted at optimal diameter and their active wall tension in response to $125 \mathrm{mM}$ potassium was recorded. They were subsequently fixed and nuclear incorporation of BrdUrd was evaluated by immunohistochemistry on cross-sections. Data are shown for media and adventitia. All data are shown as means $\pm S E$ ( $n=6-18$ segments).

\section{Intra-arterial DNA synthesis}

In freshly isolated arterial segments, low levels of putrescine, spermidine, and spermine could be detected (fig. 3.3). These polyamines play a key role in the synthesis of protein and DNA in a wide variety of cell types (WhiteTabor and Tabor, 1984; Reidy and Silver, 1985). Arterial levels of polyamines 

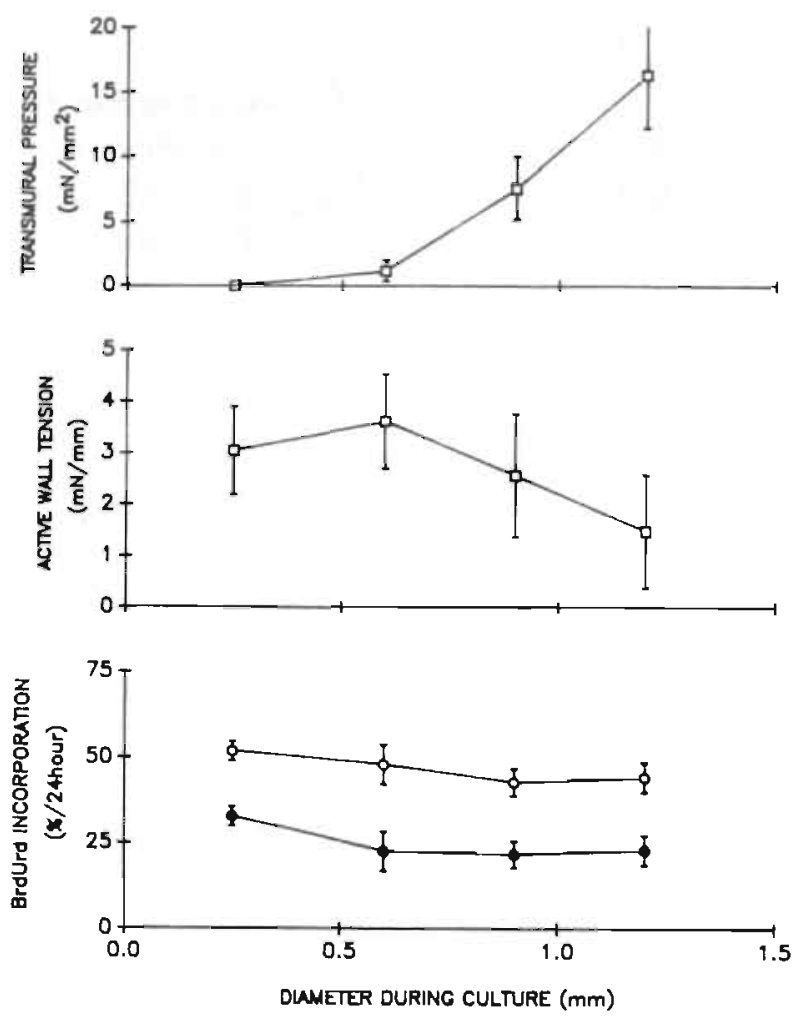

Figure 3.2 Effects of isometric distension during tissue culture on mechanical properties and DNA synthesis in rat renal artery. Arterial segments that had been sympathectomized and denuded of endothelium were maintained for 3 days in tissue culture at a diameter ranging between 0.25 and $1.20 \mathrm{~mm}$. During the last $24 \mathrm{~h}$ of culture, $1 \mu \mathrm{M}$ BrdUrd was included in culture medium. Equivalent transmural pressure (top) was calculated by Laplace's law using the diameter during culture and the resting wall tension at this diameter after culture. To record active wall tension (middle), arterial segments were made to contract in response to $125 \mathrm{mM}$ potassium an optimal diameter for mechanical performance. DNA synthesis during the third culture day (bottom) was estimated by immunohistochemistry for BrdUrd on cross-sections. These data are shown for media (closed circles) and adventitia (open circles). All data are shown as means $\pm S E(n=6-18$ segments).

were, however, not significantly affected by up to 4 days of tissue culture. There was tendency for putrescine to be transiently elevated after 1 day (fig. 3.3). The changes in the levels of spermidine, which fell within the limits of interindividual variability and experimental error, tended to be delayed and to 


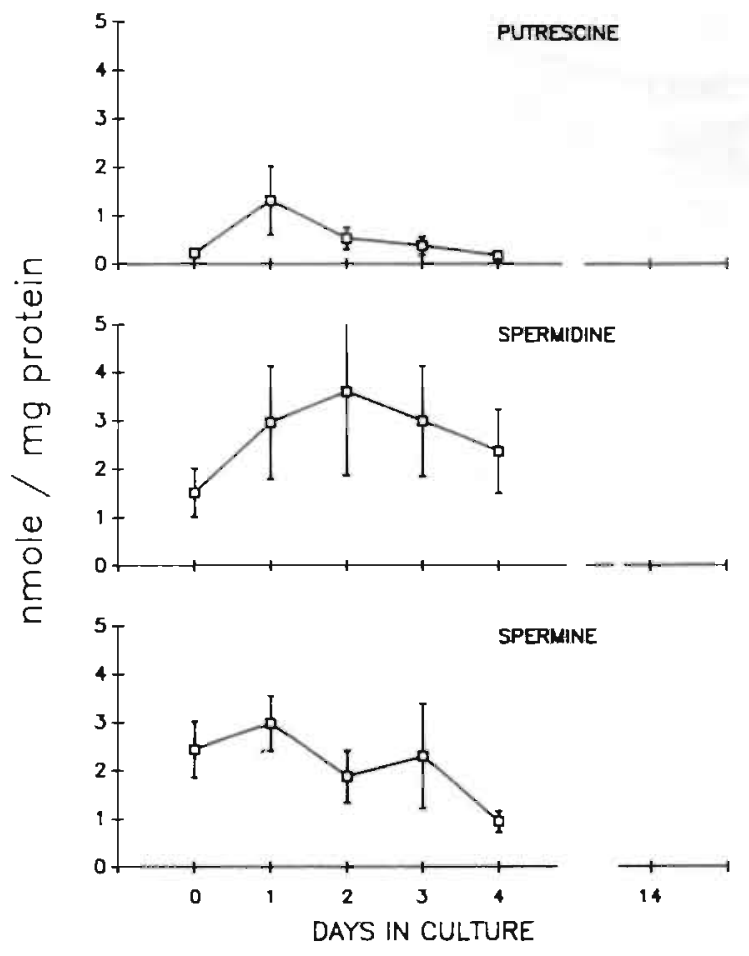

Figure 3.3 Effects of tissue culture on levels of polyamines in rat renal artery. After 0-4 days of culture, levels of putrescine (top), spermidine (middle) and spermine (bottom) were determined by high-performance liquid chromatography in supernatants of homogenates. Data are shown as means \pm SE $(n=6-13)$. For neither polyamine did the effect of time reach statistical significance.

be more gradual (fig. 3.3).

Judging from nuclear incorporation of BrdUrd, tissue culture stimulated DNA synthesis in both the medial and adventitial layer of the isolated arterial preparations (figs. 3.1, 3.2 and 3.4). There was a latency of approximately 1 day after which BrdUrd incorporation was evident. It was maximal during the third culture day (fig. 3.1). In the media, BrdUrd labeling at this point averaged $31.4 \pm 4.1 \% / 24 \mathrm{~h}$ but fell thereafter and remained low for the remainder of the study (figs. 3.1 and 3.4). In the original adventitia, the percentage of cells incorporating BrdUrd was significantly larger at each individual time than in the media (fig. 3.1). In the adventitia, incorporation of BrdUrd was 


\section{CHAPTER 3}
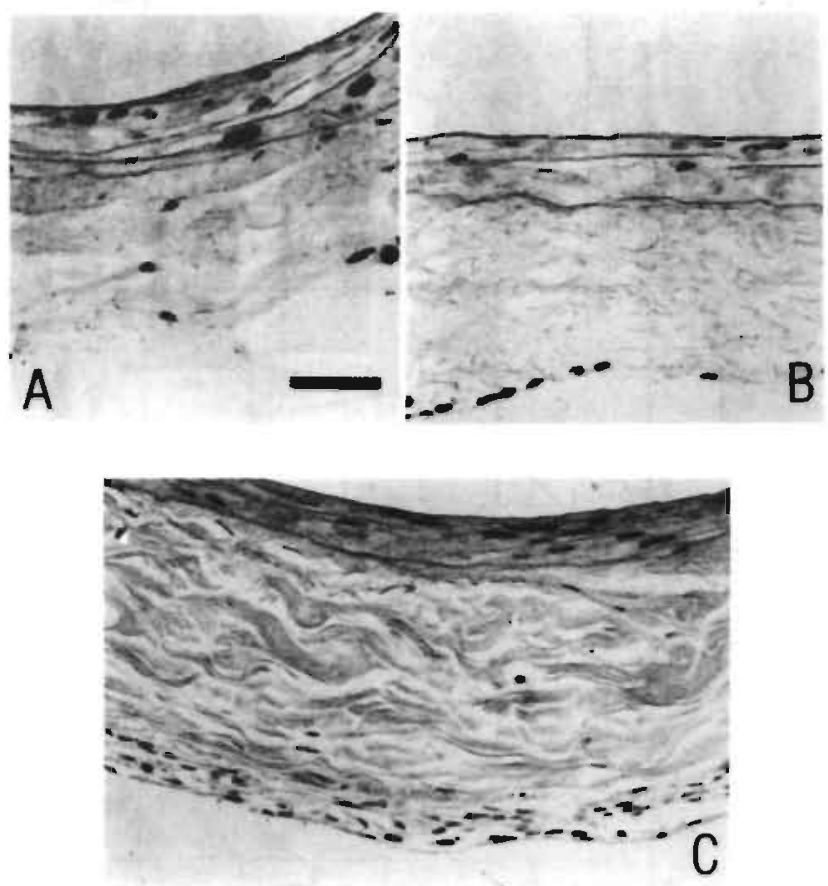

Figure 3.4 Cross-sections of renal artery segments immunohistochemically stained for BrdUrd and lightly counterstained with hematoxylin. Preparations had been maintained in tissue culture for 3 (A), 4 (B), and 14 (C) days and had been exposed to BrdUrd for the last $24 \mathrm{~h}$. Dark protiles represent nuclei in which DNA was synthesized during the last $24 \mathrm{H}$. Bar represents $50 \mu \mathrm{m}$. Arterial lumen is at top.

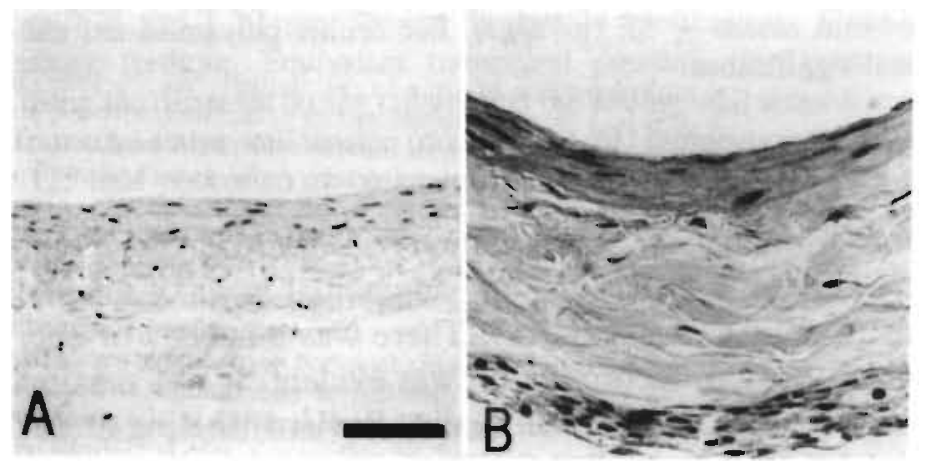

Figure 3.5 Cross-sections of renal arterial segments stained with hematoxylin-eosin. A: freshly isolated preparation; B: preparation that had been maintained in tissue culture for 14 days. Bar indicates $50 \mu \mathrm{m}$. Arterial lumen is at the top. 

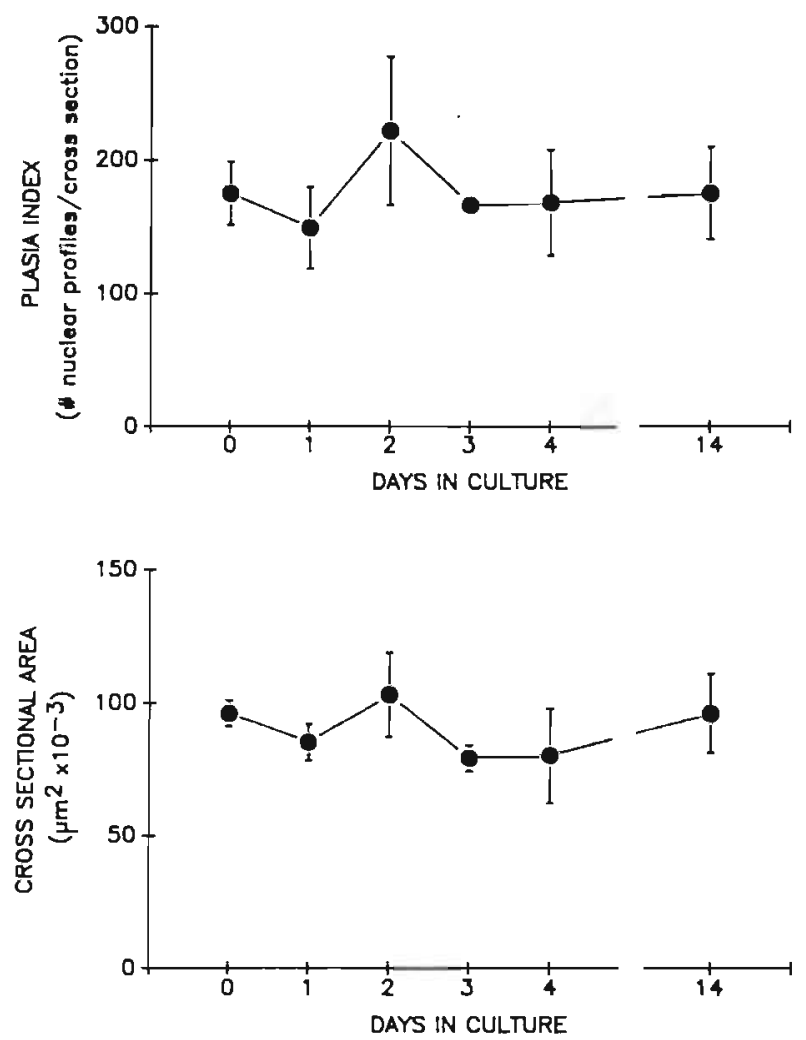

Figure 3.6 Effects of tissue culture on plasia (top) and cross-sectional are a (bottom) of media in renal arterial segments. As an index of plasia, number of nuclear profiles per cross-section is shown. All data are shown as means $\pm S E$ ( $n=6$ segments).

maintained longer than in the media but also fell to a low level during prolonged culture (fig. 3.1).

Figure 3.2 illustrates the extent of nuclear incorporation of BrdUrd during the third culture day in arterial segments continuously maintained at a different diameter and exposed to different transmural pressures. Isometric distension during tissue culture did not promote DNA synthesis in the arterial media or adventitia in addition to the stimulation observed during culture alone (fig. 3.2). The arterial lumen was obstructed by the impalement of tissue on needles of $0.6 \mathrm{~mm}$ or larger; however, there was no lumen obstruction when 


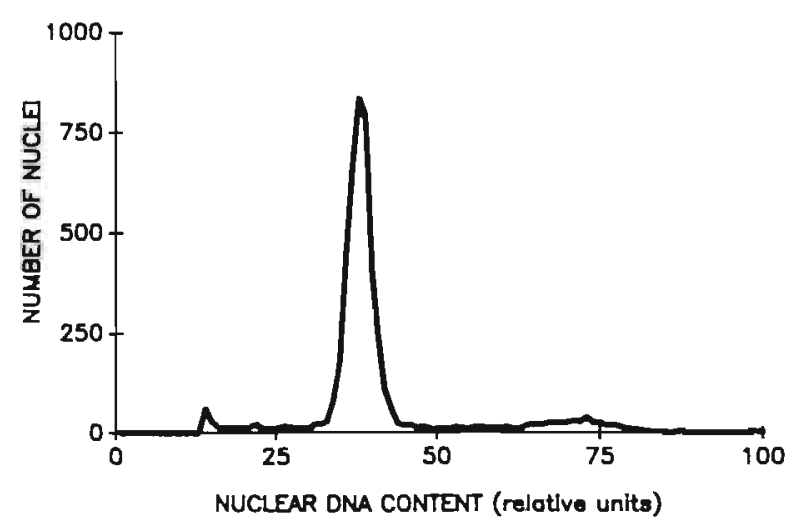

Figure 3.7 Nuclear DNA content in rat renal arteries that had been maintained in tissue culture for 6 days. Right and left renal artery of a rat had been sympathectomized, mechanically denuded of endothelium, and maintained in tissue culture at a diameter of 0.9 mm for 6 days. A nuclear suspension was prepared by enzymatic digestion and nuclear DNA content was measured by flow cytometry using fluorescent dye propidium iodide. Histogram displays a number of nuclei vs. nuclear DNA content and is representative for 4 separate experiments.

a support of $0.25 \mathrm{~mm}$ diameter was used. Similarity of nuclear incorporation of BrdUrd at all diameters (fig. 3.2) therefore implies that the arterial wall did not offer a significant diffusion barrier for the mitogens that were present in the culture medium.

\section{Structural changes}

Tissue culture for up to 14 days did not alter the overall architecture of the arterial media and adventitia but gave rise to the formation of a new layer at the adventitial edge of the arterial preparations (figs. 3.4 and 3.5). Despite significant stimulation of DNA synthesis, tissue culture did not alter the crosssectional area of the media (fig. 3.6), nor did tissue culture, as judged from the number of nuclear profiles per cross-section, affect the number of medial cells (fig. 3.6). We considered that tissue culture might have resulted in the formation of hyperploid nuclei. Figure 3.7 illustrates DNA content in nuclear suspensions prepared from arteries that had been maintained in tissue culture for 6 days. From the results in figure 3.1, the tissue from which the nuclei were prepared had been taken through a transient stimulation of DNA 


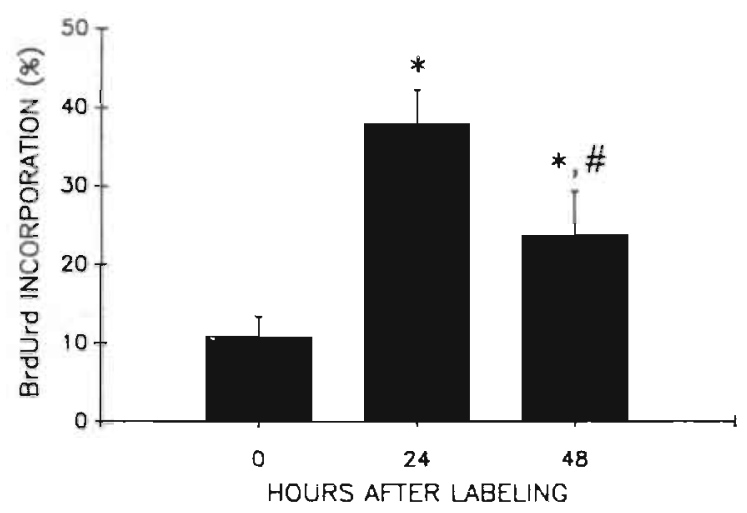

Figure 3.8 Changes with time of presence of BrdUrd in medial nuclei of renal arterial segments during culture. Arterial segments that had been sympathectomized and denuded of endothelium were exposed to $1 \mu \mathrm{M}$ BrdUrd during 52nd hour of tissue culture. They were fixated immediately after labeling or after 24 or $48 \mathrm{~h}$ of incubation in culture medium without BrdUrd. Percentage of labeled nuclei in arterial media is shown as means \pm SE ( $n=6$ segments). Difference for observations after $0\left(^{*}\right)$ and $24(\not) h$ of delay is statistically significant $(p<0.05)$.

synthesis. The majority of the nuclei, however, contained a diploid amount of DNA (fig. 3.7). In the histogram, $<2 \%$ of the nuclei fall under the tetraploid area of the curve based on DNA content. This could be indicative of the level of DNA synthesis that persists after 6 days in culture rather than of true hyperploidy.

To solve the discrepancy between increased DNA synthesis in the arterial media on the one hand and the absence of medial hyperplasia and hyperploidy on the other hand, we evaluated the evolution with time of BrdUrd incorporated in the nuclei of medial cells. The results of these pulse-chase experiments are summarized in figure 3.8. During the 52nd hour of culture, $10.8 \pm 2.6 \%$ of the medial nuclei incorporated BrdUrd. When the arterial segments were subsequently incubated for $24 \mathrm{~h}$ in label-free medium, the percentage of labeled medial nuclei increased to $37.8 \pm 4.3 \%$. If the 1 -h labeling period was followed by $48 \mathrm{~h}$ of incubation in the absence of BrdUrd, the percentage of labeled nuclei dropped again to $23.7 \pm 5.7 \%$. When taken together, these observations indicate that DNA synthesis was rapidly followed by nuclear division and suggest that with time, labeled cells were lost from the media. One possibility is that these cells migrated out of the original muscular layer.

In cross-sections of arterial segments that had been cultured for longer 
periods of time (14 days), a third layer was clearly present in addition to the original media and adventitia (fig. 3.5). This newly formed structure was situated at the outer edge of the arterial segments. Each cross-section contained $704 \pm 119$ nuclear profiles that were organized in 3-6 continuous layers. In contrast to the original media and adventitia, the newly formed periadventitial structure displayed extensive DNA synthesis, $24 \pm 5 \%$ of the nuclei in the periadventitial layer incorporated BrdUrd during the 14th culture day. This DNA synthesis was restricted to the outermost cell layer (fig. 3.4).

The origin of the newly formed periadventitial structure can be traced back to the early culture period. After 2-3 days in culture, cells could be observed at the abluminal border of the arterial wall. These periadventitial cells were absent in freshly isolated preparations (fig. 3.5). The outgrowth of cells did not differ between preparations that had been maintained in culture for 3 days on supports ranging from 0.6 to $1.2 \mathrm{~mm}$. In preparations cultured on a thin non-obstructing support $(0.25 \mathrm{~mm})$, cellular growth occurred at both the adventitial and intimal surface.

To identify the nature and origin of the cells that formed the periadventitial layer, we performed a series of histological and immunohistochemical studies. In freshly isolated arterial segments, elastin was densely present in the media but absent in the adventitia. The presence of elastin in the media was little affected by tissue culture. In the newly formed periadventitial layer, as judged from cross-sections of arterial segments that had been cultured for 14 days, elastin was present in the deeper layers. In freshly isolated arteries, the presence of collagen IV, smooth muscle alpha-actin and muscle alpha- and gamma-actin was restricted to the media. In the media the content of collagen IV, smooth muscle alpha-actin and muscle alpha- and gamma-actin decreased with tissue culture after a delay of 2-4 days. Parts of the newly formed periadventitial layer in renal arteries that had been cultured for 14 days, however, contained collagen IV and actin. Immunoreactive smooth muscle alpha-actin could be visualized (antibody SM1) in $12.8 \pm 1.8 \%(n=6)$ of the periadventitial cells. SM1 positive cells were virtually restricted to the outermost edge of the newly formed cell layers. Surprisingly, the distribution of smooth muscle alpha-actin containing cells in the new layer coincided with that of DNA synthesizing cells (fig. 3.4). Immunoreactivity for muscle alphaand gamma-actin (antibody HHF35), on the other hand, could be visualized in $67.8 \pm 5.6 \%$ of the cells in the new layer and was uniformly distributed. 


\section{DISCUSSION}

Continuous exposure of isolated renal arterial segments to serum stimulated DNA synthesis. In the media, this response was transient and did not result in hyperplasia, hypertrophy, or hyperploidy. Instead it gave rise to the formation of a new arterial layer that displayed smooth muscle-like properties.

In arteries of adult animals, the turnover of smooth muscle cells is low. Arterial injury and interventions that lead to the development of hypertension can, however, stimulate synthesis of DNA in the arterial wall in vivo (for review, see Ross et al, 1986). This essential step in the proliferative response of cells is transient in the arterial media after both injury and hypertensive interventions (Carlier et al, 1983; Loeb et al, 1986; Majesky et al, 1987). Arterial smooth muscle cells that migrated into the intima following lumenal arterial injury continue to synthesize DNA and proliferate for at least 2 wk (Clowes and Clowes, 1980; Clowes et al, 1983 ${ }^{\mathrm{b}}$ ). Arterial smooth muscle cells that have been isolated by enzymatic digestion, continue to proliferate for prolonged periods of time in cell culture (Wallenstein et al, 1980; Yamori et al, 1981; Kanbe et al, 1983; White-Tabor and Tabor, 1984; Thyberg and Fredholm, 1987; Verkoelen et al, 1988). The different growth characteristics of isolated, intimal and medial arterial smooth muscle cells could be due to the transient and local character of the growth stimuli in vivo.

We therefore evaluated in the present study the kinetics of DNA synthesis in isolated arterial segments during continuous exposure to exogenous mitogens in tissue culture (Mauger et al, 1975; Fingerle and Kraft, 1987; De Mey et al, 1989). The experiments were performed in renal arterial segments that had been sympathectomized and mechanically denuded of endothelium to focus on growth responses of the arterial media in the absence of substances released by adrenergic nerve endings and endothelial cells that can influence contractile and growth responses in arterial smooth muscle (Bevan and Tsuru, 1979; De Mey and Vanhoutte, 1982 ${ }^{\mathrm{a}}$; DiCorletto et al, 1983; Davies, 1986; Majesky et al, 1987).

From the results of $24 \mathrm{~h}$ labeling experiments, intra-arterial DNA synthesis was transient in vitro in the continuous presence of growth stimuli. This was particularly evident in the arterial media. After a lag time of $24 \mathrm{~h}$, a significant level of DNA synthesis was observed. It reached its maximum during the third day and fell precipitously thereafter. During this period, the contractile reactivity of the arterial segments was not reduced. In an earlier study, we observed that arterial contractile reactivity dropped with prolonged culture irrespective of the extent of DNA synthesis in the arterial media (De Mey et al. 1989). These observations are in contrast with the view derived from cell culture experiments and histochemical studies that arterial smooth 
muscle cells must be transformed from a contractile into a synthetic phenotype before DNA synthesis (Chamley-Campbell et al, 1981; Schwartz et al, 1986). This discrepancy may be due to the high cell density in the arterial media maintaining the muscle phenotype (Blank et al, 1988).

Both the time course and the extent of DNA synthesis that we observed in vitro are similar to those following luminal injury of the rat carotid artery in vivo (Clowes and Clowes, 1980; Reidy and Silver, 1985; Clowes et al, 1989). In this experimental animal model, which depends on both medial injury and the supply of growth factors from the blood (Ross, 1986; Ross et al, 1986; Schwartz et al, 1986), intra-arterial DNA synthesis is preceded by a marked but highly transient rise in the activity of ornithine decarboxylase (Majesky et al, 1987). This enzyme is the rate-limiting step in the synthesis of polyamines that play a key role in protein and DNA synthesis (Pegg and McCann, 1982; White-Tabor and Tabor, 1984). In arterial smooth muscle cells in cell culture, platelet-derived growth factor stimulates the activity of ornithine decarboxylase and the production of putrescine (Thyberg and Fredholm, 1987). Pharmacological inhibition of these effects prevents the proliferative response to the growth factor (Thvberg and Fredholm. 1987). These nhervatinne suggest that transiont formation of polyamines in response to injury could underlie transient DNA synthesis in situ. In the present study, however, we attempted to maintain both the mechanical and chemical stimuli constant during several days of culture and yet we observed transient stimulation of DNA synthesis despite the absence of significant changes of the arterial polyamine levels.

DNA synthesis can slow down when it is not followed by nuclear division. The resulting nuclear hyperploidy has previously been observed in aortas of spontaneously hypertensive rats (Owens and Schwartz, 1982; Owens and Reidy, 1985). However, no hyperploidy could be detected in the present in vitro study. In addition, the pulse-chase experiments that we performed with BrdUrd indicate that DNA synthesis in the media was followed by nuclear division.

Observations in the original arterial media contrast to those in the new layer that was formed during prolonged culture. In view of the presence of elastin, collagen IV and actin, this layer was composed of smooth muscle-like cells. In view of the discrepancy between DNA synthesis and nuclear division in the media on the one hand and the absence of media hyperplasia on the other hand, smooth muscle cells that migrated out of the media could have been at the origin of the new periadventitial layer. Less likely, but at present not excluded, is that also adventitial fibroblasts that were transformed into myofibroblasts (Skalli et al, 1989) contributed to this new layer. Unlike medial cells, smooth muscle-like cells in the periadventitial layer continued to synthesize DNA and proliferate for at least $2 \mathrm{wk}$. It is of interest that this DNA synthesis was not uniformly distributed but limited to the outermost cell layer. 
The responsiveness of smooth muscle cells in and outside the arterial media to the continuous presence of mitogens strongly suggests that the micro-environment formed by smooth muscle cells themselves has an inhibitory influence on their growth responses. The contributions of structural hindrance and inhibitory factors remain to be elucidated.

We propose that the arterial media is, unlike isolated arterial smooth muscle cells, refractory to exogenous growth factors. Comparisons of growth responses after various physical interventions in vivo (Reidy and Silver, 1985; Clowes et al, 1989) and in isolated arteries (DiCorletto et al, 1983; Fingerle and Kraft, 1987) suggests that an undefined and possible subtle medial injury can temporarily overcome the inhibitory influences within the arterial media.

Continuous isometric distension (up to $18 \mathrm{mN} / \mathrm{mm}^{2}$ ) did not increase the maximal extent of DNA synthesis in isolated renal artery segments. Acute isobaric distension of the rat carotid artery in situ $(300 \mathrm{~mm} \mathrm{Hg}$ or 40 $\mathrm{mN} / \mathrm{mm}^{2}$ ), however, markedly stimulated intra-arterial DNA synthesis (Clowes et al, 1989). Previous experiments reported by Hume (1980) indicated that isotonic distension stimulated DNA synthesis in rabbit central ear arteries during tissue culture. It should be pointed out, however, that in both previous studies, distension not only affected the media but also the integrity of the endothelium. Since the endothelium can affect DNA synthesis in the arterial media (Davies, 1986; DiCorletto et al, 1983; Fingerle and Kraft, 1987), endothelial injury accompanying arterial distension, might have promoted DNA synthesis in arterial smooth muscle in the previous studies. Alternatively, distension could affect the kinetics rather than the maximal extent of DNA synthesis in the arterial media.

While tissue culture of arterial segments of normotensive rats stimulated DNA synthesis, it did not affect the structure of the media. Instead, it gave rise to a new layer that displayed smooth muscle-like properties. In view of the number of periadventitial cells and their kinetics of DNA synthesis after 14 days of culture, even a small number of smooth muscle cells that migrated out of the media during the early culture period could have formed the basis of the new layer. This process could display similarities to those underlying the formation of the neointima following luminal arterial injury (Clowes et al, $1983^{\text {b}}$; Ross, 1986; Ross et al, 1986; Skalli et al, 1989). The main difference between both growth responses obviously is the site at which it occurred. Since there is a role for growth factors derived from the blood in the response of the arterial wall to luminal injury in situ (Ross, 1986; Ross et al, 1986), at least platelet-derived growth factor is a potent chemotactic agent (Grotendorst et al, 1981; Ross et al, 1986), and serum factors were only applied to the adventitial side of the arterial segments during most of the experiments in vitro, and since obstruction of the arterial lumen formed a structural hindrance for intimal outgrowth in vitro, intimal periadventitial smooth muscle proliferation in vivo 


\section{CHAPTER 3}

and in vitro stress the importance of chemical attraction and migration of cells.

In summary, continuous exposure of isolated renal artery segments to exogenous mitogens transiently stimulated DNA synthesis. It did not result in hypertrophy, hyperplasia, or hyperploidy of the arterial media. It rather gave rise to a new arterial layer that was composed of smooth muscle-like cells. These observations suggest that migration of smooth muscle cells and local influences that inhibit cellular proliferation responses can help to maintain a constant number of smooth muscle cells in the arterial media of adult animals. 


\section{Chapter}

\section{4}

Growth Responses in Isolated Elastic,

Muscular and Resistance-sized Arterial Segments of the Rat

J.G.R. De Mey, M.P. Uitendaal, H.C.M. Boonen, P.M.H. Schiffers and G.E. Fazzi

Department of Pharmacology, University of Limburg, Maastricht, the Netherlands. (Blood Vessels 1991, 28:372-385) 


\section{INTRODUCTION}

Structural alterations of the vascular wall play a role in atherosclerosis and hypertension (Folkow, 1982; Schwartz et al, 1986). While different types of structural changes occur during these diseases, different parts of the circulatory system differ in their susceptibility to modification. Elastic arteries are affected to a larger extent by atherogenic conditions than the microcirculation. Media hypertrophy in hypertension is prominent only upstream from true arterioles (Folkow, 1982; Bohlen and Lohach, 1978). In large arteries, it is primarily due to hypertrophy/hyperploidy of the arterial smooth muscle cells (ASMC) (Schwartz et al, 1986; Owens, 1989). In small resistance-sized arteries, on the other hand, it has been attributed to ASMC hyperplasia (Mulvany et al, 1985; Owens et al, 1988). The reasons for this regional heterogeneity are not clear. Phenomena such as regional differences in hemodynamics, nervous input and supply of exogenous mitogens, could play a role (Owens, 1988; Bevan and Tsuru, 1981). Also mechanisms that are endogenous to the vascular wall could be involved. The proliferative response of isolated ASMC depends on the phenotype and density of the cells and on the presence of extracellular matrix components (Schwartz et al, 1986; ChamleyCampbell et al, 1979, 1981; Blank et al, 1988). ASMC and endothelial cells can produce factors that affect growth responses in an autocrine or paracrine manner (Davies, 1986; Walker et al, 1986; Sjölund et al, 1988; Staiano-Coico et al, 1988; De Mey et al, 1991 ${ }^{\mathrm{a}}$ ). As judged from acute responses to vasodilator and vasoconstrictor substances, the pharmacological properties of the arterial wall differ between vascular beds and change with branching (Vanhoutte, 1978; De Mey and Vanhoutte, 1982 ${ }^{\mathrm{a}}$ ). This could also apply for growth-affecting agents. There are, however, no comparative studies available that directly demonstrate local individuality of growth responsiveness in the arterial system. In the case of the smaller branches of the circulatory system, and especially for those segments that regulate vascular resistance and local blood flow, there even seems to be no appropriate experimental approach available yet.

We therefore recorded in the present study the extent of DNA synthesis and structural changes in a broad variety of isolated arterial preparations. The experiments were performed in segments of elastic, muscular and resistancesized arterial segments during organoid culture (De Mey et al, 1991 a , 1989; Boonen et al, 1991). This allowed to compare maximal responses to exogenous growth factors under identical external conditions in large and small arterial preparations of different anatomical origin. Because the endothelium can act as a source of endogenous growth factors and growth inhibitors (Davies, 1986; Staiano-Coico et al, 1988: De Mey et al, 1991ª), the experiments were performed in the arterial segments both with and without the endothelium. 


\section{MATERIALS AND METHODS}

Experiments were performed in arterial segments that had been isolated from 20-week-old male Wistar-Kyoto rats (local inbred colony). The animals were killed by a sharp blow on the back of the head and exsanguination. The main pulmonary artery, thoracic aorta, and superior mesenteric artery, and the left carotid artery, renal and femoral artery as well as fourth-order sidebranches of the superior mesenteric artery were isolated under sterile conditions and cleaned of adhering fat.

\section{Preparation of tissues}

All arterial preparations were chemically sympathectomized with 6hydroxydopamine (Sigma Chemicals, St. Louis, Mo., USA) as described by Aprigliano and Hermsmeyer (1976). Segments of elastic and muscular arteries ( $2 \mathrm{~mm}$ long) were de-endothelialized by passing the shaft of an injection needle through their lumen (De Mey et al, $1991^{\mathrm{a}}$ ). These needles (outer diameter 0.7, 0.9 and $1.2 \mathrm{~mm}$ for femoral, renal and both carotid and mesenteric artery, respectively and $2.0 \mathrm{~mm}$ for pulmonary artery and thoracic aorta) obstructed the arterial lumen and did, as judged from Laplace's law, not distend the preparations beyond the level imposed by the transmural pressure in situ.

For experiments with mesenteric resistance-sized arteries, a small arterial tree, starting at the first-order branch of the mesenteric artery and continuing down-stream to a fourth-order branch, was carefully dissected but left attached to the gut. A fine stainless steel wire (diameter $0.04 \mathrm{~mm}$ ) was inserted into the largest branch and guided into the second-order branch. When the distal end of the fourth-order branch was cut, the tree retracted over the wire. As judged from histological examination, this did not result in excessive endothelium damage. To intentionally denude the endothelium from the small arteries, the thin wire was equipped with a single "old granny" knot (diameter 0.09-0.12 $\mathrm{mm})$.

In large and small arterial preparations that had not been intentionally denuded, $50-80 \%$ of the intimal surface was covered with endothelium. Intentional denudation appeared successful because it removed all intimal cells that were immunoreactive for von Willebrand factor and because it selectively abolished vasorelaxing responses to acetylcholine in all preparations (De Mey and Vanhoutte, $1982^{\mathrm{a}}$ ). It did not affect maximal contractile responses of the arterial segments to depolarization, suggesting that it was not accompanied by media damage. No regeneration of endothelium could be observed following organoid culture. Except when specifically mentioned, the data refer to observations in arterial preparations that had been intentionally denuded from endothelium. 


\section{Organoid culture}

To stimulate growth responses in the arterial preparations, the isolated segments ( $2 \mathrm{~mm}$ long) were suspended in $2.5 \mathrm{ml}$ medium. This consisted of Dulbecco's minimal Eagle's modified medium (DMEM; Gibco, Paisly, UK), containing $2 \mathrm{mM}$ glutamine (Gibco), $100 \mathrm{IU} / \mathrm{ml}$ penicillin (Gist-Brocades, Delft, The Netherlands) and $100 \mu \mathrm{g} / \mathrm{ml}$ streptomycin (Pharmachemie, Haarlem, The Netherlands). Except when specifically mentioned, the medium was supplemented with $20 \%$ fetal calf whole-blood serum (FCS; Seralab, Sussex, UK) that had been dialyzed (cut off, 6,000 Da) to remove small contractile components (dFCS)(De Mey et al, 1989). Qualitatively similar observations were obtained with different lots of FCS and with newborn calf and rat wholeblood serum. However, all data reported were, obtained in the presence of the same serum batch. Arterial preparations were maintained under these conditions at $37^{\circ} \mathrm{C}$ in an atmosphere of $95 \%$ air and $5 \% \mathrm{CO}_{2}$ at $97 \%$ humidity for 1-14 days. The arterial preparations were transferred to culture dishes filled with fresh medium after 1, 2, 3 and 6 days. To estimate the extent of DNA synthesis, $1 \mu \mathrm{M}$ 5-bromo-2'-deoxyuridine (BrdUrd, Sigma) was included in the medium. Two types of labeling protocol were used. The thymidine-analogue was either applied $24 \mathrm{~h}$ prior to fixation of the preparation or continuously during the first 4 culture days with daily replacement of the label. Preliminary observations indicated that BrdUrd was not degraded to a significant extent during these experiments.

Resistance arterial segments remained suspended on a fine stainless steel wire (diameter $0.04 \mathrm{~mm}$ ). Large arterial segments remained suspended on either a $0.25 \mathrm{~mm}$ wire (Dentaurum, Pforzheim, FRG) or on the shaft of the needle that was used to remove the endothelium. DNA synthesis did not differ significantly between de-endothelialized preparations that were mounted on a thin support and those suspended on the thicker denuding tool (Boonen et al, 1991). Cellular outgrowth was only quantified in the latter.

\section{Contractile reactivity}

Freshly isolated arterial segments and arterial segments that had been cultured were mounted horizontally in an organ chamber between an isometric force transducer (Kistler Morse DSC 6 or Statham UC2) and a displacement device. The organ chamber was filled with $25 \mathrm{ml} \mathrm{Krebs-Ringer} \mathrm{bicarbonate}$ solution (KRB) that was maintained at $37^{\circ} \mathrm{C}$ and aerated with $95 \% \mathrm{O}_{2}$ and $5 \%$ $\mathrm{CO}_{2}$ (De Mey and Vanhoutte, 1982 ${ }^{\mathrm{a}}$, De Mey et al, 1989). All preparations were placed at optimal diameter for mechanical performance by stepwise stretching and intermittent activation with high potassium solution. The KRB had the following composition (mmol/l): $\mathrm{NaCl}, 118.3 ; \mathrm{KCl}, 4.7 ; \mathrm{CaCl}_{2}, 2.5$; 
$\mathrm{MgSO}_{4} .7 \mathrm{H}_{2} \mathrm{O}, 1.2 ; \mathrm{KH}_{2} \mathrm{PO}_{4}, 1.2 ; \mathrm{NaHCO}_{3}, 25.0$, and glucose, 11.0. For high potassium-activating solution, all $\mathrm{NaCl}$ in $\mathrm{KRB}$ was replaced by an equimolar amount of $\mathrm{KCl}$. Contractile responses were expressed as increase in wall tension (increase in force/twice the segment length) and as increases in wall stress (increase in wall tension/mean media thickness).

\section{Cell culture}

ASMC of elastic, muscular and resistance arteries were obtained from explants (Chamley-Campbell et al, 1979) or during organoid culture of arterial segments. For explants of muscular arteries, segments were isolated and denuded from endothelium as described above. They were immersed in $200 \mu \mathrm{l}$ Hanks' balanced salt solution and cut into small pieces with sterile scissors. The pieces were then incubated in $1 \mathrm{ml}$ DMEM $+20 \%$ dFCS for 14 days to obtain isolated ASMC (Chamley-Campbell et al, 1979). ASMC could, however, also be obtained during organoid culture. We previously observed cells gather on the bottom of culture dishes in which a renal artery segment was suspended (Boonen et al, 1991). This was confirmed in the present study and was expanded to carotid, superior mesenteric and femoral artery segments. Also resistance arterial segments, which were not suited for the classical explant technique, gave rise to similar cells during organoid culture. Cells for experimentation were harvested during the 5th or 6th organoid culture day. After removal of the donor tissue from the culture well, the cells that had migrated out of the arterial segment continued to proliferate and could be subcultured. Subcultured cells from muscular arterial explants and those derived during organoid culture of muscular or resistance arterial segments were identified as smooth muscle cells because they (1) formed a hill and valley type of pattern in dense culture; (2) contained smooth muscle actin including smooth muscle alpha-actin, and because (3) their growth rate could be reduced by heparin in a growth-state-dependent fashion (Schwartz et al, 1986; Chamley-Campbell et al, 1979; Skalli et al, 1986).

To study growth kinetics of ASMC of different anatomical origin, cells were plated in 24-well plates (Greiner, Tubingen, GER) at a density of 5,000 cells $/ \mathrm{cm}^{2}$ in DMEM $+10 \% \mathrm{dFCS}$. The number of cells was determined daily in triplicate using a Burker-Turk hemocytometer. During the exponential growth phase, the slope of the linear regression line relating ${ }^{2} \log$ (cell number) to culture time was determined to estimate the growth rate (number of cell doublings per $24 \mathrm{~h}$ ). From this, the population-doubling time was determined. Growth kinetics did not differ between large-artery ASMC obtained by the classical explant technique and those derived during organoid culture and between primary cell cultures and up to the fifth passage. 


\section{Histology and immunohistochemistry}

Arterial segments were removed from organ culture after 1-14 days. They were rinsed for two $15 \mathrm{~min}$ periods in oxygenated Krebs-Ringer bicarbonate solution and fixed for $30 \mathrm{~min}$ in periodate-lysine-paraformaldehyde at $37^{\circ} \mathrm{C}$ (De Mey et al, 1989; Boonen et al, 1991). They were stored in phosphate-buffered saline at $4^{0} \mathrm{C}$ until they were embedded in paraffin. Crosssections ( $4 \mu \mathrm{m}$ thick) of large arteries and longitudinal sections of resistance arteries were stained with hematoxylin and eosin for general histological evaluation. Quantitative morphometry was performed on sections after ElastinVan Gieson staining using images generated by a video camera (Stemmer VS 450) and commercially available software (JAVA, Jandell, San Francisco, CA, USA).

For tissues that had been exposed to BrdUrd, we used a monoclonal antibody (Eurodiagnostics, Apeldoorn, The Netherlands) and an indirect enzyme-labeled antibody technique with a peroxidase-conjugated second step to visualize nuclei that had incorporated the thymidine analogue (Gratzner, 1982). To quantify the incorporation of BrdUrd, the percentage of nuclei that stained for peroxidase was counted in each arterial layer. Similar immunohistochemical techniques were used to evaluate the percentage of collagen IV (antibody 1024), smooth muscle alpha- and gamma-actin (antibody CGA 7), and smooth muscle alpha-actin (antibody SM-1) (Skalli et al, 1986).

\section{Statistics}

Data are shown as means \pm SEM. Statistical significance of differences was evaluated by one- or two-way analysis of variance where applicable. For multiple preplanned comparisons, the ANOVA was followed by Bonferroni's t-test (Wallenstein et al, 1980). $\mathrm{p}<0.05$ was taken to denote statistical significance of differences.

\section{RESULTS}

\section{Arterial segments without endothelium}

\section{Observations in carotid arteries}

In freshly isolated carotid artery segments, $125 \mathrm{mM}$ potassium induced an increase in wall tension averaging $2.91 \pm 0.13 \mathrm{mN} / \mathrm{mm}(\mathrm{n}=8)$. This mechanical response was reduced by $50 \%$ within $24 \mathrm{~h}$ of organoid culture and was little affected thereafter by up to 4 days of culture (fig. 4.1). 


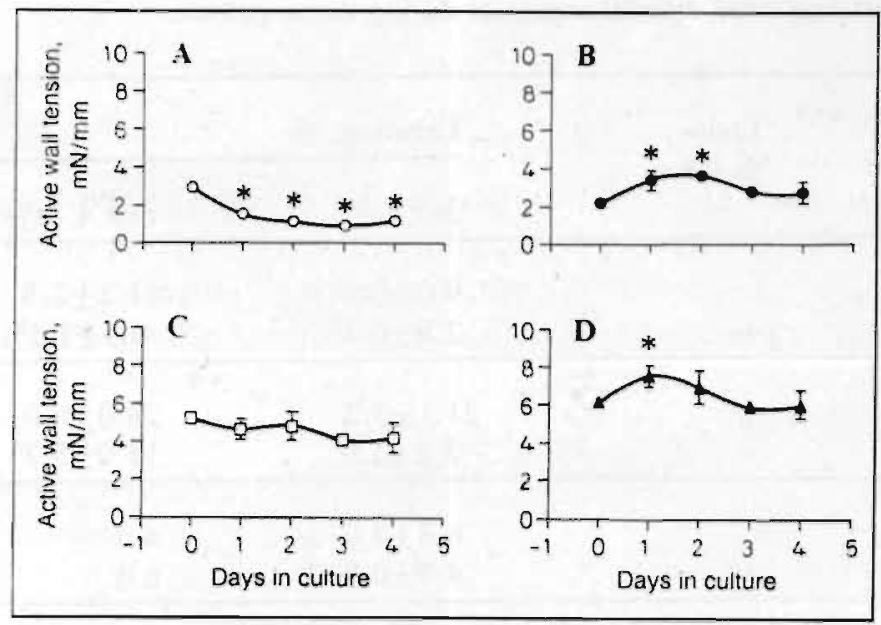

Figure 4.1 Effects of tissue culture on contractile reactivity of arterial segments. Segments of carotid (A), renal (B), superior mesenteric (C) and femoral (D) arteries of the rat were chemically sympathectomized, mechanically denuded of endothelium and maintained in tissue culture (see Materials and Methods section) in the continuous presence of $20 \% \mathrm{dFCS}$ for up to 4 days. The amplitude of their contractile responses to $125 \mathrm{mM}$ potassium at optimal lumen diameter was expressed as $\mathrm{mN} / \mathrm{mm}$ increase in wall tension. * $=$ Difference from observations in freshly isolated preparations is statistically significant.

During culture, nuclear incorporation of BrdUrd was stimulated (fig. 4.2). Following continuous exposure to BrdUrd during the initial 4 days of culture, $11.1 \pm 2.5 \%$ of the medial nuclei contained the thymidine analogue (table 4.1). Stimulation of DNA synthesis in the media was, however, transient. It was maximal during the third culture day, fell thereafter and was only minimal during the second week of culture (fig. 4.3). Also in serum-free medium some stimulation of intra-arterial DNA synthesis was observed (fig. 4.3). In the media, this reached only one fifth of the level observed in the presence of $20 \%$ serum. Yet, when compared to the absence of labeling during the first day and to the very low arterial DNA synthesis in adult animals in vivo (Schwartz et al, 1986) this could represent a marked, but only partial, stimulation in response to endogenous growth factors and trauma (Sjölund et al, 1988). Administration of serum to segments that had been maintained in serum-free conditions for 7 days, transiently stimulated DNA synthesis further. The time course and total extent of this response was comparable to that elicited by serum in freshly isolated preparations (fig. 4.3). 
Table 4.1 Effects of endothelium on nuclear incorporation of BrdUrd in the media of large and resistance-sized arterial segments during tissue culture.

\begin{tabular}{lccc}
\hline \multirow{2}{*}{$\begin{array}{l}\text { Artery } \\
\text { Endo- } \\
\text { thelium }\end{array}$} & Labeling, \% & \\
\cline { 3 - 4 } & & 3rd day & first 4 days \\
\hline Carotid & + & $9.0 \pm 0.5$ & $11.1 \pm 2.5$ \\
& - & $2.9 \pm 0.4^{\mathrm{a}}$ & $6.5 \pm 1.5^{\mathrm{a}}$ \\
\hline \multirow{2}{*}{ Renal } & + & $21.1 \pm 5.2$ & $28.0 \pm 6.0$ \\
& - & $7.2 \pm 1.9^{\mathrm{a}}$ & $18.0 \pm 5.7^{\mathrm{a}}$ \\
\hline \multirow{2}{*}{$\begin{array}{l}\text { Mesenteric } \\
\text { resitance }\end{array}$} & + & $6.8 \pm 2.0$ & n.d. \\
\hline
\end{tabular}

Arterial segments were maintained in culture for 3 or 4 days wit or without prior removal of endothelium. Tey were exposed to $1 \mu \mathrm{M}$ BrdUrd during either the third 24-hour period or continuously during the first 4 culture days. They were subsequently fixed and the percentage of labeled nuclei in the media was determined by immunohistochemistry. Data are shown as means $\pm \operatorname{SEM}(n=6-11)$. n.d. = not determined.

a The difference from observations in the absence of endothelium is statistically significant.

Up to 14 days of organoid culture did not increase the cross-sectional area of the media or the number of medial smooth muscle cells (table 4.2). It rather gave rise to new cell layers outside the original media and adventitia (fig. 4.2). In segments that had been mounted on obstructing supports, the outgrowth could only be observed at the adventitial side. In segments that had been suspended on a thin nonuccluding wire, new structures were formed at both the intimal and adventitial surface. The new structures that were present after 2 weeks contained elastin, collagen IV, smooth muscle myosin and smooth muscle actin, including smooth muscle alpha-actin (not shown). After 2 weeks, the new cell layers contained $662 \pm 66(n=11)$ nuclear profiles per cross-section and thus outnumbered the media ( $314 \pm 14$ profiles per crosssection). While DNA synthesis had ceased in the media after 14 days of culture, nuclear incorporation of BrdUrd was still present in the newly formed smooth muscle-like structure at this point in time (fig. 4.2). For the whole layer it averaged $13.8 \pm 2.7 \%$ per $24 \mathrm{~h}(\mathrm{n}=11)$. It was, however, virtually limited to the outermost cell layer (fig. 4.2).

During organoid culture of suspended carotid artery segments, small numbers of cells migrated out of the tissue and could be recovered on the bottom of the culture wells. These cells, which could be subcultured, shared 


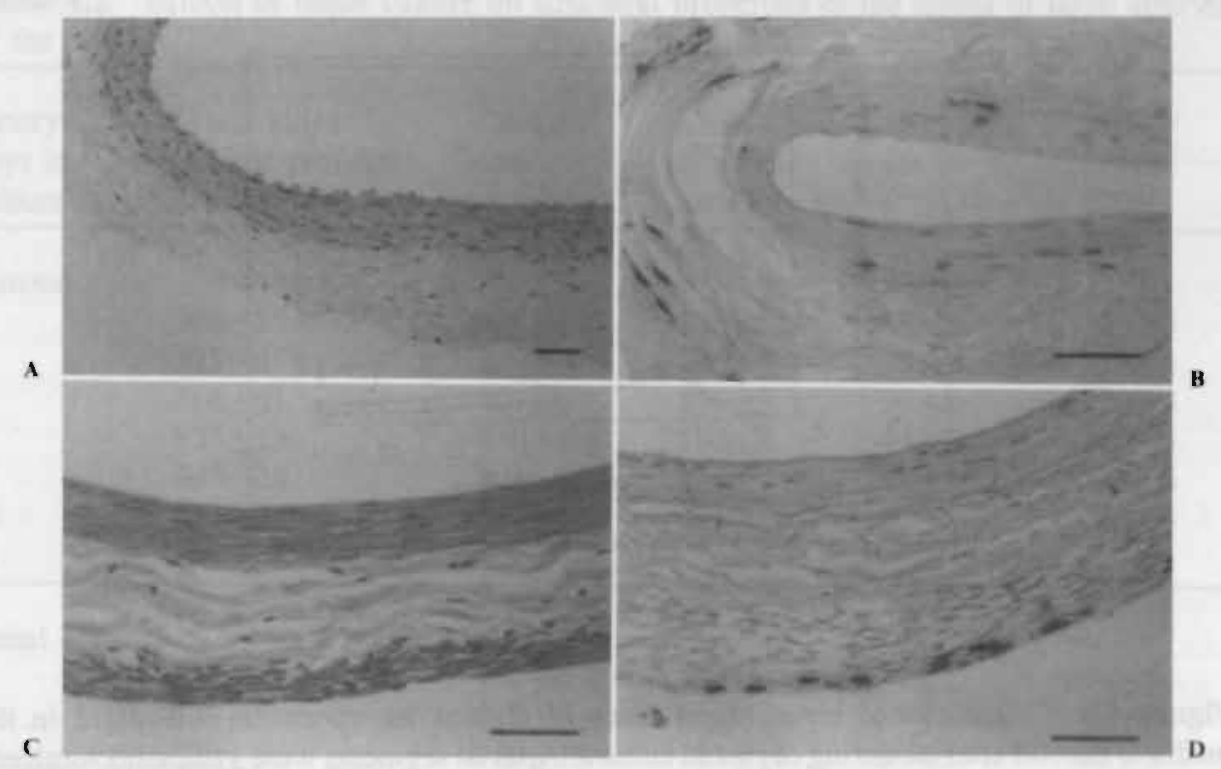

Figure 4.2 Light micrographs of cross-sections of the carotid artery of the rat. A Freshly isolated preparation stained with hematoxylin and eosin. B De-endothelialized preparation maintained in culture for 4 days in the continuous presence of $20 \% \mathrm{dFCS}$ and $1 \mu \mathrm{M}$ BrdUrd. The vessel was fixed on the myograph following recording of active wall tension. The section was stained immunohistochemically for BrdUrd and counterstained with hematoxylin. C, D De-endothelialized preparation maintained in culture for 14 days in the continuous presence of $20 \%$ dFCS exposed to $1 \mu \mathrm{M}$ BrdUrd during the last $24 \mathrm{~h}$. Sections were stained with hematoxylin and eosin $(\mathbf{C})$ or immunohistochemically for BrdUrd and counterstained with hematoxylin (D). Bars indicate $50 \mu \mathrm{m}$.

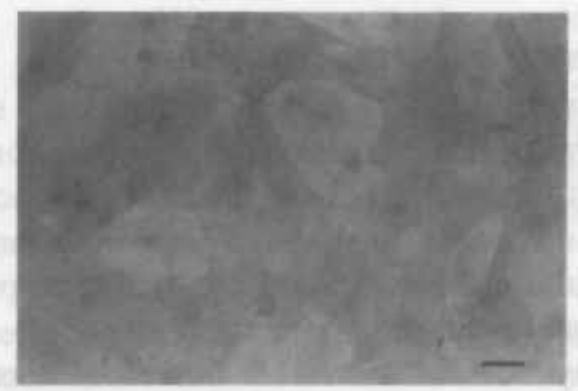

Figure 4.6 Photomicrograph of mesenteric resistance-artery-derived cells grown in vitro. The cells were stained immunohistochemically for smooth muscle alpha-actin (antibody SM1) and counterstained with hematoxylin. The bar indicates $50 \mu \mathrm{m}$. 


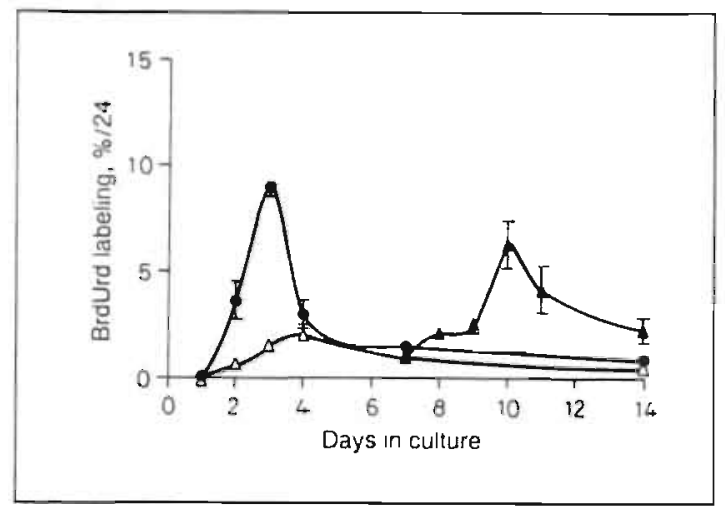

Figure 4.3 Kinetics and serum dependence of nuclear incorporation of BrdUrd in the media of carotid arteries during organoid culture. Arterial segments were sympathectomized, denuded of endothelium and maintained in tissue culture in the absence $(\Delta)$ or presence $(0, \Delta)$ of $20 \%$ dFCS. Note that some of the preparations (A) were exposed to serum after having been maintained in serum-free conditions for 7 days. $1 \mu \mathrm{M}$ BrdUrd was included in the culture medium during the last $24 \mathrm{~h}$ of culture. After 1-14 days of culture, the segments were fixed and the percentage of medial nuclei that had incorporated the thymidine analogue was determined by immunohistochemistry. The data are shown as means \pm SEM $(n=6)$.

structural, immunohistochemical and pharmacological properties with carotid artery smooth muscle cells prepared by the conventional explant method. Their growth kinetics at low density (population doubling time, $0.99 \pm 0.3$ days) did not differ from that of explant cells $(0.95 \pm 0.3$ days $)$.

\section{Comparison between muscular arteries}

Contractile responses to $125 \mathrm{mM}$ potassium were significantly larger in freshly isolated femoral and mesenteric artery preparations than in carotid and renal artery segments (fig. 4.1). This applied for both active wall tension and active wall stress. Unlike described above for carotid artery segments, up to 4 days of organoid culture did not reduce contractile responses to potassium in femoral, mesenteric and renal artery segments (fig. 4.1). In femoral and renal preparations, 1-2 days of culture rather increased mechanical response to depolarization (fig. 4.1).

Qualitatively similar growth responses to organoid culture were observed in arterial segments of different anatomical origin. In 4 types of artery the growth responses consisted of (1) transient stimulation of DNA synthesis in 
Table 4.2 Effects of tissue culture on structural properties of the media of large arteries of the rat ${ }^{\mathrm{a}}$.

\begin{tabular}{llllll}
\hline $\begin{array}{l}\text { Artery } \\
\text { days in } \\
\text { culture }\end{array}$ & $\begin{array}{l}\text { Plasia index } \\
\text { nuclear profiles } \\
\text { cross-section }\end{array}$ & $\begin{array}{l}\text { Diameter } \\
\mu \mathrm{m}\end{array}$ & $\begin{array}{l}\text { Cross- } \\
\text { sectional } \\
\text { area } \mu \mathrm{mx} 10^{-3}\end{array}$ & $\begin{array}{l}\text { Thickness } \\
\mu \mathrm{m}\end{array}$ & $\begin{array}{l}\text { Media/ } \\
\text { lumen } \\
\times 10^{3}\end{array}$ \\
\hline
\end{tabular}

\section{Carotid}

$\begin{array}{llllll}0 & 309 \pm 25 & 800 \pm 10 & 139 \pm 14 & 51 \pm 3 & 128 \pm 5 \\ 1 & 323 \pm 63 & 809 \pm 3 & 135 \pm 5 & 50 \pm 2 & 120 \pm 4 \\ 2 & 314 \pm 61 & 809 \pm 9 & 136 \pm 3 & 50 \pm 3 & 120 \pm 6 \\ 3 & 331 \pm 13 & 810 \pm 6 & 132 \pm 8 & 49 \pm 2 & 120 \pm 5 \\ 4 & 346 \pm 23 & 804 \pm 4 & 125 \pm 7 & 47 \pm 2 & 120 \pm 5 \\ 14 & 314 \pm 21 & 814 \pm 17 & 107 \pm 1 & 46 \pm 8 & 140 \pm 5\end{array}$

\section{Renal}

\begin{tabular}{llllll}
0 & $187 \pm 11$ & $780 \pm 4$ & $96 \pm 5$ & $37 \pm 1$ & $96 \pm 3$ \\
1 & $149 \pm 31$ & $727 \pm 6$ & $86 \pm 8$ & $36 \pm 3$ & $97 \pm 6$ \\
2 & $222 \pm 56$ & $730 \pm 18$ & $103 \pm 16$ & $39 \pm 5$ & $98 \pm 8$ \\
3 & $166 \pm 8$ & $714 \pm 16$ & $79 \pm 5$ & $34 \pm 1$ & $94 \pm 3$ \\
4 & $168 \pm 40$ & $734 \pm 22$ & $80 \pm 18$ & $33 \pm 5$ & $88 \pm 8$ \\
14 & $174 \pm 35$ & $784 \pm 8$ & $96 \pm 15$ & $37 \pm 5$ & $91 \pm 11$ \\
\hline
\end{tabular}

Superior mesenteric

\begin{tabular}{llllll}
0 & $378 \pm 24$ & $952 \pm 13$ & $206 \pm 13$ & $65 \pm 3$ & $135 \pm 5$ \\
1 & $335 \pm 16$ & $906 \pm 10$ & $165 \pm 10$ & $54 \pm 3$ & $120 \pm 5$ \\
2 & $319 \pm 38$ & $806 \pm 49$ & $156 \pm 10$ & $55 \pm 2$ & $129 \pm 2$ \\
3 & $450 \pm 37$ & $744 \pm 55$ & $176 \pm 31$ & $59 \pm 5$ & $141 \pm 13$ \\
4 & $363 \pm 17$ & $636 \pm 56$ & $188 \pm 18$ & $62 \pm 5$ & $137 \pm 9$ \\
14 & $326 \pm 25$ & $792 \pm 55$ & $147 \pm 24$ & $58 \pm 5$ & $107 \pm 4$ \\
\hline & & & & \\
Femoral & $237 \pm 13$ & $650 \pm 4$ & $123 \pm 7$ & $56 \pm 3$ & $172 \pm 11$ \\
0 & $246 \pm 22$ & $648 \pm 3$ & $124 \pm 11$ & $55 \pm 4$ & $169 \pm 10$ \\
1 & $207 \pm 8$ & $646 \pm 4$ & $104 \pm 5$ & $47 \pm 2$ & $142 \pm 8$ \\
2 & $242 \pm 16$ & $654 \pm 5$ & $116 \pm 10$ & $51 \pm 4$ & $153 \pm 11$ \\
3 & $232 \pm 14$ & $628 \pm 8$ & $101 \pm 6$ & $48 \pm 5$ & $155 \pm 25$ \\
4 & $340 \pm 25$ & $650 \pm 2$ & $103 \pm 2$ & $48 \pm 1$ & $150 \pm 10$ \\
14 & & & & & \\
\hline
\end{tabular}

a Data are shown as means \pm SEM $(n=5-12)$ for arterial segments that had been sympathectomized, mechanically denuded of endothelium and maintained in tissue culture in the presence of $20 \%$ dialyzed fetal calf serum. Plasia was estimated by counting the number of nuclear profiles in the media of cross-sections stained with hematoxylin-eosin. Lumen diameter and cross-sectional area were determined by morphometry on cross-sections stained with Elastin-Van Gieson. Mean media thickness and wall-to-lumen ratio were derived from the diameter and area measurements. 


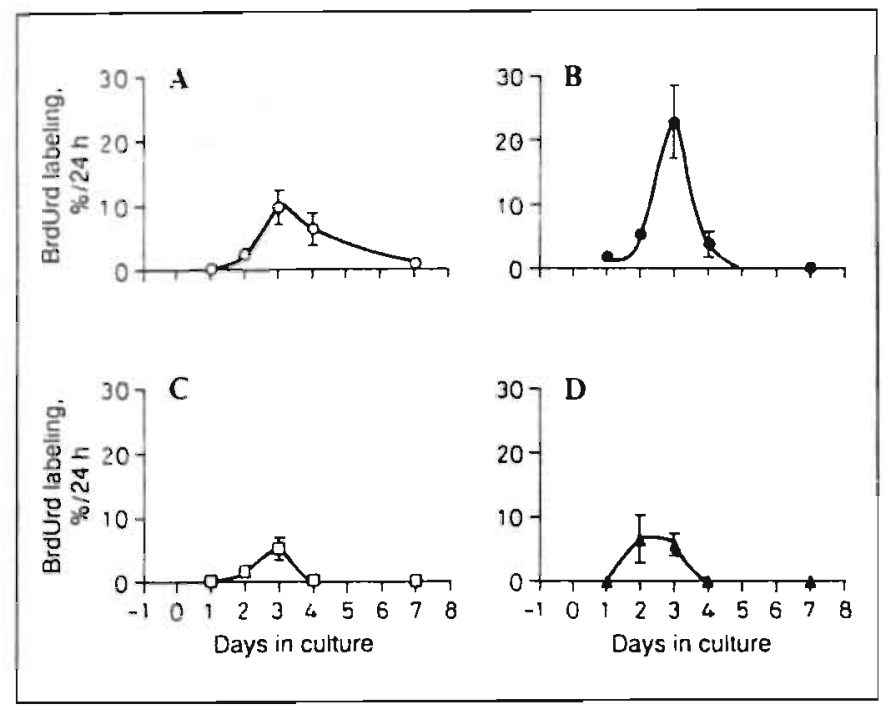

Figure 4.4 Nuclear incorporation of BrdUrd in the media of large arteries during tissue culture. Segments of carotid (A), renal (B), superior mesenteric (C) and femoral arteries (D) were chemically sympathectomized, mechanically denuded of endothelium and maintained in tissue culture in the presence of $20 \% \mathrm{dFCS}$. $1 \mu \mathrm{M}$ BrdUrd was included in the medium during the last $24 \mathrm{~h}$ of culture. After $1,2,3,4$, or 14 days of culture, the segments were fixed and the nuclear labeling was evaluated by immunohistochemistry on cross-sections. The data were expressed as the percentage of medial nuclei that were labeled during the 24 hour period. The data are shown as means \pm SEM $(n=5-8)$.

the arterial media, (2) formation of smooth muscle-like structures outside the original media and adventitia and (3) escape of smooth muscle-like cells from the arterial segments and their proliferation outside the tissue.

Despite this qualitative similarity, the extent of DNA synthesis in the media differed markedly between the types of artery (fig. 4.4). In renal artery preparations, the maximal daily DNA synthesis was on a relative basis $2-3$ times higher than in the carotid artery. In mesenteric arteries, it was significantly smaller than in carotid artery segments. Also the total intramedial nuclear incorporation of BrdUrd that was accumulated during 4 days of continuous exposure to the thymidine analogue was significantly larger in renal than carotid arteries (table 4.1). In contrast, incorporation of BrdUrd in adventitial fibroblasts which in all arteries was significantly larger than in smooth muscle cells, did not differ between the 4 types of artery (data not 
shown).

In none of the types of artery even 14 days of organoid culture resulted in media hypertrophy or media hyperplasia (table 4.2). During the 2-week period, a marked hyperplastic response developed, however, outside the original media and adventitia. As judged from the number of nuclear profiles/cross-section, the size of the new layer tended to be larger in renal $(797 \pm 138 ; n=8)$ and femoral arteries $(753 \pm 75 ; n=7)$ than in carotid $(662$ $\pm 66 ; n=11)$ and superior mesenteric arteries $(551 \pm 84 ; n=8)$. Since immunohistochemical observations suggest that the newly formed layers originated from smooth muscle cells that migrated out of the arterial media, we normalized the size of the new layer to the number of smooth muscle cells in the media of freshly isolated arteries. This ratio was significantly larger for renal $(4.25 \pm 0.26)$ and femoral arteries $(3.18 \pm 0.19)$ than for carotid $(2.11$ $\pm 0.09)$ and mesenteric arteries $(1.46 \pm 0.13)$.

In order to discriminate between factors endogenous to the ASMC and factors in the environment of the cells as causes for regional differences of in vitro growth responses, we recorded the rate of proliferation of smooth muscle Arterial segments with endotheliumcells isolated from the 4 types of artery. Population doubling times of cells seeded at low density were, however, comparable for carotid $(0.99 \pm 0.03$ days $)$, mesenteric (1.05 \pm 0.02 days), renal ( $1.09 \pm 0.01$ days) and femoral arteries (0.99 \pm 0.09 days).

Alternatively, the different growth responses could have resulted from differences in the structural organization of the arteries. It is of interest in this respect that the media of renal arteries, which responded most dramatically to tissue culture, was considerably thinner and contained less smooth muscle cells than that of the other types of artery (table 4.2).

\section{Elastic and resistance-sized arteries}

In the media of isolated thoracic aorta and main pulmonary artery, which have a thick media but a small wall to lumen ratio, nuclear incorporation of BrdUrd was low. During the 3rd day of organoid culture, it averaged $6.5 \pm$ 1.6 and $2.6 \pm 0.5 \%$, respectively $(n=6)$. These values are comparable to those observed in femoral and mesenteric arteries and are significantly smaller than the observations in renal artery segments.

Also in mesenteric resistance arteries, which display a thin media (15 土 $2 \mu \mathrm{m})$ but a high wall-to-lumen ratio $(0.183 \pm 0.028)$, intramedial DNA synthesis during culture was low. It averaged $6.8 \pm 2.1 \%(n=8)$ during the third 24-hour period. Also this value is comparable to those observed in femoral and superior mesenteric artery. 


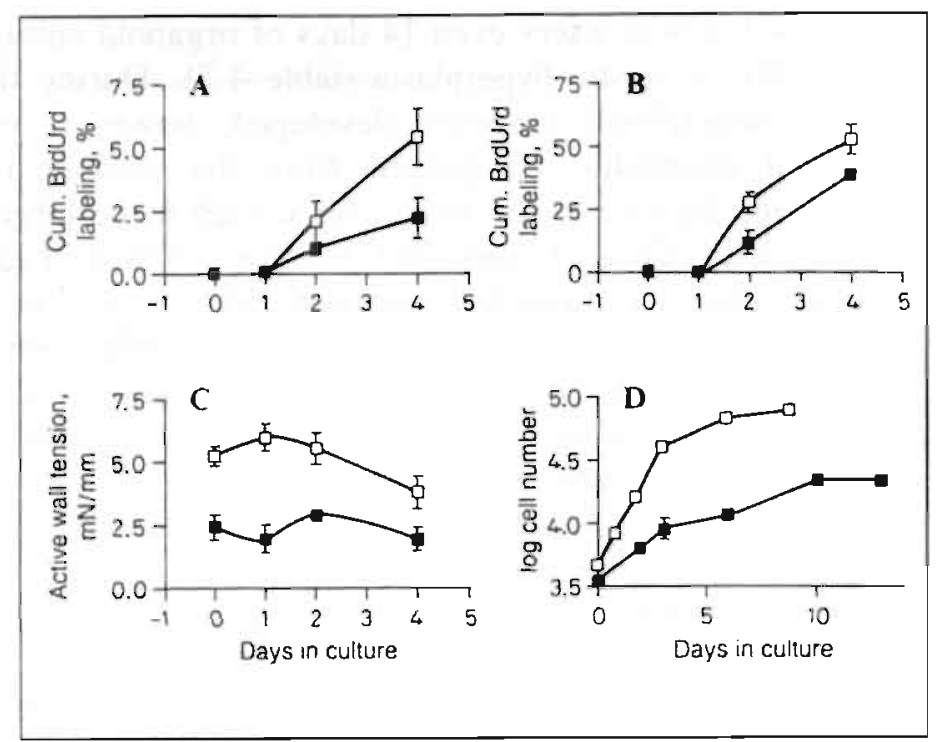

Figure 4.5 Effects of culture on segments and cells of mesenteric resistance arteries ( $\square)$ and superior mesenteric arteries ( $\square$ ). The segments had been chemically sympathectomized and were maintained in the continuous presence of $20 \% \mathrm{dFCS}$ and $1 \mu \mathrm{M}$ BrdUrd. The graphs show the relative extent of nuclear BrdUrd labeling in the media (A) and adventitia (B) and the amplitude of contractile responses to $125 \mathrm{mM}$ potassium at optimal diameter (C) after up to 4 days of culture. The data are shown as means \pm SEM $(n=5-9)$. D. Proliferation of smooth muscle-like cells during cell culture. The initial densities of cells derived from large ( $\square$ ) and small ( $\square$ ) mesenteric arteries were comparable.

\section{Arterial segments with endothelium}

In the media of both carotid and renal artery segments with endothelium, significantly less DNA synthesis was observed following culture than in originally adjacent segments that had been denuded of endothelium (table 4.1). This applied not only for the nuclear incorporation of BrdUrd during the third culture day but also, albeit to a lesser extent, for the cumulative incorporation of thymidine analogue during the initial 4 days of culture (table 4.1).

In mesenteric blood vessels, modulation of medial growth responses by the endothelium was less obvious. No clear-cut difference could be observed between superior mesenteric artery segments with and without endothelium. Also in resistance-sized side-branches of the mesenteric artery, mechanical removal of the endothelium did not significantly affect DNA synthesis in the 
media during culture (table 4.1).

A variable degree of subtle media damage could accompany mechanical removal of the endothelium in large and small arteries. Since the endothelium did not significantly affect growth responses in mesenteric vessels, we performed an additional comparative study in superior mesenteric and mesenteric resistance arterial segments that had not been denuded from endothelium. To evaluate whether arterial growth responsiveness changes with branching, we used continuous exposure to BrdUrd to cumulate the suspected low extent of intramedial DNA synthesis (see above). The data are summarized in figure 4.5. While nuclear incorporation in the adventitial fibroblasts did not differ between large and small vessels, BrdUrd labeling in the media was also significantly larger, on relative basis, in superior mesenteric arteries than in mesenteric resistance arteries.

Growth responses did not only differ between the media of the large and small vessels, but also between isolated smooth muscle cells of the muscular and resistance-sized arteries (fig. 4.5). The rate of proliferation of resistanceartery-derived cells (population doubling time: $4.07 \pm 0.65$ days, $n=9$ ) was 4 times slower than that of large artery cells (fig. 4.5). This difference persisted after at least 5 passages. Yet, the resistance arterial cells, like large artery cells, contained "stress" fibers composed of smooth muscle alpha-actin (fig. 4.6). Note that at low density not all resistance-artery-derived cells displayed SM1-immunoreactivity. Both cells containing and lacking smooth muscle alpha-actin can be observed (fig. 4.6). At high density, however, all cells stained for smooth muscle alpha-actin (not shown). This could be indicative for a density and/or growth state dependence of the expression of this contractile protein (Schwartz et al, 1986; Chamley-Campbell et al, 1981; Blank et al, 1988).

\section{DISCUSSION}

In a broad variety of arterial preparations, organ culture stimulated DNA synthesis in the arterial media and induced proliferation of smooth-muscle-like cells. The amplitude of these responses differed between arterial segments of different anatomical origin and branching order.

Culture induced but a transient stimulation of DNA synthesis in the media of isolated arterial segments. A similar transient response was previously observed in carotid arteries following mechanical injury in situ (Clowes et al, 1989). In vitro, a small but significant stimulation could also be observed in the absence of serum. This confirms the possibility of autocrine growth stimulation in the arterial wall (Walker et al, 1986; Sjölund et al, 1988; Majesky et al, 1988) but indicates that the major part of the DNA response in the presence of 
serum can be attributed to exogenous factors. In preliminary experiments (Uitendaal and De Mey, unpubl. obs.), similar stimulation of intramedial DNA synthesis was observed in the presence of 2 and $20 \%$ serum. This, together with the regular replacement of the culture media, suggests that a high level of exogenous mitogens was maintained throughout the entire culture period. Yet, the DNA response was highly transient. This response could be delayed by serum-free conditions and did not correlate with changes in contractility. It is therefore unlikely that the transient nature of the response was due to decreased viability of the tissue. Stimulation of DNA synthesis was not only transient but it was also restricted to a fraction of the medial cells as is clear from the continuous labeling experiments (table 4.1). Serum growth factors thus could stimulate DNA synthesis in only part of the arterial media cells.

In the arterial media of young rats, the distribution of desmin, alpha-actin and myosin is not uniform (Skalli et al, 1986), suggesting the presence of a mixture of arterial smooth muscle cell subtypes. On the basis of the (1) expression of platelet-derived growth factor genes and release of plateletderived growth-factor-like molecules (Majesky et al, 1988), and (2) the appearance of polyploidy of ASMC in vivo and in vitro (Barrett et al, 1983; Gordon et al, 1986), it has been suggested that these subsets of ASMC could have different growth characteristics.

Our observations tend to suggest that in the large arterial wall of adult rats, despite uniform distribution of contractile phenotype markers (De Mey and Fazzi, unpubl. obs.), a small fraction of the smooth muscle cells remained in a growth-prone state. These could participate in responses to injury (Schwartz et al, 1986). In view of the observed transient nature of intramedial DNA synthesis, the responsiveness of these cells to mitogens would not be shared by their daughters, provided that these remain in the arterial media. Culture did, however, not increase the number of medial cells. It rather resulted in proliferation of cells outside the media. These new cells were, at least partly, smooth-muscle-like cells since they displayed a number of properties that in freshly isolated arteries were restricted to the media. Originally, quiescent cells or daughter cells of growth-prone cells thus were attracted out of the media and proliferated at the edge of the tissue.

In all types of arteries, qualitatively similar responses to organoid culture were observed. Despite attempts to study all preparations under identical external conditions, the responses differed quantitatively between arteries of different anatomical origin. Whether this was due to regional differences in smooth muscle function or in the structure of the vessels remained largely unclear. In renal arteries, intramedial DNA synthesis was more pronounced than in other types of artery. Yet, smooth muscle cells isolated from renal arteries proliferated in cell culture at a rate that did not differ from that of smooth muscle cells from other large arteries. This and the transient nature of 
DNA synthesis indicate that phenomena within the arterial media modulated the growth responsiveness of the arterial smooth muscle cells. Aspects such as cellular phenotype, cell density and extracellular matrix components could be involved (Schwartz et al, 1986; Chamley-Campbell et al, 1981; Blank et al, 1988). But also factors, other than the overall organization of the arterial media, may have contributed to the observed regional variability of growth responses in vitro. In view of the possible presence of subsets of arterial smooth muscle cells that differ in terms of their growth kinetics (Schwartz et al, 1986; Majesky et al, 1988; Barrett et al, 1983; Gordon et al, 1986 and above), it is not unlikely that isolation and subculture of cells selected growthsensitive cells (Schwartz et al, 1986). These did not differ between types of large artery but their original density might have differed between arteries of different anatomical origin. Confirmation of this possibility will depend on the development of workable markers that dissociate between the subtypes of arterial smooth muscle cells.

Not only in renal arteries but also in mesenteric resistance-sized arteries the observations deviated from those in carotid, superior mesenteric and femoral artery. In the resistance arteries the maximal daily intramedial DNA synthesis was in the order of what was to be expected on the basis of most types of large arteries. After continuous exposure to BrdUrd, however, significantly less labeling was observed in the muscle of resistance arteries than in that of superior mesenteric arteries. Differences following such a long-term approach can be indicative of differences not only in DNA synthesis but also in the frequency of nuclear division. Cells that had migrated out of resistance arterial segments proliferated considerably slower than large arterial cells. This difference persisted for many cellular generations in vitro. Yet, on the basis of their smooth muscle alpha-actin content, both the large and the small arterial cells have to be considered as smooth muscle cells. This finding strengthens the possibility that heterogeneous populations of contractile arterial smooth muscle cells exist which differ in terms of their growth characteristics. It is of interest that while medial cross-sectional area was 20 times smaller in mesenteric resistance arteries $\left(8 \pm 1 \mu \mathrm{m}^{2} \cdot 10^{-3}, \mathrm{n}=8\right)$ than in the superior mesenteric artery (table 2), active wall tension in the small vessels was half that in the large vessels. This raises the possibility that smooth muscle cells of small arteries might exist in a more contractile phenotype and hence be less likely to proliferate.

In carotid and renal arteries the presence of endothelium significantly reduced medial DNA synthesis during organoid culture. The effect of the endothelium was more marked during the 3rd culture day (De Mey et al, $1991^{a}$ ) than during the entire initial 4 day period. This could indicate an effect on the kinetics rather than on the maximal extent of DNA synthesis. In resistance-sized mesenteric arteries, however, removal of endothelium did not 
vignificantly affect medial DNA synthesis. In previous studies in which conditioned media, endothelial and smooth muscle cell coculture, and organoid culture were used, the endothelium was observed to stimulate, to inhibit and not to affect arterial smooth muscle cell proliferation (Davies, 1986; StaianoCoico et al, 1988; De Mey et al, 1991 ${ }^{\mathrm{a}}$; Koo and Gotlieb, 1989). These discrepancies have been attributed to the potential of endothelial cells to generate both stimulatory and inhibitory growth signals depending on their growth state (Davies, 1986; Koo and Gotlieb, 1989). An alternative and complementary possibility could be that as for smooth muscle, the endothelium is not uniform throughout the vascular system. This was previously documented for the production of endothelium-derived vasoactive and antithrombotic agents (Davies, 1986; De Mey and Vanhoutte, 1982 ${ }^{\mathrm{a}}$; Gryglewsky et al, 1988).

In summary, we observed differences in growth responses to supramaximal concentrations of exogenous growth factors in isolated arterial segments of different anatomical origin and branching order. We speculate that this could be due to the heterogeneous distribution over the arterial tree of different subsets of arterial smooth muscle cells. This, together with possible differences in modulatory influences of the endothelium on arterial smooth muscle growth, indicates that there is no single cellular preparation that can be used as a valid model system for intra-arterial growth control in general. This should be taken into account when attempting to extrapolate findings in for instance aortic smooth muscle cell culture to evaluated vascular resistance in hypertension. Heterogeneity of local intra-arterial growth control could, on the other hand, contribute to regional differences in susceptibility to structural alteration and in type of structural changes that occur during the pathogenesis of vascular diseases. 


\section{Chapter}

\section{DNA Synthesis in Isolated Arteries of Normotensive and Hypertensive Rats: Effects of the Endothelium}

P.M.H. Schiffers, G.E. Fazzi, G.M.J. Janssen, M.P. Uitendaal, H.A.J. Struijker-Boudier and J.G.R. De Mey

Department of Pharmacology, University of Limburg, Maastricht, the Netherlands. (Journal of Hypertension, submitted) 


\section{INTRODUCTION}

In established hypertension the wall:Iumen ratio is increased in large and small arteries (Folkow, 1982; Mulvany et al, 1978). This can be reversed by some but not all forms of antihypertensive therapy (Struijker-Boudier et al, 1990). Cells isolated from the thoracic aorta of spontaneously hypertensive rats, have repeatedly been observed to proliferate faster than those of normotensive rats (Hadrava et al, 1989; Resink et al, 1987). It has therefore been proposed that abnormal growth control in arterial smooth muscle cells could participate in the pathogenesis of essential hypertension. The type of arterial structural change in animal models of hypertension differs, however, between different parts of the arterial system (Struijker-Boudier et al, 1990). Depending on the anatomical location it may include cellular hypertrophy, hyperplasia and even structural narrowing without a change in muscle mass (Mulvany et al, 1978; Schwartz et al, 1986; Owens, 1989; Mulvany et al, 1985; Owens et al, 1988; De Mey et al, 1991 ${ }^{\text {b }}$ ). Furthermore, growth responses in the arterial wall do not only depend on cellular properties. They are also influenced by externally supplied trophic stimuli and growth inhibitory influences (Thyberg et al, 1990; Resink et al, 1989). Besides nerves (Head, 1991; Donohue et al, 1989) and extracellular matrix components (Thyberg et al, 1990) also the endothelium (Davies, 1986; DiCorletto et al, 1983; Warner et al, 1987; Castellot et al, 1981; De Mey et al, 1991 ${ }^{\mathrm{a}}, 1991^{\mathrm{c}}$; Koo and Gotlieb, 1989; Joseph-Silverstein and Rifkin, 1987) could be involved herein.

The endothelium can release a variety of substances that affect arterial tone (Furchgott and Vanhoutte, 1989; Vanhoutte, 1989; Gryglewski et al, 1988). Both relaxing and contractile endothelium-derived-factors have been identified (Furchgott and Vanhoutte, 1989; Vanhoutte, 1989; Gryglewsky et al, 1988; Miller and Vanhoutte, 1988; Palmer et al, 1987; Yanagisawa et al, 1988). In established hypertension the balance between these is altered (Furchgott and Vanhoutte, 1989; De Mey and Gray, 1985; Lüscher, 1990). The endothelium may also interfere with growth responses in the underlaying arterial smooth muscle. In culture media conditioned by endothelial cells, mediators have been found that can either promote (DiCorletto et al, 1983; Warner et al, 1987; Yanagisawa et al, 1988) or inhibit (Davies, 1986; Castellot et al, 1981) proliferation of isolated arterial smooth muscle cells.

In the present study we evaluated whether (i) the endothelium influences growth responses in the arterial wall and whether (ii) hypertension alters this process. For this purpose we used organ culture of isolated arterial segments (De Mey et al, 1991 ${ }^{\mathrm{a}}, 1991^{\mathrm{c}}, 1989$; Boonen et al, 1991) of normotensive Wistar (WIS), Wistar-Kyoto (WKY) and Sprague-Dawley (SDR) rats and of spontaneously hypertensive (SHR) rats and transgenic SDR harbouring the mouse Ren-2 gene (TGR, Mullins et al, 1990). To dissociate primary 
interstrain differences from secondary adaptive changes to elevated blood pressure, the experiments were performed in vessels of 6- and 20 -week old rats and in 20-week old WKY that had been made hypertensive by partial aorta coarctation. Because growth responses differ, at least quantitatively, between arterial segments of different anatomical origin (De Mey et al, 1991 ${ }^{c}$ ), experiments were performed in carotid and renal artery segments.

\section{MATERIALS AND METHODS}

Experiments were performed with male WKY and SHR (local inbred strains; Rijksuniversiteit Limburg, Maastricht, The Netherlands), male WIS (Charles River, FRG) and with male SDR and TGR rats (kindly supplied by Dr. J.J. Mullins, AFRC Centre for Genome Research, University of Edinburgh, Edinburgh, UK). At 6 weeks of age, systolic blood pressure, recorded by the tail cuff method, did not differ significantly between WKY $(89 \pm 4$ $\mathrm{mmHg})$ and SHR $(100 \pm 5 \mathrm{mmHg})$. At 20 weeks, systolic blood pressure averaged $136 \pm 3$ and $198 \pm 4 \mathrm{mmHg}(\mathrm{p}<0.05)$ in WKY and SHR, respectively. Mean arterial pressure (recorded by intra-arterial catheter) in 11 week old TGR and age-matched SDR, averaged $222 \pm 17$ and $119 \pm 1 \mathrm{mmHg}(\mathrm{p}<0.05)$, respectively.

In a separate group of WKY $(n=8)$, aorta coarctation was induced at 17 weeks of age. Under ether anesthesia and sterile conditions, the abdomen was opened and the abdominal aorta was tied between the superior mesenteric artery and the left renal artery onto the shaft of a stainless steel rod (diameter $0.7 \mathrm{~mm}$ ). This tool was subsequently removed, leaving the abdominal aorta partially obstructed. The abdomen was closed and the animals were allowed to recover. Three weeks later (20 weeks of age) mean arterial blood pressure measured under ether anesthesia through a carotid and a femoral artery catheter, averaged $139 \pm 6$ and $98 \pm 11 \mathrm{mmHg}$, respectively. Carotid and femoral artery pressure in corresponding shams averaged $111 \pm 5$ and $108 \pm 4$ $\mathrm{mmHg}$, respectively.

From 6 week old SHR and WKY, 20-week old SHR, WIS, WKY and aorta-coarcted WKY, and from 11 week old TGR and SDR, carotid and renal arteries were isolated under sterile conditions. In previous experiments no significant differences were observed between left and right carotid or renal arteries of WKY (De Mey et al, 1991 a 1991 ${ }^{\mathrm{c}}$; Boonen et al, 1991). In this study, arterial segments were randomly obtained from the left and right side of SHR, TGR, WIS, SDR and normotensive WKY. Separate experiments were, however, performed with the right and left renal arteries that were obtained from the upper and lower body parts of aorta-coarcted WKY.

All isolated arteries were chemically sympathectomized (De Mey et al, 
$1991^{\mathrm{a}}, 1991^{\mathrm{c}}, 1989$; Boonen et al, 1991) by a $10 \mathrm{~min}$ incubation $\left(37^{\circ} \mathrm{C}\right)$ in bicarbonate-free Krebs Ringer solution containing $300 \mu \mathrm{g} / \mathrm{ml}$ 6-hydroxydopamine (Sigma Chemicals, Saint Louis, Mo, USA). Following a subsequent 20 min incubation in drug-free Krebs-Ringer bicarbonate solution aerated with $95 \% \mathrm{O}_{2}$ and $5 \% \mathrm{CO}_{2}$, two segments ( $2 \mathrm{~mm}$ wide) were prepared from each artery. Of these pairs one segment was gently pulled over the shaft of an hypodermic injection needle (outer diameter 0.8 and $0.7 \mathrm{~mm}$ for carotid and renal arteries, respectively). This removed the cellular lining of the lumen that was immunoreactive for von Willebrand factor and angiotensin-converting enzyme and irreversibly but selectively abolished arterial relaxing responses to acetylcholine and histamine. This procedure will be referred to as endothelium removal. It did previously not reduce maximal contractile responses to depolarizing solution or serotonin and did not markedly affect the DNA content of the arterial preparations, suggesting that it was not accompanied by medial injury (De Mey et al, 1991 ${ }^{\mathrm{a}}, 1991^{\mathrm{c}}$; Clowes et al, 1989).

Arterial organ culture was performed as previously described (De Mey et al, 1991 a $1991^{\mathrm{c}}, 1989$; Boonen et al, 1991). Arterial segments were mounted on a $0.25 \mathrm{~mm}$ sterile wire (Dentaurum, Pforzheim, FRG) which did not cause additional endothelial damage and did not obstruct the arterial lumen. They were individually suspended in culture dishes (24 well, Greiner, The Netherlands) filled with $1 \mathrm{ml}$ Dulbecco's Eagle modified culture medium (Gibco, Paisly, UK) containing $2 \mathrm{mM}$ glutamine (Gibco), $100 \mu \mathrm{g} / \mathrm{ml}$ streptomycin (Pharmachemie, Haarlem, The Netherlands) and $100 \mathrm{IU} / \mathrm{ml}$ penicillin (Gist-Brocades, Delft, The Netherlands). The medium was supplemented with $20 \%$ fetal calf serum (Seralab, Sussex, UK) that had been dialyzed to strip it from low molecular weight $(<6 \mathrm{kD})$ contractile components $(\mathrm{dFCS})(\mathrm{De}$ Mey et al, 1989) and contained $1 \mu \mathrm{M}$ of the thymidine-analogue 5-bromo-2'deoxyuridine (BrdUrd; Sigma Chemicals, Saint Louis, Mo, USA). The arterial preparations were transferred daily to culture dishes filled with fresh medium. After 4 days of culture the arterial segments were rinsed for $60 \mathrm{~min}$ in drugfree Krebs Ringer bicarbonate solution, fixed in periodate-lysine-paraformaldehyde at $37^{\circ} \mathrm{C}$, embedded in paraffin and cross sectioned (4 $\mu \mathrm{m}$ thick). Following blockade of endogenous peroxidases, an indirect enzyme-labeled antibody technique with peroxidase conjugated second-step reagent was used to visualize nuclei that had incorporated BrdUrd during the culture period (Gratzner, 1982). The sections were counter stained with hematoxylin. To quantify the extent of DNA synthesis in the smooth muscle, the percentage of nuclear profiles that stained for peroxidase (BrdUrd L\%) was determined in the tunica media. No nuclear peroxidase staining was observed in sections of vessels that had been cultured in the absence of BrdUrd or in sections that had not been exposed to the primary antibody.

Fetal calf serum was used as a stimulus because in preliminary organ 
culture experiments single recombinant growth factors (PDGF-AA, PDGF-BB, IGF-1, IL-1 and TGF- $\beta$ ) were found to be far less effective. In previous experiments with arteries of adult WKY we observed that serum elicited a transient stimulation of nuclear incorporation of BrdUrd in the arterial smooth muscle. Observations were 5 to 8 times larger than those following culture in serum-free medium (De Mey et al, 1991 ${ }^{\mathrm{c}}$, 1989). The effect was already maximal in the presence of $2 \%$ serum and peaked during the third culture day (De Mey et al, 1991 a $1991^{\mathrm{c}}$; Boonen et al, 1991). In the present study we deliberately used $20 \%$ serum and 4 days of continuous exposure to BrdUrd to avoid that the interpretation of the findings would be complicated by differences in sensitivity and kinetics between the experimental groups. It should be taken into account, however, that continuous exposure to BrdUrd results in overestimation of the actual percentage of the cells that were stimulated to synthesize DNA since the label is distributed over daughter nuclei that result from mitosis (Boonen et al, 1991).

In additional experiments restricted to intact and denuded carotid arteries of 20-week old WKY and SHR, BrdUrd was replaced by ${ }^{3} \mathrm{H}$-thymidine $(0.2$ $\mu \mathrm{M}, 5 \mathrm{Ci} / \mathrm{mmol}$, Amersham, UK) and ${ }^{14} \mathrm{C}$-leucine $(18 \mu \mathrm{M}, 54 \mathrm{mCi} / \mathrm{mmol}$, Amersham, UK)(Berk et al, 1989). Separate arterial segments were exposed to the tracers during consecutive $24 \mathrm{~h}$ periods to evaluate changes with time during a 7 day period. Following exposure to the labeled precursors, the arterial preparations were rinsed during $30 \mathrm{~min}$ in oxygenated Krebs-Ringer bicarbonate solution at $37^{\circ} \mathrm{C}$. The amount of thymidine and leucine that was incorporated was determined by liquid scintillation following solubilization of the tissues in $1 \mathrm{~N} \mathrm{NaOH}$ and expressed relative to the DNA content measured by a fluorometric assay (Labarca and Paigen, 1980). In the case of leucine incorporation, the concentration of unlabeled amino-acid present in the culture medium $(0.63 \mathrm{mM})$ was taken into account. Because the concentration of endogenous thymidine was unknown, additional experiments were performed in the presence of $2.2 \mu \mathrm{M}$ thymidine, with the same level of radioactivity. Furthermore, for intact and denuded carotid artery segments of 20 week old WKY the incorporation of thymidine during the third culture day was evaluated in the presence of a range of exogenous thymidine concentration $(0-5.2 \mu \mathrm{M})$.

All data are shown as means \pm S.E.M. Statistical significance of differences was evaluated by analysis of variance followed by Scheffe's F-test for multiple comparisons (Wallenstein et al, 1980). 


\section{RESULTS}

\section{DNA synthesis determined by immunohistochemistry}

\section{Young and adult normotensive WKY rats}

Figure 5.1 illustrates BrdUrd L\% in the tunica media of isolated carotid and renal arteries of 6 and 20 week old WKY in the presence of endothelium and following its removal. At both ages, findings were significantly larger in renal than in carotid arteries. Effects of endothelium removal at 6 and 20 weeks of age were qualitatively similar in carotid and renal arteries (fig. 5.1). In arteries of young and adult WKY, endothelium removal increased BrdUrd L\% in the tunica media (fig. 5.1). This was also observed in arteries obtained from 20-week old WIS; BrdUrd L\% averaged $0.3 \pm 0.2$ and $8.0 \pm 2.7$ in the tunica media of carotid artery segments with and without endothelium, and $7.8 \pm 1.9$ versus $17.2 \pm 2.4$ in the tunica media of renal artery segments with or without endothelium, respectively. The immunocytochemical index of maximally stimulated DNA synthesis in the muscular layers of de-endothelialized WKY carotid and renal arteries was larger at 6 than at 20 weeks of age (fig. 5.1).

\section{Young and adult SHR}

BrdUrd $\mathrm{L} \%$ in the tunica media of carotid and renal arteries isolated from 6- and 20-week old SHR are shown in figure 5.2. Again, the observations were at both ages significantly larger in renal than carotid arteries. As observed for WKY, endothelium removal increased BrdUrd L\% in the carotid and renal artery media of 6 week old SHR (fig. 5.2). Yet, at 20 weeks of age, endothelium removal did not significantly alter BrdUrd L\% in the smooth muscle of SHR arteries (fig. 5.2).

At 6 weeks of age, intra-medial DNA synthesis was both in the absence and presence of endothelium comparable in renal arteries of WKY and SHR. In carotid arteries of 6 week old SHR, with or without endothelium, BrdUrd $\mathrm{L} \%$ was less marked but not statistically different from that in age-matched WKY. At 20 weeks of age, however, BrdUrd L\% in the media of SHR carotid and renal arteries was significantly smaller than that in the media of WKY arteries (figs. 5.1, 5.2).

\section{Hypertensive WKY rats}

Unlike in arteries of adult SHR, BrdUrd L\% in the tunica media of carotid and renal arteries isolated from the hypertensive body part of 20-week old aorta-coarcted WKY (AoH, fig. 5.3), did not differ from that in the corresponding arteries of 20-week old normotensive WKY (fig. 5.1). In 


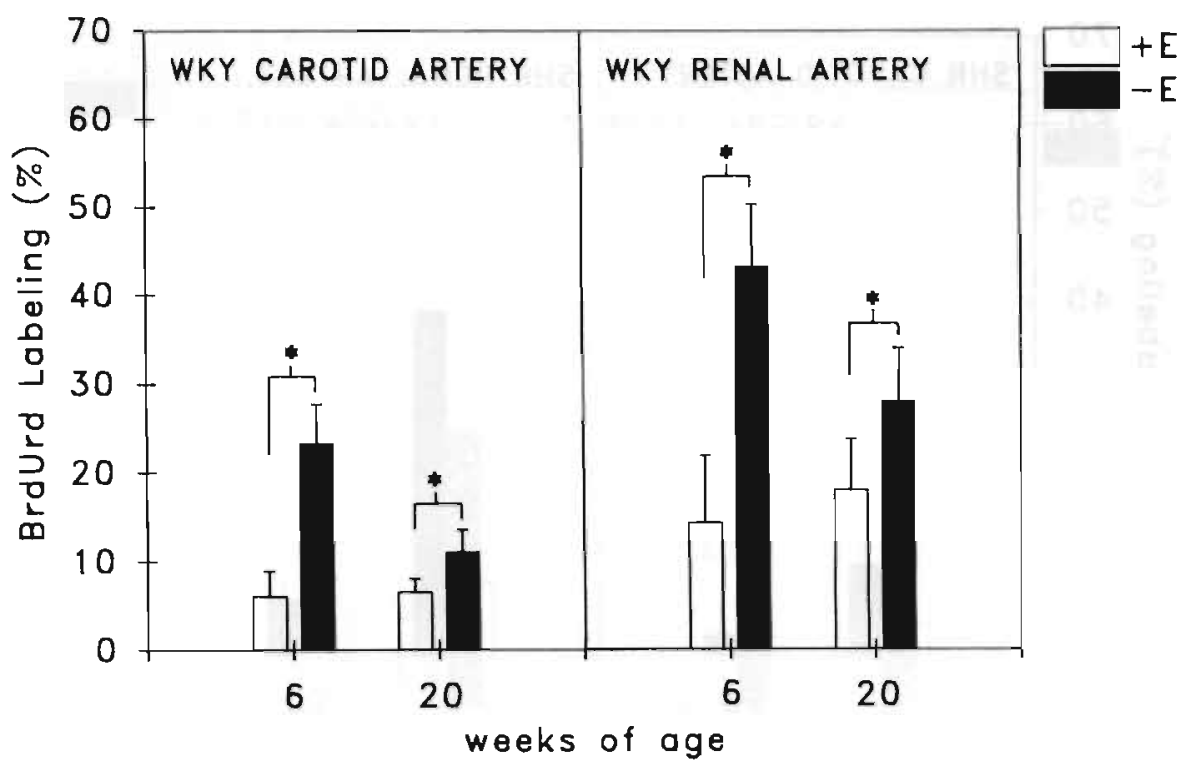

Figure 5.1 Effects of removal of endothelium on BrdUrd L\% in the tunica media of carotid and renal arteries isolated from 6 and 20 week old WKY rats $(n=8)$. Arterial segments were either left intact or mechanically denuded of endothelium, and maintained in culture for 4 days in the continuous presence of $20 \%$ dialyzed fetal calf serum (dFCS) and $1 \mu \mathrm{M}$ BrdUrd. Nuclear incorporation of BrdUrd is shown for arterial segments with ( $\square$ ) and without $(\square)$ endothelium. All differences between carotid and renal arteries are statistically significant at both ages $(p<0.05) .{ }^{*}$ Differences between preparations with and without endothelium are statistically significant $(p<0.05)$. All differences between denuded arteries of 6 and 20 week old WKY rats are statistically significant $(p<0.05)$.

addition, three weeks after aorta coarctation, removal of endothelium increased BrdUrd labeling of arterial smooth muscle cells in carotid and renal arteries that had been exposed to elevated blood pressure (fig. 5.3) as it did in control WKY arteries (fig. 5.1). This was even more marked in carotid arteries of aorta coarcted rats than in those of normotensive 20-week old WKY.

In renal arteries isolated from the lower body part of aorta coarcted WKY (Aoh), the effect of endothelium removal on DNA synthesis in arterial smooth muscle was not statistically significant (fig. 5.3). 


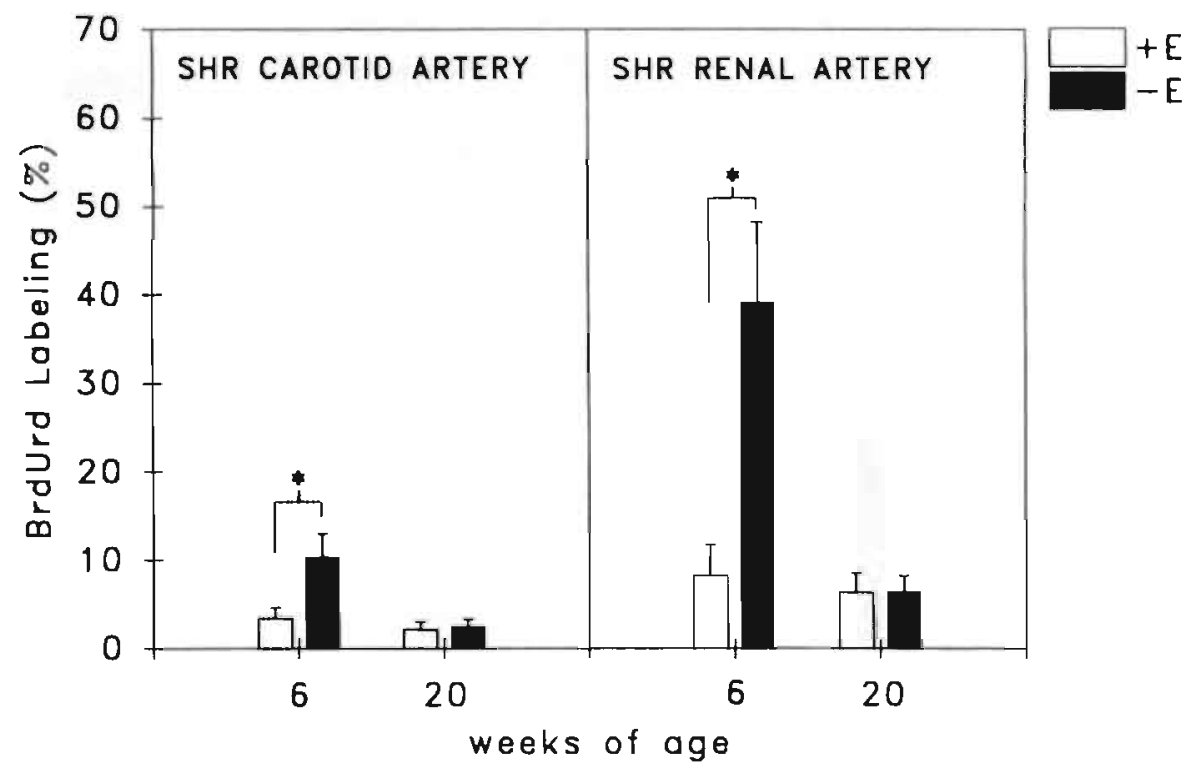

Figure 5.2 Effects of removal of endothelium on BrdUrd L\% in the tunica media of carotid and renal arteries isolated from 6 and 20 week old SHR $(n=8)$. Arterial segments were either left intact or mechanically denuded of endothelium, and maintained in culture for 4 days in the continuous presence of $20 \% \mathrm{dFCS}$ and $1 \mu \mathrm{M}$ BrdUrd. Nuclear incorporation of BrdUrd is shown for arterial segments with $(\square)$ and without ( $\square$ ) endothelium. All differences between carotid and renal arteries are statistically significant at both ages $(p<0.05) .{ }^{*}$ Difference between preparations with and without endothelium are statistically significant $(p<0.05)$. All differences between denuded arteries of 6 and 20 week old SHR are statistically significant $(\mathrm{p}<0.05)$.

\section{Hypertensive mouse Ren-2 transgenic rats}

Figure 5.4 illustrates BrdUrd L\% in the tunica media of isolated carotid and renal arteries of 11 week old SDR and TGR in the presence and absence of endothelium. In both strains, observations were again larger in renal than in carotid arteries. Endothelium removal increased BrdUrd L\% in the carotid artery of TGR and in the renal artery of both SDR and TGR (fig. 5.4). 


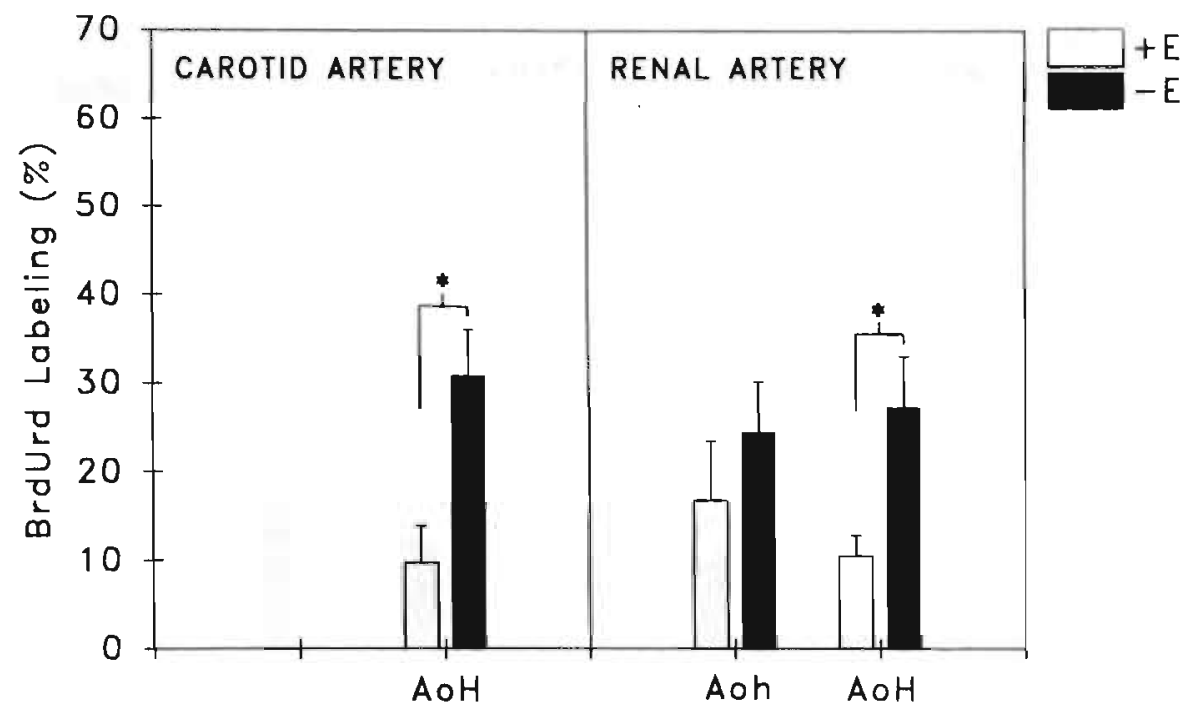

Figure 5.3 Effects of hypertension induced by aona coarctation on DNA synthesis in the tunica media of carotid and renal arteries of 20 week old WKY rats $(n=8)$. Arteries were isolated from the hyper-( $\mathrm{AOH}$ ) and hypotensive (Aoh) body part of aorta-coarcted WKY. BrdUrd labeling is shown for the tunica media of arterial segments after 4 days of culture with $(\square)$ and without ( $\square$ ) endothelium. * Difference between preparations with and without endothelium is statistically significant $(p<0.05)$.

\section{DNA and protein synthesis determined with labeled precursors}

Figure 5.5 illustrates the time course of incorporation of thymidine and leucine in intact and de-endothelialized carotid artery segments isolated from 20-week old WKY and SHR in the presence of 0.2 or $2.0 \mu \mathrm{M}$ thymidine. These findings confirm that in arteries of adult WKY, but not SHR, removal of endothelium significantly increases DNA synthesis. Incorporation of leucine, an index of overall protein synthesis, did, however, not differ between carotid artery segments with and without endothelium, or between preparations of both strains. Thymidine incorporation detected in the presence of $0.2 \mu \mathrm{M}$ of the nucleoside (fig. 5.5) was significantly larger in carotid artery segments of adult WKY than in those of adult SHR, as was suggested by the observations with BrdUrd (figs. 5.1, 5.2). In the presence of $2.2 \mu \mathrm{M}$ thymidine, however, 


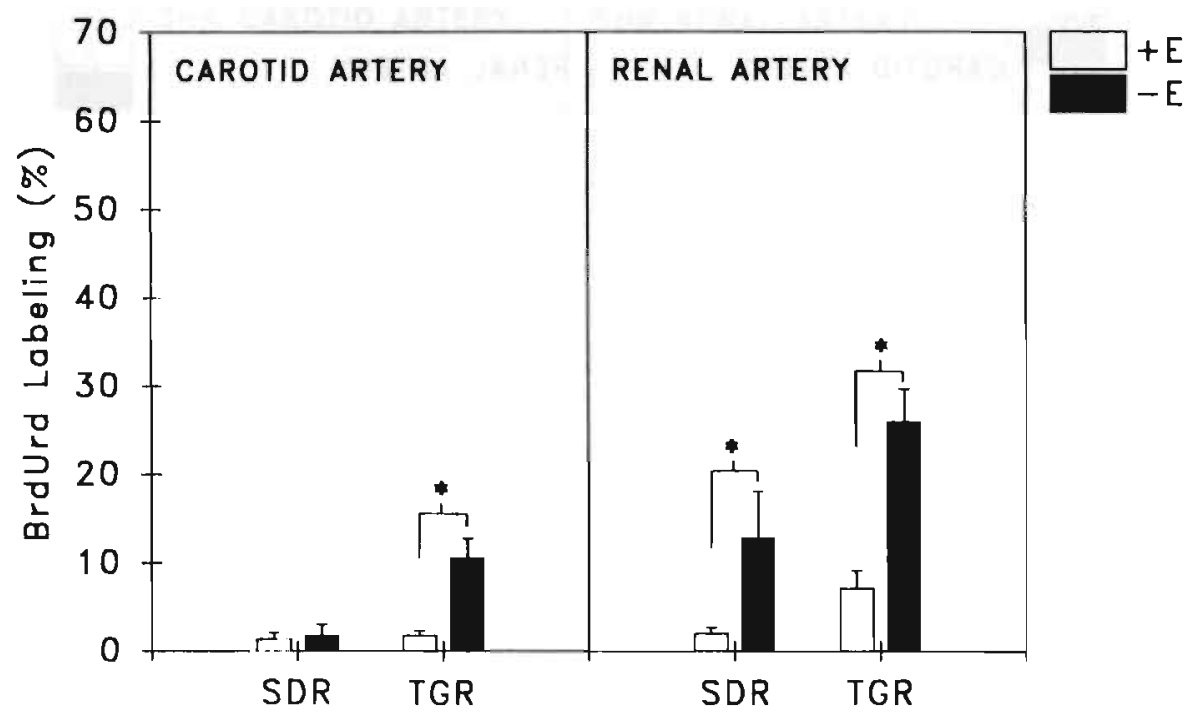

Figure 5.4 Effects of removal of endothelium on BrdUrd L\% in the tunica media of carotid and renal arteries isolated from 11 week old SDR $(n=4)$ and TGR $(n=8)$. Arterial segments were either left intact or mechanically denuded of endothelium, and maintained in culture for 4 days in the continuous presence of $20 \%$ dFCS and $1 \mu \mathrm{M}$ BrdUrd. Nuclear incorporation of BrdUrd is shown for arterial segments with $(\square)$ and without ( $\square$ ) endothelium. * Difference between preparations with and without endothelium are statistically significant $(p<0.05)$.

incorporation of the nucleoside did not differ between de-endothelialized carotid artery segments of adult WKY and SHR (fig. 5.5).

In WKY vessels, a stimulatory effect of endothelium removal on thymidine incorporation was evident in the presence of a broad concentration of exogenous thymidine (fig. 5.6). In carotid arteries of 20 week old SHR, however, endothelium removal did not alter thymidine incorporation in the presence of 0.2 or $2.2 \mu \mathrm{M}$ (fig. 5.5) of the nucleoside. 

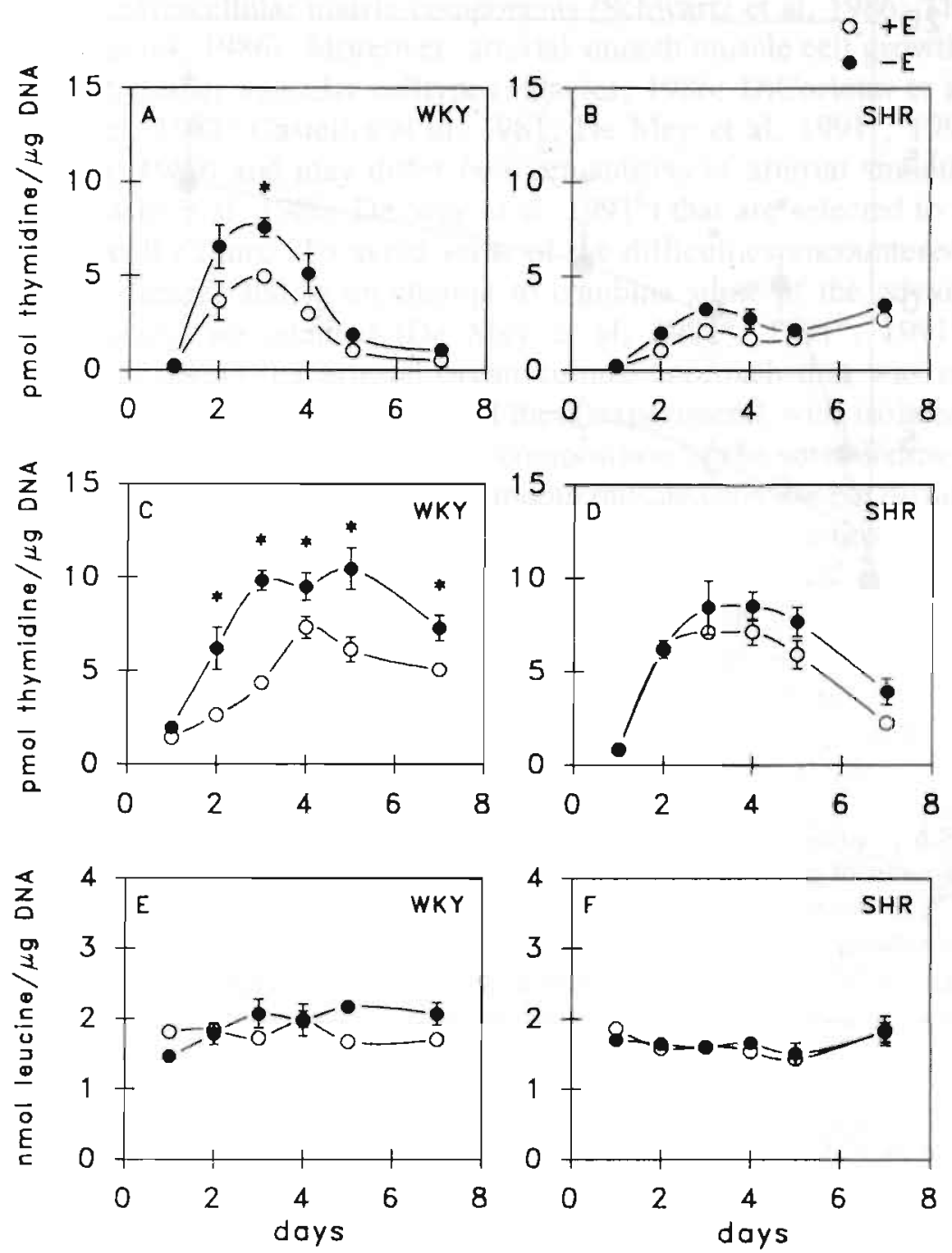

Figure 5.5 Effect of removal of endothelium on incorporation of ${ }^{3} \mathrm{H}$-thymidine (A,B,C,D) and ${ }^{14} \mathrm{C}$-leucin (E,F) in isolated carotid arteries of adult WKY (left) and SHR (right). Arterial segments were either left intact $(O)$ or denuded of endothelium (๑), and maintained in culture in the continuous presence of $20 \%$ dFCS. During a 7 day period separate arterial segments were exposed during consecutive $24 \mathrm{~h}$ periods to $0.2 \mu \mathrm{M}{ }^{3} \mathrm{H}$ thymidine ( $5 \mathrm{Ci} / \mathrm{mmol}$; top), or $2.2 \mu \mathrm{M}{ }^{3} \mathrm{H}$-thymidine $(0.45 \mathrm{Ci} / \mathrm{mmol}$; middle), and $18 \mu \mathrm{M}$ ${ }^{14} \mathrm{C}$-leucine $(54 \mathrm{mCi} / \mathrm{mmol}$; bottom). * Differences between preparations with and without endothelium are statistically significant $(p<0.05 ; n=6)$. 


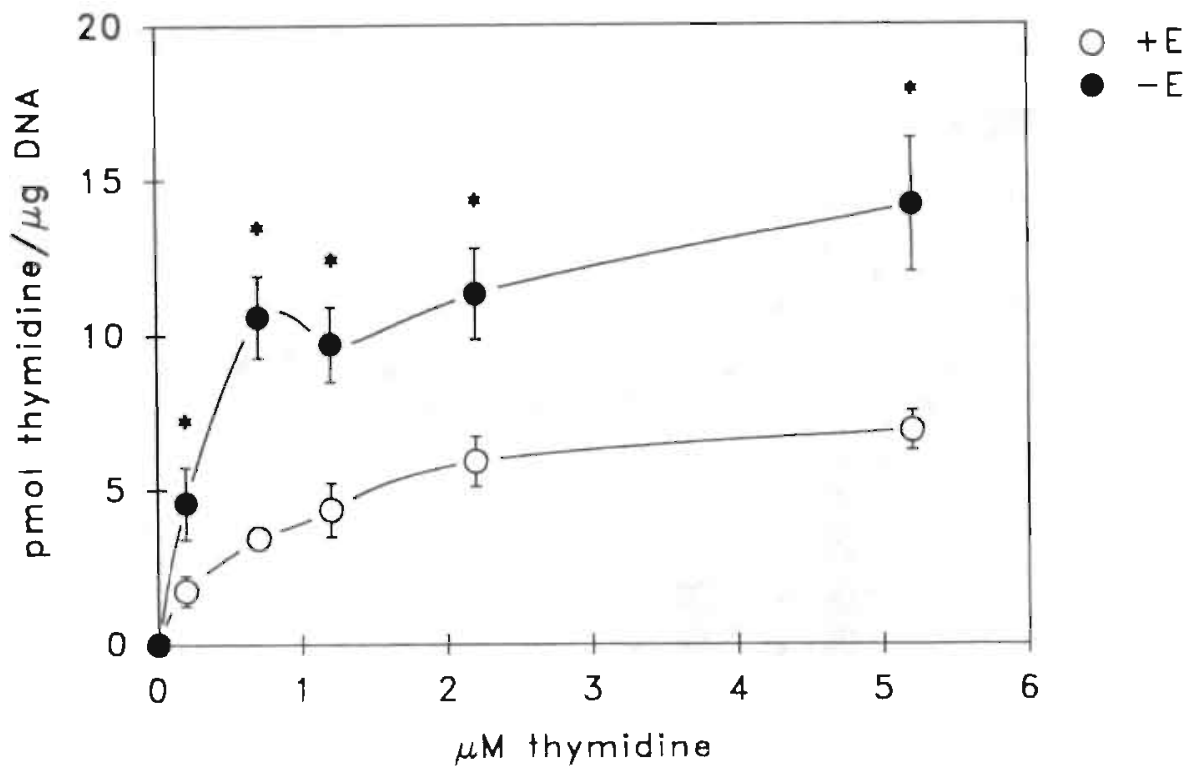

Figure 5.6 Effects of exogenous thymidine concentration on DNA synthesis in isolated carotid arteries of 20 week old WKY rats $(n=6)$ in vitro. Arterial segments were either left intact $(O)$ or mechanically denuded of endothelium $(\bullet)$, and maintained in culture in the continuous presence of $20 \% \mathrm{dFCS}$ during 3 days. Separate arterial segments were exposed during the last $24 \mathrm{~h}$ to $0.2-5.2 \mu \mathrm{M}$ exogenous thymidine. ${ }^{*}$ Differences between preparations with and without endothelium are statistically significant $(p<0.05)$.

\section{DISCUSSION}

The extent to which DNA synthesis could be stimulated in the smooth muscle of isolated arteries was affected by removal of the endothelium. In this respect differences were observed between young and adult rats and between vessels of normotensive and spontaneously hypertensive rats.

Increased DNA synthesis underlies hypertrophy of arterial smooth muscle in hypertension for as much as it is due to hyperploidy or hyperplasia of the arterial smooth muscle cells (Schwartz et al, 1986; Owens, 1989). The stimuli that are involved remain largely unknown. They have been addressed by experiments in vivo and in cell culture. In the former it is difficult to control the intensity and duration of mitogenic conditions and to dissociate local phenomena from changes in hemodynamics and in neurohumoral control. The 
latter are complicated by changes in cellular phenotype, cell density and presence of extracellular matrix components (Schwartz et al, 1986; Thyberg et al, 1990; Davies, 1986). Moreover, arterial smooth muscle cell growth may be influenced by other vascular celltypes (Davies, 1986; DiCorletto et al, 1983; Warner et al, 1987; Castellot et al, 1981; De Mey et al, 1991 ${ }^{\mathrm{b}}, 1991^{\mathrm{c}}$; Koo and Gotlieb, 1989) and may differ between subsets of arterial smooth muscle cells (Schwartz et al, 1986; De Mey et al, $1991^{\mathrm{c}}$ ) that are selected to different extends by cell culture. To avoid some of the difficulties encountered in vivo and in cell culture, and in an attempt to combine some of the advantages of both techniques, we adapted (De Mey et al, 1991 a $1991^{\mathrm{b}}, 1991^{\mathrm{c}}, 1989$; Boonen et al, 1991) the arterial organ culture approach that was originally introduced by Mauger et al (1975). In these experiments with isolated arterial segments, the physical conditions and composition of the surrounding medium are maintained constant. The arterial smooth muscle cells are not dispersed and thus remain at high density and in their normal microenvironment. DNA synthesis can be stimulated without prior loss of contractility (De Mey et al, $1991^{\mathrm{a}}, 1991^{\mathrm{c}}, 1989$; Boonen, 1991) and, as observed in the present study, without profound changes in protein synthesis, ruling out major changes in cellular phenotype (Schwartz et al, 1986; Thyberg et al, 1990).

Radiochemical and immunocytochemical methods were used to quantify incorporation of thymidine and of a thymidine-analogue (BrdUrd), respectively. Cytochemistry allows to separately document responses of medial smooth muscle cells. The radiochemical method, on the other hand, yields an observation for the entire arterial wall. This may account for the differences between results of both approaches. Conversion of our observations to the actual extent of DNA synthesis requires knowledge of the kinetics of karyokinesis and of the size of the endogenous thymidine pool. This information is not available for blood vessels. Values are thus to be considered as indices rather than as true measures of DNA synthesis.

The levels of BrdUrd labeling which we observed in both types of isolated artery were larger than those found in intact animals. Differences range between 1-4 fold ( 6 week old SHR and WKY; 40) and more than 200 fold (20 week old SHR and WKY; Schwartz et al, 1986; Clowes et al, 1989) suggesting marked stimulation in vitro. In the present study we deliberately used this strong multifactorial stimulation procedure to exclude differences in sensitivity for specific growth factors and growth inhibitors between experimental groups (Hadrava et al, 1989; Resink et al, 1987, 1989) and to concentrate on differences in near maximal response.

Part of the experiments were performed in arteries that had been both sympathectomized and denuded of endothelium, to study arterial smooth muscle in the absence of neurogenic (Head, 1991; Donohue et al, 1989) and endothelial (Davies, 1986; De Mey et al, 1991 a $1991^{\mathrm{C}}$ ) modulatory influences. 
As noted earlier (De Mey et al, $1991^{\mathrm{c}}$ ), DNA synthesis could be stimulated to a larger extent in renal than carotid artery smooth muscle. This was the case for young and adult WKY, WIS, SDR, SHR and TGR. The regional heterogeneity of this response contrasts with the observation that smooth muscle cells isolated from renal and carotid arteries of adult WKY (De Mey et al, $1991^{c}$ ) and SHR (unpublished observations) have similar population doubling times during cell culture. We have proposed that this could be due to regional heterogeneity with respect to the presence of (i) growth inhibitory influences in the arterial tunica media and (ii) subsets of growth-prone and growth-resistant smooth muscle cells (Schwartz et al, 1986; De Mey et al, $1991^{\mathrm{c}}, 1991^{\mathrm{d}}$; Boonen et al, 1991).

While BrdUrd labeling of the arterial tunica media was comparable in sympathectomized and denuded arteries of young WKY and SHR, it was markedly lower in those of adult SHR compared to those of adult WKY or WIS. BrdUrd labeling in arteries of WKY rendered hypertensive by aorta coarctation and in arteries of TGR were, however, if anything more marked than in those of normotensive rats. These findings could suggest a selective and pressure independent alteration of the growth characteristics of arterial smooth muscle in adult SHR. Our observations are opposite to the repeatedly described hyperkinetic proliferation of smooth muscle cells isolated from the thoracic aorta of SHR (Hadrava et al, 1989; Resink et al, 1987; Schwartz et al, 1986; Owens, 1989). Reduced mitogenicity of arterial smooth muscle of adult SHR may be the consequence of elevated intra-arterial DNA synthesis in situ during earlier developmental stages (De Mey et al, $1991^{\mathrm{d}}$ ). Observations with ${ }^{3} \mathrm{H}-$ thymidine tend to suggest, however, that differences in nucleoside metabolism may be responsible for the quantitative difference in BrdUrd labeling of the arterial tunica media of adult SHR and normotensive rats. While the interstrain difference could be confirmed in the presence of $0.2 \mu \mathrm{M}$ thymidine, a concentration which most authors use for in vitro experimentation (Hadrava et al, 1989; Resink et al, 1987, 1989; Berk et al, 1989), thymidine incorporation did not differ between denuded carotid arteries of adult SHR and WKY exposed to $2.2 \mu \mathrm{M}$ thymidine. Thus, a major difference in availability of endogenous thymidine may be responsible for differences in apparent DNA synthesis between denervated de-endothelialized arteries of SHR and normotensive rats.

Observations in arteries of young SHR, did not differ from those in vessels of young WKY. In both carotid and renal arteries of adult SHR, on the other hand, endothelium removal did not significantly affect BrdUrd labeling or thymidine incorporation. This was the case in the presence of both a low and a high concentration of exogenous thymidine. Whether resistance to endothelium removal is due to different properties of the endothelium or different growth control of arterial smooth muscle in arteries of adult SHR, 
remains to be established. The observation that in arteries of adult SHR endothelium-removal failed to affect DNA synthesis is, in general, in line with reduced endothelium-dependent relaxing responses in arteries of hypertensive rats (Vanhoutte, 1989; De Mey and Gray, 1985; Lüscher, 1990). However, alterations of endothelial modulation of arterial tone, (i) have been documented in experimental models of both essential and secondary hypertension, (ii) reach statistical significance only after prolonged elevation of blood pressure, and (iii) can be reversed by various antihypertensive interventions (Vanhoutte, 1989;De Mey and Gray, 1985; Lüscher, 1990). They, thus, most likely represent a secondary adaptation to the hypertension. This may not be the case for endothelial inhibition of intra-arterial DNA synthesis. While endothelium removal did not affect DNA synthesis in arteries isolated from adult SHR, it increased BrdUrd labeling in arteries of WKY that had been made hypertensive by aorta-coarctation and in arteries of TGR. Thus, the lack of effect of endothelium removal on medial DNA synthesis in adult SHR is not likely to represent an adaptation to elevated blond pressure.

The mechanism by which the endothelium may interfere with stimulated growth of arterial smooth muscle is largely unknown. Endothelium removal promoted DNA synthesis but did not affect incorporation of ${ }^{3} \mathrm{H}$-leucine, an index of overall protein synthesis. This suggest that the endothelium may selectively inhibit cellular proliferation while not affecting increases in cell size. Endothelial influences may thus promote arterial smooth muscle cell hypertrophy when the arterial wall is exposed to mitogenic conditions. The nature of the endothelial influence involved herein is unknown. Potential endothelium-derived inhibitors include heparin (Casteliot et al, 1981), interleukin 1 and TGF- $\beta$ (Schiffers et al, submitted). These are more likely to be involved in the present findings than either NO or prostaglandins (De Mey et al, $1991^{\mathrm{a}}$; Schiffers et al, accepted). In previous experiments, DNA synthesis in rat arteries with endothelium was not increased by L-NAME, arginine removal, methylene blue or indomethacin (De Mey et al, 1991 ${ }^{\mathrm{a}}$; Schiffers et al, submitted). Future analysis of candidate mediators may be helped by taking into account selective inhibition of DNA synthesis and the differential behavior of arteries of adult SHR.

In summary, stimulated DNA synthesis was measured in isolated arteries of several rat strains. Findings were for all strains more marked in renal than carotid arteries. Estimated DNA synthesis was considerably smaller in arteries of adult SHR, than in those of young SHR, various normotensive strains and other models of hypertension. This may result from altered thymidine metabolism. In arteries of all strains and models, except adult SHR, DNA synthesis was more marked in the absence of endothelium than in its presence. These findings confirm inhibitory influences of endothelium on arterial smooth muscle cell proliferation in the rat and suggest that this mechanism may be 
CHAPTER 5

altered in SHR independently of blood pressure. 


\section{Chapter}

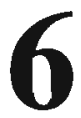

Effects of Candidate Autocrine and Paracrine Mediators on Growth Responses in Isolated Arteries of the Rat

P.M.H. Schiffers, G.E. Fazzi, D. van Ingen Schenau and J.G.R. De Mey

Department of Pharmacology, University of Limburg, Maastricht, the Netherlands. (Arteriosclerosis and Thrombosis, accepted) 


\section{INTRODUCTION}

Mechanisms of arterial smooth muscle cell (ASMC) growth control have been studied primarily by cell culture and by balloon-catheter-injury in experimental animals (Schwartz et al, 1986; Chamley-Campbell et al, 1979; Clowes et al $1983^{\mathrm{b}}$, Jackson and Schwartz, 1992). These approaches indicated that vascular cells can produce peptidergic growth factors, cytokines and vasoactive agents that can interfere with growth responses (Warner et al, 1987a; Sjölund et al, 1988; Clemmons et al, 1983; Gospodarowicz et al, 1988; Hahn et al, 1990). This is not restricted to ASMC but applies to endothelial cells as well (Warner et al, 1987b; Hannan et al, 1988; Vlodavsky et al, 1987; Bubin et al, 1989; Gryglewski et al, 1988; Moncada and Higgs, 1991). A role for autocrine and paracrine mediators in vascular growth responses depends not only on their production but also on the responsiveness of the cell types involved (Bondjers et al, 1991). Many agents have been shown to promote proliferation of isolated ASMC in vitro (Davies, 1986; Libby et al, 1988; Ross et al, 1986; Klagsbrun and Edelman, 1989; Bubin et al, 1989; Geisterfer et al, 1988 ). Yet, this does not necessarily predict their activity in the vascular wall (Jawien et al, 1992; Olson et al, 1992). Several causes have been suggested to underlie different effects of growth factors in isolated and intravascular ASMC. These include (i) differences in cell density, phenotype and presence of extracellular matrix components (Thyberg et al, 1991) and (ii) the multifactorial nature of ASMC growth control in situ including not only growth factors but also growth inhibitors (Thyberg et al, 1991, Owens et al, 1988 ${ }^{\mathrm{b}}$; Morasaki et al, 1988; Southgate and Newby, 1990). In generai, analysis of the effects of exogenous agents and contributions of endogenous factors to growth responses in vivo are complicated by the lack of sufficient agonists, the inavailability of selective inhibitors and the occurrence of both systemic and local responses during drug treatment. 'Furthermore, influences of the endothelium on the underlying ASMC (De Mey et al 1991 a $1991^{\mathrm{c}}$; Koo and Gotlieb, 1989) may interfere with structural responses of the vascular wall to administered agents.

In this study we, therefore, evaluated effects of candidate autocrine and paracrine mediators on DNA synthesis and cellular proliferation in isolated arteries during organ culture (De Mey et al, 1989; Boonen et al, 1991). In this experimental system (i) ASMC are not dispersed and thus remain at high density and in contact with their natural extracellular matrix, (ii) both cell kinetics, and changes of arterial structure can be studied, (iii) known concentrations and combinations of growth factors can be applied and (iv) the experiments can be performed in the absence and presence of endothelium. The choice of the agents that were used, which included peptidergic growth factors, cytokines and vasoactive agents, was inspired by their action on ASMC proliferation during cell culture (Davies, 1986; Libby et al, 1988; Ross et al, 
1986; Klagsbrun and Edelman, 1989; Bubin et al, 1989; Geisterfer et al, 1988) and by the possibility that they can be delivered to the arterial wall by the ASMC themselves (Warner et al, 1987a; Sjölund et al, 1988; Clemmons et al, 1983; Gospodarowicz et al, 1988; Hahn et al, 1990) and by endothelial cells (Warner et al, 1987b; Hannan et al, 1988; Vlodavsky et al, 1987; Bubin et al, 1989; Gryglewski et al, 1988; Moncada and Higgs, 1991).

\section{MATERIALS AND METHODS}

The experiments were performed in segments of carotid and renal arteries that had been isolated from 20 week old male Wistar-Kyoto (WKY) rats and chemically sympathectomized in vitro with 6-hydroxydopamine (300 $\mu \mathrm{g} / \mathrm{mi}$; Sigma Chemicals St.Louis, MO, USA)(Aprigliano and Hermsmeyer, 1976; De Mey et al, 1989). Except when specifically mentioned, the segments were mechanically denuded of endothelium by passing the shaft of a hypodermic needle (outer diameter 0.8 and $0.7 \mathrm{~mm}$ for carotid and renal arteries, respectively) through the arterial lumen. Removal of the endothelium was confirmed by the absence of luminal immunoreactivity for von Willebrand factor and angiotensin-converting-enzyme and by the absence of relaxing responses to acetylcholine and histamine. We previously demonstrated that denudation did not appreciably affect the DNA content of the arterial preparations or their maximal contractile responses to high potassium (De Mey et al, $1991^{\mathrm{a}}$ ), indicating that no medial injury was induced.

\section{Tissue culture}

Arteriai organoid culture was performed as previously described (De Mey et al, 1989, 1991 a $1991^{\circ}$, Boonen et al, 1991). All segments were mounted on a $0.25 \mathrm{~mm}$ sterile wire (Dentaurum, Pfortzheim, GER) and individually suspended in culture dishes (24 well, Greiner, The Netherlands) filled with $1 \mathrm{ml}$ Dulbecco's minimal Eagle's medium (L-arginine content: 250 $\mu \mathrm{M}$; Gibco, Paisly, UK), containing $2 \mathrm{mM}$ glutamine (Gibco, Paisly, UK), 100 $\mathrm{IU} / \mathrm{ml}$ penicillin (Gist-Brocades, Delft, The Netherlands), $100 \mu \mathrm{g} / \mathrm{ml}$ streptomycin (Pharmachemie, Haarlem, The Netherlands), and either $20 \%$ dialyzed fetal calf serum (dFCS, Seralab, Sussex, UK)(De Mey et al, 1989), 5\% rat plasma derived serum (PDS), or $5 \%$ plasma derived serum stripped from progression factors (CMS-PDS)(Vogel et al, 1978). Arterial preparations were maintained under these conditions at $37^{\circ} \mathrm{C}$ in an atmosphere of $95 \%$ air and $5 \%$ $\mathrm{CO}_{2}$ at $97 \%$ humidity. 


\section{Short-term tissue culture}

To evaluate the effects of exogenous growth factors on medial DNA synthesis, renal arteries were maintained in culture for 3 days in the presence of $20 \% \mathrm{dFCS}$, 5\%PDS, or 5\% CMS-PDS, supplemented with either $1 \mu \mathrm{M}$ Angiotensin II (Ang II), $10 \mathrm{nM}$ endothelin-1 (ET-1), $5 \mathrm{ng} / \mathrm{ml}$ platelet-derived growth factor (PDGF AA or PDGF BB), $100 \mathrm{nM}$ insulin-like growth factor 1 (IGF-1), $100 \mathrm{U} / \mathrm{ml}$ interleukin-1 (IL-1), $10 \mathrm{ng} / \mathrm{ml}$ transforming growth factor beta (TGF- $B$ ), or $10 \mathrm{ng} / \mathrm{ml}$ basic fibroblast growth factor (bFGF). The concentrations of these mediators have been reported to be maximal with respect to stimulation of ASMC proliferation during cell culture (Schwartz et al, 1986; Libby et al, 1988; Ross et al, 1986; Klagsbrun and Edelman, 1989; Owens et al, 1988 ${ }^{\mathrm{b}}$; Bubin et al, 1989; Geisterfer et al, 1988). Combinations of IGF-1 with the other agents were also studied. In additional experiments, concentration response curves were constructed for TGF- $\beta(0.5-10 \mathrm{ng} / \mathrm{ml})$ and $\mathrm{bFGF}(0.1-10 \mathrm{ng} / \mathrm{ml})$. All experiments were performed in the continuous presence of $1 \mu \mathrm{g} / \mathrm{ml}$ indomethacin, a cyclooxygenase inhibitor.

To estimate DNA synthesis, $1 \mu \mathrm{M}$ of the thymidine analogue 5-bromo2'-deoxyuridine (BrdUrd, Sigma Chemicals) was included in the culture medium with daily replacement of the medium and label. After 3 days of culture, the arterial segments were rinsed for $60 \mathrm{~min}$ in drug-free Krebs Ringer bicarbonate solution, fixed in periodate-lysine-paraformaldehyde at $37^{\circ} \mathrm{C}$, embedded in paraftin and cross sectioned ( $4 \mu \mathrm{m}$ thick). Nuclear incorporation of BrdUrd was evaluated by immunohistochemistry on cross-sections (Gratzner, 1982; De Mey et al, 1989). To quantify the extent of DNA synthesis, the percentage of nuclear profiles that stained for BrdUrd was determined in the tunica media.

In the present study, we confirmed that medial DNA synthesis was significantly larger in arterial segments denuded of endothelium than in intact arteries (De Mey et al, 1991 a $1991^{\mathrm{c}}$ ). To evaluate the involvement of nitric oxide (NO), DNA synthesis was measured in carotid arteries that were either left intact or denuded of endothelium and that had been exposed to $20 \%$ dFCS during 3 days of culture in the absence or presence of L-arginine $(250 \mu \mathrm{M})$ or $\mathrm{N}^{\mathrm{G}}$-nitro-L-arginine methyl ester (L-NAME; $200 \mu \mathrm{M}$ ). In these experiments, also the production of nitrite (a stable degradation product of NO) was measured in the conditioned media (Stuehr and Nathan, 1989). In brief, 500 $\mu \mathrm{l}$ samples were mixed with an equal volume of Griess reagent $(0.5 \%$ sulfanilamide/0.05\% $\mathrm{N}$-(1-naphthyl)ethylene-diamine dihydrochloride) and incubated at room temperature for 10 minutes. The optical densities were measured at $550 \mathrm{~nm}$ and $\mathrm{NO}_{2}^{-}$- concentrations were determined using $\mathrm{NaNO}_{2}$ as a standard (1-100 $\mu \mathrm{M})$ and water as a blank. Background $\mathrm{NO}_{2}^{-}$values of media were determined and subtracted from the experimental values. 


\section{Long term tissue culture}

We have previously shown that incubation of denuded arterial segments for up to 14 days in $20 \%$ dFCS resulted in the development of new layers of cells, displaying smooth muscle-like properties (De Mey et al, 1991 ${ }^{\mathrm{c}}$ ). To evaluate whether exogenous growth factors could influence this cellular proliferation, denuded renal arteries were exposed to $20 \%$ dFCS for 4 days, directly followed by 14 days of culture in 5\% CMS-PDS in the presence of 1 $\mu \mathrm{g} / \mathrm{ml}$ indomethacin and potential growth factors. The arterial preparations were transferred to culture dishes filled with fresh medium after 1-4, 6 and 13 days. To estimate cellular proliferation, the number of cells appearing at the adventitial edge of the vessel wall were counted after 14 days of culture.

\section{Sera and agents}

Fetal calf serum, a classic but undefined source of growth factors, appeared to contain high concentrations of serotonin (De Mey et al, 1989) and angiotensins (unpublished observations). For this reason, the serum was dialyzed (cut off, $6 \mathrm{kDa})$ against 3 changes of phosphate buffer $(\mathrm{pH}=7.4)$ at $4^{\circ} \mathrm{C}$ during $24 \mathrm{hr}$.

Both, PDS and CMS-PDS, were obtained by a method described by Vogel et al (1978). In brief, rat anticoagulated blood (sodium citrate) was spun at $3000 \mathrm{rpm}$ for $20 \mathrm{~min}$ at $4^{0} \mathrm{C}$. The supernatant was respun at $13500 \mathrm{rpm}$ for $20 \mathrm{~min}$ at $4^{0} \mathrm{C}$ to remove the platelets. After the second spin, $1.0 \mathrm{M} \mathrm{CaCl}_{2}$ was added to a final concentration of $20 \mathrm{mM}$, and Krebs Ringer bicarbonate solution (composition in $\mathrm{mM}$ : $\mathrm{NaCl} 118.5 ; \mathrm{KCl} 4.7 ; \mathrm{MgSO}_{4} 1.2 ; \mathrm{KH}_{2} \mathrm{PO}_{4} 1.2$; $\mathrm{NaHCO}_{3} 25.0 ; \mathrm{CaCl}_{2}$; glucose 11.1 ) was added in a ratio of $1 \mathrm{vol}$ to $6 \mathrm{vol}$ of plasma. The plasma was then incubated at $37^{\circ} \mathrm{C}$ for $2 \mathrm{hr}$ to allow clot formation and spun at $13500 \mathrm{rpm}$ for $30 \mathrm{~min}$ at $4^{\circ} \mathrm{C}$. The supernatant was dialyzed against three changes of $0.1 \mathrm{M}$ Tris-HCL $(\mathrm{pH}=7.4)$ at $4^{0} \mathrm{C}$ during 24 hr. Part of this plasma derived serum (PDS) was then applied to a CM Sephadex column $(50 * 2 \mathrm{~cm})$ and approximately 8 times the volume of fluid that was applied to the column was collected using $0.01 \mathrm{M}$ Tris- $\mathrm{HCl} / 0.09 \mathrm{M} \mathrm{NaCl}$ $(\mathrm{pH}=7.4)$ as eluents. The eluate was concentrated back to the original volume. This CMS-PDS was dialyzed against three changes of KRB during $24 \mathrm{hr}$. Both, CMS-PDS and PDS, were heated to $56^{\circ} \mathrm{C}$ for $30 \mathrm{~min}$, centrifuged at $3000 \mathrm{rpm}$ at $4^{\circ} \mathrm{C}$ for $20 \mathrm{~min}$, filtered and stored at $-70^{\circ} \mathrm{C}$ until use.

The homodimers of PDGF, and endothelin-1 were obtained from Bachem (Bubendorf, Switzerland). TGF-B and bFGF were purchased from Peninsula Laboratories (St. Helens, UK). Ang II and L-NAME were obtained from Sigma Chemicals (St.Louis, MO, USA). L-arginine free medium was prepared by Gibco (Paisly, UK). IGF-1 was a kind gift of Dr. K. Hofbauer (Ciba Geigy, 
Basle, Switzerland). IL-1 was kindly supplied by Dr. W Buurman (University of Limburg, Maastricht, the Netherlands).

\section{Statistics}

Data are shown as means \pm SEM. Statistical significance of differences was evaluated by one or two way analysis of variance where applicable. For multiple preplanned comparison, the ANOVA was followed by Bonferroni's $t$ test (Wallenstein et al, 1980). $\mathrm{p}<0.05$ was taken to denote statistical significance of differences.

\section{RESULTS}

\section{Basal and Maximal Responses in Denuded Vessels}

\section{DNA synthesis during short term culture}

Figure 6.1 illustrates the extent of nuclear incorporation of BrdUrd in the media of denuded renal artery segments exposed for 3 days to different media. Significant BrdUrd labeling was already obtained in serum-free medium. The presence of sera further stimulated intra-arterial DNA synthesis. The effect was larger for $20 \% \mathrm{dFCS}$ ( 10 to 15 fold) than for $5 \%$ rat PDS (5 to 8 fold). Part of the stimulatory action of PDS could be removed by passage over a Sephadex column (CMS-PDS, 2 to 4 fold stimulation above that seen in serum-free medium). Also in denuded carotid artery segments, BrdUrd labeling of the media was (i) significant after 3 days of incubation in serum-free medium

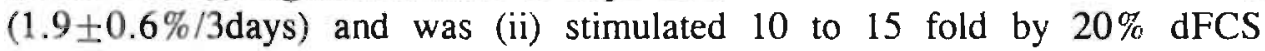
$(12.2 \pm 2.5 \% / 3$ days). Note that both findings were significantly lower in carotid than in renal arteries $(5.2 \pm 1.1 \% / 3$ days and $38.0 \pm 3.6 \% / 3$ days in the absence and presence of $\mathrm{dFCS}$, respectively).

\section{Cell proliferation during long term culture}

As previously reported (De Mey et al, $1991^{c}$ ), culture in the presence of $20 \%$ dFCS for up to 2 weeks did not alter the number of cells in the original media of renal or carotid artery segments, but gave rise to the formation of new layers of cells outside the original arterial wall. Figure 6.2 shows that the formation of this periadventitial layer was serum dependent. After 4 days of culture in the presence of $20 \%$ dFCS, a small number of cells could already be detected in cross sections of carotid arteries. When at this stage $20 \% \mathrm{dFCS}$ was removed or replaced by $5 \%$ CMS-PDS the growth of the new layer was prevented. In the continuous presence of $20 \%$ dFCS, however, the size of the 


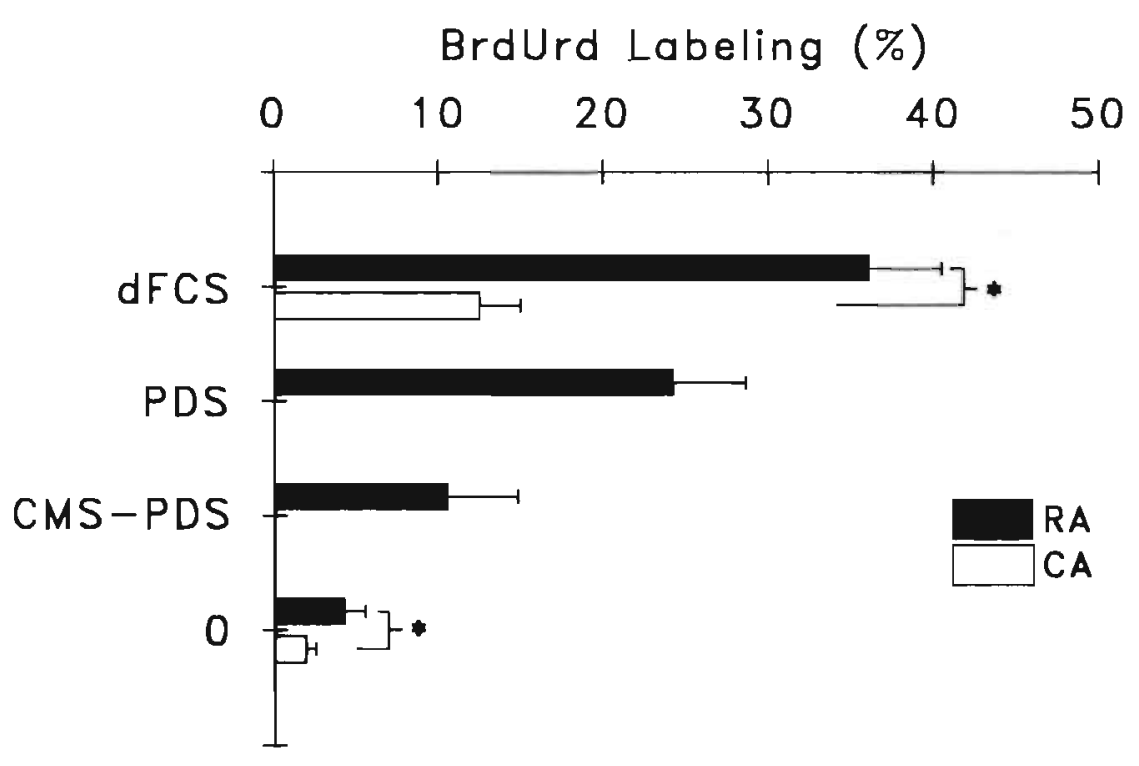

Figure 6.1 Effects of different sera on medial DNA synthesis in isolated and denuded renal ( $\square$ ) and carotid ( $\square$ ) arteries during 3 days of organ culture. The percentage of BrdUrd labeling is shown for $20 \% \mathrm{dFCS}, 5 \% \mathrm{PDS}, 5 \% \mathrm{CMS}$-PDS and serum-free medium. Data are expressed as means \pm SEM $(n=6)$. * Differences between renal and carotid arteries are statistically significant $(\mathrm{p}<0.05)$.

new layer increased markedly. After two weeks of incubation the number of cells in the new layer $(987 \pm 59$ /cross section) was markedly larger than that in the original media $(321 \pm 34$ /cross section).

\section{Exogenous Growth Factors and Medial DNA synthesis in Denuded Vessels}

Effects of high concentrations of recombinant growth factors on BrdUrd labeling were evaluated during mild (CMS-PDS), moderate (PDS) and strong 

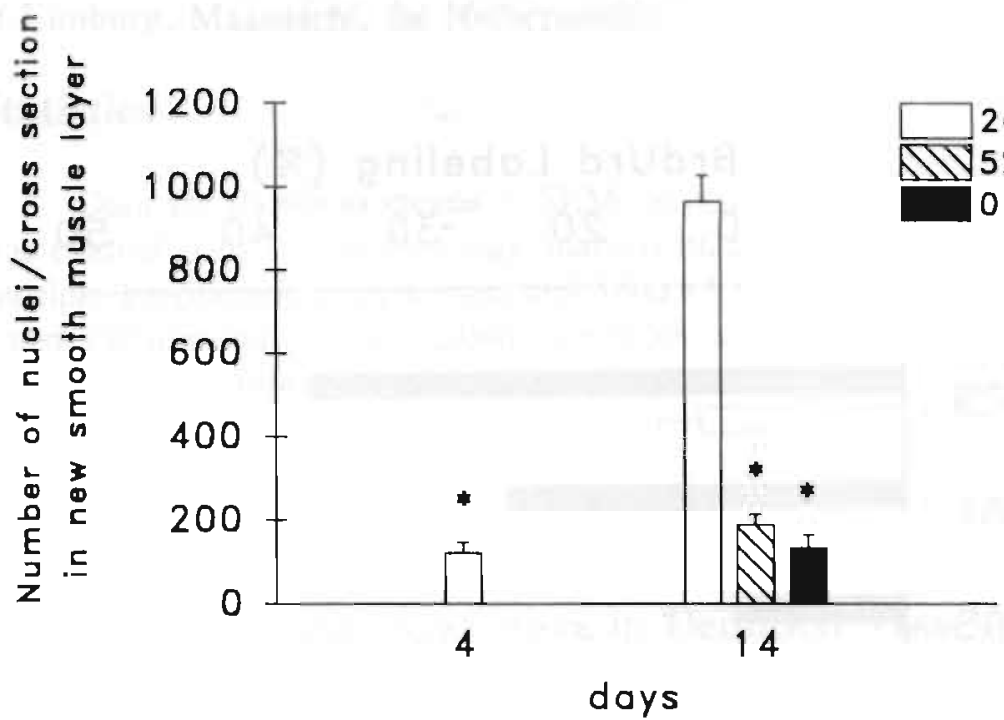

Figure 6.2 Effects of short and long-term term arterial tissue culture on the number of ASMC nuclei in the newly formed ASMC layer at the adventitial edge of the vessel wall. Denuded carotid arteries were either exposed to $20 \%$ dFCS during 4 or 14 days of culture ( $\square$ ), or to $20 \%$ dFCS during the first 4 days and subsequently to serum-free medium (ם) or $5 \%$ CMS-PDS (\$). Data are expressed as number of nuclei/cross section in the newly formed ASMC layer (means \pm SEM). * Differences from observations during continuous exposure to dFCS for 14 days of culture are statistically signiticant $(p<0.05)$.

(dFCS) stimulation. Furthermore, the agents were also tested in combination with IGF-1. These findings are summarized in figure 6.3.

In denuded rat renal artery exposed to CMS-PDS, TGF- $B$ and IL-1 reduced BrdUrd labeling. ET-1 and bFGF significantly stimulated DNA synthesis under these conditions, but PDGF AA, PDGF BB and Ang II were without effect (fig. 6.3)

By itself IGF-1 moderately and insignificantly enhanced DNA synthesis in CMS-PDS. It did not affect the inhibitory action of TGF- $B$ but abolished the inhibitory action of IL-1 on BrdUrd labeling of medial cells. Furthermore, IGF-1 unmasked a significant stimulatory effect of PDGF AA, but not PDGF BB or Ang II. In the presence of IGF-1, ET-1 and bFGF further increased DNA synthesis (fig. 6.3).

The relatively strong stimulation of DNA synthesis noted in the presence of PDS was reduced by TGF- $B$ and IL-1, and increased by bFGF. It was not affected by PDGF AA, PDGF BB, Ang II or ET-1 (fig. 6.3). 


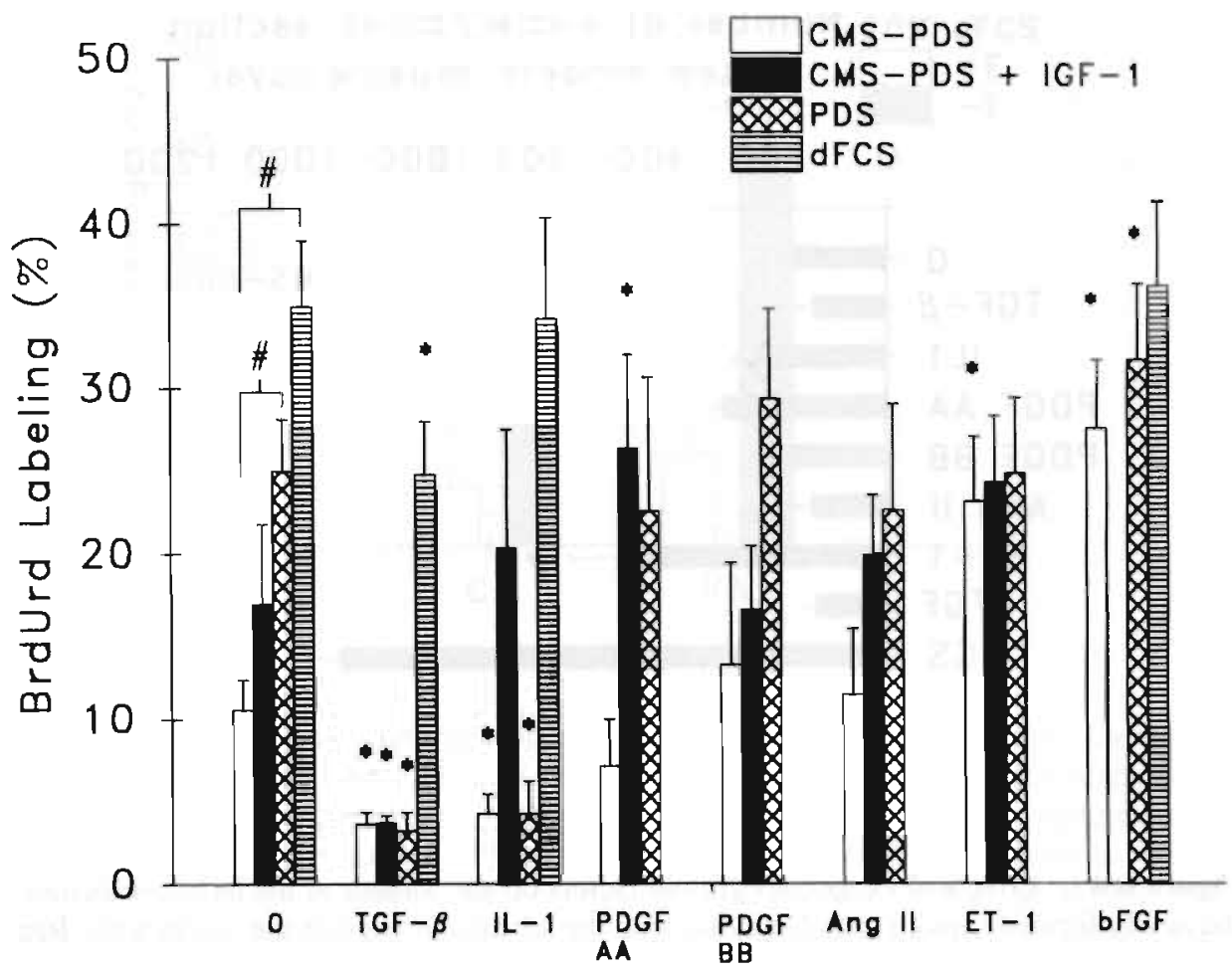

Figure 6.3 Effects of exogenous growth factors on DNA synthesis in the media of isolated and de-endothelialyzed renal arteries during 3 days of tissue culture. Nuclear incorporation of BrdUrd in the presence of 5\% CMS-PDS ( $\square$ ). 5\% CMS-PDS and IGF-1 $(100 \mathrm{nM})(\square), 5 \%$ PDS ( for TGF-B (10 ng/ml), IL-1 (100 U/ml), PDGF-AA (5 ng/ml), PDGF-BB $(5 \mathrm{ng} / \mathrm{ml})$, Ang II $(1 \mu \mathrm{M})$, ET $-1(10 \mathrm{nM})$ or bFGF $(10 \mathrm{ng} / \mathrm{ml})$. Data are expressed as \% labeling and are shown as means \pm SEM $(n=6)$. \# Differences from observations in the presence of CMSPDS are statistically significant $(p<0.05)$. The difference from observations in the absence of mediator is statistically significant $(p<0.05)$.

In the presence of $20 \%$ dFCS, which by itself, induced the strongest stimulation, TGF- $B$ but not IL-1 still reduced DNA synthesis. bFGF, the most powerful single growth factor used, did not enhance the stimulation of DNA synthesis obtained in the presence of dFCS (fig. 6.3). 


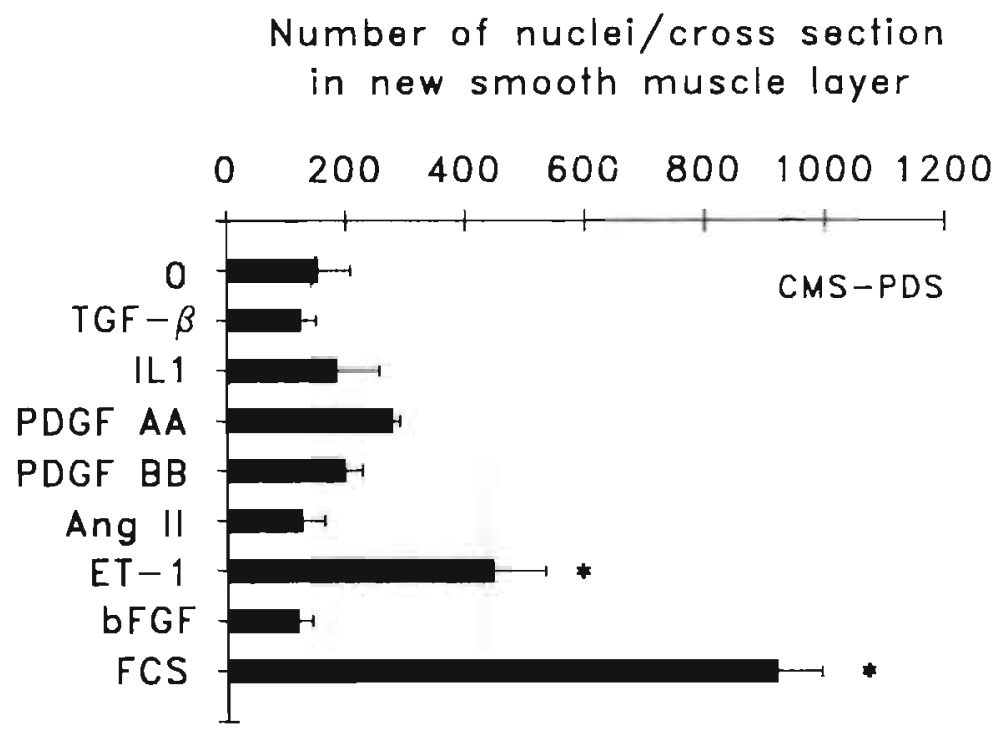

Figure 6.4 Effects of exogenous growth factors on the number of nuclei/cross section in the newly formed smooth muscle cell layer at the adventitial edge of the vessel wall. Renal arteries were isolated from adult WKY, denuded of endothelium and maintained in tissue culture for 4 days in $20 \% \mathrm{dFCS}$, directly followed by 14 days of culture in the presence of $5 \%$ CMS-PDS and $1 \mu \mathrm{g} / \mathrm{ml}$ indomethacin, supplemented with TGF- $B(10 \mathrm{ng} / \mathrm{ml})$, IL-1 $(100$ U/ml), PDGF-AA $(5 \mathrm{ng} / \mathrm{ml})$, PDGF-BB (5 ng/ml), Ang II ( $1 \mu \mathrm{M})$, ET-1 (10nM) or bFGF $(10 \mathrm{ng} / \mathrm{ml})$. dFCS was used as a positive control. Data are shown as means $\pm \operatorname{SEM}(n=6)$.

* The difference from observations in the absence of mediator is statistically significant $(\mathrm{p}<0.05)$.

\section{Exogenous Growth Factors and Cell Proliferation in Denuded Vessels}

To evaluate effects of high concentrations of recombinant growth factors on cell proliferation, denuded renal artery segments were exposed for 3 days to $20 \% \mathrm{dFCS}$ and subsequently for 14 days to CMS-PDS with or without growth factor. Also in these experiments which are summarized in figure 6.4, continuous exposure to $20 \% \mathrm{dFCS}$ was used as a positive control. TGF-B, IL-1 , PDGF AA, PDGF BB, Ang II and even bFGF did not stimulate cell proliferation in the periadventitial layer. ET-1, however, resulted in a significant increase in cell number. The effect of the peptide was, however, 


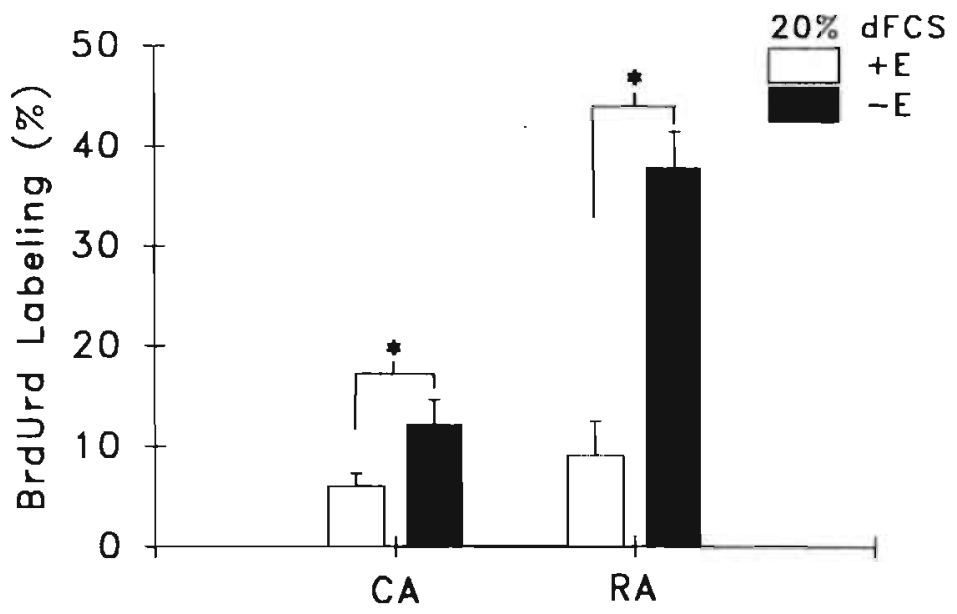

Figure 6.5 Effects of removal of the endothelium on BrdUrd labeling in the tunica media of carotid (CA) and renal (RA) arteries isolated from adult WKY. Arterial segments were either left intact or mechanically denuded of endothelium, and maintained in culture for 3 days in the continuous presence of $20 \% \mathrm{dFCS}$. Nuclear incorporation of BrdUrd is shown for segments with $(\square)$ and without $(\square)$ endothelium. Data are expressed as \% labeling and are shown as means \pm SEM $(n=6)$. All differences between carotid and renal arteries are statistically significant. ${ }^{*}$ Differences between preparations with and without endothelium are statistically significant $(\mathrm{p}<0.05)$.

smaller than that of dFCS.

\section{Effects of the endothelium}

In renal and carotid arteries that had not been denuded of endothelium the intramedial DNA synthesis that was stimulated by dFCS during short term culture was significantly smaller than in segments that had been de-endothelialized (fig. 6.5).

Conditioned medium collected from intact arterial segments, contained significantly more nitrite than medium conditioned by denuded vessels (fig. 6.6). Nitrite production by intact vessels was not reduced in arginine-free culture medium but was decreased by $200 \mu \mathrm{M}$ L-NAME (an inhibitor of NO synthase)(fig. 6.6). Furthermore, denuded vessels that were exposed to IL-1 produced more nitrite than denuded vessels maintained in the absence of the cytokine. Nitrite production stimulated by IL-1 required the presence of 


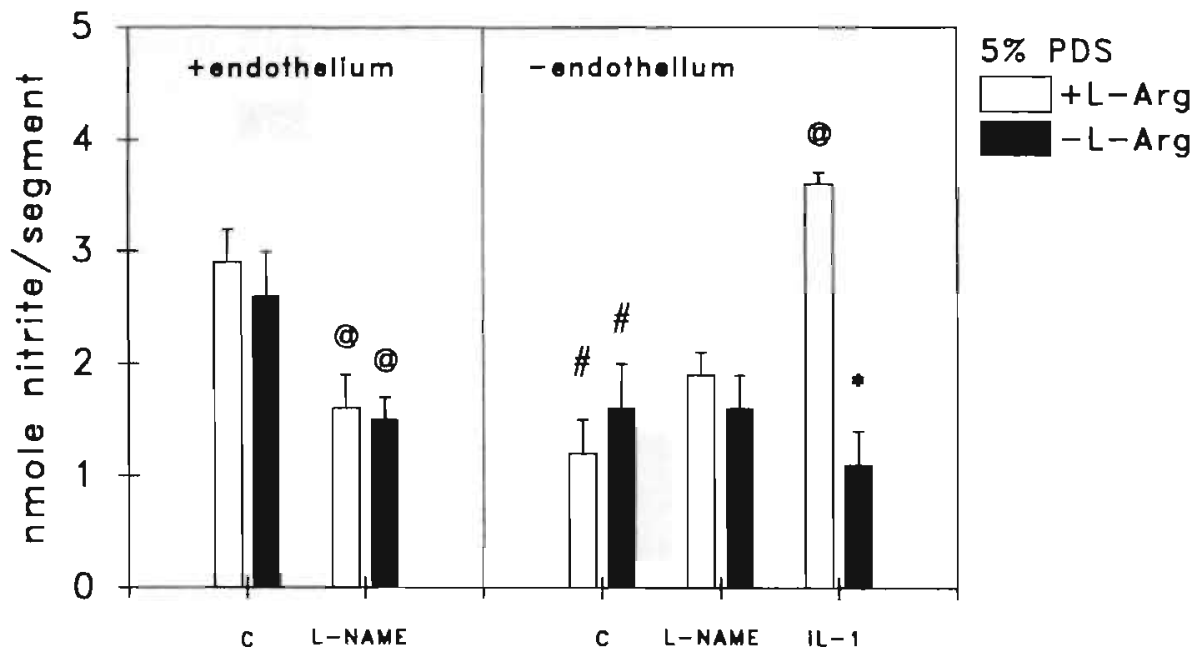

Figure 6.6 Effects of L-NAME, L-arginine-free medium and IL-1 on nitrite production, by isolated carotid artery segments (1/6 carotid artery). The vessels were either left intact (left) or denuded (right) of endothelium and maintained in culture during 3 days in the presence of $5 \%$ PDS. The experiments were performed in the absence ( $\square$ ) or presence ( $\square$ ) of L-arginine (L-Arg; $250 \mu \mathrm{M})$ with and without L-NAME $(200 \mu \mathrm{M})$ or IL-1 $(100 \mathrm{U} / \mathrm{ml})$. Data are shown as means \pm SEM $(n=6)$. * Differences from observations in the presence of L-Arg are statistically significant $(p<0.05)$. \# Differences from preparations with endothelium are statistically significant $(p<0.05)$. @ The difference from observations in drug-free controls is statistically significant $(p<0.05)$.

exogenous L-arginine (fig. 6.6). These findings suggest basal activity of endothelial NO synthase in intact vessels, and induction of NO synthase outside the endothelium by IL-1. Because IL- 1 can reduce medial DNA synthesis (fig. 6.3 ) they raise the possibility that also endothelial effects on intra-arterial DNA synthesis may be mediated by NO. However, differences between vessels with and without endothelium with respect to BrdUrd labeling, (i) persisted in the absence of exogenous L-arginine and were (ii) not affected by L-NAME (fig. 6.7).

Figure 6.8 illustrates effects and potential relationship to the endothelial influence for TGF- $B$ and $b F G F$, two factors that were shown above to modify medial DNA synthesis. Like the endothelium, exogenous TGF- $\beta$ could reduce the strong stimulation of DNA synthesis in medial cells by $20 \% \mathrm{dFCS}$. bFGF, on the other hand, blunted the inhibitory effect of the endothelium on the 


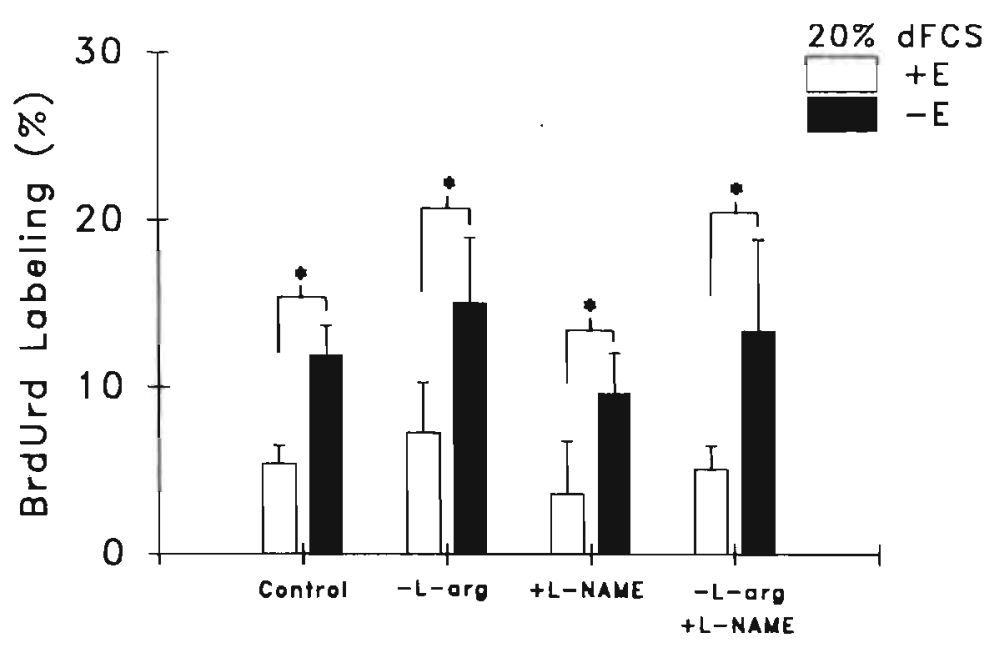

Figure 6.7 Effects of L-arginine-free medium and L-NAME on nuclear incorporation of BrdUrd in isolated arteries of adult WKY. Carotid arteries were either left intact ( $\square$ ) or mechanically denuded ( $\boldsymbol{(})$ of endothelium, and maintained in culture for 3 days in the presence of $20 \% \mathrm{dFCS}$. Nuclear incorporation of BrdUrd is shown, in the absence of Larginine (-L-arg), in the presence of L-NAME $(200 \mu \mathrm{M})$ and under both conditions (-L-arg $+\mathrm{L}$-NAME). Data were expressed as \% labeling and are shown a means $\pm \operatorname{SEM}(\mathrm{n}=6)$. * Differences between preparations with and without endothelium are statistically significant $(\mathrm{p}<0.05)$.

underlying smooth muscle cells (fig. 6.8).

\section{DISCUSSION}

The findings of the present study indicate that responses of arterial smooth muscle cells to sera and growth factors depend on their location within the arterial wall, the presence of other mitogens and on the endothelium.

In this as in earlier studies (Boonen et al, 1991; De Mey et al, 1991 ${ }^{\mathrm{C}}$ ), short term exposure of the isolated arterial wall to serum markedly stimulated medial DNA synthesis and long term culture in the presence of serum resulted in marked cellular proliferation. The number of medial cells was not altered but new cell layers developed at the adventitial edge of the preparations. Immunocytochemistry previously indicated that these periadventitial cells display smooth muscle-like properties (De Mey et al, $1991^{\mathrm{C}}$ ). We have proposed that 


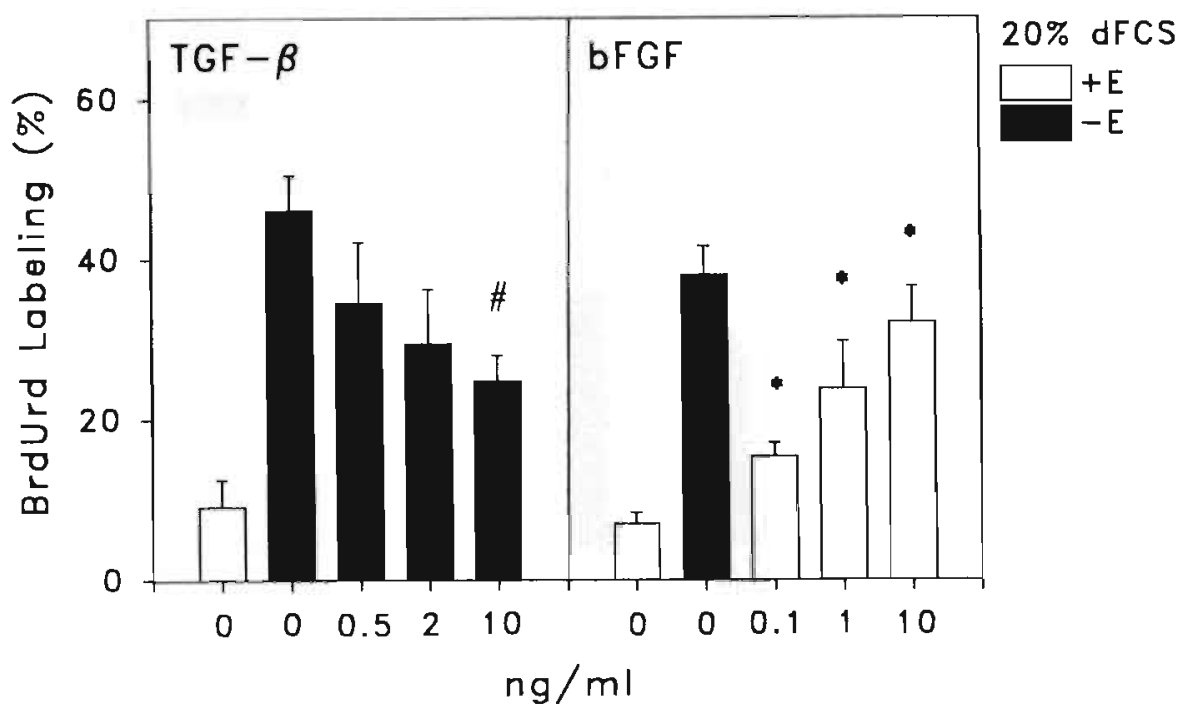

Figure 6.8 Effects of TGF- $B(0.5-10 \mathrm{ng} / \mathrm{ml})$ and $b F G F(0.1-10 \mathrm{ng} / \mathrm{ml})$ on medial DNA synthesis in isolated renal arteries from adult WKY rats. Arterial segments were either left intact $(\square)$ or mechanically denuded ( $\square$ ) of endothelium, and maintained in culture for 3 days in the presence of $20 \% \mathrm{dFCS}$. Data are shown as mean $\pm \mathrm{SEM}(\mathrm{n}=6)$. \# The difference from observations in denuded arteries in the absence of TGF- $B$ is statistically significant $(p<0.05) . *$ The difference from observations in intact arteries in the absence of bFGF is statistically significant $(p<0.05)$.

they are derived from smooth muscle cells that migrated out of the media. In general, the sequence of events during arterial organ culture, i.e transient stimulation of medial DNA synthesis, cell migration and proliferation outside the media, resembles the response of rat arteries to balloon catheter injury (Schwartz et al, 1986; Jackson and Schwartz, 1992). In the present study we confirmed that medial DNA synthesis can be stimulated to a larger extent in renal than carotid arteries (De Mey et al, $1991^{\mathrm{c}}$ ) and that in rat blood vessels the endothelium can reduce DNA synthesis in the underlaying muscle (De Mey et al, $1991^{\mathrm{a}}, 1991^{\mathrm{c}}$ ).

Medial DNA synthesis during short term culture and cellular proliferation during long term culture, differed in their serum dependency. In serumfree medium and in CMS-PDS a significant medial DNA synthesis was detected which exceeds the low levels of intra-arterial DNA synthesis normally observed in intact arteries in vivo (De Mey et al, 1991 ${ }^{\mathrm{d}}$; Schwartz et al, 1986; 
Clowes et al, 1989). The molecular origin of this response which may develop as a consequence of the injury that inevitably accompanies vessel isolation, remains unclear. However, neither serum-free medium nor CMS-PDS supported proliferation of periadventitial cells attracted by 4 day incubation in the presence of serum. Furthermore, effects of exogenous growth factors differed between the two systems and from what is known from cell culture experiments with dispersed arterial smooth muscle cells. All agents used have been described to stimulate cellular proliferation during cell culture under at least some conditions (Davies, 1986; Libby et al, 1988; Ross et al, 1986; Klagsbrun and Edelman, 1989; Bubin et al, 1989; Geisterfer et al, 1988). During short term arterial organ culture these "mitogens" enhanced, reduced or did not affect DNA synthesis. During long term arterial organ culture they hardly affected cellular proliferation. These discrepancies may be due to technical differences. Evaluation of medial DNA synthesis included experiments during mild stimulation elicited by CMS-PDS and PDS, which on the one hand may be suited to detect inhibitory influences and may, on the other hand synergies with mild mitogens. Differences between systems could therefore be quantitative rather than qualitative. Because we deliberately used concentrations of agents that in cell culture have been reported to be supramaximal (Davies, 1986; Libby et al, 1988; Ross et al, 1986; Klagsbrun and Edelman, 1989; Bubin et al, 1989; Geisterfer et al, 1988), this theoretical possibility is, however, unlikely. The most remarkable differences between the present organ culture experiments and cell culture are (i) the lack of stimulatory effect of PDGF on DNA synthesis and cellular proliferation (except for PDGF AA in the presence of IGF-1, see below), (ii) the inability of bFGF to promote extramedial cell proliferation and (iii) the inhibitory actions of IL-1 on DNA synthesis. PDGF's are powerful mitogens for isolated cells (Ross et al, 1986). In the arterial wall in vivo they, however, act primarily as chemotactic agents (Jawien et al, 1992). Also bFGF is a potent stimulus for isolated cells (Klagsbrun and Edelman, 1989). In vivo it participates in the stimulation of medial DNA synthesis following balloon catheter injury but not in the proliferation of neointimal cells (Olson et al, 1992). Our observation of a stimulatory effect of exogenous bFGF on medial DNA synthesis but not on proliferation of extramedial cells, suggests that not only temporal differences in the supply of bFGF but also differences in the responsiveness of medial and extramedial cells to the growth factor may be responsible for this phenomenon. In isolated human saphenous vein cells, IL-1 stimulates proliferation especially when prostaglandin synthesis is inhibited (Libby et al, 1988). We did not observe a stimulatory action of IL-1 in rat renal arteries despite blockade of cyclo-oxygenase. The cytokine rather reduced medial DNA synthesis in CMSPDS and PDS. Combined these observations indicate that findings in a given cell culture system can not be extrapolated to the arterial wall. Not only 
interspecies and regional differences have to be taken into account, but also differences in susceptibility to mediators between medial cells and extramedial cells. The molecular details of this heterogeneity remain obscure. A role for cell density, phenotype and extracellular matrix components merit attention in this respect (Thyberg et al, 1991). Because of its similarities with arterial responses to balloon catheter injury in vivo, further use of arterial organ culture in which cellular behavior can be studied in different environments in parallel, may be useful.

During the past few years it has become increasingly clear that several exogenously supplied mitogens do not act directly but rather through stimulation of the production of autocrine growth factors or their receptors. This has been documented repeatedly for PDGF and PDGF-receptors (Majesky et al, $1990^{\text {b}}$; Terracio et al, 1988). Consequently the inability of the arterial wall to respond to various growth factors may find its origin in the lack of responsiveness to this family of autocrine agents. Complex processes such as DNA synthesis and proliferation may on the other hand require the coordinated action of several factors (Sporn and Roberts, 1988). In this respect mitogens have been classified as competence and progression factors. IGF-1 belongs to this latter class. In the present study this factor unmasked significant stimulation of DNA synthesis by PDGF-AA and blunted the inhibitory action of IL-1. Future studies will thus have to concentrate on combinations of mitogens rather than on single factors. Furthermore, effects of mitogens on DNA synthesis and cellular proliferation may be obscured by enhanced synthesis of inhibitory agents such as prostaglandins and TGF-B (Libby et al, 1988; Morasaki et al, 1988; Owens et al, $1988^{\mathrm{b}}$ ). The former do not interfere with the present results because all experiments were performed in the presence of the cyclo-oxygenase inhibitor indomethacin. Other growth inhibitors can however not be excluded.

The most powerful modulator of medial DNA synthesis observed in the present study was the endothelium. This interpretation based on differences in vessels with and without endothelium, is not complicated by media damage that may accompany mechanical removal of endothelium. The technique used was previously found not to compromise the contractility of the wall (De Mey et al, $1991^{\mathrm{a}}$ ), a process that depends on the integrity of the smooth muscle cell membrane, their contractile filaments and the extracellular matrix. Furthermore, unlike balloon catheter injury (Schwartz et al, 1986; Jackson and Schwartz, 1992), our gentle mechanical removal of endothelium was accompanied by an insignificant $5 \%$ reduction of arterial DNA content (unpublished observations), which may represent removal of the innermost cell layer.

Cell culture experiments indicated that the endothelium can secrete all the factors that we tested during arterial organ culture (Warner et al, 1987b; Hannan et al, 1988; Vlodavsky et al, 1987; Bubin et al, 1989; Gryglewski et al, 1988; Moncada and Higgs, 1991). Of these, IL-1 and TGF- $B$ inhibited 
DNA synthesis while bFGF and ET1 stimulated the response. Thus as for its effects on media contractility, the endothelium has at least the potential to both inhibit and stimulate growth responses in underlaying smooth muscle. This conclusion was also reached when effects of endothelium removal in various arterial organ culture experiments were compared (De Mey et al, 1991a; Koo and Gotlieb, 1989).

In the present study the effects of IL-1 were used to verify the nature of the endothelial inhibition of medial DNA synthesis to some extent. This cytokine can stimulate expression of NO synthase in several cell types including smooth muscle cells (Moncada and Higgs, 1991). A NO synthase is normally constitutively expressed in endothelial cells (Moncada and Higgs, 1991) and its reaction product, NO, accounts for the majority of the effects of endothelium on arterial contractility (Furchgott and Vanhoutte, 1989). Similarity of effects of IL-1 and endothelium on medial DNA synthesis along with the observed production of nitrite, a stable degradation product of NO, are in line with a possible role of the radical in the endothelial modulation. However, differences between vessels with and without endothelium persisted in arginine-free culture medium and were not abolished by an arginineanalogue that inhibits NO synthase. In earlier experiments we observed that methylene blue, a scavenger of NO and an inhibitor of NO action on arterial contractility, did not stimulate DNA synthesis in rat arteries with endothelium (De Mey et al, $1991^{\mathrm{a}}$ ). In addition, while IL-1 displayed an inhibitory effect during moderate stimulation of DNA synthesis (i.e. in the presence of CMSPDS or PDS) the cytokine was ineffective in the presence of a high concentration of fetal calf serum. The endothelium, however, also markedly reduced the response to this strong stimulus. Combined the findings indicate that other phenomena than endothelium-derived NO and IL-1 participate in endothelial inhibition of medial DNA synthesis. A role for TGF- $B$ may be worth considering. In the present experiments, TGF- $B$ reduced DNA synthesis under various conditions. Furthermore, TGF- $\beta$ and $b F G F$ counteract one another in several systems (Davies 1986; Faller and Klagsbrun, 1988; Owens et al, 1988 ${ }^{\mathrm{b}}$; Sporn and Roberts, 1988) and we found that bFGF can potently inhibit the endothelial effect on medial DNA synthesis. Clearly additional experiments are required to firmly establish the role of TGF-B.

In summary, factors that may be produced by smooth muscle and endothelial cells were found to affect DNA synthesis and cellular proliferation in isolated arteries. Both stimuli and inhibitors were identified. They affected medial DNA synthesis and extramedial cell proliferation differently. It was furthermore confirmed that the endothelium can markedly suppress stimulated DNA synthesis in rat arteries. It is unlikely that this is primarily due to endothelium-derived prostaglandins, NO or IL-1. 



\section{Chapter}

\section{In Vivo DNA synthesis is Not Uniformly Increased in Arterial Smooth Muscle of Young Spontaneously Hypertensive Rats}

J.G.R. De Mey, M.J.A.P. Daemen ${ }^{a}$, H.C.M. Boonen, F.T. Bosman ${ }^{a}$, E. H. Dijkstra, G.E. Fazzi, G.M.J. Janssen, P.M.H. Schiffers, H.A.J. Struijker-Boudier and M.J.J.F Vrijdag

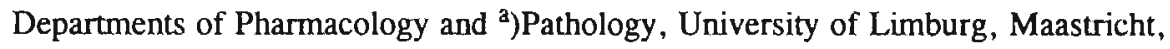
the Netherlands.

(Journal of Hypertension 1991, 9: 695-701) 


\section{INTRODUCTION}

The arterial system of human essential hypertensives and that of spontaneously hypertensive rats (SHR) is altered in several aspects (Folkow, 1982). Larger vessels ( $>100 \mu \mathrm{m}$ diameter) display an increased wall:lumen ratio. This can result from both a reduction in vessel diameter and media hypertrophy (Mulvany et al, 1978; Baumbach and Heistad, 1989; LeNoble et al, 1990). In conduit arteries, the latter is primarily due to hypertrophy of the arterial smooth muscle cells (Owens and Schwartz, 1982; Schwartz et al, 1986; Owens, 1989). In resistance-sized arteries, on the other hand, hyperplasii rather than cellular hypertrophy or hyperploidy has been observed (Mulvany et al, 1978, 1985; Lee, 1985; Owens et al, 1988; Black et al, 1988). Hyperplastic and hypertrophic arterial changes could differ in susceptibility to correction by antihypertensive therapy. It is not clear, however, what mechanisms underlie hyperplastic and cellular hypertrophic arterial growth and what is responsible for their heterogeneous distribution over the arterial tree. Different stimuli could be involved (Owens, 1989) and they could represent primary and secondary adaptive changes, respectively (Folkow, 1982; Lec and Smeda, 1985). In line with this suggestion is a recent study by Yang et al (1989) in which elevated DNA synthesis was observed in small, but not large, mesenteric arteries of 1-week-old SHR.

To strengthen the possibility that a temporal difference could underlie the hyperplastic and hypertrophic changes in small and large arteries of SHR, we muasured DNA synthesis in elastic, muscular and resistance arteries of 6-weck-old normotensive Wistar-Kyoto rats (WKY) and SHR in situ and compared our observations to those of Yang et al (1989). To evaluate consequences of this DNA synthesis, we estimated, for a limited number of large arteries, the number of arterial smooth muscle cells, determined arterial DNA content and compared them for 6- and 20-week-old WKY rats and SHR. These ages were selected because: (1) at 6 weeks rats still grow significantly; (2) blood pressure rises sharply between 5 and 7 weeks of age in SHR (Evenwel et al, 1983); and (3) hypertension is fully established in 20 -week old SHR.

\section{MATERIALS AND METHODS}

The experiments were performed in 6- and 20-week-old male SHR (Evenwel et al, 1983; Okamoto and Aoki, 1963) and normotensive WKY rats. Rats of both strains were obtained from local inbred colonies (University of Limburg, Maastricht, the Netherlands). Body weight, heart rate and systolic blood pressure, as determined by tail-cuff plethysmography (Narco 
Biosystems, Houston, Texas, USA), are summarized in table 1.

To record intra-arterial DNA synthesis in vivo, eight 6-week-old SHR and WKY rats were equipped under ether anesthesia with an osmotic minipump (Alzet model 2001, Alza, Palo Alto, California, USA) continuously delivering the thymidine-analogue 5-bromo-2'-deoxyuridine (BrdUrd; Sigma Chemicals, St Louis, Missouri, USA) at $1 \mu \mathrm{mol} / \mathrm{kg}$ per h, subcutaneously. Forty-eight hours later, their vascular system was perfusion fixed with $4 \%$ phosphate-buffered formaldehyde at a pressure of $100 \mathrm{mmHg}$. The thoracic aorta and superior mesenteric artery as well as the left carotid, renal and femoral arteries and mesenteric and renal resistance-sized arteries were isolated. The small mesenteric vessels (diameter in situ, 150-200 $\mu \mathrm{m}$ ) corresponded to fourth order side branches of the superior mesenteric artery and were isolated from the pinned out mesentery of a small intestinal loop. The small renal vessels were interlobar arteries that were dissected out of longitudinal kidney slices. From each animal, two specimens from both types of resistance artery were sampled. All arterial preparations that were harvested were postfixed in formaldehyde for 12 to $24 \mathrm{~h}$, embedded in paraffin, sectioned ( $4 \mu \mathrm{m}$ cross sections) and processed for histology and immunohistochemistry.

All arterial specimens were sectioned according to the following semiserial protocol. At six levels separated by $60 \mu \mathrm{m}$, four cross sections were obtained. One section from each level was stained with hematoxylin and eosin. The other sections were stained immunohistochemically for BrdUrd (Gratzner, 1982). We therefore used a monoclonal antibody against the thymidine analogue (Eurodiagnostics, Apeldoorn, the Netherlands) and peroxydase-conjungated second-step reagents. In all cases, the immunoreactivity was limited to the nuclei. In negative controls, i.e. sections not exposed to the primary antibody or sections from vessels that had not been exposed to BrdUrd, no staining was observed. To quantify the incorporation of the thymidine analogue, the percentage of labeled nuclei was determined according to the following formula:

\section{BrdUrd L\% $=100 *$ (number of labeled nuclei/total number of nuclei)}

where BrdUrd $\mathrm{L} \%$ is the BrdUrd labeling percentage. The analysis were limited to the muscular media layer of the arterial preparations except for the thoracic aorta in which nuclear incorporation of BrdUrd was also quantified in the endothelium. The sensitivity of this approach is influenced by the number of nuclei that can be counted and thus differed dramatically between large and small specimens. In none of the large arteries, however, was the difference between the number of labeled nuclei in sections obtained at the same level or that between BrdUrd L\% at the different levels statisti- 
cally significant. We therefore pooled the data obtained at the different levels of an individual arterial segment and calculated BrdUrd L\% on the basis of these sums. The accuracy of BrdUrd L\% then ranged from $0.05 \%$ in resistance arteries and aortic endothelium to $0.002 \%$ in aortic smooth muscle. The observations were at least 40 -fold larger than these detection limits.

From additional 6- and 20-week-old rats that were killed by a sharp blow on the back of the head and exsanguination, the left carotid artery and thoracic aorta were harvested. These were mechanically denuded of endothelium, weighed, frozen in liquid nitrogen and stored at $-70^{\circ} \mathrm{C}$. Their DNA content was determined as described by Labarca and Paigen (1980). Thawed tissues were solubilized in $1 \mathrm{~N} \mathrm{NaOH}$ for $24 \mathrm{~h}$. Aliquots of the extract were then neutralized $(\mathrm{pH} \mathrm{7.7)}$ and their DNA content was measured fluorimetrically using the fluorochrome, bisbenzimidazole (Hoechst 33258; France Biochem, Bierges, Belgium) and calf thymus DNA (Sigma) as a standard. Ribonucleic acid does not interfere in this assay, which can detect as little as 10 ng DNA (Labarca and Paigen, 1980).

To further analysis possible structural consequences of intra-arterial DNA synthesis in young rats, we counted the number of nuclear profiles in media cross-sections of arteries from 6- and 20-week-old rats. This estimate of the number of smooth muscle nuclei in a defined unit of vessel length depends on the nuclear dimensions (Mulvany et al, 1985). These were not compared between the different experimental groups in the present study. Conclusions based on the number of medial nuclear profiles were, however, supported by measurements of arterial DNA content per unit vessel length. Carotid arteries of WKY rats contained 0.66 (95\% confidence interval, $0.64-0.68)$ and $0.57(0.52-0.62) \mu \mathrm{g} D N A / m m$ and those of SHR, 0.69 $(0.67-0.71)$ and $0.70(0.65-0.75) \mu \mathrm{g}$ DNA/mm at 6 and 20 weeks, respectively. Thoracic aortas of WKY rats contained $2.3(2.1-2.5)$ and $1.7(0.8-2.6)$ $\mu \mathrm{g}$ DNA/mm and those of SHR $2.3(2.1-2.5)$ and $1.9(1.7-2.1) \mu \mathrm{g}$ DNA$/ \mathrm{mm}$ at 6 and 20 weeks, respectively.

Statistical analysis of the data included the following comparisons: between arterial types of the same animals, between arterial types of the same strain and between strains. Analysis of variance is suited for these comparisons provided that the data are normally distributed and variances are comparable (Sokal and Rohlf, 1981). This was the case for data on weight, blood pressure, arterial cell number and arterial DNA content. In several SHR arteries, BrdUrd L\% were, however, not normally distributed and their variances differed significantly from those in WKY arteries (Bartlett's X ${ }^{2}$ test). A Box-Cox procedure (Sokal and Rohlf, 1981) indicated that this could be remedied by logarithmic transformation of the data. As a consequence, all BrdUrd L\% data were transformed and compared by 
Table 7.1 Properties of young and adult rats.

\begin{tabular}{lcccc}
\hline & \multicolumn{2}{c}{6 weeks } & \multicolumn{2}{c}{20 weeks } \\
& WKY & SHR & WKY & SHR \\
\hline Body weight (g) & 125 & 123 & 318 & 309 \\
& $(111-139)$ & $(114-132)$ & $(293-343)$ & $(279-339)$ \\
& & & & 136 \\
Systolic blood \\
$\begin{array}{l}\text { blood pressure } \\
\text { (mmHg) }\end{array}$ & $(82-92)$ & $(94-106)$ & $(120-152)$ & $(176-222)$ \\
& & & & \\
$\begin{array}{l}\text { Heart rate } \\
\text { (beats/min) }\end{array}$ & 400 & 424 & 325 & 373 \\
& $(347-453)$ & $(357-491)$ & $(260-390)$ & $(333-412)$ \\
\hline
\end{tabular}

Data are shown as means (95\% confidence intervals), $n=8 . * P<0.05$, versus age-matched Wistar-Kyoto rats (WKY). SHR, spontaneously hypertensive rats.

analysis of variance. This was followed by Scheffe's t-test for multiple comparisons. All conclusions reached by this procedure with respect to differences in BrdUrd L\% between types of artery of WKY rats and SHR were confirmed by confidence interval tests on the difference between means of transformed data and by non-parametric Wilcoxon's signed-rank tests on untransformed data (Sokal and Rohlf, 1981). All data are shown as means of untransformed or retransformed data with $95 \%$ confidence intervals. These are asymmetric in the case of antilogs of log transformed observations. $\mathrm{P}<0.05$ was selected as the level of significance throughout.

\section{RESULTS}

Systolic blood pressure was significantly higher in 6-week-old SHR than in age-matched WKY rats (table 7.1). Body weight and heart rate did not differ significantly between 6-week-old rats of both strains. Development from 6 to 20 weeks of age increased blood pressure to a larger extent in SHR than WKY rats (table 7.1).

Figure 7.1 summarizes relative extents of nuclear incorporation of BrdUrd in the media of elastic, muscular and resistance arteries of 6-week-old 


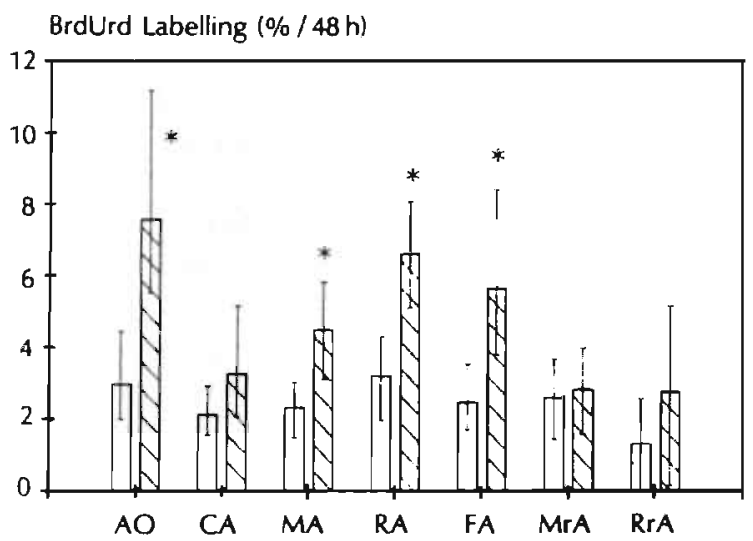

Figure 7.1 Extent of in vivo DNA synthesis in the media of various arteries of 6-weekold Wistar-Kyoto $(\square)$ and spontaneously hypertensive rats ( Rats were subcutaneously infused with $1 \mu \mathrm{mol} / \mathrm{kg}$ per h 5-bromo-2'-deoxyuridine (BrdUrd) during $48 \mathrm{~h}$. Following fixation of their arterial system, the thoracic aorta (AO), the carotid (CA), superior mesenteric (MA), renal (RA) and femoral (FA), as well as mesenteric (MrA) and renal $(\operatorname{RrA})$ resistance-sized arteries were isolated. The percentage of labeled medial nuclei was determined by immunohistochemistry on cross-sections. Data are shown as retransformed means with $95 \%$ confidence intervals. Ninety-five per cent confidence intervals for interstrain differences between means of log transformed data were: (0.204-0.634) in AO; $(-0.049-0.423)$ in CA; $(0.087-0.487)$ in MA; $(0.122-0.512)$ in RA; $(0.158-0.508)$ in FA; ($0.055-0.129)$ in $\mathrm{MrA}$; and $(-0.080-0.720)$ in $\operatorname{RrA} .{ }^{*} \mathrm{p}<0.05$, versus Wistar-Kyoto rats (analysis of variance followed by Scheffe's t-test on log transformed data).

SHR and WKY rats following $48 \mathrm{~h}$ continuous exposure to the thymidine analogue in vivo. In WKY rats, this index of DNA synthesis was uniformly distributed over the arterial system, i.e. differences between types of large artery and between large and resistance arteries were not statistically significant. In addition, the variability between individuals was significantly larger than that between arterial types (Friedmans method for randomized blocks). Thus, when the labeling was relatively high in the carotid artery of a given animal, this was also the case in, for instance, the femoral artery of the same animal.

In 6-week-old SHR, nuclear incorporation of BrdUrd in the arterial media differed between different segments of the arterial system (fig. 7.1). The difference between the observations in WKY rats and SHR were statistically significant in the aorta, superior mesenteric artery, renal artery and femoral 
Table 7.2 Number of medial nuclear profiles in cross sections of elastic and muscular arteries of young and adult rats.

$$
6 \text { weeks }
$$

20 weeks

\begin{tabular}{lcccc} 
& WKY & SHR & WKY & SHR \\
\hline Thoracic aorta & 1523 & $1902^{*}$ & $997^{*}$ & ND \\
& $(1380-1666)$ & $(1707-2095)$ & $(846-1148)$ & \\
Carotid artery & 375 & 408 & 309 & 355 \\
& $(315-435)$ & $(332-484)$ & $(256-362)$ & $(302-408)$ \\
Mesenteric artery & 408 & $350^{*}$ & 378 & ND \\
& $(391-425)$ & $(320-380)$ & $(326-429)$ & \\
Renal artery & 187 & 166 & 170 & 174 \\
& $(164-210)$ & $(149-183)$ & $(130-210)$ & $(157-191)$ \\
Femoral artery & 239 & $282^{*}$ & 237 & ND \\
& $(193-285)$ & $(248-316)$ & $(209-265)$ & \\
\hline
\end{tabular}

Data are shown as means (95\% confidence intervals) of the average observations in 6-14 sections/vessel of 7-18 individuals. ${ }^{*} \mathrm{P}<0.05$, versus young Wistar-Kyoto rats (WKY). ND, not determined; SHR, spontaneously hypertensive rats.

artery but not in the carotid artery, mesenteric or renal resistance arteries (fig. 7.1). The data presented in figure 7.1 indicate that observations in renal resistance arteries of SHR, unlike those in mesenteric resistance arteries, tended to be larger than in comparable vessels of WKY rats. This did not, however, reach statistical significance.

In WKY aorta, BrdUrd labeling in the endothelium, 4.79 (3.35$6.82) \% / 48 \mathrm{~h}$, did not significantly differ from that in the media, 2.99 (2.004.47)\%/48 h. In SHR aorta, endothelial labeling, 3.99 (3.06-5.20)\%/48 h, averaged only $50 \%$ of that in the media, $7.85(5.53-11.17) \% / 48 \mathrm{~h}$, and did not exceed nuclear incorporation of BrdUrd in WKY aortic endothelium.

In table 7.2, the number of nuclear profiles in media cross-sections from arteries of 6-week-old rats are compared with those observed in arteries of 20week-old rats. In neither type of artery was this number significantly lower in young than in adult rats.

Figure 7.2 summarizes weight and DNA content of carotid arteries and 

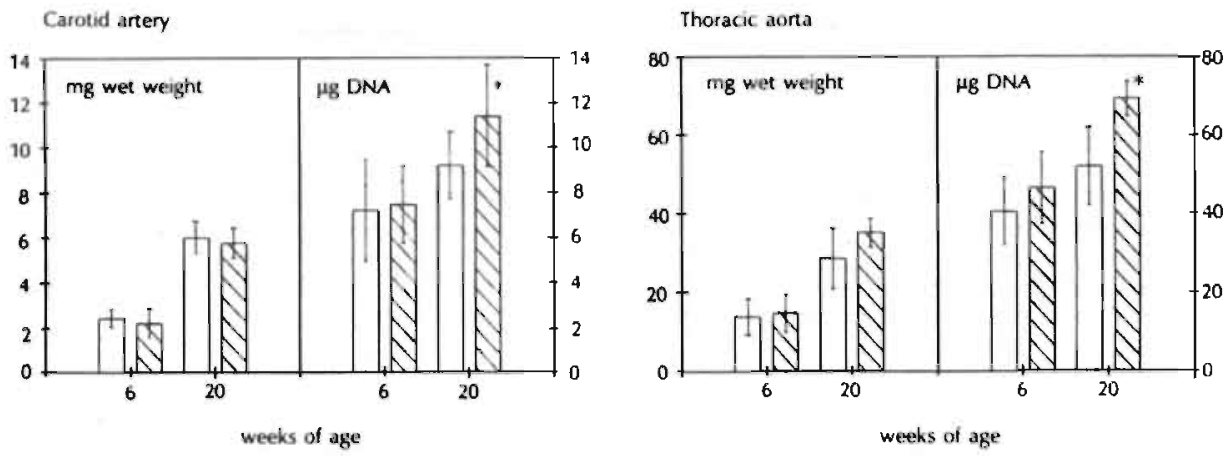

Figure 7.2 Wet weight and DNA content of the left main carotid artery and the thoracic aorta of 6- and 20-week-old Wistar-Kyoto rats $(\square)$ and spontaneously hypertensive rats ( $\mathbb{N}$ ). Data are shown as means with $95 \%$ confidence intervals. ${ }^{*} p<0.05$, versus matched WistarKyoto rats.

thoracic aortas of 6- and 20-week-old rats. DNA content did not differ between vessels of young WKY rats and young SHR. It was, however, significantly larger in carotid arteries and aortas of adult SHR than in those of adult WKY rats. With maturation from 6 to 20 weeks of age, carotid arteries gained 148 and $159 \%$ weight and 28 and $53 \%$ DNA in WKY rats and SHR, respectively. Increases in aortic weight and DNA content averaged 106 and $138 \%$, and 28 and $49 \%$ for WKY rats and SHR, respectively. The increases of DNA content in SHR carotid artery, $3.92(3.01-4.83) \mu \mathrm{g}$, and SHR thoracic aorta, 22.7 (15.9-29.5) $\mu \mathrm{g}$, were significantly larger than in those in WKY vessels, which averaged $1.98(1.09-2.87) \mu \mathrm{g}$ and $11.4(6.95-15.8) \mu \mathrm{g}$ for carotid artery and aorta respectively.

\section{DISCUSSION}

We recorded DNA synthesis in the media of large and small arteries of young WKY rats and age-matched SHR in which blood pressure was moderately elevated. In WKY rats, the relative extent of DNA synthesis was uniform over the arterial tree. In SHR, intra-arterial DNA synthesis was elevated in some large arteries but not in resistance arteries.

DNA synthesis is involved in both hyperplastic and hypertrophic growth 
of arterial smooth muscle because DNA synthesis is an essential preparative step for mitosis and because hypertrophic arterial smooth muscle cells have been observed to display polyploidy (Owens and Schwartz, 1982; Schwartz et al, 1986; Owens, 1989; Schwartz, 1984). DNA synthesis is routinely estimated by autoradiography of ${ }^{3} \mathrm{H}$-thymidine incorporated in nuclei during the $\mathrm{S}$-phase of the cell cycle. Gratzner (1982) presented the thymidine analogue BrdUrd as a valid cold alternative that can be traced with a monoclonal antibody. Comparative studies in various tissues (Sugihara et al, 1986; Van Furth and Van Zwet, 1988), including blood vessels (Daemen et al, 1989), indicated that the two approaches yield identical results. The accuracy of either technique depends on the number of cells that are in S-phase at a given point in time, the time during which the label is applied and the number of nuclei that one is willing or able to count. The latter can be limiting in minute tissue samples such as resistance-sized arteries. We attempted to circumvent this by applying the label for a relative long period of time and by performing the analysis in young animals that are growing at a significant rate (6-week-old rats gain at least $5 \%$ body weight/day). It should be taken into account, however, that labeling periods that are longer than the transit time from $S$-phase to karyokinesis will overestimate the proportion of cells that occupied S-phase during labeling.

Significant DNA synthesis was observed in arterial smooth muscle of 6week-old WKY rats. On a relative basis, it was uniformly distributed over a variety of elastic, muscular and resistance-sized arteries ( $2.5 \%$ of all arterial smooth muscle nuclei incorporated BrdUrd during the 2-day labeling period). This is intermediate between the thymidine labeling that has been reported for large arterial smooth muscle in neonatal and adult normotensive rats (Yang et al, 1989; Clowes et al $^{\mathrm{a}}$, 1983; Reidy, 1990). It has previously been suggested that, in young rats, the arterial smooth muscle is not yet fully differentiated and partly composed of rapidly proliferating muscle cells (Schwartz et al, 1986; Skalli et al, 1986). Since mean arterial pressure, pulse pressure, flow, shear stress and wall stress vary dramatically over the arterial system (Gow, 1980), the observed uniform distribution of DNA synthesis in elastic, muscular and resistance arterial smooth muscle of 6-week-old WKY rats indicates that these physical forces do not govern the growth of the arterial media in young normotensive rats.

In 6-week-old SHR, unlike WKY rats, DNA synthesis was more pronounced in large arteries than in resistance arteries. DNA synthesis was, in addition, larger in some large SHR arteries than in large WKY arteries. There was, however, no difference between mesenteric or renal resistance arteries of both strains. These findings in young rats differ from those in newborn and adult rats. Yang et al (1989) reported increased ${ }^{3} \mathrm{H}$-thymidine labeling in small mesenteric arteries of 1-week-old SHR. They did not, however, observe 
differences between superior mesenteric arteries of 1-week-old SHR and WKY rats or between large and small mesenteric vessels of 2- and 4-week-old SHR and WKY rats. Loeb and Bean (1986), on the other hand, observed a fivefold elevation of DNA synthesis in aortas of 17-week-old SHR, but no difference between aortas of 4- or 21-week-old SHR and WKY rats. Taken together, this suggests that the elevated DNA synthesis in large arteries of 6-week-old SHR which we observed represents a secondary adaptive response to the marginally elevated blood pressure. This could be limited to the large vessels in which the increase in pressure is, at least in absolute terms, more marked than in resistance arteries. Aortic DNA synthesis is increased in experimental models of secondary hypertension and this can be prevented by various antihypertensive therapies (Schwartz et al, 1986; Owens, 1989; Schwartz, 1984). These observations strengthen the secondary adaptive nature of elevated DNA synthesis in large arteries of young SHR. This conclusion concerning SHR in vivo is, however, in contrast to other findings: (1) elevation of aortic DNA synthesis precedes the rise of blood pressure in renal hypertensive rats (Reidy, 1990); (2) cytosine arabinoside, an inhibitor of DNA synthesis, delays twokidney, one clip renal hypertension (Loeb and Bean, 1986); and (3) aortic smooth muscle cells isolated from adult SHR proliferate faster than WKY during cell culture (Kanbe et al, 1983).

The observations of the present study indicate regional heterogeneity of the intra-arterial DNA response to hypertension. In conjunction with those of Yang et al (1989) and Loeb and Bean (1986), they also suggest a temporal heterogeneity of increased DNA synthesis in large and small arteries of SHR. Collectively, the observations in neonatal young and adult rats suggest that a transient elevation of intra-arterial DNA synthesis originates at the microcirculatory level during the early stage of the development of SHR and then slowly progresses upstream. Also in this progression, there could be a role for elevated intravascular pressure (Joshua, 1984).

The mechanisms that underlie regional and temporal differences in intraarterial DNA synthesis remain unclear. Since no elevation of DNA synthesis was observed in aortic endothelium of young SHR, the mechanisms are, however, selective for smooth muscle and do not include responses to endothelial injury (Schwartz et al, 1986; Schwartz, 1984)

Maturation from 6 to 20 weeks of age in a variety of large arteries of WKY rats was not accompanied by an increase in the number of arterial smooth muscle nuclear profiles per cross-section. During this period, the weight of carotid arteries and aortas more than doubled and their DNA content increased by $25 \%$. Large arterial growth during later stages of development thus consists primarily of an increase in mass and, to a smaller extent, an increase in longitudinal cell number and possibly in nuclear DNA content (Schwartz et al, 1986; Owens, 1989). The lack of an increase in the number 
of nuclear profiles per media cross-section is of interest. Also, more drastic mitogenic interventions such as mechanical arterial injury (Clowes et al, $1983^{\mathrm{a}}$ ), acute hypertension (Schwartz et al, 1986; Owens, 1982;

Schwartz, 1984) and serum mitogens (Boonen et al, 1991) fail to increase the number of medial nuclei per arterial cross-section. In addition, most arteries appear to have developed their adult number of layers of smooth muscle cells before birth (Wolinsky and Glagow, 1967). This strongly suggests that, in the adult arterial media, the structural consequences of even more powerful mitogenic conditions are counteracted. The mechanisms of this suspected intramedial growth inhibition are not yet clear. However, the recent finding that antihypertensive therapy with inhibitors of angiotensin converting enzyme reverses polyploidy in the aorta of adult SHR (Black et al, 1989) stresses the dynamic nature of such an equilibrium. In view of this, the early elevation of DNA synthesis in SHR resistance arteries (Yang et al, 1989) could result in hyperplasia (Mulvany et al, 1978, 1985; Lee, 1985; Owens et al, 1988) because of immaturity of intramedial growth control. Arterial smooth muscle cell hypertrophy and hyperploidy, which are cellular changes that occur normally on the way to karyokinesis (Schwartz et al, 1986; Owens, 1989), could occur in large arteries at a later developmental stage as a result of two opposing growth-affecting forces. Whether, in addition, different mitogenic stimuli are involved in early and late developmental stages of genetic hypertension (Owens, 1989) or whether the same stimuli are operational in small and large vessels at different times remains to be established.

We observed uniform DNA synthesis in elastic, muscular and resistancesized arteries of 6-week-old WKY rats. This questions the importance of blood pressure as a driving force for arterial growth during later stages of development at least in normotensive rats. In 6-week-old SHR, DNA synthesis was elevated in some large arteries but not in resistance arteries. We speculate that, in addition to this regional heterogeneity, there is also a temporal component. Taken together, our observations suggest that the hyperplastic nature of SHR resistance arteries results from a site-selective mitogenic response at the early developmental stage at which the adult number of arterial smooth muscle cells is not yet reached. 



\section{Chapter}

8

Heterogeneity of Growth Responses in the Arterial System of Young Rats during Vasoconstrictor Infusion

J.G.R. De Mey, P.M.H. Schiffers, M.J.A.P. Daemen ${ }^{2}$, E.M. van Kleef $^{\mathrm{a}}$, R. Houben, G.E. Fazzi and D. van Ingen Schenau

Departments of Pharmacology and ${ }^{\text {a)}}$ Pathology, University of Limburg, Maastricht, the Netherlands. 


\section{INTRODUCTION}

In human and experimental hypertension, arterial wall thickness is increased (Folkow, 1982). This hypertrophy contributes to elevated peripheral vascular resistance and may be an independent risk factor for secondary cardiovascular diseases. In large arteries, it is primarily due to hypertrophy/hyperploidy of the arterial smooth muscle cells (ASMC) (Schwartz et al, 1986; Owens, 1989). Small resistance-sized arteries, on the other hand, display ASMC hyperplasia and remodeling (i.e., organization of the media around a narrower lumen) (Mulvany et al, 1985; Owens et al, 1988 ${ }^{\mathrm{a}}$ ). Analysis of the origin of this regionality may allow to better define targets for rational cardiovascular drug therapy. Regional heterogeneity of arterial structural changes may result from non-uniform input of mitogenic stimuli along the vascular tree (Owens, 1989; Bevan and Tsuru, 1981; Head, 1991). A large body of evidence indicates that the renin-angiotensin system and sympathetic nerves are involved in the development of hypertension and of the arterial structural changes that are associated with it (Head, 1991; Lee and Smeda, 1985; Sahlgren et al, 1986; Lever, 1986). The density of sympathetic nerves varies considerably along the arterial tree (Abboud, 1982). This may participate in regionality of structural responses to both sympathetic stimulation and angiotensin II (Ang II), because the peptide increases sympathetic nerve activity centrally and peripherally (Severs and Daniels-Severs, 1973; Zimmerman, 1978) and potentiates ASMC responses to alpha-adrenoceptor stimulation (Vanhoutte et al, 1981). Part of the structural effects of neurohumoral agents may be indirect and mediated through elevation of arterial wall stress. On the other hand, experimental approaches of mechanisms of atherogenesis suggest that the responsiveness of ASMC to mitogens is not uniform. It may not only depend on the phenotype of the ASMC but may also differ between subtypes of ASMC (Schwartz et al, 1986; Chamley-Campbell et al, 1979, 1981; Blank et al, 1988; Skalli, 1984). This has not been considered yet with respect to arterial structural changes in hypertension.

In the present study, we infused Ang II or the alpha-1 adrenergic agonist phenylephrine (PHE) in young rats. We documented the distribution of indices of arterial growth responses by comparing different types of blood vessels. Furthermore, with the use of the phenotypic marker desmin, we evaluated whether growth responses in the aorta were restricted to a subset of smooth muscle cells. Indices of growth responses included changes in media crosssectional area, DNA synthesis and expression of cyclin, a proliferating cell nuclear antigen (PCNA). The experiments were performed in 6-week old Wistar-Kyoto rats (WKY), using genetically hypertensive rats of the Okamoto strain (SHR) for comparison. At 6 weeks of age, basal intra-arterial DNA synthesis is still significant in WKY and hypertension becomes statistically 
significant in SHR.

\section{MATERIALS AND METHODS}

\section{Animals}

Six-week old Wistar-Kyoto rats (WKY) and age-matched spontaneously hypertensive rats (SHR) were obtained from local inbred strains (University of Limburg, Maastricht, The Netherlands). Four groups of 8 rats each were defined. Three groups consisted of WKY that were infused during 4 days with either saline (WKY), $2.0 \mathrm{mg} / \mathrm{kg} /$ day PHE (Sigma Chemicals, St. Louis, MO, USA) or $0.3 \mathrm{mg} / \mathrm{kg} / \mathrm{day}$ Ang II (Sigma Chemicals), through a subcutaneously implanted osmotic minipump (Alzet, model 2001, Alza, Palo Alto, CA, USA). The last group consisted of SHR that received saline. Prior to implantation and after 4 days of treatment, systolic blood pressure (SBP) and heart rate (HR) were measured by tail-cuff plethysmography (Narco Biosystems, Houston, TX, USA). Four days after implantation of the minipumps, the rats were sacrificed and the thoracic aorta, carotid, superior mesenteric, renal, and femoral arteries as well as mesenteric and intrarenal resistance-sized arteries were isolated. Heart and kidneys were also dissected.

\section{Immunohis.ochemistry and morphometry}

To estimate DNA synthesis, the animals were equipped with a second osmotic minipump, releasing the thymidine analogue 5-bromo-2'-deoxyuridine (BrdUrd) (Gratzner. 1982) at $5.5 \mathrm{mg} / \mathrm{kg} /$ day. The isolated vessels and organs were embedded in paraffin and cross-sections of $4 \mu \mathrm{m}$ were stained with hematoxylin and eosin for evaluation of overall structure. We used a monoclonal antibody (Eurodiagnostics, Apeldoorn, The Netherlands) and an indirect enzyme labeled antibody technique with a peroxidase-conjugated second step to visualize nuclei that had incorporated the thymidine analogue (Gratzner, 1982). To quantify the incorporation of BrdUrd, the percentage of nuclei that stained for peroxidase was determined for the tunica media. Similar immunohistochemical techniques were used to evaluate the presence of phenotypic markers, such as smooth muscle myosin, smooth muscle alpha-actin (Gown et al, 1985) and desmin (Ramaekers et al, 1983)(double staining with PCNA).

To estimate DNA synthesis by proliferation-associated antigens, PCNA immunohistochemistry was performed (Bravo et al, 1987; Lee and Hurwitz, 1990). For this purpose, paraffin slices $(4 \mu \mathrm{m})$ were successively incubated with mouse monoclonal anti-PCNA antibody PC 10 (overnight, $\left.4^{\circ} \mathrm{C}\right)(\mathrm{DAKO}$, 
Glostrup, Denmark), biotinylated rabbit anti-mouse secondary immunoglobulin (30 min, room temperature)(Amersham, UK), and an avidin-biotin-peroxidase conjungate (30 min, room temperature)(ABC kit, Vector Labs, Burlingame, CA, USA). Diaminobenzidine with cobalt chloride was used as chromogen. After washing, these sections were also incubated for $1 \mathrm{~h}$ with the second primary antibody desmin (Ramaekers et al, 1983) (double staining), followed by incubation ( $1 \mathrm{~h}$, room temperature) with rabbit anti- mouse peroxidase (Amersham). Sections were finally stained with diaminobenzidine. To quantify DNA synthesis, the percentage of nuclear profiles that stained for peroxidase was determined for the tunica media.

Quantitative morphology was performed on cross-sections after staining with Lawson's solution (Boom BV, Meppel, The Netherlands), which highlights the elastic laminae, using video images generated by a Zeiss Axioscope (Zeiss, Germany), a standard CCD camera (Stemmer, Germany) an commercial software (JAVA; Jandel Scientific Video Analysis Software, Jandel Scientific, Corte Madera, CA, USA). The cross-sectional area (CSA) of the tunica media was determined as the area enclosed between the internal and external elastic lamina.

\section{DNA content}

Samples of thoracic aorta and carotid artery were mechanically denuded of endothelium, weighted, frozen in liquid nitrogen and stored at $-70^{\circ} \mathrm{C}$. Their DNA content was determined as described by Labarca and Paigen (1980). Thawed tissues were solubilized in $1 \mathrm{~N} \mathrm{NaOH}(100 \mu \mathrm{l})$ for $24 \mathrm{~h}$. Of this tissue extract, $10 \mu \mathrm{l}$ was neutralized in $1 \mathrm{ml}$ phosphate buffer and added to $1 \mathrm{ml}$ of Hoechst solution (Hoechst 33258; France Biochem, Bierges, Belgium). After mixing the DNA content was measured photometrically (excitation wave length $360 \mathrm{~nm}$, emission wave length $460 \mathrm{~nm}$; AMINCO spectrophotometer, SPF500C). Calf thymus DNA (Sigma Chemicals, St Louis, MO, USA) was used as a standard.

\section{Statistics}

Differences in structural parameters between rats treated with PHE or Ang II and controls were evaluated by one-way analysis of variance followed by a t-test according to the method by Bonferroni (Wallenstein et al, 1980), using the Crunch Interactive Statistical Package (Crunch Software Corporation, San Francisco, CA, USA). P $<0.05$ was accepted to denote statistical significance of differences. 


\section{RESULTS}

\section{General observations}

Body weight, heart weight and heart rate were comparable in WKY and SHR at initiation of the study (table 8.1). Systolic blood pressure rose significantly during the 4-day study period in saline-infused SHR but not WKY (table 8.1). Infusion of $2.0 \mathrm{mg} / \mathrm{kg} /$ day PHE in WKY increased SBP to the level

Table 8.1 Effects of phenylephrine and angiotensin II on some general properties in 6week old rats".

\begin{tabular}{|c|c|c|c|}
\hline WKY & + PHE & + Ang $\mathbf{U}$ & SHR \\
\hline
\end{tabular}

\section{Before treatment}

$\begin{array}{lllll}\text { Body weight }(\mathrm{g}) & 132 \pm 5 & 130 \pm 6 & 129 \pm 7 & 128 \pm 8 \\ \text { Heart weight }(\mathrm{mg}) & 502 \pm 7 & - & - & - \\ \text { HW/BW }(\mathrm{mg} / \mathrm{g}) & 3.70 \pm 0.05 & - & - & - \\ \text { SBP (mm Hg) } & 102 \pm 5.1 & 103 \pm 6 & 109 \pm 6 & 121 \pm 8 \\ \text { HR (beats/min) } & 425 \pm 10 & 426 \pm 8 & 429 \pm 12 & 415 \pm 8\end{array}$

Following a 4-day treatment

$\begin{array}{lllll}\text { Body weight (g) } & 153 \pm 5 & 130 \pm 7 \# & 137 \pm 9 \# & 156 \pm 8 \\ \text { Heart weight (mg) } & 612 \pm 18 & 706 \pm 48 \# & 681 \pm 31 \# & 653 \pm 29 \\ \text { HW/BW (mg/g) } & 3.96 \pm 0.11 & 5.60 \pm 0.50 \# & 5.02 \pm 0.19 \# & 4.20 \pm 0.10 \\ \text { KW/BW (mg/g) } & 5.29 \pm 0.07 & 6.00 \pm 0.40 & 5.18 \pm 0.09 & 4.90 \pm 0.30 \\ \text { SBP (mm Hg) } & 107 \pm 5 & 139 \pm 7 \#, * & 184 \pm 16 \#, * & 143 \pm 3 \#, * \\ \text { HR (beats/min) } & 412 \pm 7 & 339 \pm 21 \text {, }^{*} & 342 \pm 15 \#,{ }^{*} & 433 \pm 6\end{array}$

Percent change

$\begin{array}{lllll}\text { SBP } & 6.5 \pm 5.6 & 24.3 \pm 7.4 * & 54.5 \pm 10.2 * & 24.4 \pm 5.7 * \\ \text { HR } & -3.8 \pm 2.0 & -22.8 \pm 6.0 * & 19.7 \pm 3.3 * & 5.1 \pm 1.9\end{array}$

"Findings before and after 4 days of continuous infusion are shown for WKY treated with saline (WKY), $2.0 \mathrm{mg} / \mathrm{kg} /$ day phenylephrine (+ PHE), or $0.3 \mu \mathrm{g} / \mathrm{kg} /$ day angiotensin II (+ Ang II) and for saline-infused SHR (SHR). HW/BW, heart to body weight ratio; $\mathrm{KW} / \mathrm{BW}$, left kidney to body weight ratio; SBP, systolic blood pressure; HR, heart rate. Data shown as mean \pm SEM $(n=8)$. *The change from before treatment is statistically significant $(\mathrm{p}<0.05)$. \#The difference from WKY is statistically significant $(\mathrm{p}<0.05)$. 
observed in SHR. The pressor action of $0.3 \mathrm{mg} / \mathrm{kg} /$ day Ang II was more marked. The treatments with PHE and Ang II were accompanied by a comparable tachycardia. Both vasopressors reduced body weight gain; this was more pronounced for PHE than for Ang II. Following treatment with PHE and Ang II, relative heart weight was significantly increased, while relative kidney weight was not modified.

Table 8.2 DNA synthesis in arteries of 6-week old rats'.

\begin{tabular}{lllll}
\hline Artery & WKY & + PHE & + Ang II & SHR \\
\hline aorta & $4.7 \pm 0.5$ & $8.2 \pm 1.6^{\circ}$ & $6.4 \pm 2.5$ & $9.3 \pm 1.7^{*}$ \\
carotid & $2.3 \pm 0.5$ & $3.4 \pm 1.7$ & $3.7 \pm 1.7$ & $4.7 \pm 1.5$ \\
mesenteric & $4.1 \pm 1.0$ & $5.5 \pm 0.9$ & $7.1 \pm 1.9$ & $7.7 \pm 2.0^{\circ}$ \\
renal & $6.8 \pm 1.1$ & $8.5 \pm 2.8$ & $6.2 \pm 2.4$ & $4.1 \pm 1.2$ \\
interlobar & $2.8 \pm 1.3$ & $1.4 \pm 0.4$ & $3.7 \pm 0.9$ & $1.2 \pm 0.5$ \\
femoral & $2.9 \pm 0.5$ & $6.6 \pm 1.7$ & $4.1 \pm 0.8$ & $3.4 \pm 0.8$ \\
& & & & \\
\hline
\end{tabular}

The percentage of media nuclei that incorporated BrdUrd during 4 days of infusion of the label is shown for WKY treated with saline (WKY), $2.0 \mathrm{mg} / \mathrm{kg} /$ day phenylephrine (+PHE), or $0.3 \mu \mathrm{g} / \mathrm{kg}$.day angiotensin II (+ Ang II) and for saline-treated SHR. Means \pm SEM $(n=8)$. *The difference from which WKY is statistically significant $(p<0.05)$.

\section{DNA synthesis in arteries}

Table 8.2 summarizes nuclear incorporation of BrdUrd in the tunica media of various arteries during a continuous 4-day administration of the label in young rats with and without vasopressor treatment. In saline-infused WKY, this index of DNA synthesis was most pronounced in the renal artery and least marked in the carotid artery. Differences between types of vessel were, however, not statistically significant. Compared to WKY, BrdUrd labeling was significantly elevated in the thoracic aorta and superior mesenteric artery, but not in the other vessels of SHR. PHE significantly increased DNA synthesis in the thoracic aorta. Infusions of Ang II did not increase DNA synthesis in either large and small arteries studied (table 8.2). 


\section{Cross-sectional area of arteries}

Except for the smallest arteries studied, media CSA was comparable in arteries of 6-week old WKY and SHR (table 8.3). In WKY, PHE tended to increase media CSA in several large arteries. This was statistically significant in the thoracic aorta, superior mesenteric artery and femoral artery. Ang II, on the other hand, increased media CSA in small mesenteric resistance arteries but not in the other vessels studied (table 8.3).

Table 8.3 Media cross-sectional area in arteries of 6-week old rats*.

\begin{tabular}{lllll}
\hline Artery & WKY & + PHE & + Ang II & SHR \\
\hline aorta & $330 \pm 20$ & $450 \pm 30^{\circ}$ & $380 \pm 20$ & $350 \pm 20$ \\
carotid & $100 \pm 5$ & $120 \pm 12$ & $130 \pm 10$ & $100 \pm 4$ \\
mesenteric & $110 \pm 10$ & $140 \pm 10$ & $130 \pm 10$ & $110 \pm 10$ \\
sm mesent & $2.8 \pm 0.2$ & $2.3 \pm 0.1$ & $3.9 \pm 0.6^{\circ}$ & $3.8 \pm 0.2^{\circ}$ \\
renal & $2.8 \pm 1.3$ & $1.4 \pm 0.4$ & $3.7 \pm 0.9$ & $1.2 \pm 0.5$ \\
femoral & $70 \pm 6$ & $92 \pm 12$ & $75 \pm 6$ & $73 \pm 6$ \\
& & & & \\
\hline
\end{tabular}

"Media cross-sectional area $(\mu \mathrm{mx} 1000)$ is shown for 6-week old WKY treated during 4 days with saline (WKY), $2.0 \mathrm{mg} / \mathrm{kg} /$ day phenylephrine (+PHE), or $0.3 \mu \mathrm{g} / \mathrm{kg}$.day angiotensin II ( + Ang II) and for saline-treated SHR. Means \pm SEM $(n=8)$.

${ }^{b}$ Mesenteric small artery, i.e., 4- to 5-order side-branch of the superior mesenteric artery. *The difference from which WKY is statistically significant $(p<0.05)$.

\section{DNA content in thoracic aorta and carotid artery}

In both the thoracic aorta and carotid artery, neither the development of hypertension in SHR nor the treatment of WKY with PHE or Ang II had any effect on DNA content per unit of weight $(\mu \mathrm{g} / \mathrm{mg})$ or DNA content per unit of lenght $(\mu \mathrm{g} / \mathrm{mm})$ (table 8.4$)$.

\section{Proliferating cell nuclear antigen and desmin in thoracic aorta}

In the thoracic aorta, nuclear staining for the endogenous PCNA cyclin, was more marked than nuclear incorporation of BrdUrd (table 8.1 and 8.5). It was significantly increased by Ang II, but did not differ between the other treatment groups (table 8.5). 
Table 8.4 DNA content of the thoracic aorta and carotid artery of 6 week old rats ${ }^{a}$.

\begin{tabular}{|c|c|c|c|c|}
\hline Aorta & WKY & PHE & Ang II & SHR \\
\hline $\begin{array}{l}\text { Tissue length } \\
(\mathrm{mm})\end{array}$ & $24.8 \pm 1.0$ & $25.3 \pm 0.6$ & $25.9 \pm 1.0$ & $24.9 \pm 0.4$ \\
\hline $\begin{array}{l}\text { Tissue wet } \\
\text { weight (mg) }\end{array}$ & $17.5 \pm 1.2$ & $20.2 \pm 1.1$ & $20.8 \pm 2.1$ & $16.7 \pm 0.7$ \\
\hline Total DNA $(\mu \mathrm{g})$ & $57.3 \pm 5.0$ & $62.0 \pm 2.8$ & $63.4 \pm 6.3$ & 58.11 .6 \\
\hline DNA $(\mu \mathrm{g} / \mathrm{mg})$ & $3.3 \pm 0.1$ & $3.1 \pm 0.2$ & $3.1 \pm 0.1$ & $3.5 \pm 0.2$ \\
\hline DNA $(\mu \mathrm{g} / \mathrm{mm})$ & $2.3 \pm 0.1$ & $2.4 \pm 0.2$ & $2.4 \pm 0.1$ & $2.3 \pm 0.6$ \\
\hline Carotid artery & WKY & PHE & Ang II & SHR \\
\hline $\begin{array}{l}\text { Tissue length } \\
(\mathrm{mm})\end{array}$ & $12.5 \pm 0.3$ & $12.0 \pm 0.6$ & $12.1 \pm 0.4$ & $11.8 \pm 0.7$ \\
\hline $\begin{array}{l}\text { Tissue wet } \\
\text { weight }(\mathrm{mg})\end{array}$ & $3.5 \pm 0.3$ & $3.2 \pm 0.2$ & $3.5 \pm 0.2$ & $2.9 \pm 0.2$ \\
\hline Total DNA $(\mu \mathrm{g})$ & $8.2 \pm 0.1$ & $8.1 \pm 0.4$ & $8.4 \pm 0.2$ & $8.2 \pm 0.6$ \\
\hline DNA $(\mu \mathrm{g} / \mathrm{mg})$ & $2.4 \pm 0.2$ & $2.6 \pm 0.1$ & $2.5 \pm 0.1$ & $2.9 \pm 0.1$ \\
\hline DNA $(\mu \mathrm{g} / \mathrm{mm})$ & $0.69 \pm 0.01$ & $0.67 \pm 0.01$ & $0.70 \pm 0.03$ & $0.70 \pm 0.01$ \\
\hline
\end{tabular}

'Length, wet weight, total DNA content, DNA content per unit of weight and DNA content per unit of length are shown for thoracic aorta and carotid artery of 6 week old WKY infused during 4 days with saline (WKY), $2.0 \mathrm{mg} / \mathrm{kg} /$ day PHE or $0.3 \mathrm{mg} / \mathrm{kg} /$ day Ang II and for 6 week old SHR. Data are shown as means \pm SEM $(n=7-8)$.

Desmin was present in approximately $40 \%$ of the media cells of the thoracic aorta of 6-week old WKY. The presence of desmin containing cells was comparable in SHR and WKY (table 8.5). It was significantly increased in WKY treated with PHE but not in WKY that had been infused with Ang II (table 8.5). PCNA staining was more pronounced in cells that contained desmin than in those in which desmin could not be demonstrated (table 8.5). This was the case for all 4 treatment groups. For all animals combined $(n=29)$, overall PCNA staining averaged $15 \pm 2 \%$, PCNA labeling in media cells without desmin $9 \pm 1 \%$, and that in cells with desmin $25 \pm 3 \%$. Table 8.5 also indicates that Ang II stimulated the presence of PCNA primarily in the nuclei of media 
cells that contained desmin.

The distribution of desmin, actin and myosin (table 8.6) suggests that the media of the thoracic aorta of 6-week old rats is composed of a mixture of smooth muscle cells with different degree of differentiation. In the thoracic aorta of 20 week old rats, their is almost a doubling of cells that stain for desmin (table 8.6).

Table 8.5 PCNA and desmin in the thoracic aorta media of 6-week old rats".

\begin{tabular}{lllll}
\hline & WKY & + PHE & + Ang II & SHR \\
\hline & & & & \\
PCNA & $11 \pm 2$ & $11 \pm 2$ & $19 \pm 3^{\circ}$ & $17 \pm 6$ \\
Desmin & $34 \pm 1$ & $41 \pm 3^{\circ}$ & $38 \pm 3$ & $33 \pm 4$ \\
PCNA in D- & $7 \pm 1$ & $5 \pm 1$ & $10 \pm 3$ & $11 \pm 3$ \\
PCNA in D+ & $20 \pm 4$ & $19 \pm 3$ & $34 \pm 5^{\circ}$ & $27 \pm 8$ \\
& & & & \\
\hline
\end{tabular}

The percentage of nuclei containing PC.NA and the percentage of cells containing desmin in their cytoplasm is shown. PCNA in D- and PCNA in D+ refer to the percentage of nuclei staining for PCNA in cells lacking and containing desmin, respectively. These observations are show for 6-week old WKY infused during 4 days with saline (WKY), 2.0 $\mathrm{mg} / \mathrm{kg} /$ day phenylephrine (+PHE), or $0.3 \mu \mathrm{g} / \mathrm{kg}$.day angiotensin II ( + Ang II) and for saline-treated SHR. Means \pm SEM $(n=7-8)$.

The difference from which WKY is statistically significant $(p<0.05)$.

\section{DISCUSSION}

The findings of the present study indicate that structural responses to elevated blood pressure, angiotensin II and phenylephrine are heterogeneously distributed over the arterial system of young rats. Beside differences between types of blood vessel, also differences were observed between subtypes of smooth muscle cells within a given arterial segment.

In essential hypertension and in spontaneously hypertensive rats, vascular resistance is increased as a result of arterial structural changes (Folkow, 1982). These structural changes differ between arteries of different anatomical origin, i.e. large (ASMC hypertrophy/hyperploidy) and small (ASMC hyperplasia) arteries (Schwartz et al, 1986; Owens, 1989; Mulvany et al, 1985; Owens et al, $\left.1988^{a}\right)$. The reasons for this regional heterogeneity are not clear. Beside phenomena such as regional differences in hemodynamics and mechanisms 
Table 8.6 Phenotype markers in rat aorta media'.

\begin{tabular}{lll} 
Age & 6 weeks & 20 weeks \\
\hline & & \\
SM myosin & $>99 \%$ & $>99 \%$ \\
SM alpha-actin & $84 \pm 3 \%$ & $>99 \%$ \\
Desmin & $37 \pm 3 \%$ & $67 \pm 2 \%$ \\
Non-muscle myosin & $11 \pm 4 \%$ & $3 \pm 1 \%$ \\
\hline
\end{tabular}

"Percent of thoracic aorta media cells staining for smooth muscle myosin (SM myosin), smooth muscle alpha-actin (SM alpha-actin), desmin and non-muscle myosin, are shown for 6 and 20 week old rats. Data are shown as means \pm SEM $(n=8-32)$.

endogenous to the vascular wall, also a non-uniform input of mitogenic stimuli could play a role (Owens, 1988; Bevan and Tsuru, 1981; Schwartz et al, 1986; Davies, 1986; Thyberg et al, 1990). Several lines of evidence indicate that both the sympathetic nervous system (SNS) and the renin-angiotensin system (RAS) play a trophic role during pathological and experimental structural changes in the vasculature (Head, 1991; Lee and Smeda, 1985; Sahlgren et al, 1986; Lever, 1986). In addition, these neurohumoral mechanisms most likely interact, whereby Ang II can promote sympathetic nerve activity both centrally and peripherally (Severs and Severs-Daniels, 1973; Zimmerman, 1978; Vanhoutte et al, 1981). Because the density of periarterial nerves varies considerably along the vascular tree (Abboud, 1984), we investigated whether infusion of pressor doses of Ang II or PHE altered the structure of various arteries differently.

In WKY treated with PHE, an increase in blood pressure was observed comparable to that in SHR. Ang II increased blood pressure even higher than that observed in SHR. Yet, PHE, but not Ang II, increased DNA synthesis and media cross-sectional area in the thoracic aorta. Pressor treatment did also not significantly alter the aortic side-branches that were investigated while we described that Ang II, but not PHE, increased media cross-sectional area in mesenteric resistance arteries of the same animals. Like mesenteric resistance of WKY treated with Ang II, vessels of SHR showed an increased media CSA. These observations suggest that a major role for pressure-mediated effects in agonist-stimulated growth response of arteries seems to be unlikely in young rats. In addition, these results may also explain the more marked increase in blood pressure in Ang II treated WKY, that could be the result of the 
contractile effect of Ang II in combination with a structural change of peripheral resistance-sized arteries.

A role for sympathetic nerves in the regionality of PHE and Ang II action also seems unlikely. Periarterial sympathetic nerves are virtually absent in the rat thoracic aorta but very dense in femoral arteries and mesenteric resistance arteries (Abboud, 1984). In the former, PHE, but not Ang II, increased DNA synthesis and media cross-sectional area, which could be compatible with a nervous contribution to Ang II action. Yet, in neither femoral artery nor mesenteric resistance artery, a trophic action of PHE was detected and Ang II resulted in media hypertrophy in the small vessel, but not in the large artery. This does not exclude that Ang II could act through a nonadrenergic neurotransmitter but is incompatible with the sensitivity of the mitogenic action of Ang II to prazosin in large arteries of adult rats (van Kleef et al, 1992).

That alpha-1 stimulation and Ang II can promote SMC growth through direct effects on the SMC's is supported by observations in cell culture (Blaes and Boissel, 1983; Nakaki et al, 1990; Yamori et al, 1987; Daemen et al, 1991; Berk et al, 1989; Geisterfer et al, 1988). Also these in-vitro findings, however, are not uniform. Effects of norepinephrine and epinephrine on DNA synthesis have been attributed to alpha- 1 and beta-adrenoceptor stimulation in different studies. Mitogenic effects of Ang II may be counteracted by production and activation of TGF- $\beta$ (Gibbons et al, 1992). The intensity of this autocrine feedback varies, leading to SMC proliferation in one experimental setting and to SMC hypertrophy in another. Similar phenomena could be responsible for regional heterogeneity of the effect of PHE and Ang II on DNA synthesis and media cross-sectional area observed in the present study and for the differences between this study in young rats and earlier observations in adult animals. Differences in receptor subtype distribution may also b e worth considering. At least 3 subtypes of alpha-1 adrenoceptors have now been cloned (Flavahan and Vanhoutte, 1986; Han et al, 1990; Ruffolo et al, 1991). They differ most notably in the signal transduction pathway which they activate raising the possibility that they participate to a different extent in growth control. It was previously recognized that the pharmacological properties of alpha-1 vasoconstrictor responses vary considerably along the arterial tree (De Mey and Vanhoutte, $1981^{\text {a) }}$. This does not fully correspond to the abovementioned receptor subtypes further stressing the diversity of vascular alpha-1 adrenergic mechanisms. Also in the case of Ang II multiple receptor subtypes have been identified (Viswanathan et al, 1991; Chiu et al, 1991). The expression of these subtypes changes during development (Viswanathan et al, 1991). In rat aorta, AT-2 receptors predominate in the neonate, while AT-1 receptors prevail in the adult. The situation in other vessels remains to be established. Yet, because the majority of actions of Ang II seem to be mediated 
by AT-1 receptors, temporal and regional differences in receptor subtype availability could participate in the heterogeneity of the trophic actions of Ang II in time and in space.

So far, we concentrated on differences between different branches of the arterial tree. Also within a given segment, however, arterial smooth muscle cells may not be uniform. This intravascular heterogeneity may be one of the mechanisms contributing to intervessel heterogeneity. Our observations on desmin in the aorta of 6-week old rats confirm earlier observations that especially in young rats the aortic media is composed of a mixture of ASMC with different degrees of differentiation. This conclusion is based on presence of selected cytoskeletal and contractile proteins. These were previously used together with ultrastructural criteria in support of the so-called phenotype modulation hypothesis (Chamley-Campbell et al, 1979, 1981; Schwartz et al, 1986; Thyberg et al, 1990). This stated that ASMC had to change from a contractile type into a synthetic phenotype prior to proliferation. In recent years, a number of in-vitro findings indicated, however that ASMC phenotype changes were not causally related to ASMC proliferation. Also in the present study, we found that DNA polymerase $\delta$, which is essential for DNA synthesis, was most frequent in aortic cells containing desmin. This is in contrast to the phenotype modulation hypothesis. As an alternative, it has been proposed on the basis of findings in human atherosclerotic plaques, experimental neointimas and cell culture experiments, that SMC proliferation would be limited to a subset ASMC's (Schwartz et al, 1986; Chamley-Campbell, 1979, 1981; Blank et al, 1988; Skalli et al, 1986). Regional heterogeneity of the distribution of these along the vascular tree may be responsible for differences between types of vessel. Its corroboration awaits the use of workable markers for eventual SMC subtypes in a comparative study. It may be worth to include alpha- and AT-receptor subtypes in this analysis.

Because in this study PHE of Ang II were infused during only 4 days, the structural consequences of the observed regionality and agonist selectivity may not be fully inferred from the present findings. Although the treatment was sufficiently long to result in cardiac hypertrophy with both agents, aortic hypertrophy with PHE and resistance artery hypertrophy with Ang II, it cannot be excluded that other combinations of agent and vessel would reach statistical significance during longer treatment. Furthermore, increased DNA synthesis stimulated by PHE in the aorta was not accompanied by increased DNA content. In chapter 7 , it was shown that elevated DNA synthesis in large arteries of 6 week old SHR did not result in increased DNA content at 20 weeks of age. ASMC turnover may previously have been underestimated. In addition, Ang II stimulated the presence of PCNA but not DNA synthesis. This type of findings along with the above-mentioned suggestion of ASMC subtypes leads us to the following proposition. Growth responses may be limited to a 
subpopulation of ASMC. The size of this population and its growth rate may vary locally and may be influenced by agonists. Ang. II and alpha-1 adrenoceptor stimulation may operate in series: entry and progression through the cell cycle and initiation of S-phase possibly were affected to a different extent by both vasoconstrictors. Differences in this respect between arteries of young and adult rats and between the media and experimental neointima in adult rats may find their origin in a different degree of ASMC differentiation. In addition to the above-mentioned comparative studies of subtype markers, corroboration of this proposition will depend on the outcome of experiments in which cell cycle markers are evaluated following stimulation with a combination of Ang II and alpha-1 agonist.

In summary, administration of a pressor dose of PHE was found to stimulate DNA synthesis and media hypertrophy in the thoracic aorta, but not other arteries of young rats. Raising blood pressure with Ang II did, however, not modify DNA synthesis or cross-sectional area in either of the large arteries studied. That Ang II stimulated the presence of PCNA in the aorta of young rats, but not DNA synthesis, may help to unravel the interaction between Ang II and alpha-1 adrenoceptors in the control of experimental arterial growth responses. 


\section{Chapter}

\section{Tonic Tone in Arteries Exposed Continuously to Angiotensin II in Vitro}

P.M.H. Schiffers, H.A.M.D. van der Heijden, G.E. Fazzi, H.A.J. Struijker-Boudier and J.G.R. De Mey

Department of Pharmacology, University of Limburg, Maastricht, the Netherlands. (Journal of Pharmacology and Experimental Therapeutics 1993, 266: 1520-1527) 


\section{INTRODUCTION}

Angiotensin II (Ang II) plays a pivotal role in cardiovascular homeostasis and electrolyte balance (Dzau, 1988). Ang II elicits various acute effects that may mediate its acute pressor action, such as vasoconstriction, facilitation of adrenergic neurotransmission and stimulation of aldosterone production (Vanhoutte et al, 1981; Dzau, 1988). Continuous infusion of low doses of Ang II in intact animals, on the other hand, results in a slowly developing progressive increase in blood pressure (Brown et al, 1981; Griffin et al, 1991). The nature of this "slow pressor action", that may underlay the role of Ang II in hypertension (Lever, 1986), largely remains to be established. Candidate mechanisms include (i) a trophic action of the peptide on arterial smooth muscle (Berk et al, 1989 $9^{\text {; }}$ Owens, 1989; Griffin et al, 1991; Turla et al, 1991), (ii) stimulation of the production of vasoconstrictors within the arterial wall (Dzau, 1988; Dohi et al, 1992), (iii) potentiation of sympathetic nerve activity (Phillips, 1987; Lappe and Brody, 1984), and (iv) elevation of intracellular $\mathrm{pH}$ and sodium concentration in arterial smooth muscle (Kuriyama et al, 1988; Vallega et al, 1988).

The goal of the present study was to evaluate whether, in analogy to the acute and slow pressor action in vivo, Ang II elicits besides a fast transient contraction also a slow chronic elevation of tone in isolated arteries. Furthermore the structural or functional nature of this long term vascular action was evaluated. For this purpose we recorded isometric force development and structural parameters in isolated arterial segments before, during and following culture in the absence or presence of Ang II. The experiments were performed in rat renal artery because this preparation (i) was previously observed to display a transient acute contractile effect to Ang II (De Mey et al, 1989), (ii) can develop in vitro mitogenic responses that are considerably stronger than those in other blood vessels (De Mey et al, 1991c), and (iii) may share properties with other parts of the renal vascular bed which plays a pivotal role in hypertension.

\section{MATERIALS AND METHODS}

Renal arteries were isolated under sterile conditions from 20 week old male Wistar-Kyoto rats (local inbred). All vessels were chemically sympathectomized with 6-hydroxydopamine (Aprigliano and Hermsmeyer, 1976) and were, except when specifically mentioned, mechanically denuded of endothelium (De Mey et al, 1991c).

To record isometric force development during a three day period, arterial segments $(2 \mathrm{~mm}$ long) were mounted horizontally in a sterile stainless steel 
organ chamber between an isometric force transducer (Grass UC3, Hugo Sachs, FRG) and a displacement device. The organ chamber was filled with 10 $\mathrm{ml}$ culture medium that was maintained at $37^{\circ} \mathrm{C}$ and overflown with moist $95 \%$ $\mathrm{O}_{2}-5 \% \mathrm{CO}_{2}$. The culture medium consisted of Dulbecco's Eagle's modified medium (DMEM; Gibco, Paisley, UK), containing $2 \mathrm{mM}$ glutamine (Gibco), $100 \mu \mathrm{g} / \mathrm{ml}$ streptomycin (Pharmachemie, Haarlem, The Netherlands), 100 $\mathrm{IU} / \mathrm{ml}$ penicillin (Gist-brocades, Delft, The Netherlands) and $5 \%$ dialyzed human plasma-derived serum stripped from progression factors (CMS-PDS, Vogel et al, 1978). The arterial preparations were stretched to an internal circumference of $2.8 \mathrm{~mm}$ previously found to represent optimal distension for isometric force development (De Mey et al, 1989). The preparations were then allowed to stabilize during $60 \mathrm{~min}$ after which part of the arterial segments were exposed to $1 \mu \mathrm{M}$ Ang II. In four experiments, samples of the incubate were withdrawn at 1 hour interval and their Ang II content was determined by radioimmunoassay. This indicated that no significant degradation of the peptide occurred over a 6 hour period. Culture medium with or without Ang II was replaced every 6 hours during 3 days and wall tension was recorded continuously. Part of these experiments were performed in the presence of $1 \mu \mathrm{g} / \mathrm{ml}$ indomethacin. 96 Hours after the start of tension recording, effects of the nonselective angiotensin receptor antagonist saralasin (Sar) (Dudley et al, 1990), the endothelin ETa-receptor antagonist BQ123 (Ihara et al, 1992) and the vasodilator sodium-nitroprusside (SNP) were evaluated. Subsequently the organ chamber content was replaced several times by drug-free Krebs-Ringer bicarbonate solution (KRB) that had been preheated $\left(37^{\circ} \mathrm{C}\right)$ and aerated. The preparations were allowed to adapt to these conditions for $90 \mathrm{~min}$ and contractile responses to high potassium (K-KRB), serotonin (5HT), and Ang II were recorded (fig. 9.1). Furthermore, effects of potassium removal and readministration and effects of $10 \mathrm{mM} \mathrm{NH} \mathrm{NH}_{4} \mathrm{Cl}$ and its removal were evaluated during contractions induced by $2 \mu \mathrm{M} 5 \mathrm{HT}$. These protocols have previously been used to evaluate effects on tone of electrogenic sodium transport, cellular $\mathrm{pH}$ and activation of sodium-hydrogen exchange (De Mey and Vanhoutte, $1982^{\text {b; }}$ Hermsmeyer and Harder, 1986; Madshus, 1988; Aalkjaer, 1990; Kahn et al, 1990). For comparison, effects of contractile agents and ionic interventions were also evaluated in arterial segments that had not been maintained in culture. Freshly isolated preparations were therefore mounted in organ chambers containing KRB, stretched to the appropriate circumference and exposed to contractile agents immediately after sympathectomy and removal of endothelium.

In a second series of experiments, arterial segments $(6 \mathrm{~mm}$ long) were maintained in vitro during 3 days at an internal circumference of $2.8 \mathrm{~mm}$ in DMEM plus CMS-PDS without continuous recording of wall tension. The preparations were incubated in $2.5 \mathrm{ml}$ medium containing $1 \mu \mathrm{g} / \mathrm{ml}$ 
indomethacin with or without Ang II. The medium was replaced and harvested $\left(-20^{\circ} \mathrm{C}\right)$ at 24 hour interval. After 3 days of culture the arterial preparations were recovered and divided in a segment that was used for structural evaluation and a segment used for additional contractile measurements. The latter consisted of the test protocol outlined above (fig. 9.1) and a bioassay aimed at evaluating the presence of a contractile factor in media conditioned by arteries in the continuous absence or presence of Ang II. For this purpose, harvested media $(2.5 \mathrm{ml})$ were mixed with $7.5 \mathrm{ml} \mathrm{KRB}$ and were added to the arterial segments after they had been desensitized for the acute contractile effect of the peptide through repeated exposure.

For structural evaluation, preparations were fixed in formalin, embedded in paraffin and cross sectioned $(4 \mu \mathrm{m})$. Following staining with Lawson's solution, media cross sectional area was determined. The area enclosed by the internal and external elastic lamina was measured using images generated by a Zeiss Axioscope equipped with a standard CCD camera (Stemmer, Germany) and commercially available software (JAVA 1.21, Jandel Scientific, Corte Madera, CA, USA).

In a third series of experiments, culture with and without Ang II was performed in the continuous presence of $1 \mu \mathrm{M}$ of the thymidine-analogue, BrdUrd, to estimate DNA synthesis. Nuclear incorporation of BrdUrd was visualized by a monoclonal antibody (Eurodiagnostics, Appeldoorn, The Netherlands) and peroxidase-conjugated second step reagent on cross sections counterstained with haematoxylin and eosine (Gratzner, 1982; De Mey et al, $\left.1989,1991^{\mathrm{C}}\right)$. The percentage of labeled cells was determined.

$\mathrm{KRB}$ had the following composition (in $\mathrm{mM}$ ): $\mathrm{NaCl} 118.3, \mathrm{KCl} 4.7$, $\mathrm{CaCl}_{2}$ 2.5, $\mathrm{MgSO}_{4} \cdot \mathrm{H}_{2} \mathrm{O} 1.2, \mathrm{KH}_{2} \mathrm{PO}_{4} 1.2, \mathrm{NaHCO}_{3}$ 25.0, glucose 11.1. For $\mathrm{K}-\mathrm{KRB}$ all $\mathrm{NaCl}$ was replaced by an equimolar amount of $\mathrm{KCl}$. In potassiumfree $\mathrm{KRB}, \mathrm{KCl}$ was omitted and $\mathrm{KH}_{2} \mathrm{PO}_{4}$ was replaced by $\mathrm{NaH}_{2} \mathrm{PO}_{4}$. Rat Ang II, 6-hydroxydopamine, indomethacin, and Sar were obtained from Sigma Chemicals (Saint Louis, Mo, USA). 5HT creatinine sulphate and SNP were from Janssen Chimica (Beerse, Belgium) and rat endothelin-1 was purchased from Peptides Int. (Louisville, KT, USA). BQ123 (cyclo-[D-Asp-L-Pro-D-ValL-Leu-D-Trp]) was a gift from E. Ohlstein (SKF, King of Prussia, PA, USA).

All observations are shown as mean \pm SEM, with $n$ indicating the number of preparations. Statistical significance of differences was evaluated by analysis of variance (Wallenstein et al, 1980). 


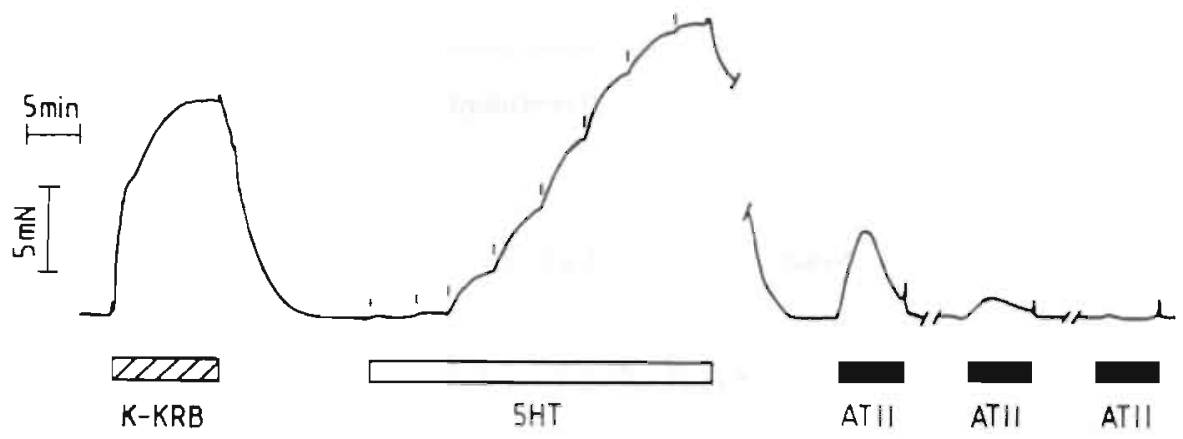

Figure 9.1 Tracing of isometric force versus time illustrating contractile responses to $\mathrm{K}$ $\mathrm{KRB}(\oslash), 0.01$ to $30 \mu \mathrm{M}$ 5HT ( $\square$ ) and $1 \mu \mathrm{M}$ Ang II ( $\square$ ) in a freshly isolated sympathectomized renal segment that had been denuded of endothelium. At the breaks the recording was stopped during $30 \mathrm{~min}$.

\section{RESULTS}

\section{Tonic arterial tone during continuous exposure to Ang II}

In freshly isolated sympathectomized renal artery segments that had been denuded of endothelium and incubated in $\mathrm{KRB}, \mathrm{K}-\mathrm{KRB}$, and $5 \mathrm{HT}$ induced strong contractions that were well maintained (fig. 9.1). $1 \mu \mathrm{M}$ Ang II induced an acute increase in force that was relatively small and highly transient (fig. 9.1). As noted before, repeated administration of Ang II yielded progressively smaller contractile responses (fig. 9.1, table 9.1).

Figure 9.2 illustrates isometric force in vessel segments exposed for three days to culture medium with and without $1 \mu \mathrm{M}$ Ang II. During this period, wall tension changed minimally in the absence of the peptide. In the vessels continuously exposed to Ang II, the tension response can be subdivided in three phases. Initially an acute increase in force was observed. Though being significantly larger than the one observed in physiological salt solution, also this response faded rapidly despite continuous presence of Ang II. From 30 min to 12 hours, tension did not differ from that in vessels not exposed to the peptide. Thereafter, force started to increase slowly and reached a stable level after approximately 48 hours (40 to $70 \%$ of the contractile response to K$\mathrm{KRB}$ ). This tonic contraction was then maintained for at least 24 more hours (fig. 9.2). It was not affected by addition of $1 \mu \mathrm{M}$ Sar (fig. 9.3), but could be 


\section{CHAPTER 9}

Table 9.1 Effects of vasoconstrictors and conditioned media in arterial segments ${ }^{\mathrm{a}}$

\section{Treatment of Arteries}

Stimulus

None Culture Culture+ Ang I

$\mathrm{K}-\mathrm{KRB}(\mathrm{mN} / \mathrm{mm})$

$2.9 \pm 0.3(12)$

$2.7 \pm 0.4(12)$

$3.0 \pm 0.4(12)$

$10 \mu \mathrm{M}$ 5HT (\%)

$148 \pm 8(12)$

$145 \pm 9(12)$

$122 \pm 8(12)$

1st $1 \mu \mathrm{M}$ Ang II (\%)

$47 \pm 4 \quad(6)$

$36 \pm 5 \quad(6)$

$35 \pm 7 \quad(6)$

2nd $1 \mu \mathrm{M}$ Ang II (\%) $11 \pm 7$ (6)

$8 \pm 4$ (6)

$9 \pm 5 \quad(6)$

3rd $1 \mu \mathrm{M}$ Ang II (\%)

$3 \pm 2(6)$

$1 \pm 3(6)$

$0 \pm 3(6)$

Conditioned media

$\begin{array}{llll}\text { control (\%) } & \text { ND } & 4 \pm 3(6) & 3 \pm 5 \text { (6) } \\ \text { with Ang II (\%) } & \text { ND } & 4 \pm 3(6) & 6 \pm 4(6)\end{array}$

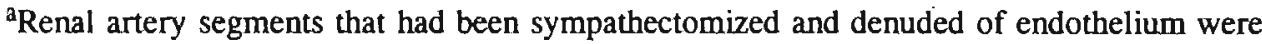
mounted for recording of force development at an internal circumference of $2.8 \mathrm{~mm}$. Experiments were performed immediately after isolation, after three days of culture in indomethacin-containing medium, or after culture in the presence of both indomethacin and $1 \mu \mathrm{M}$ Ang II. Acute effects of Ang II were tested three times at 30 min interval to evaluate tachyphylaxis. Contractile effects of conditioned media were evaluated following this desensitization. Media were used that had been exposed to arterial segments for 24 hours in the absence or presence of Ang II. Findings are shown that were obtained with media harvested following the third culture day (observations with media of day 1 and day 2 , were identical).

Data were expressed as increase in wall tension $(\mathrm{mN} / \mathrm{mm})$ or as percentage of the contractile response to $\mathrm{K}-\mathrm{KRB}$ and are shown as mean $\pm \mathrm{SEM}(\mathrm{n}=6-12)$. ND, not determined. 

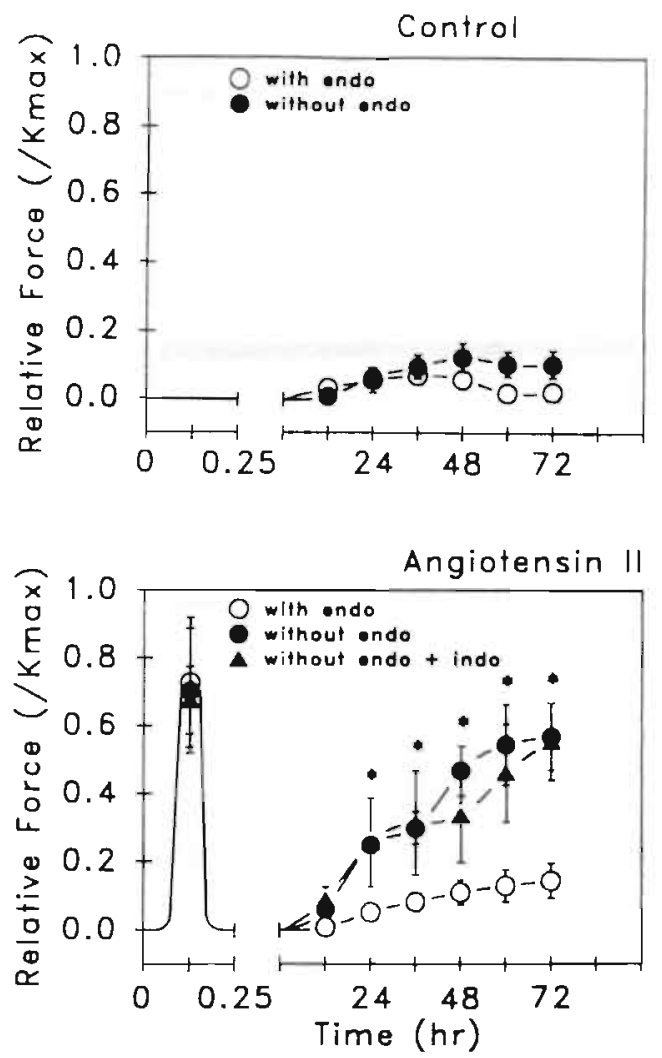

Figure 9.2 Results of continuous recording of active wall tension during organ culture of sympathectomized renal artery segments. The experiments were performed in continuous absence (top) or presence (bottom) of $1 \mu \mathrm{M}$ Ang II which was added at time 0 . Both vessels with endothelium $(O)$ and segments without endothelium $(\bullet)$ were used. Part of the study was performed in the presence of $1 \mu \mathrm{g} / \mathrm{ml}$ indomethacin $(\boldsymbol{\Lambda})$. The results were expressed as fraction of the contractile response to K-KRB determined $90 \mathrm{~min}$ after removal of the culture medium (see table 1) and are shown as mean \pm SEM $(n=6-12)$. The long term effect of Ang $\Pi$ is statistically significant in denuded vessels but not in vessels with endothelium. Differences between absence and presence of endothelium are not significant.

reversed by $1 \mu \mathrm{M}$ SNP (fig. 9.3). Also replacing the angiotensin-containing medium by drug-free physiological salt solution, abolished the tonic force (fig. 9.3).

In renal artery segments had not been denuded of endothelium, the acute 


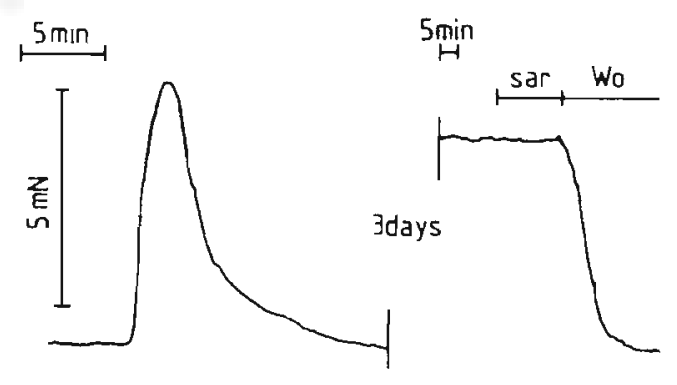

ATII

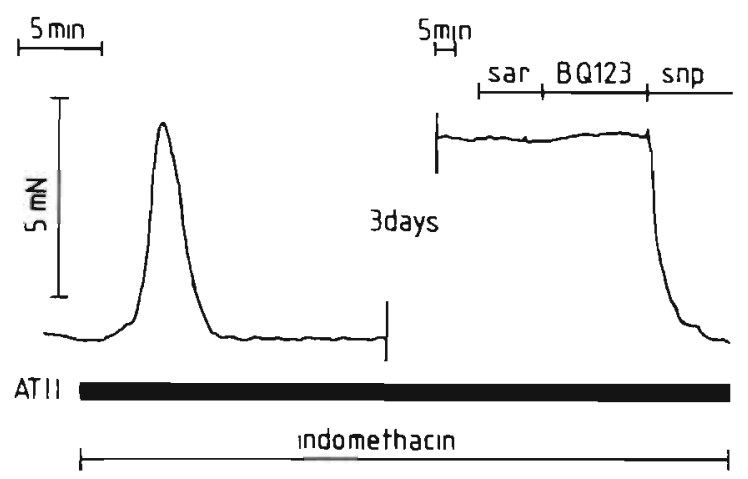

Figure 9.3 Typical tracings of isometric force versus time illustrating effects of $1 \mu \mathrm{M}$ sar, 1-10 $\mu \mathrm{M}$ BQ123, $1 \mu \mathrm{M}$ SNP (snp) and agonist-removal (Wo) on tonic tone in arteries exposed for three days to $1 \mu \mathrm{M}$ Ang II ( $\boldsymbol{\square})$. The experiments were performed in continuous absence (top) or presence (bottom) of $1 \mu \mathrm{g} / \mathrm{ml}$ indomethacin which was added at time 0 . Similar findings were obtained in at least five more experiments.

transient contractile effect of the peptide was comparable to that in denuded vessels (fig. 9.2). The long term tonic contractile effect of Ang II was, however, markedly smaller in vessels with endothelium than in preparations without endothelium (fig. 9.2).

\section{Candidate mechanisms}

\section{Autocrine vasoconstrictor production}

Long term exposure of isolated renal artery segments to Ang II with continuous recording of wall tension was repeated in the continuous 


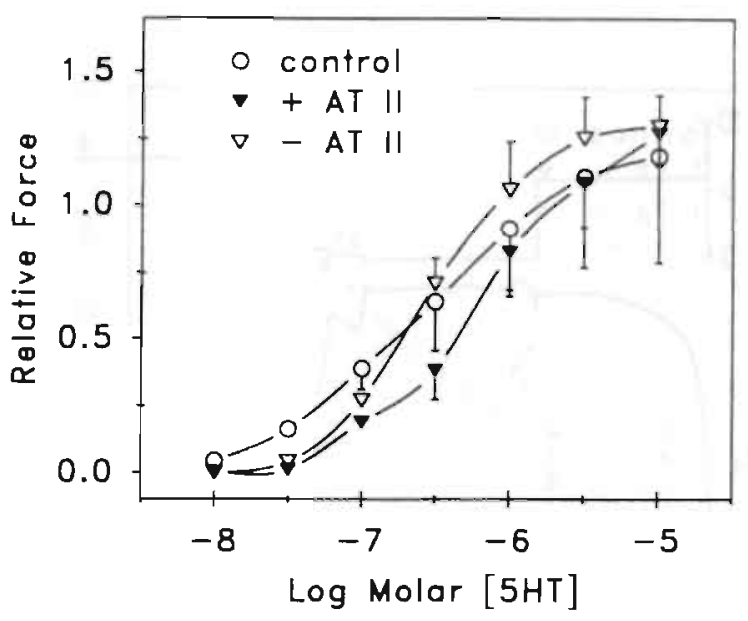

Figure 9.4 Concentration response curves for the contractile effect of 5HT in freshly isolated renal artery segments $(O)$ and preparations maintained in culture for 3 days in the absence $(\nabla)$ or presence $(\boldsymbol{\nabla})$ of $1 \mu \mathrm{M}$ Ang II. Data were expressed as fraction of the contractile response to K-KRB (see table 1 ) and are shown as means \pm SEM $(n=6)$.

presence of $1 \mu \mathrm{g} / \mathrm{ml}$ indomethacin (fig. 9.2). Observations did not differ from those in absence of cyclooxygenase inhibitor. The amplitude and time course of both the acute transient and long term tonic response to Ang II were not modified by blockade of prostaglandin production (fig. 9.2). Furthermore, the tonic tone in these vessels was not modified by 1 to $10 \mu \mathrm{M}$ BQ123 (fig. 9.3). In preliminary experiments $(n=4)$ this agent reversed contractions induced by $30 \mathrm{nM}$ endothelin-1 in freshly isolated arteries.

In additional experiments, indomethacin-containing media that had been conditioned by renal arteries were evaluated for contractile activity. These bioassay experiments were performed with assay rings prepared from vessels that had or had not been exposed to Ang II for three days (table 1) and that had been desensitized for the acute contractile effect of the peptide. Both media conditioned in the presence of Ang II and media without addition of Ang II were used. In none of the donor-sensor combinations used, the conditioned media elicited a significant contractile effect (table 9.1). 
A
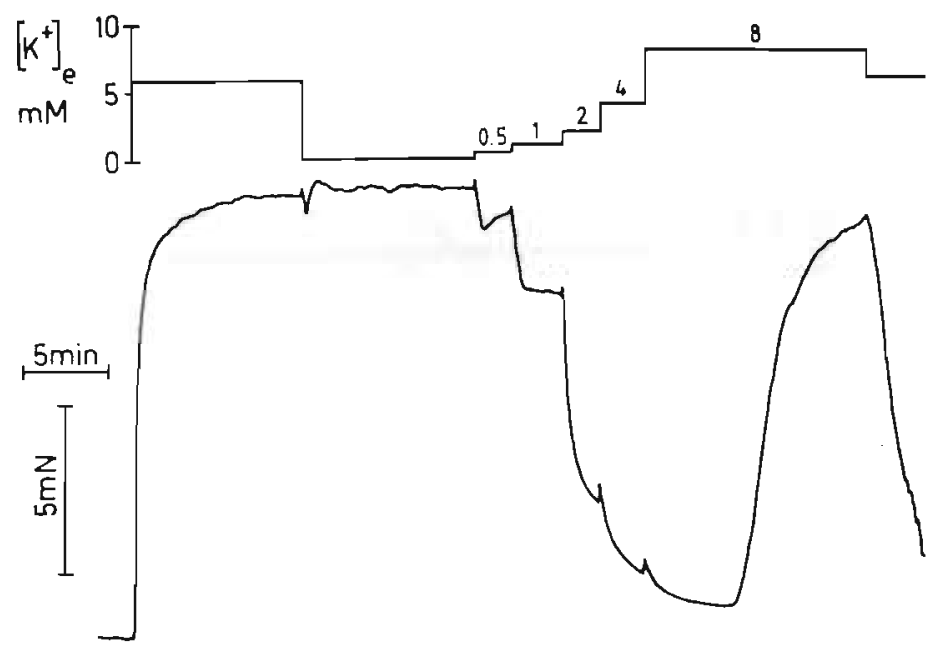

B

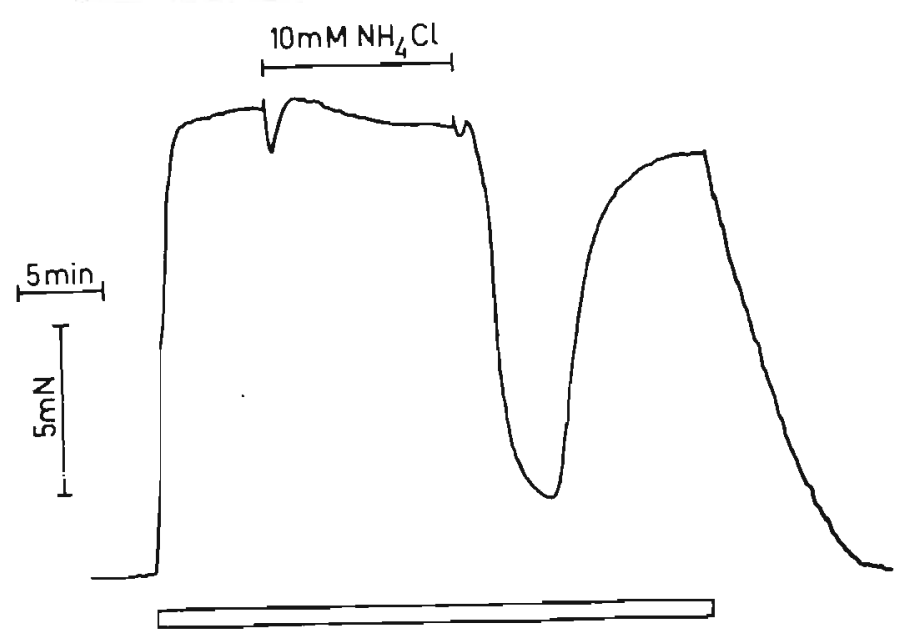

Figure 9.5 Tracings of isometric force versus time illustrating effects of A: changes in extracellular potassium concentration $([\mathrm{K}+] \mathrm{e})$ and $\mathrm{B}: 10 \mathrm{mM} \mathrm{NH} \mathrm{NH}_{4} \mathrm{Cl}$ on contractile responses to $2 \mu \mathrm{M} 5 \mathrm{HT}(\square)$ in rat renal artery. 


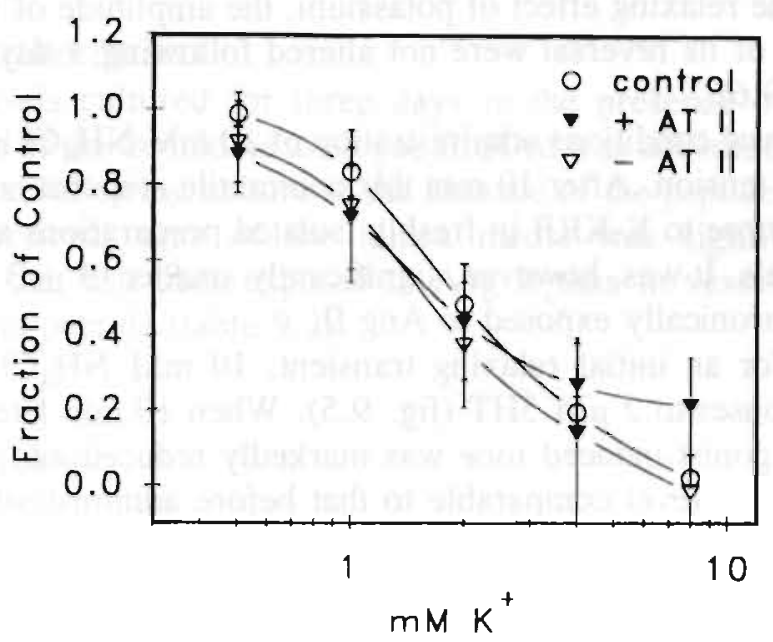

Figure 9.6 Concentration-response curves for the relaxing effect of potassium ions in renal artery segments that had been made to contract with $2 \mu \mathrm{M} 5 \mathrm{HT}$ and exposed to potassium-free solution for $10 \mathrm{~min}$. Data were expressed as fraction of the active tone prior to potassium removal and are shown as mean \pm SEM for freshly isolated preparations (O), cultured preparations $(\nabla)$ and vessels exposed to $1 \mu \mathrm{M}$ Ang II for 3 days $(\boldsymbol{\nabla})$.

\section{Alteration of arterial smooth muscle excitability}

Responses to K-KRB were not altered by culture or by 3 day exposure to Ang II (table 9.1). Also the amplitude of the maximal response to 5HT and the sensitivity to the indolamine were not altered following long term maintenance of the arterial preparations in vitro with or without continuous presence of Ang II (fig. 9.4). Furthermore, acute contractile responses to Ang II were not modified by culture or continuous exposure to Ang II (table 9.1). When cultured vessels were allowed $90 \mathrm{~min}$ to recover, the amplitude, time course and lack of reproducibility of responses to Ang II, were comparable to those in freshly isolated preparations (table 9.1).

In additional experiments, we indirectly addressed the possibility that sodium handling in arterial smooth muscle was altered. During contractions induced by $2 \mu \mathrm{M} 5 \mathrm{HT}$, effects of removal and readministration of extracellular potassium ions and effects of ammonium ions and their removal were evaluated. During $10 \mathrm{~min}$ of potassium-removal the amplitude of the 5HT- 
induced contraction increased moderately. Subsequent administration of potassium caused concentration dependent relaxations (fig. 9.5). As expected this relaxing effect was transient. The effect of potassium removal, the sensitivity for the relaxing effect of potassium, the amplitude of this effect and the time course of its reversal were not altered following 3 days of exposure to Ang II (fig. 9.6).

Under basal conditions administration of $10 \mathrm{mM} \mathrm{NH}_{4} \mathrm{Cl}$ induced a slow increase in wall tension. After $10 \mathrm{~min}$ this contractile response averaged $41 \pm$. $4 \%$ of the response to $\mathrm{K}-\mathrm{KRB}$ in freshly isolated preparations and $38 \pm 6 \%$ in cultured vessels. It was, however, significantly smaller $(9 \pm 3 \%)$ in arteries that had been chronically exposed to Ang II.

Except for an initial relaxing transient, $10 \mathrm{mM} \mathrm{NH}_{4} \mathrm{Cl}$ did not alter contractile responses to $2 \mu \mathrm{M} 5 \mathrm{HT}$ (fig. 9.5). When $10 \mathrm{~min}$ later, the $\mathrm{NH}_{4} \mathrm{Cl}$ was removed, agonist-induced tone was markedly reduced and then returned within minutes to a level comparable to that before administration of $\mathrm{NH}_{4} \mathrm{Cl}$ (fig. 9.5). In freshly isolated renal artery segments $(n=4)$, no recovery of tone was observed following removal of ammonium ions, when the protocol was performed in the presence of $1 \mathrm{mM}$ amiloride, an inhibitor of sodium-hydrogen exchange. Effects of $\mathrm{NH}_{4} \mathrm{Cl}$ on $5 \mathrm{HT}$-induced tone were comparable in freshly

Table 9.2 Effect of Ang II on growth responses in isolated arteries ${ }^{\mathrm{a}}$

Treatment

None $\quad$ Ang II

$\begin{array}{lcr}\text { BrdUrd (\%) } & 10.6 \pm 3.7 & 11.5 \pm 4.1 \\ \operatorname{CSA}\left(\mu \mathrm{m}^{2} \times 10^{-3}\right) & 65 \pm 2 & 81 \pm 6^{*}\end{array}$

${ }^{a}$ Renal artery segments that had been sympathectomized and denuded of endothelium were maintained during three days in culture medium containing $1 \mu \mathrm{g} / \mathrm{ml}$ indomethacin and $1 \mu \mathrm{M}$ BrdUrd with or without $1 \mu \mathrm{M}$ Ang II. Nuclear staining for BrdUrd was subsequently determined in the tunica media along with media cross sectional area (CSA). Data are shown as means \pm SEM $(n=6)$. "The effect of Ang II is statistically significant $(P * 0.05)$.

isolated preparations and segments cultured in absence or presence of Ang II. The amplitude of the transient relaxing effect of ammonium-removal was, 
however, significantly smaller in vessels that had been chronically exposed to Ang II $(-47 \pm 11 \%)$ than in either fresh vessels $(-85 \pm 3 \%)$ or vessels cultured without Ang II $(-71 \pm 8 \%)$.

\section{Structural alterations}

In vessels cultured for three days in the presence of Ang II nuclear incorporation of the thymidine analogue BrdUrd was not stimulated beyond the level observed following culture in the absence of the peptide (table 9.2). Yet, the cross sectional area of the tunica media was significantly larger in preparations that had been exposed to Ang II than in vessels cultured in the absence of the peptide (table 9.2).

\section{DISCUSSION}

Continuous exposure of isolated arterial smooth muscle to Ang II resulted in a progressive increase in tone. This long term contractile action developed several hours after the acute effect and was maintained for several days. Previously reported effects of Ang II on arterial smooth muscle growth (Berk et al, 1989a; Owens, 1989; Griffin et al, 1991; Turla et al, 1991) and on the production of autocrine vasoconstrictors (Dzau, 1988; Dohi et al, 1992) do not seem to participate in this long term contractile action of Ang II.

Recording of force development during arterial organ culture was used to address the possibility that Ang II induces besides its well known acute transient contractile action, also a long term tonic vasoconstriction. We have previously shown that arterial segments can be maintained for several days in culture without significant alteration of the vasoconstrictor action of K-KRB or 5HT (De Mey et al, 1989). Under these conditions significant DNA-synthesis can be detected. This mitogenic response in the isolated arterial wall can be stimulated by growth factors and serum and reduced by various pharmacologi$\mathrm{cal}$ agents and by the endothelium (De Mey et al, $1991^{\mathrm{c}}$ ). We reasoned that the approach could be used to monitor long term actions of agents and drugs on the structure and function of the arterial wall. The rat renal artery was selected for these studies because the rat renal vasculature transiently constricts upon acute exposure to Ang II (Collis and Vanhoutte, 1978; De Mey et al, 1989) and because stimulated mitogenic responses are more marked in renal arteries than in any other isolated rat artery evaluated so far (De Mey et al, $1991^{\mathrm{c}}$ ).

Continuous exposure of sympathectomized and denuded arterial segments to ATII resulted in a biphasic contractile response. The well known acute and highly transient contractile effect of the peptide was followed, after a latency of 12 hours, by a slow progressive increase in tone. Several possibilities were considered to explain the secondary slow contractile effect. 
Trophic Action. An increase in wall mass may increase wall tension. Ang II was observed to increase media cross sectional area without affecting DNA-synthesis. This is in line with the trophic action of the peptide on arterial smooth muscle cells during cell culture (Berk et al, 1989ª Owens, 1989; Turla et al, 1991). A major role of this process in the tension response to continuous Ang II is, however, unlikely. SNP and removal of the agonist reversed the response suggesting that it was functional rather than structural in origin.

Production of intra-arterial vasoconstrictor agents. Both in vivo and during cell culture, Ang II has been reported to stimulate the production of prostaglandins (Shebuski and Aiken, 1980; Dzau, 1988), some of which may induce contraction of vascular smooth muscle. More recently, Ang II has been observed to stimulate expression of the potent vasoconstrictor agent endothelin1 (Furchgott and Vanhoutte, 1989; Dohi et al, 1992). A contribution of these to the development of tonic tone during continuous exposure to Ang II seems unlikely in view of the observations in the presence of indomethacin and the endothelin antagonist BQ123. Furthermore bioassay experiments, in which conditioned culture media were screened for vasoconstrictor activity, failed to support the hypothesis of autocrine vasoconstrictor production. In the bioassay experiments, we deliberately used two types of preparations to sense vasoconstrictor activity (segments that had been continuously exposed to Ang II and segments not exposed to the peptide) to take into account that not only the mediator but also the appropriate receptors could be produced during continuous exposure to Ang II. Such a mechanism may be operative during stimulated production of platelet-derived-growth-factor (Terracio et al, 1988; Majesky et al, 1990) which can constrict the rat aorta (Berk et al, 1986).

Tachyphylaxis of Tachyphylaxis. Although most blood vessels and vascular beds constrict acutely in response to Ang II, these responses fade rapidly despite continuous presence of the agonist (Collis and Vanhoutte, 1978). In analogy to other "seven-transmembrane-domain-receptors" (Sibley and Lefkowitz, 1985) this has been attributed to receptor desensitization and internalisation. The possibility that also these processes would be susceptible to tachyphylaxis or that they would be counteracted by secondary stimulation of angiotensin-receptor synthesis (Inagami et al, 1992) does not seem to underlay the secondary slow contractile response to Ang II. The amplitude and time course of acute contractile responses to Ang II was comparable for arterial segments cultured in the absence of Ang II and for those that had been exposed to the peptide during 3 days and allowed to recover for $90 \mathrm{~min}$ in drug-free physiological salt solution.

The role of angiotensin-receptors in the maintenance of tonic tone in vessels continuously exposed to peptide is, however, not clear in view of the resistance of the response to reversal by Sar, an angiotensin-receptor antagonist that was used because of its non-selectivity for angiotensin-receptor subtypes 
(Inagami et al, 1992). Since freshly isolated and cultured vessels did not contract in response to up to $10 \mu \mathrm{M}$ Sar (unpublished observations), it is not likely that the result is complicate by partial agonist properties of the antagonist. In this respect, a role for intracellular angiotensin-bincling sites ( $R e$ et al, 1983), acted upon by Ang II during prolonged incubation, but not acutely accessible to Sar, may merit consideration.

Protein kinase $\mathbf{C}$ and ion handling. In isolated arterial smooth muscle cells, Ang II acutely influences phosphoinositide hydrolysis (Griendling et al, 1986, 1988), intracellular pH (Griendling et al, 1988) and cellular sodium handling (Kuriyama et al, 1988; Vallega et al, 1988). These processes are biphasic and interrelated (Griendling et al, 1988). Unlike stimulated production of inositol trisphosphate, the generation of diacylglycerol may be sustained for at least $30 \mathrm{~min}$. Continuous production of this natural activator of protein kinase $\mathrm{C}$ has been reported to depend on intracellular alkalinization secondary to Ang II-stimulated sodium-hydrogen exchange (Griendling et al, 1988). In turn, this exchange depends on the sodium gradient generated by the electrogenic sodium-potassium pump (Kuriyama et al, 1988). In theory, several aspects of this complex interaction observed during acute experimentation, could be involved in the tonic tone which we observed during continuous presence of Ang II. Stimulation of protein kinase C increases the concentration and efficacy of intracellular calcium which may account for the acute potentiating action of Ang II (Henrion et al, 1992) and could participate in the presently observed chronic effect. Chronic stimulation has, however, been observed to downregulate protein kinase $\mathrm{C}$. Yet, following 3 day exposure to Ang II no selective alteration of contractile responses to K-KRB or $5 \mathrm{HT}$ could be detected. Long term sodium loading of cells has been observed to upregulate the number of $\mathrm{Na}^{+}-\mathrm{K}^{+}$ATPase sites under certain conditions leading to subsequent normalization of the intracellular sodium concentration (Allen et al, 1989; Seidel et al, 1990). Following 3 day exposure to Ang II we found no alteration of potassium-induced relaxations, excluding major changes in electrogenic sodium transport. In view of this, sodium loading of Ang II-treated smooth muscle may result from increased membrane permeability and stimulated sodium-hydrogen exchange (Kuriyama et al, 1988; Vallega et al, 1988). Initially this will activate electrogenic sodium transport (Kuriyama et al, 1988; Allen et al, 1989) which may account for the long transition period between the acute and long term contractile action of Ang II. When ultimately established, the combination of elevated intracellular sodium and $\mathrm{pH}$ may promote contraction through depolarization, sodium-calcium exchange and increased calcium sensitivity of calmodulin and caldesmon (Aickin, 1986; Aalkjaer, 1990). Our observations in Ang II-treated vessels of reduced contraction in response to ammonium and reduced relaxation after ammoniumremoval may be in line with such a mechanism. This, however, remains to be 
firmly established through direct measurements of intracellular sodium concentration and $\mathrm{pH}$.

Tonic tone during continuous exposure to Ang II was significantly larger in denuded vessels than in vessels with endothelium. This adds to the protective role of the endothelium previously suggested by its antithrombotic properties, release of vasodilator agents and capacity to inhibit smooth muscle cell proliferation (Gryglewski et al, 1988; Furchgott and Vanhoutte, 1989; De Mey et al, $1991^{\mathrm{c}}$ ).

Although the mechanism remains to be established, our observations indicate that Ang II elicits besides its acute and transient vasoconstrictor action also a long-term tonic increase in arterial tone through a direct action on arterial smooth muscle function. It may be of interest to compare the role of this long-term functional action to that of acute and trophic effects of Ang II in pathological conditions that are characterized by increased supply of Ang II to the vascular wall or by altered responses to normal plasma levels of the peptide (Dzau, 1988; Berk et al, 1989 ; Resink et al, 1989 ${ }^{\mathrm{b}}$; Scott-Burden et al, 1989'; Martinez-Maldonado, 1991). Furthermore, the observed long-term vascular action may contribute to the hemodynamic changes observed in rats continuously treated with initially suppressor doses of Ang II. This intervention results in a slowly developing increase in blood pressure (Brown et al, 1981; Lever, 1986; Griffin et al, 1991) that can be prevented by a vasodilator such as hydralazine (Griffin et al, 1991) and can be partly reversed by discontinuation of the infusion (Brown et al, 1981). Extrapolation from the present in vitro finding to this or any other in vivo condition will, however, have to take into account not only the kinetics of acute and long-term responses but also their relative concentration dependencies and the potential inhibitory effect of endothelium on functional and structural responses of arterial smooth muscle.

In general, our findings indicate that long term actions of agents on the vasculature can not always be predicted by integration over time of their acute effects or by the algebraic sum of acute functional and slow structural phenomena. We previously reached a similar conclusion during comparison of the acute and long term actions of atrial natriuretic peptides on blood pressure (De Mey et al, 1987). 


\section{Chapter}

10

Conclusions and Outlook 


\section{CONCLUSIONS AND OUTLOOK}

Atherosclerosis and hypertension are characterized by an altered vascular structure (Folkow, 1982; Schwartz et al, 1986). Atherosclerotic lesions occur in the intima at sites of smooth muscle proliferation. The smooth muscle cells contribute to the lesion by proliferation and by synthesis of extracellular matrix. Within the vascular tree, large arteries and coronary arteries are affected to a larger extent by atherogenic conditions than the microcirculation. In hypertension, increased resistance is due to an increase in wall-to-lumen ratio. In large arteries this results from an increase in media mass as a result from smooth muscle cell hypertrophy/hyperploidy. In small arteries, however, sinooth muscle cell hyperplasia or remodeling of a normal media mass underlie the increased resistance in hypertension. Thus, different types of structural changes occur during these diseases, and various parts of the circulatory system differ in their susceptibility to modification.

The reasons for the different growth responses in these vascular diseases and their regional heterogeneity are poorly understood. Phenomena, such as regional differences in hemodynamics, nervous input and supply of exogenous mitogens, could play a role (Owens, 1988; Bevan and Tsuru, 1981). In addition, mechanisms that are endogenous to the vascular wall could also be involved, whereby ASMC phenotype and composition of the surrounding extracellular matrix are possibly important (Schwartz et al, 1986; ChamleyCampbell et al, 1979; Blank et al, 1988). Furthermore, ASMC and endothelial cells can produce factors that affect growth responses in an autocrine and paracrine manner (Davies, 1986; Walker et al, 1986; Sjölund et al, 1988; Staiano-Coico et al, 1988, De Met et al, $1991^{\mathrm{a}}$ ), that may be different between vascular beds and change with branching.

To obtain more insight in the mechanisms and factors that rule the heterogeneity of growth responses in the intact vascular wall, an arterial organ culture system was used in this thesis. First, growth responses of isolated and denuded rat renal arteries to serum were evaluated (Chapter 3). Two different types of growth responses were observed. The initial transient stimulation of DNA synthesis in the media is in agreement with observations in the arterial wall in response to elevated blood pressure and injury in vivo (McLean and Nakaone, 1974; Carlier et al, 1983; Loeb et al, 1986; Majesky et al, 1987). This stimulation of DNA synthesis was restricted to a fraction of the medial cells, i.e. not all smooth muscle cells participated. With time a second response was observed. It consisted of formation of new layers of cells outside the original vessel wall. At least part of these newly formed cells displayed smooth muscle-like properties. The maximal medial DNA synthesis was observed at 3 days of culture, whereas the appearance of the newly formed layers of cells at the outer edge of the vessel wall started at day 5 and increases markedly 
during 14 days of culture. During this short- and long-term arterial organ culture, neither the number of the cells in the media, the ploidy of the arterial nuclei nor medial CSA changed. Therefore, the connection between both types of growth responses may possibly be DNA synthesis of medial ASMC followed by cell division and migration of cells to the outside of the original vessel wall with subsequent proliferation of the latter migrated cells. Thus, this model of arterial organ culture is suited to study these important growth responses occurring in atherosclerosis and hypertension, i.e. DNA synthesis in the media (atherosclerosis, hypertension) and migration and proliferation of cells (atherosclerosis).

The extent of medial DNA synthesis and formation of new layers of cells differed quantitatively between arteries of different anatomical origin (Chapter 4). Again, only in part of the arterial media cells, DNA synthesis could be stimulated. On the other hand, proliferation rates of large artery smooth muscle cells isolated from different parts of the circulatory system, did not differ in cell culture. This indicates that phenomena within the arterial media modulate the growth responsiveness of the arterial smooth muscle cells. In this respect, the suggested presence of a mixture of ASMC subtypes, which display different growth characteristics (Chapter 4), indicates that growth-resistant and growthprone ASMC could be heterogeneously distributed over the arterial tree. In addition to this, the endothelium has been observed to modulate medial DNA synthesis differently, depending on the anatomical location of the isolated vessel. Thus, regional heterogeneity of endothelial cells and smooth muscle cell subtypes may contribute to regional heterogeneity of growth responses in the vascular wall.

The endothelium was found to be a strong inhibitor of medial DNA synthesis in arterial organ culture (Chapter 5). In hypertension, endothelial dysfunction with respect to arterial tone has been described extensively (Furchgott and Vanhoutte, 1989; De Mey and Gray, 1985; Lüscher, 1990). This endothelial dysfunction is characterized by an imbalance between contracting and relaxing endothelium-derived factors. Unlike this reduction in endothelium-dependent relaxation in hypertension, endothelium-dependent inhibition of arterial DNA synthesis is not uniformly reduced following hypertension (Chapter 5). In arteries of all strains and models of hypertension (young SHR, aorta-coarcted WKY, and TGR), except for adult SHR, DNA synthesis was more marked in the absence of the endothelium than in its presence. The differential behavior of adult SHR arteries may, therefore, be independent of blood pressure. The mechanism underlying this altered endothelial inhibition of DNA synthesis, is not clear.

The mediators that may be involved in arterial growth control have been extensively studied in cell culture and following arterial injury in vivo (Schwartz et al, 1986; Chamley-Campbell et al, 1979; Clowes et al, 1983b 
Jackson and Schwartz, 1992). From these studies, it is suggested that ASMC and endothelial cells can produce mediators that interfere with arterial growth responses. One of these growth affecting mediators, bFGF, had a marked stimulatory effect on medial DNA synthesis in organ culture, but not on proliferation of extramedial cells (Chapter 6). This observation is in agreement with observations following balloon catheter injury in vivo, whereby bFGF participates in the stimulation of medial DNA synthesis but not in the proliferation of neointimal cells (Olson et al, 1992). The PDGF's are powerful mitogens for isolated cells (Ross et al, 1986) but had no effect on DNA synthesis and cellular proliferation in arterial organ culture (Chapter 6). These observations give strength to the suggestion that PDGF's may act primarily as chemotactic agents (Jawien et al, 1992) in the arterial wall in vivo. In analogy with its effects on media contractility, the endothelium has the potential to both inhibit (IL-1, TGF- $\beta$ ) and stimulate (bFGF, ET-1) growth responses in the underlaying smooth muscle (Chapter 6 ). However, only TGF- $\beta$ reduced medial DNA synthesis during maximal stimulation by serum. On the other hand, only bFGF increased medial DNA synthesis in the presence of endothelium. These observations suggest that the balance of TGF- $\beta$ and bFGF may be important in endothelium-dependent arterial growth control.

It is well established that the renin-angiotensin system and sympathetic nerves are involved in the development of hypertension and in the structural changes that are associated with it (Head, 1991; Lee and Smeda, 1985; Sahlgren et al, 1986; Lever, 1986). In the SHR an increased sympathetic activity is suggested to play an important role in the development of high blood pressure. Regional heterogeneity of arterial structural changes may result from a non-uniform density of sympathetic nerves along the arterial tree (Abboud, 1982). The fact that Ang II increases sympathetic nerve activity centrally and peripherally (Severs and Daniels-Severs, 1973; Zimmerman, 1978) and potentiates ASMC responses to alpha-adrenoceptor stimulation (Vanhoutte et al, 1981) indicates the interrelationship of the renin-angiotensin system and the sympathetic nervous system. However, in vivo infusions of Ang II and PHE in young rats showed that growth responses differed between Ang II, PHE and SHR, between types of blood vessels, and were different between subtypes of smooth muscle cells within an arterial segment (Chapter 8). These observations suggest that: (i) arterial wall hypertrophy induced by vasoconstrictors is not only due to elevated blood pressure: (ii) regional heterogeneity of trophic responses to Ang II or PHE is more likely related to regional differences in receptor subtypes than in sympathetic nerve density; and (iii) arterial growth responses depend on the ASMC subtype composition of the media.

Infusions of low doses of Ang II in vivo results in a slowly developing progressive increase in blood pressure (Lever, 1986). Yet, this slow-pressor action of Ang II may have not only a structural but also a functional basis 
(Chapter 8). Ang II induces in isolated arterial smooth muscle, besides its wellknown acute contractile effect and its trophic action, a long-term tonic increase in tone (Chapter 9). The mechanism underlying this slow increase in tone may involve altered cellular handling of hydrogen ions (Chapter 9). This long-term tonic increase in tone was markedly reduced by the endothelium.

In summary, ASMC growth responses are heterogenous throughout the vascular tree. The reasons for the regional heterogeneity are manyfold and include (1) the different endothelial modulation of arterial growth responses along the vascular tree; (2) the ASMC subtype composition of the arterial media, and (3) regional heterogeneity of arterial receptor subtypes. Whether an imbalance of production and actions of intravascular growth inhibitors and mitogens such as TGF- $\beta$ and $b F G F$ is responsible for the regional heterogeneity of growth responses deserves more attention in the future. Knowledge about the intra-arterial heterogeneity with respect to subsets of ASMC and receptor subtypes is also extremely important to predict arterial growth responses. This taken together, raises also the possible relevance for the development of novel drugs that prevent/reverse specific structural changes at specific locations occurring in vascular diseases. In addition, we have to keep in mind that not only structural but also slow functional mechanisms may be important in the development of hypertension. 


\section{REFERENCES}

Aalkjaer C, Heagerty AM, Petersen KK, Swales JD, Mulvany MJ: Evidence for increased media thickness, increased neuronal amine uptake, and depressed excitation-contraction coupling in isolated resistance vessel from essential hypertensives. Circ Res $1987^{\prime} ; 61: 181-186$.

Aalkjaer C, Heagerty AM, Swales JD, Thurston H: Endothelium-dependent relaxation in human subcutaneous resistance vessels. Blood vessels $1987^{\mathrm{b}} ; 24: 85-88$.

Aalkjaer C, Eiskjaer H, Mulvany MJ, Jespersen B, Kjaer T. Abnormal structure and function of isolated subcutaneous resistance vessels from essential hypertensive patients despite antihypertensive treatment. J Hypertension 1989;7:305-310.

Aalkjaer C: Regulation of intracellular $\mathrm{pH}$ and its role in vascular smooth muscle function. J Hypertension 1990;8:197-206.

Abboud FM: The sympathetic nervous system in hypertension. Clin Exp Hypertension $1984 ; 6: 43-60$.

Adair TH, Gay WJ, Montani JP: Growth regulation of the vascular system: evidence for a metabolic hypothesis. Am J Physiol 1990;259:R393-R404.

Aickin CC: Intracellular $\mathrm{pH}$ regulation by vertebrate smooth muscle. Ann Rev Physiol 1986;48:349-361.

Allen JC, Navran SS, Seidel CL, Dennison DK, Amann JM, Jemelka SK: Intracellular $\mathrm{Na}^{+}$ regulation of $\mathrm{Na}^{+}$pump sites in cultured vascular smooth muscle cells. Am J Physiol 1989;256:C786-C792.

Antonaccio MJ, Kerwin L: Pre-and postjunctional inhibition of vascular sympathetic function by captopril in SHR: implication of vascular angiotensin II in hypertension and antihypertensive actions of captopril. Hypertension 1981;3(suppl I):54-62.

Aprigliano $\mathrm{O}$, Hermsmeyer $\mathrm{H}$ : In vitro denervation of the portal vein and caudal artery of the rat. J Pharmacol Exp Ter 1976;198:568-577.

Assoian RK, Fleurdelys BE, Stevenson HC, Miller PJ, Madtes DK, Raines EW, Ross R, Sporn MB: Expression and secretion of type B transforming growth factor by activated human macrophages. Proc Natl Acad Sci USA 1987;84:6020-6024.

Assoian RK, Sporn MB: Type B transforming growth factor in human platelets: release during platelet degranulation and action on vascular smooth muscle cells. J Cell Biol 1986; 102:1217-1223.

Azuma $\mathrm{H}$, Ishikawa M, Sekizaki S: Endothelium-dependent inhibition of platelet aggregation. Br J Pharmacol 1986;88:411 415.

Baenziger NL, Fogerty FJ, Mutz LF, Chemuta LF: Regulation of histamine-mediated prostacyclin synthesis in cultured human vascular endothelial cells. Cell 1981;24:915923.

Barrett TB, Sampson P, Owens GK, Schwartz SM, Bendilt EP: Polyploid nuclei in human artery wall smooth muscle cells. Proc Natl Acad Sci USA 1983;80:882-885.

Bauch H-J, Grünwald J, Vischer P, Gerlach U, Hauss WH: A possible role for catecholamines in atherogenesis and subsequent complications of atherosclerosis. Exp Pathol 1987;31:193-204.

Baumbach GL. Heistad DD: Remodeling of cerebral arterioles in chronic hypertension. Hypertension 1989;13:968-972.

Beilin LJ, Vandongen R, Arkwright PD, Davidson L: Adrenal and sympathetic nervous activity in subjects with "low" and "high" normal blood pressure. J Hypertension $1983 ; 1: 13-18$. 


\section{REFERENCES}

Benditt EP, Benditt JM. Evidence for a monoclonal origin of the human atherosclerotic plaque. Proc Nat Acad Sci USA 1973;70:1753-1756.

Berk BC, Alexander RW, Brock TA, Gimbrone MA, Webb RC: Vasoconstriction: a new activity for platelet-derived growth factor. Science 1986;232:87-90.

Berk BC, Alexander RW: Vasoactive effects of growth factors. Biochem Pharmacol 1989;38(2):219-225.

Berk BC, Vekshtein V, Gordon HM, Tsuda T: Angiotensin II-stimulated protein synthesis in cultured vascular smooth muscle cells. Hypertension 1989; $13: 305-314$.

Berk BC, Vallega G, Muslin AJ, Gordon HM, Canessa M, Alexander W: Spontaneously hypertensive rat vascular smooth muscle cells in culture exhibit increased growth and $\mathrm{Na}^{+} / \mathrm{H}^{+}$exchange. $\mathrm{J}$ Clin Invest $1989^{\mathrm{b}} ; 83: 822-829$.

Berk BC, Elder E, Mitsuka M: Hypertrophy and hyperplasia cause differing effects on vascular smooth muscle cell $\mathrm{Na}^{+} / \mathrm{H}^{+}$exchange and intracellular $\mathrm{pH}$. J Biol Chem 1990;265(32): 19632-19637.

Bevan RD, Tsuru H: Long-term denervation of vascular smooth muscle causes not only functional but structural damage. Blood Vessels 1979;16:109-112.

Bevan RD, Tsuru H: Functional and structural changes in the rabbit ear artery after sympathetic denervation. Circ Res 1981;49:478-485.

Bing RF, Russell GI, Swales JD, Thurston H: Effect of 12 hour infusion of saralasin or captopril on blood pressure in hypertensive conscious rats. Relationship to plasma renin, duration of hypertension and effect of unclipping. J Lab Clin Med 1981;98:302310.

Björkerud S, Bondjers G: Arterial repair and atherosclerosis after mechanical injury: tissue response after induction of a large superficial transverse injury. Atherosclerosis 1973;18 (suppl 5):235-255.

Björkerud S, Bondjers G: Repair responses and tissue lipid after experimental injury to the artery. Ann N Y Acad Sci 1976;175:180-198.

Black MJ, Campbell JH, Campbell GR: Does smooth muscle cell polyploidy occur in resistance vessels of spontaneously hypertensive rats. Blood Vessels 1988;25:89-100.

Black MJ, Adams MA, Bobik A, Campbell JH, Campbell GR: Effect of enalapril on aortic smooth muscle cell polyploidy in the spontaneously hypertensive rat. J Hypertension 1989;7:997-1003.

Blaes N, Boissel JP: Growth-stimulating effect of catecholamines on rat aortic smooth muscle cells in culture. J Cell Physiol 1983;116:167-172.

Blank RS, Thompson MM, Owens GK: Cell cycle versus density dependence of smooth muscle actin expression in cultured rat aortic smooth muscle cells. J Cell Biol 1988; 107:299-306.

Bobik A, Grooms A, Millar JA, Mitchell A, Grinpukl S: Growth factor activity of endothelin on vascular smooth muscle. Am J Physiol 1990;258:C408-C415.

Bohlen $\mathrm{H}$, Lohach $\mathrm{D}$ : In vivo study of microvascular wall characteristics and resting control of young and mature spontaneously hypertensive rats. Blood Vessels 1978;15:322-330.

Bondjers G, Björnheden T: Experimental atherosclerosis induced by mechanical trauma in rats. Atherosclerosis 1970;12:301-306.

Bondjers G, Glukhova M, Hansson GK, Postnov YV, Reidy MA, Schwarz SM: Hypertension and atherosclerosis. Cause and effect, or two effects with one unknown cause? Circulation 1991;84 (suppl 6):2-16.

Bonin PD, Fici GJ, Singh JP: Interleukin-1 promotes proliferation of vascular smooth muscle 


\section{REFERENCES}

cells in coordination with PDGF or a monocyte derived growth factor. Exp Cell Res 1989; 181:475-482.

Boonen HCM, De Mey JGR: Increased calcium sensitivity in isolated resistance arteries from spontaneousily hypertensive rats: effects of dihydropyridines. Eur J Pharmacol 1990; 179: 403-412.

Boonen HCM, Schiffers PMH, Fazzi GE, Daemen MJAP, De Mey JGR: DNA synthesis in isolated arteries. Kinetics and structural consequences. Am J Physiol 1991;260:H210$\mathrm{H} 217$.

Boulanger CM, Lüscher TF: Release of endothelin from the porcine aorta: Inhibition by endothelium-derived nitric oxide. J Clin Invest 1990;85:587-590.

Bowen-Pope DF, Ross R: Platelet-derived growth factor.II. Specific binding to cultured cells. J Biol Chem 1982;257:5161-5171.

Boyer JL, Hepler JR, Harden TK. Hormone and growth factor receptor-mediated regulation of phospholipase C activity. TiPS 1989;10:360-364.

Bradford MA: A rapid and sensitive method for quantitation of microgram quantities of protein utilizing the principle of protein-binding. Anal Biochem 1976;72:248-259.

Bravo R, Frank R, Blundell PA, Macdonald-Bravo H: Cyclin/PCNA is the auxiliary protein of DNA polymerase-delta. Nature 1987;326:515-517.

Brown AJ, Casals-Stenzel J, Gofford S, Lever AF, Morton JJ: Comparison of fast and slow pressor effects of ATIl in the conscious rat. Am J Physiol 1981;241:H381-H388.

Bubin D, Pratt RE, Cooke JP, Dzau VJ: Endothelin, a potent vasoconstrictor, is a vascular smooth muscle mitogen. J Vasc Pharmacol 1989;1:150-154.

Burgess WH, Maciag $\mathrm{T}$. The heparin-binding (fibroblast) growth factor family of proteins. Ann Rev Biochem 1989;58:575-606.

Busse R, Foerstermann U, Matsuda H, Pohl U: The role of prostaglandins in the endothelium-mediated vasodilator response to hypoxia. Pflügers Arch 1984;401:77-83.

Campbell-Boswell M, Robertson A: Effects of angiotensin II and vasopressin on human smooth muscle cells in vitro. Exp Mol Pathol 1981;35:265-276.

Carlier P, Rorive R, Barbason H: Kinetics of proliferation of rat aortic smooth muscle cells in Goldblatt one-kidney, one-clip hypertension. Clin Sci 1983;65:351-357.

Carlier PG, Rorive GL: Pathogenesis and reversibility of the aortic changes in experimental hypertension. J Cardiovasc Pharmacol 1985;7 (suppl):46-51.

Castellot JJ, Addonizzio ML, Rosenberg R, Karnovsky MJ: Cultured endothelial cells produce a heparin-like inhibitor of smooth muscle cell growth. I Clin Invest $1981 ; 90: 372-379$.

Castellot JJ, Wright TC, Karnovsky MJ: Regulation of smooth muscle cell growth by heparin and heparin sulphates. Sem Thom Hem 1987;13:487-503.

Chamley-Campbell J, Campbell GR, Ross R: The sinooth muscle cell in culture. Physiol Rev $1979 ; 59: 1-61$.

Chamley-Campbell J, Campbell GR, Ross R: Phenotype-dependent response of cultured aortic smooth muscle to serum mitogens. J Cell Biol 1981;89:379-383.

Champlain de $\mathbf{J}$ : Pre- and postsynaptic adrenergic dysfunctions in hypertension. J Hypertension 1990; 8 (suppl 7):77-85.

Chardonnens D, Lang U, Capponi AM, Vallotton MB: Comparison of the effects of angiotensin II and vasopressin on cytosolic free calcium concentration, protein kinase $\mathrm{C}$ activity, and prostacyclin production in cultured rat aortic smooth muscle cells. J Cardiovasc Pharmacol 1989;14(suppl 6):39-44.

Chiu AT, Roscoe WA, McCall D, Timmermans PBMWM: Angiotensin II-1 receptors 
mediate both vasoconstrictor and hypertrophic responses in rat aortic smooth muscle cells. Receptor 1991;1:133-140.

Chobanian AV, Brecher PI, Haudenschild CC: Effects of hypertension and of antihypertensive therapy on atherosclerosis. Hypertension 1986;89 (suppl 1):15-21.

Chobanian AV, Lichtenstein AH, Schwartz JH, Hanspal J, Brecher P: Effects of deoxycorticosterone/salt hypertension on cell ploidy in rat aortic smooth muscle cells. Circulation 1987;75 (suppl I): 102-106.

Chobanian AV, Haudenschild CC, Nickerson C, Drago R: Antitherogenic effect of captopril in the Watanabe heritable hyperlipidemic rabbit. Hypertension 1990;15:327-331.

Christensen HRL, Nielsen H, Christensen KL, Baandrup U, Jespersen LT: Long-term effects of an angiotensin converting enzyme inhibitor in spontaneously hypertensive rats: is there a role for vascular structure? J Hypertension 1988;6 (suppl 3):27-31.

Christensen KL, Jespersen LT, Mulvany MJ: Development of blood pressure in spontaneously hypertensive rats after withdrawal of long-term treatment related to resistance vessel structure. J Hypertension 1989;7:83-90.

Clemmons DR, Underwood LE, Chatelain PG, van Wijk J: Liberation of immunoreactive somatomedin-C from its binding proteins by proteclytic enzymes and heparin. J Clin Endocrinol Metab 1983;56:384-389.

Clowes AW, Clowes MM: Influences of chronic hypertension on injured and uninjured arteries in spontaneously hypertensive rats. Lab Invest 1980;43:535-541.

Clowes AW, Reidy MA, Clowes MM: Kinetics of cellular proliferation after arterial injury. I. Smooth muscle growth in the absence of endothelium. Lab Invest $1983^{\text {nt }} ; 49: 327-333$.

Clowes AW, Reidy MA, Clowes MM: Mechanism of stenosis after arterial injury. Lab Invest $1983^{\mathrm{b}} ; 49: 208-215$.

Clowes AW, Clowes MM, Fingerly J, Reidy MA: Kinetics of cellular proliferation after arterial injury. V. Role of acute distension inn the induction of simooth muscle proliferation. Lab Invest 1989;60:360-364.

Clozel JP, Kuhn H, Hefti F: Effects of cilazapril on the cerebral circulation in spontaneously hypertensive rats. Hypertension 1989;14:645-651.

Cocks TM, Angus JA: Endothelium-dependent relaxations of coronary arteries by noradrenaline and serotonin. Nature 1983;305:627-630.

Cocks TM, Angus JA, Campbell JH et al: Release and properties of endothelium-derived relaxing factor (EDRF) from endothelial cells in culture. J Cell Physiol 1985;123:310320.

Collis MG, Vanhoutte PM: Vascular reactivity of isolated perfused kidneys from male and female spontaneously hypertensive rats. Cir Res 1977;41:759-767.

Collis MG, Vanhoutte PM: Increased renal reactivity to angiotensin but not to nerve stimulation or exogenous norepinephrine in renal hypertensive rats. Circ Res 1978;43:544-552.

Collis MG, De Mey JGR, Vanhoutte PM: Enhanced release of noradrenalin in the kidney of the young spontaneous hypertensive rat. Clin Sci 1979;7:233-234.

Collis MG, De Mey JGR, Vanhoutte PM: Renal vascular activity in the young spontaneously hypertensive rat. Hypertension 1980;2:45-52.

Coughlin S, Escobedo JA, Williams LT: Role of phosphatidylinositol kinase in PDGF receptor signal transduction. Science 1989;243:1191-1194.

Cuevas P, Carceller F, Ortega S, Zazo M, Nieto I, Giménez-Gallego G: Hypotensive activity of fibroblast growth factor. Science 1991;254:1208-1210.

Daemen M, Lombardi D, Goessens M, Bosman F, Schwartz S: Comparison between 
continuous S-phase labeling with bromodeoxyuridine (BrdU) and ${ }^{3} \mathrm{H}$-thynidine $\left({ }^{3} \mathrm{HT}\right)$ in the balloon injured rat aorta (abstract. Proceedings of the 30th Dutch Federation Meeting 1989:122.

Daemen MJ, Lombardi DM, Bosman FT, Schwartz SM: Angiotensin II induces smooth muscle cell proliferation in the normal and injured rat arterial wall. Circ Res 1991;68:450-456.

Dahlöf B: Factors involved in the pathogenesis of hypertensive cardiovascular hypertrophy. Drugs 1988;35 (suppl 5):6-26.

D'Amore PAD, Thompson RW: Mechanisms of angiogenesis. Ann Rev Physiol 1987;49:453-465.

Davies PF: Vascular cell interactions with special reference to the pathogenesis of atherosclerosis. Lab Invest 1986;55:5-24.

Davies PF, Remuzzi A, Gordon EJ, De Wey CF, Gimbrone MA. Turbulent fluid shear stress induces vascular endothelial cell turnover in vitro. Proc Natl Acad Sci USA 1986;83:2114-2117.

De Mey J, Vanhoutte PM: Uneven distribution of postjunctional alpha-1 and alpha-2 like adrenoceptors in the canine arterial and venous smooth muscle. Circ Res 1981;48:875884.

De Mey J, Vanhoutte PM: Role of $\mathrm{Na}^{+}, \mathrm{K}^{+}$-ATPase in the vasodilator response to acetylcholine, In: Vanhoutte PM, Leusen I (eds): Vasodilation, New York, Raven Press, $1981^{\text {b }}$, pp 331-337.

De Mey JG, Claeys M, Vanhoutte PM: Endothelium-dependent inhibitory effects of acetylcholine, adenosine diphosphate, thrombin and arachidonic acid in the canine femoral artery. J Pharm Exp Ther 1982;222:166-173.

De Mey JGR, Vanhoutte PM: Heterogeneous behavior of the canine arterial and venous wall: importance of the endothelium. Circ Res 1982; $53: 439-447$.

De Mey JGR, Vanhoutte PM: $\mathrm{Na}^{+}-\mathrm{K}^{+}$exchanges in canine arterial and venous smooth muscle. Am J Physiol 1982 ; 243:H551-H559.

De Mey JG, Vanhoutte PM: Anoxia and endothelium-dependent reactivity in canine femoral artery. J Physiol 1983;335:65-74.

De Mey JGR, Brutsaert DL: Mechanical properties of resting and active isolated coronary arteries. Circ Res 1984;55:1-9.

De Mey JG, Gray SD: Endothelium-dependent reactivity in resistance vessels. Progress in applied microcirculation 1985;8:181-187.

De Mey JGR, Lenaers A, Defreyn G, Calderon P, Roba J: Arterial reactivity, blood pressure, and plasma levels of atrial natriuretic peptides in normotensive and hypertensive rats: effects of acute and chronic administration of atriopeptin III. Cariovasc Pharmacol 1987;9:525-535.

De Mey JGR, Uitendaal MP, Boonen HCM, Vrijdag MJJf, Daemen MJAP, StruykerBoudier HAJ: Acute and long-term effects of tissue culture on contractile reactivity in renal arteries of the rat. Circ Res 1989;65:1125-1135.

De Mey JGR, Dijkstra EH, Vrijdag MJJF: Endothelium reduces DNA synthesis in isolated arteries. Am J Phys 1991;260:H1128-H1134.

De Mey JGR, Dijkstra E, Fazzi G, Janssen G: Growth responses in the arterial wall. Bas Res Cardiol 1991 $; 86$ (suppl. 1):13-17.

De Mey JGR, Uitendaal MP, Boonen HCM, Schiffers PMH, Fazzi GE: Growth responses in isolated elastic, muscular and resistance-sized arterial segments of the rat. Blood Vesssels $1991^{\text {c }} ; 28: 372-385$. 


\section{REFERENCES}

De Mey JGR, Daemen MJAP, Boonen HCM, Bosman FT, Dijkstra EH, Fazzi GE, Janssen GMJ, Schiffers PMH, Struijker-Boudier HAJ, Vrijdag MJJF: In vivo DNA synthesis is not uniformly increased in arterial smooth muscle of young spontaneously hypertensive rats. J Hypertension 1991 ;9:695-701.

De Mey JGR, Schiffers PMH: Effects of the endothelium on growth responses in arieries. J Cardiovasc Pharmacol 1993;21 (suppl 1):S22-S25.

DiCorletto PE, Gajduzek CM, Schwartz SM, Ross R: Biochemical properties of the endothelium-derived growth factor: comparison to other growth factors. J Cell Physiol 1983;114:339-348.

DiCorletto PE, Fox PL: Growth factor production by endothelial cells. In: Ryan U (ed): Endothelial Cells, Bocan Raton Florida, CRC Press, 1988: pp51-61.

Dohi Y, Hahn AWA, Boulanger CM, Bühler FR, Lüscher TF: Endothelin stimulated by ATII augments contractility of spontaneously hypertensive rat resistance arteries. Hypertension 1992;19:131-137.

Dominiczak AF, Bohr DF: Vascular smooth muscle and hypertension. J Hypertension 1989;7 (suppl 4):107-115.

Donohue SJ, Head RJ, Stitzel RE: Elevated nerve growth factor levels in young spontaneously hypertensive rats. Hypertension 1989;14:421-426.

Dubin D, Pratt RE, Cooke JP, Dzau VJ: Endothelin, a potent vasoconstrictor, is a vascular smooth muscle mitogen. J Vasc Med Biol 1989;1:150-154.

Dudley DT, Panek RL, Major TC, Lu TC, Bruns RF, Klinkefus BA, Hodges JC, Weishaar RE: Subclasses of ATII binding sites and their functional significance. Mol Pharmacol 1990;38:370-377.

Dumout JE, Jaumiaux JC, Roger PP: The cyclic AMP-mediated stimulation cell proliferation. TIPS 1989;14:67-71.

Dzau VJ: Significance of the vascular renin-angiotensin pathway. Hypertension 1986;8:553559.

Dzau VJ: Tissue renin-angiotensin system: physiologic and pharmacologic implications. Circulation 1988;11:I1-I74.

Egan B, Panis R, Hinderliter A, Schork N, Julius S: Mechanisms of increased alphaadrenergic vasoconstriction in human essential hypertension. J Clin Invest 1987;80:812817.

Epstein FH: The epidemiology of essential hypertension. In: Handbook of hypertension. Clinical aspects of essential hypertension (ed: Robertson JIS). Elsevier, Amsterdam, 1983:1-21.

Evenwel RT, Kasbergen CM, Struyker-Boudier HAJ: Central and regional hemodynamics and plasma volume distribution during the development of spontaneous hypertension in rats. Clin Exp Hypertens [A] 1983;5:1511-1536.

Fager G, Hansson Gk, Gown AM, Larson DM, Shall O, Bondjers G: Human arterial smooth muscle cells in culture: inverse relationship between proliferation and expression of contractile proteins. In Vitro Cell Dev Biol 1989;25:51 1-520.

Feleton M, Vanhoutte PM: Endothelium-dependent hyperpolarization of canine coronary smooth muscle. Br J Pharmacol 1988;93:515-524.

Fingerle J, Kraft T: The induction of smooth muscle cell proliferation in vitro using an organ culture system. Int Angiol 1987;6:65-72.

Flavahan NA, Vanhoutte PM: Alpha-1-adrenoceptor subclassification in vascular smooth muscle. TIPS 1986; 7:347-349.

Folkow B: Physiological aspects of primary hypertension. Physiol Rev 1982; 62:347-504. 
Folkow B: "Structural factor" in primary and secondary hypertension. Hypertension 1990;16:89-101.

Fox PL, DiCorletto PE: Modified low density lipoproteins suppress production of plateletderived growth-like protein by cultured endothelial cells. Proc Natl Acad Sci USA 1986;83:4774-4778.

Francos JA, Eskin SG McIntyre LV, Ives LV: Flow effects on prostacyclin production by cultured human endothelial cells. Science 1985;227:1477-1479.

Freslon JJ, Giudicelli JF: Compared myocardial and vascular effects of captopril and hydralazine during hypertension development in spontaneously hypertensive rats. $\mathrm{Br}$ J Pharmacol 1983;80:533-543.

Furchgott RF: The role of endothelium in the response of vascular smooth muscle to drugs. Ann Rev Pharmacol Toxicol 1984;241:175-197.

Furchgott RF, Vanhoutte PM: Endothelium-derived relaxing and contracting factors. Faseb J 1989;3:2007-2018.

Gabbiani G, Glemes G, Guelpa C: Morphologic and functional changes of the aortic intima during experimental hypertension. Am J Pathol 1979;96:399-422.

Gajdusek C, DiCorletto, Ross R, Schwartz SM: An endothelial cell-derived growth factor. J Cell Biol 1980;85:467-472.

Gajdusek C, Caron S, Ross R, Nawroth P, Stern D: Activation of coagulation releases endothelial cell mitogens. J Cell Biol 1986;103:419-428.

Gajdusek CM, Caron S: Injury-induced release of basic fibroblast growth factor from bovine aortic endothelium. J Cell Physiol 1989;139:570-579.

Geisterfer AAT, Peach MJ, Owens GK: Angiotensin II induces hypertrophy, not hyperplasia, of cultured rat aortic smooth muscle cells. Circ Res 1988;62:749-756.

Geisterfer AAT, Owens GK: Arginine vasopressin-induced hypertrophy of cultured rat aortic smooth muscle cells. Hypertension 1989;14:413-420.

Gibbons GH, Pratt RE, Dzau VJ: Vascular smooth muscle cell hypertrophy vs. hyperplasia. Autocrine transforming growth factor beta- 1 expression determines growth response to angiotensin II. J Clin Invest 1992;90:456-461.

Gimenez-Gallego G, Rodbey J, Bennett C, Rios-Candelore M, DiSalvo J, Thomas KA: Brain-derived acidic fibroblast growth factor: complete amino acid sequence and homologics. Science 1985:230:1385-1388.

Glagov S, Giddens DP, Zarins C.K: Micro-architecture and composition of artery walls: relationship to location, diameter and the distribution of mechanical stress. J Hypertension 1992;10:S101-S104.

Goldblatt H, Lynch J, Hanzel RF, Summerville WW: Studies on experimental hypertension.1. The production of persistent elevation of systolic blood pressure by means of renal ischaemia. J Exp Med 1984;59:347-378.

Goldstein DS: Plasma catecholamines in essential hypertension: an analytical review. Hypertension 1983;5:86-99.

Goodman LV, Majack RA: Vascular smooth muscle cells express distinct transforming growth factor- $\beta$ receptor phenotypes as a function of cell density in culture. $J$ Cell Chem 1989;264:5241-5244.

Gordon D, Mohai LG, Schwartz SM: Induction of polyploidy in cultures of neonatal rat aortic smooth muscle cells. Circ Res 1986;59:633-644.

Gospodarowicz D, Hirabayashi K, Giguère L, Tauber J-P: Factors controlling the proliferative rate, final cell density, and life span of bovine vascular smooth muscle cells in culture. J Cell Biol 1981;89:568-578. 


\section{REFERENCES}

Gospodarowicz D, Ferrera N, Haaparanta T, Neufeld G: Basic fibroblast growth factor: Expression in cultured bovine vascular smooth muscle cells. Eur J Cell Biol 1988;46:144-151.

Gow BS: Circulatory correiates: vascular impedance, resistance, and capacity. In: The handbook of physiology. Sec. 2. The cardiovascular system. Vol II. Vascular smooth muscle edited by Bohr DF, Somlyo AP, Sparks HV. Bethesda, Maryland: American Physiological Society, 1980, pp 354-408.

Gown AM, Vogel AM, Gordon AM, Lu PL: A smooth muscle-specific monoclonal antibody recognizes smooth muscle actin isoenzymes. J Cell Biol 1985;100:807-813.

Grag UC, Hassid A. Nitric oxide generating vasodilators and 8-bromo-cyclic guanosine monophosphate inhibit mitogenesis and proliferation of cultured rat vascular smooth muscle cells. J Clin Invest 1989;83:1774-1777.

Gratzner G: Monoclonal antibody to 5-bromo and 5-iodo-deoxyuridine: a new reagent for detection of DNA replication. Science 1982;128:474-475.

Gray SD: Spontaneous hypertension in the neonatal rat: Clin Exp Hypertension 1984; A6(4):755-781.

Griendling KK, Rittenhouse SE, Brock TA, Ekstein LS, Gimbrone MA, Alexander RW: Sustained diacylglycerol formation from inositol phospholipids in ATII-stimulated vascular smooth muscle cells. J Biol Chem 1986;261:5901-5906.

Griendling KK, Berk BC, Alexander RW: Evidence that $\mathrm{Na}^{+} / \mathrm{H}^{+}$exchange regulates ATIIstimulated diacylglycerol accumulation in vascular smooth muscle cells. J Biol Chem 1988;263:10620-10624.

Griendling KK, Tsuda T, Berk BC, Alexander RW: Angiotensin II stimulation of vascular smooth muscle. J Cardiovasc Pharmacol 1989;14(suppl 6):27-33.

Griffin SA, Brown WCB, MacPherson F, McGrath JC, Wilson VG, Korsgaard N, Mulvany MJ, Lever AF: ATII causes vascular hypertrophy in part by a non-pressor mechanism. Hypertension 1991;17:626-635.

Grotendorst GR, Chang T, Seppä HEJ, Keinman HK, Martin GR: Platelet-derived growth factor is a chemoattractant for vascular smooth muscle cells. J Cell Physiol 1982;113:261-266.

Gryglewski RJ, Bunting RM, Vane JR: Mediators produced by the endothelial cell. Hypertension 1988;12:530-548.

Guyton AC: Textbook of medical physiology (7th edition). WB Saunders Company, Philadelphia, 1986.

Hadrava V, Tremblay J, Hamet P: Abnormalities in growth characteristics of aortic smooth muscle cells in spontaneously hypertensive rats. Hypertension 1989;13:589-597.

Hahn AWA, Resink TJ, Scott-Burdon T, Powell J, Dohi Y, Bühler FR: Stimulation of endothelin mRNA and secretion in rat vascular smooth muscle cells: A novel autocrine function. Cell Regul 1990;1:649-659.

Han C, Li J, Minneman KP: Subtypes of alpha 1-adrenoceptors in rat blood vessels. Eur J Pharmacol 1990;190:97-104.

Hannan RL, Kourembanas S, Flanders KC, Rogelj SJ, Roberts AB, Faller DV, Klagsbun $\mathrm{M}$ : Endothelial cells synthesize basic fibroblast growth factor and transforming growth factor beta. Growth Factors 1988;1:7-17.

Hansson GK, Jonasson L, Seifert PS, Stemme S: Immune mechanisms in atherosclerosis. Arteriosclerosis 1989;9:567-578.

Harder DR: Pressure-dependent membrane depolarization in cat middle cerebral artery. Circ Res 1984;55:197-202. 
Harder DR, Sanchez-Ferrer C, Kauser K, Stekiel WJ, Rubanyi GM: Pressure releases a transferable endothelial contractile factor in cat cerebral artery. Circ Res 1989;65:193198.

Harlan JM, Thompson PJ, Bowen-Hope DF: Alpha-thrombin induces release of plateletderived growth factor-like molecule(s) by human endothelial cells. J Cell Biol 1986;103:1129-1133.

Head RJ: Hypernoradrenergic innervation and vascular smooth muscle hyperplastic change. Blood Vessels 1991;28:173-178.

Heagerty AM, Bund SJ, Aalkjaer C: Effects of drug treatment on human resistance arteriole morphology in essential hypertension. Direct evidence for structural remodeling of resistance vessels. The Lancet 1988;2:1209-1212

Heesen B-J, De Mey JGR: Effects of cyclic AMP-affecting agents on contractile reactivity of isolated mesenteric and renal resistance arteries of the rat. $\mathrm{Br} \mathrm{J}$ Pharmacol 1990; $101: 859-864$.

Henrion D, Laher I, Laporte R, Bevan JA: ATII amplifies arterial contractile response to norepinephrine without increasing $\mathrm{Ca}^{2+}$ influx: role of protein kinase $\mathrm{C}$. J Parmacol Exp Ther 1992;261:835-840.

Hermann LM, Castellot JJ: Regulation of vascular smooth muscle cell growth by endothelialsynthesized extracellular matrices. Arteriosclerosis 1987;7:463-469.

Hermsmeyer $\mathrm{K}$ : Altered arterial muscle ion transport mechanisms in the spontaneously hypertensive rat. J Cardiovasc Pharmacol 1984;6 (suppl 1):10-15.

Hermsmeyer $\mathbf{K}$ Harder D: Membrane ATP-ase mechanisms of $\mathrm{K}^{+}$-retum relaxation in arterial muscles of stroke-prone SHR and WKY. Am J Physiol 1986;250:C557-C562.

Hirata Y, Takagi Y, Fukuda Y, Marumo F: Endothelin is a potent mitogen for rat vascular smooth muscle cells. Atherosclerosis 1989;78:225-228.

Houston DS, Shepherd JT, Vanhoutte PM. Adenosine nucleotides, serotonin and endothelium-dependent relaxations to platelets. Am J Physiol 1985;248:H389-H395.

Hsieh H-J, Li N-Q, Frangos JA: Shear stress increases endothelial platelet-derived growth factor mRNA levels. Am J Physiol 1991;260:H642-H646.

Hume WR: Proline and thymidine uptake in rabbit ear artery segments in vitro increased by chronic tangential load. Hypertension 1980;2:738-743.

Hutchins PM, Darnell AE: Observations of a decreased number of small arterioles in spontaneously hypertensive rats. Circ Res 1974;34 (suppl 1):161-165.

Hüttner I, Gabbiani G: Vascular endothelium in hypertension, In: Genest $\mathrm{J}$, Kuchel $\mathrm{O}$, Hamet P, Cantin M (eds): Hypertension, New York, McGraw-Hill, 1983, pp473-488.

Ihara M, Noguchi K, Saeki T, Fukuroda T, Tsuchida S, Kimura S, Fukami T, Ishikawa K, Nishikibe $M$, Yano M: Biological profiles of highly potent novel endothelin antagonists selective for the ET, receptor. Life Sci 1992;50:247-255.

Inagami T, Iwai N, Sasaki K, Yamamo Y, Bardhan S, Chaki S, Guo D-F, Furuta H: Cloning. expression and regulation of ATII receptors. J Hypertension 1992;713-716.

Isles CG, Walker LM, Beevers GD et al: Mortality in patients of the Glasgow Blood Pressure Clinic. J Hypertension 1986;4:141-156.

Itoh TT, Ogawa K, Enomoto J, Hashimoto $\mathrm{H}$, Kai J, Satake K: Comparison of effects of $\mathrm{PGI}_{2}$ and $\mathrm{PGE}_{1}$ on coronary and systemic hemodynamics and on coronary arterial cyclic nucleotide levels in dogs. Adv Prostagl Thromb Leukotr Res 1980; 7:641-646.

Izzo JL, Smith RJ, Larrabee PS, Kallay MC: Plasma norepinephrine and age as determinants of systemic hemodynamics in men with essential hypertension. Hypertension 1987;9:415-419. 


\section{REFERENCES}

Jackson CL, Schwartz SM: Pharmacology of smooth muscle cell replication. Hypertension 1992;20:713-736.

Jawien A, Bowen-Pope DF, Lindner V, Schwartz SM, Clowes AW: Platelet-derived growth factor promotes smooth muscle migration and intimal thickening in a rat model of balloon angioplasty. J Clin Invest 1992;89:507-511.

Jespersen LT, Nyborg NCB, Pedersen OL, Mikkelsen EO, Mulvany MJ: Cardiac mass and peripheral vascular structure in hydralazine-treated spontaneously hypertensive rats. Hypertension 1985; 7:734-741.

Jonasson L, Holm, J, Skalli O, Bondjers G, Hansson GK: Regional accumulations of T-cells, macrophages, and smooth muscle cells in the human atherosclerotic plaque. Arteriosclerosis 1986;6:131-138.

Joseph-Silverstein J, Rifkin DB: Endothelial cell growth factors and the vessel wall. Seminars in thrombosis and hemostasis. 1987;13(4):504-513.

Joshua IG, Wiegman DL, Harris PD, Miller FN: Progressive microvascular alterations with the development of renovascular hypertension. Hypertension 1984;6:61-67.

Judy WV, Watanabe AM, Henry DP, Besch HR, Murphy WR, Hockel GM: Sympathetic nerve activity. Role in regulation of blood pressure in the spontaneously hypertensive rat. Circ Res 1976;38 (suppl II):21-29.

Julius S, Hansson L: Classification of hypertension. In: Hypertension, physiopathology and treatment (eds: Genest J, Kuchel O, Hamet P, Cantin M). McGraw-Hill Book Company, New York, 1983:679-682.

Kahn AM, Cragoe jr. J, Allen C, Halligan RD, Shelat $\mathrm{H}: \mathrm{Na}^{+}-\mathrm{H}^{+}$and $\mathrm{Na}^{+}-$dependent $\mathrm{Cl}^{-}$ $\mathrm{HCO}_{3}^{-}$exchange control $\mathrm{pH}_{\mathrm{i}}$ in vascular smooth muscle. Am J Physiol 1990;259:C134C143.

Kalsner S: Cholinergic mechanisms in human coronary artery preparations: implications of species differences. J Physiol 1985;358:509-516.

Kanbe T, Nara Y, Tagami M, Yamori $T$ : Studies of hypertension-induced vascular hypertrophy in cultured smooth muscle cells from spontaneously hypertensive rats. Hypertension 1983;5:887-892.

Katusic ZS, Shepherd JT, Vanhoutte PM: Vasopressin causes endothelium-dependent relaxations of canine basilar artery. Circ Res 1984;55:575-579.

Katusic ZS, Shepherd JT, Vanhoutte PM: Anoxic contractions in isolated cerebral arteries. Contributions of endothelium-derived factors, metabolites of arachidonic acid and calcium entry. J Cardiovasc Pharmacol 1986;8(suppl 8);97-101.

Katusic ZS, Shepherd JT, Vanhoutte PM: Endothelium-dependent contractions to stretch in canine basilar arteries. Am J Physiol 1987;252:H671-H673.

Kawahara Y, Sunako M, Tsuda T, Fukuzaki H, Fukumoto Y, Takai Y: Angiotensin II induces expression of the $\mathrm{c}$-fos gene through protein kinase $\mathrm{C}$ activation and calcium mobilization in cultured vascular smooth muscle cells. Biochem Biophys Res Commun 1988;150:52-59.

Kifor I, Dzau VJ: Endohelial renin-angiotensin pathway: Evidence for intracellular synthesis and secretion of angiotensin. Circ Res 1987;60:422-428.

Kim SJ, Angel P, Lafyatis R, Hattri K, Young Kim K, Sporn MB, Karin M, Roberts AB: Autoinduction of transforming growth factor $B_{1}$ is mediated by the AP-1 complex. Mol Cell Biol 1990;10:1492-1497.

Kishi K, Inoue T: Possible mechanisms of abnormal norepinephrine sensitivity and reactivity of resistance vessels and the development of hypertension in spontaneously hypertensive rats. Am J Hypertension 1990;3:202S-205S. 
KJagsbrun M, Fdelman ER: Biological and biochemical properties of fibroblast growth factors: implications for the pathogenesis of atherosclerosis. Atherosclerosis 1989;9:269-278.

Komuro I, Kurihara H, Sugiyama T, Takaku F, Yazaki Y: Endothelin stimulates c-fos and c-myc expression and proliferation of vascular smooth muscle cells. FEBS Lett 1988:238:249-252.

Konishi M, Su C: Role of the endothelium in dilator responses of spontaneously hypertensive rat arteries. Hypertension 1983;5:881-886.

Koo EWY, Gotlieb AI: Endothelial stimulation of intimal cell proliferation in porcine aortic organ culture. Am J Pathol 1989;134:497-503.

Kosgaard N, Mulvany MJ: Cellular hypertrophy in mesenteric resistance vessels from renal hypertensive rats. Hypertension 1988; 12:162-167.

Koutousov S, Remmal A, Marche P, Meyer P: Hypersensitivity of phospholipase-C in platelets of spontaneously hypertensive rats. Hypertension 1987;10:497-504.

Kurihara H, Yoshizumi M, Sugiyama T, Takaku F, Yanagisawa M, Masaki T: Transforming growth factor- $B$ stimulates the expression of endothelin mRNA by vascular endothelial cells. Biochem Biophys Res Commun;159(3):1435-1440.

Kuriyama S, Nakamura A, Hopp L, Fine BP, Kino M, Cragoe E, Aviv A: ATII effect on na-transport in vascular smooth muscle cell. J Cardiovasc Pharmacol 19988;11:139146.

Labarca C, Paigen K: A simple and sensitive DNA assay procedure. Anal Biochem 1980;102:344-352.

Lais LT, Brody MJ: Vasoconstrictor hyperresponsiveness: an early pathogenic mechanism in the spontaneously hypertensive rat. Eur J Pharmacol 1978;47:177-189.

Langille BL, Reidy MA, Kline RJ. Injury and repair of endothelium at sites of flow disturbances near abdominal aortic coarctations in rabbits. Arteriosclerosis 1986;6:146154.

Langille BL, O'Donnel: Reductions in arterial diameter produced by chronic decrease in blood flow are endothelium dependent. Science 1986;231:405-407.

Langille BL, Bendeck MP, Keeley FW: Adaptations of carotid arteries of young and mature rabbits to reduce carotid blood flow. Am J Physiol 1989;256:H931-H939.

Lappe RW, Brody MJ: Mechanisms of central pressor action of ATII in conscious rats. Am J Physiol 1984;246:R56-R62.

Laurenzi M, Mancini M, Menotti A, Stamler J, Trevisan M, Zanchetti A: On behalf of the Gubbio Study Group: Multiple risk factors in hypertension: results from the Gubbio Study. J.Hypertension 1990;8(suppl. 1):7-12.

Lee RMKW, Smeda JS: Primary versus secondary structural changes of the blood vessels in hypertension. Can J Physiol Pharmacol 1985;63:392-401.

Lee RMKW: Vascular changes at the prehypertensive phase in the mesenteric arteries from spontaneously hypertensive rats. Blood Vessels 1985;22:105-126.

Lee SA, Hurwitz J: Mechanism of elongation of primed DNA by DNA polymerase delta, proliferating cell nuclear antigen, and activator 1. Proc Acad Sci USA 1990;87:58725876.

Lee SL, Wang WW, Moore BJ, Fanburg BL: Dual effect of serotonin on growth of bovine pulmonary artery smooth muscle cells in culture. Circ Res 1991;68:1362-1368.

Le Noble JLML, Smith TL, Hutchins PM, Struijker Boudier HAJ: Microvascular alterations in adult conscious spontaneously hypertensive rats. Hypertension 1990;15: 415-420.

Lever AF: Slow pressor mechanisms in hypertension: a role for hypertrophy of resistance 


\section{REFERENCES}

vessels? J Hypertension 1986;4:515-524.

Lever AF, Harrap SB: Essential hypertension: a disorder of growth with origins in childhood? J Hypertension 1992; 10:101-120.

Levi BI, Michel JB, Salzmann JZ, Azizi M, Poitevin P, Safar M, Camilleu JP: Effects of chronic inhibition of converting enzyme on mechanical and structural properties of arteries in rat renovascular hypertension. Circ Res 1988;63:344-348.

Libby P, O'Brien KV: Culture of quiescent arterial smooth muscle cells in a defined serumfree medium. J Cell Physiol 1983;115:217-223.

Libby P, Ordovas JM, Birinyi LK, Auger KR, Dinarello CA: Inducible interleukin-1 expression in human vascular smooth muscle cells. J Clin Invest 1986;78:1432-1438.

Libby P, Wamer SJC, Friedman GR: Interleukin 1: A mitogen for human vascular smooth muscle cells that induces the release of growth-inhibitory prostanoids. J Clin Invest 1988;81:487-498.

Libby P, Hansson GK: Biology of disease: involvement of the immune system in human atherogenesis: current knowledge and unanswered questions. Lab Invest 1991;64:5-15.

Lichtenstein A, Brecher P, Chobanian A: Effects of hypertension and its reversal on size and DNA content of rat aortic smooth muscle cells. Hypertension 1986;8:1150-1154.

Lilly LS, Pratt RE Alexander RW, LarsonDM, Ellison KE, Gimbrone MA, Dzau VJ: Renin expression by vascular endothelial cells in culture. Circ Res 1985;57:312-318.

Limas C, Westrum B, Iwah J, et al: Aortic morphology in salt-dependent genetic hypertension. Am J Pathol 1982;107:378-394.

Lindholm L, Ejlertsson G, Scherstén B: High risk of cerebrovascular morbidity in well treated male hypertensives. Acta Med Scand 1984;216:251-259.

Lockette WE, Otsuha Y, Carretero OA: Endothelium-dependent relaxation in hypertension. Hypertension 1986;8 (suppl 2):61-66.

Loeb AL, Bean BL: Antihypertensive drugs inhibit hypertension-associated aortic DNA synthesis in the rat. Hypertension 1986;8:1135-1142.

Loeb AL, Mandel HG, Straw JA, Bean BL: Increased aortic DNA synthesis precedes renal hypertension in rats: an obligatory step. Hypertension Dallas 1986;8:754-761.

Loppnow H, Libby P: Proliferating or interleukin-1 activated human vascular smooth muscle cells secrete copious interleukin-6. J Clin Invest 1990;85:731-738.

Lundin SA, Hallback-Nordlander MIL: Regression of structural cardiovascular changes by antihypertensive therapy in spontaneously hypertensive rats. J Hypertension 1984;2:1118.

Lundin S, Ricksten SE, Thoren: Renal sympathetic activity in spontaneously hypertensive rats and normotensive controles, as studied by three different methods. Acta Physiologica Scand 1984;120:265-272.

Lund-Johansen P: Haemodynamics in essential hypertension. Clin Sci 1980;59:343-354.

Lund-Johansen P: Hemodynamics in essential hypertension: still an area of controversy. J Hypertension 1983;1:209-213.

Lungier C, Schini VB. Characterization of cyclic nucleotide phosphodiesterases from cultured bovine aortic endothelial cells. Biochem Pharmacol 1990;39:75-84.

Lüscher TF, Vanhoutte PM: Endothelium-dependent contractions to acetylcholine in the aorta of the spontaneously hypertensive rat. Hypertension 1986;8:344-348.

Lüscher TF, Romero JC, Vanhoutte PM: Bioassay of endothelium-derived vasoactive substances in the aorta of normotensive and spontaneously hypertensive rats. J Hypertension 1986;4 (suppl 6):81-83.

Lüscher TF, Raij L, Vanhoutte PM: Endothelium-dependent responses in normotensive and 
hypertensive Dahl rats. Hypertension 1987;9:157-163.

Lüscher TF: Endothelial vasoactive substances and cardiovascular disease. Karger, Basel, 1988.

Lüscher TF. Imbalance of endothelium-derived relaxing and contracting factors. A new concept in hypertension? Am J Hypertension 1990;3:317-330.

Lüscher TF, Boulanger CM, Dohi Y, Yang Z: Endothelium-derived contracting factors. Hypertension 1992;19:117-130.

Luscinskas FW, Cybulski MI, Keily JM, Perkins CS, Davis VM, Gimbrone MA. Cytokineactivated human endothelial monolayers support neutrophil an transmigration via mechanisms involving both endothelial leucocyte adhesion molecules and intracellular adhesion molecules. J Immunol 146: 1617-1625, 1991

MacMahon SW, Cutler JA, Furberg CD: The effects of drug treatment for hypertension on morbidity and mortality from cardiovascular disease: a review of randomized controlled trials. Prog Cardiovasc Dis 1986;29 (suppl 1):99-118.

MacMahon S, Peto R, Cutler J etal: Blood pressure, stroke and coronary artery disease.I. Prolonged differences in blood pressure: prospective observational studies corrected for the regression dilution basis. Lancet 1990;335:765-774.

Madshus IH: Regulation of intracellular pH in eukaryotic cells. Biochem J 1988;250:1-8.

Majack RA, Bomstein P: Heparin and related glycosaminoglycans modulate the secretory phenotype of vascular smooth muscle cells. J Cell Biol 1984;99:1688-1695.

Majack RA, Cook SC, Bornstein P: Platelet-derived growth factor and heparin-like glycosamineglycans regulate thrombospondin synthesis and deposition in the matrix of smooth muscle cells. J Cell Biol 1985;101:1059-1070.

Majack RA, Cook SC, Bomstein P: Control of smooth muscle cell growth by components of the extracellular matrix: Autocrine role for thrombospondin. Proc Natl Acad Sci USA 1986;83:9050-9054.

Majack RA: Beta-type transforming growth factor specifies organizational behavior in vascular smooth muscle cell cultures. J Cell Biol 1987;105:465-471.

Majack RA, Majesky MW, Goodman LV: Role of PDGF-A expression in the control of vascular smooth muscle cell growth by transforming growth factor- $B$. J Cell Biol 1990;111:239-247.

Majesky MW, Schwartz SM, Clowes MM, Clowes AW: Heparin regulates smooth muscle $S$ phase entry in the injured rat carotid artery. Circ Res 1987;61:296-300.

Majesky MW, Benditt EP, Schwartz SM: Expression and developmental control of plateletderived growth factor A-chain and B-chain/cis genes in rat aortic smooth muscle cells. Proc Natl Acad Sci USA 1988;85:1524-1528.

Majesky MW. Daemen MJAP, Schwartz SM: $\alpha_{1}$-Adrenergic stimulation of platelet-derived growth factor A-chain gene expression in rat aorta. J Biol Chem 1990';265:1082-1088.

Majesky MW, Reidy MA, Bowen-Pope DF, Hart CE, Wilcox JN, Schwartz SM: PDGF ligand and receptor gene expression during repair of arterial injury. J Cell Biol $1990^{\mathrm{b}} ; 111: 2149-2158$.

Mantovani A, Dajena E: Cytokines as communication signals between leukocytes and endothelial cells. Immun Today 1989;10(11):370-375.

Martin M, Resch K. Interleukin I: more than a mediator between leukocytes. TIPS 1989;9:171-177.

Martinez-Maldonado $\mathrm{M}$ : Pathophysiology of renovascular hypertension. Hypertension 1991;17:707-719.

Mauger JP, Worcel M, Tassin J, Courtois Y: Contractility of smooth muscle cells of rabbit 
aorta in tissue culture. Nature 1975;255:337-338.

McGuire PG, Twietmeyer TA: Aortic endothelial junctions in developing hypertension. Hypertension 1985;7:483-490.

McLean IW, Nakoine PK: Periodate-lysine-paraformaldehyde fixative: A new fixative for immunoelectron microscopy. J Histochem Cytochem 1974;22:1077-1082.

Merrilees M, Scott L: Organ culture of rat carotid artery: Maintenance of morphological characteristics and pattern of matrix synthesis. In Vitro 1982;18:900-910.

Michel MC, Kanczik R, Kamssi M, Knorr A, Siegl H, Beckeringh JJ, Brodde OE: $\alpha$ - and $B$-adrenoceptors in hypertension. I. Cardiac and renal $\alpha_{1}-\beta_{1}$ and $\beta_{2}$-adrenoceptors in rat models of acquired hypertension. J Cardiovasc Pharmacol 1989;13:421-431.

Michel MC, Brodde OE, Insel PA: Peripheral adrenergic receptors in hypertension. Hypertension 1990;16:107-120.

Miller VM, Vanhoutte PM: Endothelium-dependent contractions to arachidonic acid are mediated by products of cyclooxygenase. Am J Physiol 1988;248:H432-H437.

Mohrman DE: Local metabolic influences on resistance vessels. in: The Resistance Vasculature. Bevan, Humana, Totowa, New Jersey, USA, 1991, 241-250.

Moncada S, Vane JR: Pharmacology and endogenous roles of prostaglandin endoperoxides, thromboxane $A_{2}$ and prostacyclin. Pharmacol Rev 1979;30:293-331.

Moncada S, Radomski MW, Palmer RMJ: Endothelium-derived relaxing factor: identification as nitric oxide and role in the control of vascular tone and platelet function. Biochem Pharmacol 1988;37:2495-2501.

Moncada S, Higgs EA: Endogenous nitric oxide: physiology, pathology and clinical relevance. Eur J Clin Invest 1991;21:361-374.

Morasaki N, Kanzaki T, Motoyama N, Saito Y, Yoshida S: Cell cycle-dependent inhibition of DNA synthesis by prostaglandin $\mathrm{I}_{2}$ in cultured rabbit aortic smooth muscle cells. Atherosclerosis 1988;71:165-171.

Moss PRL, Campbell GR, Wang ZL, Campbell JH: Smooth muscle phenotypic expression in human carotid arteries: 1. Comparison of cel]s from diffuse intimal thickening adjacent to those of therioma plaques with those of the media. Lab Invest 1985;53:556562 .

Mullins M, Peters J, Ganten D: Fulminant hypertension in transgenic rats harbouring the mouse Ren-2 gene. Nature 1990;344:541-544.

Mulvany MJ, Halpern W: Contractile properties of small arterial resistance vessels in spontaneously hypertensive and normotensive rats. Circ Res 1977;41:19-26.

Mulvany MJ, Hanssen PK, Aalkjaer C: Direct evidence that the greater contractility of resistance vessels in spontaneously hypertensive rats is associated with a narrowed lumen, a thicker media, and a greater number of smooth muscle cell layers. Circ Res 1978;43:854-864.

Mulvany MJ, Nyborg NCB: An increased calcium sensitivity of mesenteric resistance vessels in young and adult spontaneously hypertensive rats. Br J Pharmacol 1980;71: 585-596.

Mulvany MJ, Baandrup U, Gundersen HJG: Evidence for hyperplasia in mesenteric resistance vessels of spontaneously hypertensive rats using a three-dimensional dissector. Circ Res 1985;57:794-800.

Mulvany MJ: Role of vascular structure in blood pressure development of the spontaneously hypertensive rat. J Hypertension 1986;4 (suppl 3):61-63.

Munro JM, Cotran RS: Biology of disease. The pathogenesis of atherosclerosis: Atherogenesis and inflammation. Lab Invest 1988;58(3):249-261.

Mulvany MJ: Changes to the vascular system resulting from hypertension and their effects 


\section{REFERENCES}

on response to therapy. Drugs 1990;39 (suppl 1):9-20.

Nabata T, Morimoto S, Koh E, Shiraishi T, Ogihara T: Interleukin-6 stimulates c-myc expression and proliferation of cultured vascular smooth muscle cells. Biochem Int 1990;20:445-453.

Naftilan AJ, Pratt RE, Dzau VJ: Induction of platelet-derived growth factor A-chain and cmyc gene expression by angiotensin II in cultured rat vascular smooth muscle cells. J Clin Invest 1989;83:1419-1424.

Nakaki T, Nakayama $M$, Yamamoto $S$, Kato R: $\alpha_{1}$-adrenergic stimulation and $B_{2}$-adrenergic inhibition of DNA synthesis in vascular smooth muscle cells. Mol Pharmacol 1990;37:30-36.

Nemecek GM, Coughlin SR, Handley DA, Moskowitz MA: Stimulation of aortic smooth muscle cell mitogenesis by serotonin. Proc Natl Acad Sci USA 1986;83:674-678.

$\mathrm{Ng}$ KKF, Vane JR: Conversion of angiotensin I to angiotensin II. Nature 1967;216:762-766.

Nilson C-H, Westermark B, Thyberg J: Demonstration of stimulatory effects of plateletderived growth factor on cultivated rat arterial smooth muscle cells: differences between cells from young and adult animals. Exp Cell Res 1983;145:231-237.

Noden D: Embryonic origins and assembly of blood vessels. Am Rev Respir Dis 1989;140:1097-1103.

Nyborg NCB, Mulvany MJ: Lack of effect of antihypertensive treatment with felodipine on cardiovascular structure of young spontaneously hypertensive rats. J Cardiovasc Res $1985 ; 19: 528-536$.

Nyborg NCB, Bevan JA: Increased alpha-adrenergic receptor affinity in resistance vessels from hypertensive rats. Hypertension 1988;11:635-638.

Okamoto K, Aoki K: Development of a strain of spontaneously hypertensive rats. Jpn Circ J 1963;27:282-293.

Olson NE, Chao S, Lindner V, Reidy MA: Intimal smooth muscle cell proliferation after balloon catheter injury. The role of basic fibroblast growth factor. Am J Pathol 1992;140(5): 1017-1023.

Owens GK, Schwartz SM: Alteration in vascular smooth muscle mass in the spontaneously hypertensive rat. Role of cellular hypertrophy, hyperploidy, and hyperplasia. Circ Res 1982;51:280-289.

Owens GK, Schwartz SM: Vascular smooth muscle cell hypertrophy and hyperploidy in the Goldblatt hypertensive rat. Circ Res 1983;53:491-501.

Owens GK: Differential effects of antihypertensive drug therapy on vascular smooth muscle cell hypertrophy, hyperploidy, and hyperplasia in the spontaneously hypertensive rat. Circ Res 1985;56:525-536.

Owens MP, Bevan JA: Acetylcholine-induced endothelial-dependent vasodilatation increased as artery diameter decreases in the rabbit ear artery. Experientia 1985;41:1057-1058.

Owens G, Reidy M: Hyperplastic growth responses of vascular smooth muscle cells following induction of acute hypertension in rats by aorta coarctation. Circ Res 1985;57:695-705.

Owens GK: Influence of blood pressure on development of aortic medial smooth muscle hypertrophy in spontaneously hypertensive rats. Hypertension 1987;9:178-187.

Owens GK, Schwartz SM, McCanna M: Evaluation of medial hypertrophy in resistance vessels of spontaneously hypertensive rats. Hypertension 1988; $11: 198-207$.

Owens GK, Geisterfer AAT, Wei-Hwa Yang Y, Komoriya A: Transforming growth factor beta induced growth inhibition and cellular hypertrophy in cultured vascular smooth muscle cell. J Cell Biol 1988' $771-780$. 


\section{REFERENCES}

Owens GK: Control of hypertrophic versus hyperplastic growth of vascular smooth muscle cells. Am J Physiol 1989;257:H1755-H1765.

Pang SC: In vitro proliferation of aortic smooth muscle cells from spontaneously hypertensive and normotensive rats. J Pathology 1989;158:167-173.

Palmer RMJ, Ferrigo AG, Moncada S: Nitric oxide release accounts for the biological activity of endothelium-derived relaxing factor. Nature 1987;327:524-525.

Paquet JL, Baudouin-Legros M, Marche P, Meyer P: Enhanced proliferating activity of cultured smooth muscle cells from SHR. Am J Hypertension 1989;2:108-110.

Pegg AE, McCann PP: Polyamine metabolism and function. Am J Physiol 1982;243:C.212C221.

Penttinen RT, Kobayashi S, Bornstein P: Transforming growth factor B increases mRNA for matrix proteins both in the presence and in the absence of changes in mRNA stability. Proc Natl Acad Sci USA 1988;85:1105-1108.

Phillips MI: Functions of angiotensin in the central nervous system. Ann Rev Physiol 1987;49:413-435.

Pober JS, Collins T, Gimbrone MA, Libby P, Reiss CS: Inducible expression of class II major histocompatability complex antigens and the immunogenicity of vascular endothelium. Transplantation 1986;41:141-146.

Pohl U, Holtz J, Busse R et al: Crucial role of the endothelium in the vasodilator response to increased flow in vivo. Hypertension 1986;8:37-44.

Postov YV: An approach to the explanation of cell membrane alterations in primary hypertension. Hypertension 1990;15(3):332-337.

Powell JS, Clozel JP, Müller RKM, Kuhn H, Hefti F, Hosang M, Baumgartner HR: Inhibitors of angiotensin-converting enzyme prevent myointimal proliferation after vascular injury. Science 1989;245:186-188.

Prewitt RL, Chen IIH, Dowell R: Development of microvascular rarefaction in the spontaneously hypertensive rat. Am J Physiol 1982;243:H243-H251.

Radomski MW, Palmer RMJ, Moncada S: The role of nitric oxide and cGMP in platelet adhesion to vascular endothelium. Biochem Biophys Res Commun 1987^;148:14821489.

Radomski MW, Palmer RMJ, Moncada S: The anti-aggregating properties of vascular endothelium: interaction between prostacyclin and nitric oxide. Br J Pharmacol $1987^{\mathrm{b}} ; 92: 639-646$.

Raines EW, Dower SK, Ross R: Interleukin-1 mitogenic activity for fibroblasts and smooth muscle cells is due to PDGF-AA. Science 1989;243:393-396.

Ramaekers FCS, Puts JJG, Moesker O, Kant A, Huysmans A, Haag D, Jap PHK, Herman CJ, Vooijs GP: Antibodies to intermediate filament proteins in the immunoistochemical identification of human tumors: an overview. Histochem J 1983;15:691-713.

Rao GN, Sardet C, Pouyssegur J, Berk BC: Differential regulation of $\mathrm{Na}^{+} / \mathrm{H}^{+}$antiporter gene expression in vascular smooth muscle cells by hypertrophic and hyperplastic stimuli. J Biol Chem 1990;265(32): 19393-19396.

Rappoport RM, Murad F. Endothelium-dependent and nitrovasodilator-induced relaxation of vascular smooth muscle: role of cyclic GMP. J Cyclic Nucleo Protein Phosphor Res 1983;9:281-296.

Raymond JR, Hnatowich M, Lefkowitz RJ, Caron MG: Adrenergic receptors: models for regulation of signal transduction. Hypertension 1990;15:119-131.

Re RN, Labiche RA, Bryan SE: Nuclear-hormone mediated changes in chromatin solubility. Biochem Biophys Res Commun 1983;110:61-68. 


\section{REFERENCES}

Reidy MA, Silver M: Endothelial regeneration VII. Lack of intimal proliferation after defined injury to rat aorta. Am J Pathol 1985;118:173-178.

Reidy MA: Proliferation of smooth muscle cells at sites distant from vascular injury. Atherosclerosis 1990;10:298-305.

Reilly CF, Fritze LM, Rosenberg RD: Antiproliferative effects of heparin on vascular smooth muscle cells are reversed by epidermal growth factor. J Cell Physiol $1987 ; 131: 149-157$.

Resink TJ, Scott-Burdon T, Baur U, Bühler FR: Increased proliferation and phosphoinositide tumover in cultured smooth muscle cell from spontaneously hypertensive rats. J Hypertension 19875 (suppl 5):145-148.

Resink TJ, Scott-Burden T, Baur U, Bürgin M, Bühler FR: Decreased susceptibility of cultured smooth muscle cells from SHR rats to growth inhibition by heparin. J Cell Physiol 1989; 138:137-144.

Resink TJ, Scott-Burdon T, Baur U, Burgin M, Bühler FR: Enhanced responsiveness to ATII in vascular smooth muscle cells from spontaneously hypertensive rats is not associated wit alterations in protein kinase C. Hypertension 1989 ; 14:293-303.

Resink TJ, Hahn AWA, Scott-Burden T, Powell J, Webber E, Bühler FR: Inducible endothelin mRNA expression and peptide secretion in cultured human vascular smooth muscle cells. Biochem Biophys Res Commun 1990;168:1303-1310.

Rinaldi G, Bohr D: Endothelium-mediated spontaneous response in aortic rings of deoxycorticosterone acetate-hypertensive rats. Hypertension 1989;13:256-261.

Rosen EM, Goldberg ID, Shapiro HM, Levenson SE, Halpin PA, Faraggi D: Strain and site dependence of polyploidization of cultured rat smooth muscle. J Cell Physiol 1986; 128:337-344.

Ross R, Glomset J, Kariya B, Harker L: A platelet-dependent serum factor that stimulates the proliferation of arterial smooth muscle cells in vitro. Proc Natl Acad USA 1974;71:1207-1210.

Ross R, Glomset J: The pathogenesis of atherosclerosis. New Engl J Med 1976; 295: 369377 and $420-425$.

Ross R, Nist C, Kariya B, Rivest MJ, Raines E, Callis J: Physiological quiescence in plasma-derived serum: influence of platelet-derived growth factor on cell growth in culture. J Cell Physiol 1978;97:497-508.

Ross R: Atherosclerosis: A problem of arterial wall cells and their interaction with blood components. Atherosclerosis 1981;1:293-311.

Ross R, Glomset JA: Atherosclerosis and the arterial smooth muscle cell. Science $1985 ; 180: 1332-1339$.

Ross R: The pathogenesis of atherosclerosis: an update. New Engl J Med 1986;314:488-450.

Ross R, Raines EW, Bowen-Pope DF: The biology of platelet-derived growth factor. Cell 1986;46: 155-169.

Rubanyi GM, Vanhoutte PM. Hypoxia releases a vasoconstrictor substance from canine vascular endothelium. J Physiol 1985;364:45-56.

Rubanyi GM, Romeero JC, Vanhoutte PM: Flow-induced release of endothelium-derived relaxing factor. Am J Physiol 1986;250:H1145-H1149.

Ruffolo RR Nichols AJ, Stadel JM, Hieble JP: Structure and function of alpha-adrenoceptors. Pharmacol Rev 1991;43:475-505.

Safar ME: Therapeutic trials and large arteries in hypertension. Am Heart J 1988;115:702 710.

Sahlgren B, Eklof AC, Aperia A: Studies on the renal component of the hypertension in rats 


\section{REFERENCES}

with aortic coarctation. Role of angiotensin II. Acta Physiol Scand 1986;127:443-448.

Saijonmaa O, Ristimäki A, Fyhrquist F: Atrial natriuretic peptide, nitroglycerine, and nitroprusside reduce basal and stimulated endothelin production from cultured endothelial cells. Biochem Biophys Res Commun 1990;173:514-520.

Sano T, Tarazi RC. Differential structural responses of small resistance vessels to antihypertensive therapy. Circulation 75: 618-626, 1987

Saye JA, Singer HA, Peach MJ: Role of the endothelium in conversion of angiotensin I to angiotensin II in rabbit aorta. Hypertension 1984;6:216-221.

Schelling P, Fischer H, Ganten D: Angiotensin and cell growth: a link to cardiovascular hypertrophy? J Hypertension 1991;9:3-15.

Schiffers PMH, Fazzi GE, Van Ingen Schenau D, De Mey JGR: Effects of candidate autocrine and paracrine mediators on growth responses in isolated arteries of the rat (Arteriosclerosis and Thrombosis, accepted).

Schlessinger J, Schreiber AB, Levi A, Lax I, Libermann T, Yarden Y: Regulation of cell proliferation by epidermal growth factor. Crit Rev Biochem 1983;14:93-111.

Schwartz S, Ross R: Cellular proliferation in atherosclerosis and hypertension. Prog Cardiovasc Dis 1984;26:355-372.

Schwartz SM: Smooth muscle proliferation in hypertension. Hypertension 1984;6 (suppl I):56-61.

Schwartz SM, Campbell GR, Campbell JH. Replication of smooth muscle cells in vascular disease. Circ Res 1986;58:427-444.

Schwartz SM, Reidy MA: Common mechanisms of proliferation of smooth muscle in atherosclerosis and hypertension. Hum Pathol 1987;18:240-247.

Scott TM, Galway G: The relationship between altered blood vessel structure, hypertension and the sympathetic nervous system. Can J Physiol Pharmacol 1985;63:387-391.

Scott-Burden T, Bühler FR: Regulation of smooth muscle proliferative phenotype by heparinoid-matrix interactions. TIPS 1988;9:94-98.

Scott-Burden T, Resink TJ, Baur U, Bürgin M, Bühler FR: Amiloride sensitive activation of S6-kinase by angiotensin II in cultured vascular smooth muscle cells. Biochem Biophys Res Commmun 1988;151:583-589.

Scott-Burden T, Resink TJ, Bühler FR: Enhanced growth and growth factor responsiveness of vascular smooth muscle cells from hypertensive rats. J Cardiovasc Pharmacol 1989:;14 (suppl 6):16-21.

Scott-Burden T, Resink TJ, Baur U, Bürgin M, Bühler: Epidermal growth factor responsiveness in smooth muscle cells from hypertensive and normotensive rats. Hypertension $1989^{b} ; 13: 295-304$.

Scott-Burden T, Resink TJ, Hahn AWA, Bühler FR: Angiotensin II induction of growth related metabolism in cultured vascular smooth muscle cells. Am I Hypertension 1991;4:183-188.

Seidel CL, Allen JC, Wallace CL, Jemelka SK, Navran SS, Denusion DK: Relation between cell $\mathrm{Na}$, Na-pump number myosin expression and proliferation in cultured canine vascular smooth muscle celis. In: Molecular biology of the cardiovascular system, pp 269-276, Alan R. Liss Inc., 1990.

Severs WB, Daniels-Severs AE: Effects of angiotensin on the central nervous system. Pharmacol Rev 1973;25:415-449.

Shebuski RH, Aiken JW: ATII stimulation of renal prostaglandin synthesis elevates circulating prostacyclin in the dog. J Cardiovasc Parmacol 1980;2:667-677.

Shepherd JT: Increased systemic vascular resistance and primary hypertension: the expanding 
complexity. J Hypertension 1990;8 (suppl 7):15-27.

Shimokade K, Raines WE, Madtes DK, Barret TB, Benditt EP, Ross RA: Significant part of macrophage-derived growth factor consists of at least two forms of PDGF. Cell 1986; 43:277-286.

Shimokawa H, Aarhus LI, Vanhoutte PM: Porcine coronary arteries with regenerated endothelium have a reduced endothelium-dependent responsiveness to aggregating platelets and serotonin. Circ Res 1987;61:256-270.

Sibley DR, Lefkowitz RI: Molecular mechanisms of receptor desensitization using the betaadrenergic receptor-coupled adenylate cyclase system as a model. Nature 1985;317:124-129.

Simonson MS, Dunn MJ: Cellular signalling by peptides of the endothelin gene family. Faseb J 1990;4:2989-3000.

Sivertsson R: The hemodynamic importance of structural vascular changes in essential hypertension. Acta Physiol Scand 1970;343 (suppl):1-56.

Sjölund M, Hedin U, Sejersen T, Heldin C-H, Thyberg J: Arterial smooth muscle cell express platelet-derived growth factor (PDGF) A chain mRNA, secrete a PDGF-like mitogen and bind exogenous PDGF in a phenotype and growth state dependent manner. J Cell Biol 1988;106:403-413.

Skalli O, Ropraz P, Trzeciak A, Benzonana G, Gillesen D, Gabbiani A: A monoclonal antibody against alpha-smooth muscle actin: a new probe for smooth muscle differentiation. J Cell Biol 1986;103:2787-2796.

Smeda JS, Lee RMKW, Forrest JB: Structural and reactivity alterations of the renal vasculature of spontaneously hypertensive rats prior to and during established hypertension. Circ Res $1988^{\mathrm{x}} ; 63: 518-533$.

Smeda JS, Lee RMKW, Forrest JB: Prenatal and postnatal hydralazine treatment does not prevent renal vessel wall thickening. Circ Res 1988 ; $63: 534-542$.

Sokal RR, Rohlf FJ: Biometry. New York: Freeman, 1981, pp 1-859.

Southgate K, Newby AC: Serum-induced proliferation of rabbit aortic smooth muscle cells from the contractile state is inhibited by 8 -Br-cAMP but not 8 -Br-cGMP. Atherosclerosis 1990;82:113-123.

Sporn MB, Robergs AB, Wakefield LM, De Crombrughe B: Some recent advances in the chemistry and biology of transforming growth factor beta. J Cell Biol 1987; 105:10391045 .

Spom MB, Roberts AB: Peptidergic growth factors are multifunctional. Nature 1988;332:217-219.

Staiano-Coico L, Hajjar DP, Hefton JM, Hajjar KA, Kimmel M: Interactions of arterial cells. III. Stathmokinetic analysis of smooth muscle cells cultured with endothelial cells. J Cell Physiol 1988;134:485-490.

Stamberg J, Berkson DM, Lindberg HA: Risk factors: Their role in the etiology and pathogenesis of the atherosclerotic diseases. The pathogenesis of atherosclerosis. Ed Wissler RW, Geer JC, Williams and Wilkins, Baltimore, 1972, 41-119.

Stemerman MB, Ross R: Experimental arteriosclerosis. 1. Fibrous plaque formation in primates: an electron microscopic study. J Exp Med 1973;136:769-789.

Stemme S, Fager G, Hansson GK: MHC class II antigen expression in human vascular smooth muscle cells is induced by interferon-gamma and modulated by tumor necrosis factor and lymphotoxin. Immunology 1990;69:243-249.

Struijker-Boudier HAJ, Van Bortel I,MAB, De Mey JGR: Remodeling of the vascular tree in hypertension: drug effects. TIPS 1990;11:240-245. 
Stuehr DJ, Nathan CF: Nitric oxide: A macrophage product responsible for cytostasis and respiratory inhibition in tumor target cells. J Cell Med 1989;169:1543-1555.

Sugihara H, Hattori T, Fukuda M: Immunohistochemical detection of bromodeoxyuridine in formalin-fixed tissues. Histochemistry 1986;85:193-195.

Terracio L, Ronnstrand L, Tingstrom A, Rubin K, Claesson-Welsh L, Funa K, Heldin CH: Induction of platelet-derived growth factor receptor expression in smooth muscle cells upon tissue culturing. J Cell Biol 1988;107:1947-1957.

Thyberg JL, Palmberg L, Nilsson J, Ksiazek T, Sjölund M: Phenotype modulation in primary cultures of arterial smooth muscle cells: on the role of the platelet-derived growth factor., Differentiation 1983;25:156-167.

Thyberg J, Fredholm BB: Induction of omithine decarboxylase activity and putrescine synthesis in arterial smooth muscle cell stimulated with platelet-derived growth factor. Exp Cell Res 1987;170:160-169.

Thyberg J, Hedin U, Sjölund M, Palmberg L, Bottger BA. Regulation of differentiated properties and proliferation of arterial smooth muscle cells. Arteriosclerosis 1990;10:966-990.

Toda N: Endothelium-dependent relaxations induced by angiotensin II and histamine in isolated arteries of the rat. Br J Pharmacol 1984;81:301-307.

Triggle CR: Reactivity and sensitivity changes of blood vessels in hypertension, in: Blood Vessel Changes in Hypertension: Structure and Function, Lee RMKW, CRC Press Inc., Boca Raton, USA, 1989, 25-44.

Tsukada T, Tippens D, Ross R, Gown A: HHF 35, a muscle actin specific monoclonal antibody. I. Immunocytochemical and biochemical characterization. Am J Pathol $1987 ; 126: 51-60$.

Turla MB, Thompson MM, Corjay MH, Owens GK: Mechanisms of angiotensin-II and arginine vasopressin-induced increases in protein synthesis and content in cultured rat aortic smooth muscle cells. Circ Res 1991;68:288-299.

Uehara Y, Ihii M, Ishimitsu T, Sugimoto T: Enhanced phospholipase-C activity in the vascular wall of SHR. Hypertension 1988;11:28-33.

Vallega GA, Canessa ML, Berk BC, Brock TA, Alexander RW: Vascular smooth muscle $\mathrm{Na}^{+} / \mathrm{H}^{+}$exchanger kinetics and its activation by ATII. Am J Physiol 1988;254:C751C758.

Van Furth R, Van Zwet TL: Immunocytochemical detection of 5-bromo-2-deoxyuridine incorporation in individual cells. J Immunol Methods 1988;108:45-51.

Vanhoutte PM: Heterogeneity in vascular smooth muscle. In Kaley G, Altura BM (eds): Microcirculation. Baltimore University Press, 1978, 2, 181-309.

Vanhoutte PM, Verbeuren TJ, Webb RC: Local modulation of adrenergic neuroeffector interaction in the blood vessel wall. Physiol Rev 1981;61:151-218.

Vanhoutte PM:The end of the quest? Nature 1987;327:459-460.

Vanhoutte PM. The endothelium modulator of vascular smooth muscle tone. New Engl J Med 1988;319:512-513.

Vanhoutte PM: Endothelium and control of vascular function. Hypertension 1989;13:658667.

Van Kleef EM, Smits JF, De Mey JGR, Cleutjens JP, Lombardi DM, Schwartz SM, Daemen MJ: Alpha 1-adrenoceptor blockade reduces the angiotensin Il-induced vascular smooth muscle cell DNA synthesis in the rat thoracic aorta and carotid artery. Circ Res 1992;70:1122-1127.

Van Merode T, van Bortel LM, Smeets FA, Mooij JM, Böhm R, Rahn KH, Reneman RS: 


\section{REFERENCES}

Verapamil and nebivolol improve carotid artery distensibility in hypertensive patients. J Hypertension 1989;7(suppl 6):262-263.

Van Obberghen-Schilling E, Roche NS, Flanders KC, Sporn MB, Roberts AB: Transforming growth factor $B_{1}$ positively regulates its own expression in normal and transformed cells. J Biol Chem 1988;263:7741-7746.

Van de Voorde J, Leusen I: Role of the endothelium in the vasodilator response of rat thoracic aorta to histamine. Eur J Pharmacol 1983;87:113-120.

Van de Voorde J, Leusen I: Endothelium-dependent and independent relaxation effects on aorta preparations of renal hypertensive rats. Arch Int Physiol Biochim 1984;92:P35P36.

Verknelen C.F, Romijn JC, Schroeder FH, van Schalkwijk WP, Splinter TA: Quantitation of polyamines in cultured cells and tissue homogenates by reversed-phase highperformance liquid chromatography of their benzoyl derivates. J Chromat 1988;426:4153.

Vischer P, Beeck H, Voss B: Synthesis, intracellular processing and secretion of thrombospondin in human endothelial cells. Eur J Biochem 1985;153:435-443.

Viswanathan M, Tsutsumi K, Correa FM, Saavedra JM: Changes in expression of angiotensin receptor subtypes in the rat aorta during development. Biochem Biophys Res Commun 1991;179:1361-1367.

Vlodavsky I, Friedman R, Sullivan R, Sasse J, Klagsbrun M: Aortic endothelial cells synthesize basic fibroblast growth factor which remains cell-associated and plateletderived growth factor-like protein which is secreted. J Cell Physiol 1987;131:402-428.

Vogel A, Raines E, Kariya B, Rivest M-J, Ross R: Coordinate control of 3T3 cell proliferation by platelet-derived growth factor and plasma components. Proc Natl Acad Sci USA 1987;75(6):2810-2814.

Walker LN, Bowen-Pope DF, Ross R, Reidy MA: Production of platelet-derived growth factor-like molecules by cultured arterial smooth muscle cells accompanies proliferation after arterial injury. Proc Natl Acad Sci USA 1986;83:7311-7315.

Wallenstein S, Zucker CL, Fleiss JL: Some statistical methods useful in circulation research. Circ Res 1980;47:1-9.

Wamer SJC, Auger KR, Libby P: Human interleukin 1 induces interleukin 1 gene expression in human vascular smooth muscle cells. J Exp Med 1987 ; 165:1316-1331.

Warner SJC, Auger KR, Libby P: Interleukin 1 induces interleukin 1.II. Recombinant human interleukin 1 induces interleukin 1 production by adult human vascular endothelial cells. J Immunology 1987 ; 139(6):1911-1917.

Warshaw DM, Root DT, Halpern W: Effects of antihypertensive drug therapy on the morphology and mechanics of resistance arteries from spontaneously hypertensive rats. Blood Vessels 1980; 17:257-270.

Webb RC: Vascular changes in hypertension: In: Cardiovascular pharmacology (2nd edition) (eds: Antonaccio M). Raven Press, New York, 1984, pp 215-255.

Weinstein R, Stemerman MB, Maciag T: Hormonal requirements for growth of arterial smooth muscle cells in vitro: an endocrine approach to atherosclerosis. Science $1981 ; 212: 818-820$.

Weiss L: Long-term treatment with antihypertensive drugs in spontaneously hypertensive rats (SHR). Effects on blood pressure, survival rate and cardiovascular design. Acta Physio] Scand 1974;91:393-408.

Weiss L, Lundgren Y, Folkow B: Effects of prolonged treatment with adrenergic betareceptor antagonists on blood pressure, cardiovascular design and reactivity in 


\section{REFERENCES}

spontaneously hypertensive rats. Acta Physiol Scand 1974;91: 447-457.

Weiss L, Lundgren Y: Chronic antihypertensive drug treatment in young spontaneously hypertensive rats: effects on arterial blood pressure, cardiovascular reactivity and vascular design. Cardiovasc Res 1978;12:744-751.

Westermark B, Claesson-Welsh L, Heldin C-H: Structural and functional aspects of the receptors for platelet-derived growth factor. Prog Growth Factor Res 1989;1:253-266.

White-Tabor C, Tabor H: Polyamines. Annu Rev Biochem 1984;53:749-790.

Williams LT: Signal transduction by the platelet-derived growth factor receptor. Science 1989;243:1564-1570.

Wilson C, Byrom FB: Renal changes in malignant hypertension. Lancet 1939;i:136-139.

Winkles JA, Friesel R, Burgess WH et al: Human vascular smooth muscle cells both express and respond to heparin-binding growth factor I (endothelial cell growth factor). Proc Natl Acad Sci USA 1987;84:7124-7128.

Winquist RJ, Bunting PB, Baskin EP et al: Decreased endothelium-dependent relaxations in New Zeeland genetic hypertensive rats. J Hypertension 1984;2:536-541.

Wolinsky $\mathrm{H}$, Glagow S: A lamellar unit of aortic medial structure and functions in mammals. Circ Res 1976;20:99-101.

Yamori Y, Tarazi RC, Ooshima A. Effects of beta-receptor-blocking agents on cardiovascular structural changes in spontaneous and noradrenaline-induced hypertension in rats. Clin Sci 1980;59 (suppl):457-460.

Yamori Y, Igawa T, Nara Y, Kihara M, Ooshima A: Characteristics of cultured vascular smooth muscle cells in hypertension (Abstract. Clin Exp Hypertension 1981;3:336.

Yamori Y, Mano M, Nara Y, Hori R: Catecholamine-induced polyploidization in vascular smooth muscle cells. Circulation 1987;75 (supll I):92-95.

Yanagisawa M, Kurihara H, Kimura S, Mitsui Y, Kobayashi M, Watanabe TX, Masaki T: A novel potent vasoconstrictor peptide produced by vascular endothelial cells. Nature $1988 ; 332: 411-415$.

Yanagisawa M, Masaki T: Molecular biology and biochemistry of the endothelins. TiPS 1989;10:374-378.

Yang H, Morton W, Lee RMKW, Kajetanowicz A, Forrest JB: Autoradiographic study of smooth muscle cell proliferation in spontaneously hypertensive rats. Clin Sci $1989 ; 76: 475-478$.

Yarden Y, Ullrich A: Molecular analysis of signal transduction by growth factors. Biochemistry 1988;27(9):3113-3119.

Yoshizumi M, Kurihara H, Morita T, Yamashita T, Oh-hashi Y, Sugiyama T, Takaku F Yanagisawa $M$, Maraki $T$, Yazaki $Y$ : Interleukin-1 increases the production of endothelin-1 by cultured endothelial cells. Biochem Biophys Res Commun 1990;166:324-329.

Zimmerman BG: Actions of angiotensin on adrenergic nerve endings. Fed Proc 1978;37:199202. 


\section{SUMMARY}

Atherosclerosis and hypertension are characterized by structural changes in the arterial wall. During these vascular diseases different types of structural changes occur, and various parts of the circulatory system differ in their susceptibility to modification. The reasons for the different growth responses in these diseases and their regional heterogeneity are not clear. Besides extravascular phenomena (hemodynamics, nervous input, and blood born mitogens) also intravascular phenomena (arterial phenotype, extracellular matrix, and paracrine and autocrine mediators) may be involved.

We investigated changes in DNA synthesis, structure and mechanical activity in isolated arteries during exposure to growth factors. Stimulation of DNA synthesis by continuous exposure of the arterial wall to exogenous growth factors is 1) transient in the media; 2) does not, at least initially, compromise contractile reactivity; 3 ) does not alter gross medial structure; but 4) leads to proliferation of smooth musclelike cells outside the media (chapter 3 ). These findings suggest that the number of smooth muscle cells in the arterial media is maintained constant in the presence of even strong mitogenic stimuli.

To evaluate whether intravascular phenomena contribute to local differences in growth responses of the arterial wall, we evaluated responses to tissue culture in a broad variety of arterial preparations (chapter 4). The data indicate that growth responses of the arterial wall differ quantitatively with the anatomical location and branching order of the vascular segment. In addition to the regional heterogeneity of endothelial effects on mitogenic responses of arterial smooth muscle, this seems to be due to regional differences in susceptibility of arterial smooth muscle to exogenous growth factors. In this respect, we speculate that subsets of growth-resistant and growth-prone arterial smooth muscle cells could be heterogeneously distributed over the arterial tree.

The endothelium influences arterial tone and arterial structure. Endothelium dependent reactivity is altered in established hypertension. We therefore evaluated whether this also applies to endothelial modulation of arterial smooth muscle "growth" (chapter 5). The findings confirm that the endothelium can reduce DNA synthesis in intact arterial smooth muscle. This is not modified by hypertension (young SHR, aorta coarcted WKY, TGR) but is selectively reduced in arteries of adult SHR.

Exposure of isolated arteries to mediators that can be produced by smooth muscle and endothelial cells, showed that DNA synthesis in the arterial media and extramedial cell proliferation are influenced by different factors (chapter 6). The observations further indicate that endothelial modulation of medial DNA synthesis 1) does not seem to involve endothelium-derived prostaglandins, NO or IL-1 and 2) can be blunted by basic fibroblast growth 
factor.

Structural responses (media CSA and DNA synthesis) to elevated pressure, induced by Ang II and PHE infusion in vivo, are heterogeneously distributed over the arterial system of young rats (chapter 8). Besides differences between types of blood vessels, also differences are observed between subtypes of smooth muscle cells within a given arterial segment.

In the last chapter, we describe the long-term actions of Ang II on arterial smooth muscle. The data confirm that Ang II induces an acute transient contractile effect and a hypertrophic effect in arterial smooth muscle. In addition, this peptide induced also a slowly developing tonic increase in wall tension. The mechanism of this long-term tonic increase in tone largely remains to be established, but may involve altered cellular handling of hydrogen ions. These observations suggest that not only structural but also slow functional mechanisms are involved in the development of hypertension.

In summary, observations presented in this thesis suggest that the reasons for the regional heterogeneity of arterial smooth muscle growth responses include 1) the different endothelial modulation of arterial growth responses along the vascular tree; 2 ) the arterial smooth muscle cell subtype composition of the arterial media; and 3) regional heterogeneity of arterial receptor subtypes. 


\section{SAMENVATTING}

Atherosclerose en hypertensie worden gekarakteriseerd door veranderingen in de struktuur van de arteriële vaatwand. De strukturele veranderingen die optreden in de vaatwand en de gebieden in de vaatboom die een verandering hebben ondergaan, zijn verschillend in beide vaatziekten. De redenen voor de verschillende groeiresponsen in deze vaatziekten en hun regionale heterogeniteit zijn niet duidelijk. Naast extravaskulaire fenomenen (hemodynamiek, zenuwinnervatie, en cirkulerende mitogene faktoren) kunnen ook intravaskulaire fenomenen in dit proces betrokken zijn.

In eerste instantie hebben wij de veranderingen in DNA synthese, struktuur en mechanische aktiviteit bestudeerd in geïsoleerde arteriën onder invloed van groeifaktoren (serum). Stimulatie van DNA synthese door exogene groeifaktoren is 1) van voorbijgaande aard in de media; 2) beïnvloedt niet, althans in de beginfase, de kontraktiele reaktiviteit; 3 ) leidt niet tot veranderingen in mediale struktuur; maar 4) resulteert in proliferatie van gladde spiercel-achtige cellen buiten de media (hoofdstuk 3). Deze observaties suggereren dat in aanwezigheid van sterke mitogene stimuli het aantal gladde spiercellen in de arteriële media konstant blijft.

Om na te gaan of intravaskulaire fenomenen bijdragen aan de lokale verschillen in strukturele veranderingen van de vaatwand, hebben wij groeiresponsen bestudeerd in verschillende arteriële preparaten gedurende orgaankweek (hoofdstuk 4). De resultaten geven aan dat groeiresponsen in de arteriële vaatwand kwantitatief verschillen, afhankelijk van de anatomische lokatie en het vertakkingsniveau van het vaskulaire segment. Naast de regionale heterogeniteit van endotheliale effekten op mitogene responsen van de arteriële gladde spier is dit mogelijk een gevolg van regionale verschillen in de gevoeligheid van de arteriële gladde spier voor exogene groeifaktoren. Wij veronderstellen daarom dat in de vaatboom de distributie van subtypen van groei-resistente en groei-gevoelige arteriële gladde spiercellen heterogeen is.

Het endothelium beïnvloedt zowel arteriële tonus als arteriële struktuur. In hypertensie is de endotheel afhankelijke reaktiviteit veranderd. Of dit ook het geval is voor een endotheliale modulatie van arteriële gladde spier "groei" hebben wij nader bestudeerd (hoofdstuk 5). De observaties bevestigen dat het endothelium de DNA synthese reduceert in de intacte arteriële gladde spier. Dit effect wordt niet beïnvloed door hypertensie (jonge spontaan hypertensieve ratten (SHR), aortakoarktatie, transgene ratten (TGR)) maar is selektief verminderd in vaten van volwassen SHR.

Het blootstellen van geïsoleerde arteriën aan mediatoren die geproduceerd kunnen worden door de gladde spier en endotheelcellen, laat zien dat DNA synthese in de media en extramediale celproliferatie beïnvloed worden door verschillende faktoren (hoofdstuk 6). Tevens blijkt dat endothelium- 
afkomstige prostaglandinen, NO of IL-1, niet betrokken zijn in de endotheliale modulatie van mediale DNA synthese. Daarentegen is bFGF in staat de endothelium-afhankelijke reduktie in DNA synthese op te heffen.

Strukturele veranderingen onder invloed van een verhoogde druk, veroorzaakt door infusie van Ang II en PHE in jonge ratten, zijn heterogeen verdeeld over de vaatboom (hoofdstuk 8). Naast verschillen tussen typen bloedvaten treden verschillen op tussen subtypen van gladde spiercellen binnen een arterieel segment.

In het laatste experimentele hoofdstuk worden de lange-termijn effekten van Ang II op de arteriële gladde spier beschreven. De data bevestigen dat Ang II naast akute vasokonstriktie een hypertroof effekt veroorzaakt in de arteriële gladde spier. Bovendien blijkt dit peptide een trage vasokonstriktie te induceren, waarbij een verandering in de cellulaire ion-huishouding (met name $\mathrm{H}^{+}$-ionen) oorzakelijk betrokken zou kunnen zijn. Deze observaties geven aan dat niet alleen strukturele maar ook trage funktionele mechanismen een rol kunnen spelen in het ontstaan van hypertensie.

Samengevat suggereren de resultaten die beschreven zijn in dit proefschrift, dat de regionale heterogeniteit van arteriële groeiresponsen onder andere verklaard kan worden door 1) de verschillen in endotheliale modulatie van arteriële groeiresponsen in de vaatboom; 2) de arteriële gladde spiercel subtype samenstelling van de media; en 3) de regionale heterogeniteit van arteriële receptor subtypen. 


\title{
CURRICULUM VITAE
}

\author{
P.M.H. Schiffers
}

23-05-1957

Geboren te Heerlen

1969-1974

HAVO, Rombouts College te Brunssum

$1974-1978$

HTS (Chemische Technologie) te Heerlen

$1978-1986$

Opleiding Scheikunde, Rijksuniversiteit Utrecht, Utrecht

$1986-1992$

Promovendus, vakgroep Farmacologie, Faculteit der Geneeskunde, Rijksuniversiteit Limburg, Maastricht.

Promotor Prof. dr. K.H. Rahn, 1986-1988, Promotor Prof. dr. H.A.J. Struijker Boudier en co-promotor J.G.R. De Mey, 1988-1992

1992- Universitair Docent, hoofd laboratorium Klinische Farmakologie, vakgroep Farmacologie, Rijksuniversiteit Limburg, Maastricht

\section{LIST OF PUBLICATIONS}

\section{Full Papers}

Boonen HCM, Schiffers PMH, Fazzi GE, Janssen GMJ, Daemen MJAP, De Mey JGR: DNA synthesis in isolated arteries. Kinetics and structural consequences. Am J Physiol 1991;260: $\mathrm{H} 210-\mathrm{H} 217$.

Boonen HCM, Daemen MJAP, Eerdmans PA, Fazzi GE, Janssen GMJ, Kleef EM van, Schiffers PMH, De Mey JGR: Mesenteric resistance artery changes following vasoconstrictor infusion in young rats. J Cardiovase Pharmacol 1993;22:388-395.

De Mey JGR, Uitendaal MP, Boonen HCM, Schiffers PMH, Fazzi GF: Growth responses in isolated elastic, muscular and resistance-sized arterial segments of the rat. Blood vessels 1991:28:372-385.

De Mey JGR, Daemen MJAP, Boonen HCM. Bosman FT, Dijkstra EH, Fazzi GE, Janssen GMJ, Schiffers PMH, Struijker Boudier HAJ, Vrijdag MJJF: In vivo DNA synthesis is not uniformly increased in arterial smooth muscle of young spontaneously hypertensive rats. J Hypertension 1991;9:695-701. 
De Mey JGR, Boonen HCM, Schiffers PMH, Fazzi GE, Janssen GJM: What could underlie hyperplasia of resistance arteries in hypertension? In: Resistance arteries, structure and function. eds. Mulvany et al, Elsevier, Amsterdain, the Netherlands, 1991, pp. 289291.

De Mey JGR, Schiffers PMH: Effects of the endothelium on growth responses in arteries. J Cardiovasc Pharmacol 1993;21 (suppl 1):S22-S25.

Mooij JMV, Schiffers PMH, Rahn KH: Total and free steady-state plasma levels and pharmacokinetics of nifedipine in patients with terminal renal failure. Eur J Clin Pharmacol 1989;37:185-189.

Schiffers PMH, Struijker-Boudier HAJ, De Mey JGR: Effects of angiotensin II and angiotensin converting enzyme inhibitors on contractile and growth responses in isolated carotid arteries of the rat. Basic Res Cardiol 1991;86 (suppl 1):83-89.

Schiffers PMH, Janssen GMJ, Fazzi GE, Struijker-Boudier HAJ, De Mey JGR: Endothelial modulation of DNA synthesis in isolated arteries of the rat. J Cardiovasc Pharmacol 1992;20 (suppl 12):S124-S127.

Schiffers PMH, Van der Heijden HAMD, Fazzi GE, Struijker-Boudier HAJ, De Mey JGR: Tonic tone in arteries exposed continuously to angiotensin II in vitro. J Parmacol Exp Ther 1993;266:1520-1527.

Schiffers PMH, Fazzi GE Janssen GMJ, Uitendaal MP, Struijker-Boudier HAJ, De Mey JGR: DNA synthesis in isolated arteries of normotensive and hypertensive rats: Effects of the endothelium. J Hypertension, submitted.

Schiffers PMH, Fazzi GE, Van Ingen Schenau D, De Mey JGR: Effects of candidate autocrine and paracrine mediators on growth responses in isolated arteries of the rat. Arteriosclerosis and Thrombosis, accepted.

Van Bortel LMAB, Böhm ROB, Mooij JMV, Schiffers PMH, Rahn KH: Total and free steady-state plasma levels and pharmacokinetics of nifedipine in patients with terminal renal failure. Eur J Clin Pharmacol 1989;37:185-189.

Van Bortel LMAB, Böhm ROB, Mooij JMV, Schiffers PMH, Rahn KH: Pharmacokinetics of nitrendipine in terminal renal failure. Eur J Clin Pharmacol 1989;36:457-471.

Van Bortel LMAB, Schiffers PMH, Böhm ROB, Mooij JMV, Rahn KH: The influence of chronic treatment with verapamil on plasma atrial natriuretic peptide levels in young and elderly hypertensive patients. Eur J Clin Pharmacol 1990;39 (suppl 1):39-40.

Van Baak MA, Mooij JMV, Schiffers PMH: Exercise and the pharmacokinetics of propranolol, verapamil and atenolol. Eur J Clin Pharmacol 1992;43:547-550.

Van Hooft IMS, Schiffers PMH, Grobbee DE, Ran KH, Hofman A: Similar plasma atrial natriuretic factor levels in children and young adults with a high and low probability of developing hypertension. The Dutch Hypertension and Offspring Study. J Hypertension 1988;6:S208-S210.

Van Hooft IMS, Schiffers PMH, Grobbee DE, Rahn KH, Hofman A: Similar plasma atrial natriuretic factor levels in children and young adults with a high and low probability of developing hypertension: The Dutch Hypertension and Offspring Study. J Hypertension 1989;7 (suppl 1):43-44.

Van Hooft IMS, Grobbee DE, Hofman A, Schiffers PM, De Pont JJHHM: Atrial natriuretic factor and sodium intake in offspring of hypertensive and normotensive parents. The New Engl J Med 1989;320:867-868.

Van Kleef EM, Fazzi GE, Janssen GMJ, Janssen AJMH, Schiffers PMH, Daemen MJAP, De Mey JGR: Heterogeneity of growth responses in the arterial system of young rats during infusion with phenylephrine and angiotensin II. Submitted. 


\section{Abstracts}

De Mey JGR, Schiffers PMH, Boonen HCM: Proliferating conditions after adrenergic responses in muscular and resistance arterial smooth muscle. J Vasc Biol Med 1989.

De Mey JGR, Schiffers PMH: Regional heterogeneity of arterial growth responses. 3rd International Symposium on Resistance Arteries. Rebild, 1991.

Janssen GMJ, Schiffers PMH, De Mey JGR: Organ culture alters the activity of protein kinase $A$ in isolated renal arteries of the rat. Eur J Pharmacol 1990;183 (3):752.

Schiffers PMH, Van Hooft IMS, Grobbee DE, Rahn KH, Hofman A: Atriales natriuretisches peptide (ANP) bei Kindem von Eltern mit unterschiedlichem Blutdruckniveau. II. Tagung Deutsche Liga zur Bekämpfung des Hohen Blutdruckes, Trier, 1987.

Schiffers PMH, Van Hooft IMS, Grobbee DE, Rahn KH, Hofman A: Atrial natriuretic peptide in children and young adults with different probability of developing hypertension. J Hypertension 1988;6:170.

Schiffers PMH, Van Hooft IMS, Grobbee DE, Rahn KH, Hofman A: Atrial natriuretic peptide (ANP) in children from parents with contrasting blood pressure. J Clin Exp Hypertension 1988;10:722.

Schiffers PMH, Van Bortel LMAB, Struijker-Boudier HAJ: Effects of verapamil on the secretion of atrial natriuretic peptide in young and elderly hypertensive patients. Parmaceut Wkbl Sci Ed 1988;10:233.

Schiffers PMH, Kitslaar P, De Mey JGR: Effects of endothelin and platelet-derived growth factor on contractile reactivity in human omental resistance arteries. Fund Clin Pharmacol 1989;3:430.

Schiffers PMH, De Mey JGR: Effects of endothelin and growth factors on the contractile reactivity in isolated renal muscular and resistance-sized arteries. Naun Schmied Arch Pharmacol 1989;339 (suppl):R65.

Schiffers PMH, PMH, Boonen HCM, Dijkstra EH, Fazzi GE, Daemen MJAP, De Mey JGR: Increased nuclear incorporation of bromo-deoxyuridine in muscular arteries but not in resistance-sized arteries of young spontaneously hypertensive rats in vivo. Pharmaceut Wkbl Sci Ed 1989; suppl J:J10.

Schiffers PMH, De Mey JGR: Effects of inhibitors of angiotensin converting enzyme on growth responses in isolated carotid arteries of the rat. Satellite Symposium to the 11 th IUPHAR Meeting, Maastricht, 1990.

Schiffers PMH, De Mey JGR: Elements of the renin-angiotensin-system in isolated carotid artery of the rat. Eur J Pharmacol 1990;183 (6):2206.

Schiffers PMH, Fazzi GE, Struijker-Boudier HAJ, De Mey JGR: Effects of angiotensin II and endothelin-1 on growth responses in isolated renal artery smooth muscle of the rat. Pharmaceut Wkbl Sci Ed 1990;12 (suppl H):H10.

Schiffers PMH, Fazzi GE, Struijker-Boudier HAJ, De Mey JGR: Effects of interleukin-1 (IL-1) on growth responses in isolated renal artery smooth muscle of the rat. Proceedings 3rd Annual Meeting FEDERA, Amsterdam, 1991.

Schiffers PMH, Janssen GMJ, Fazzi GE, De Mey JGR: Local modulation of growth responses in isolated arteries of adult spontaneously hypertensive rats. Proceedings 7 th International Symposium on SHR and related studies, Lyon, 1991.

Schiffers PMH, Struijker-Boudier HAJ, De Mey JGR: Continuous recording of mechanical activity in isolated arteries during 3 day exposure to angiotensin II. Acta Clin Belg 1991;46:253.

Schiffers PMH, Struijker-Boudier HAJ, De Mey JGR: A "slow pressor" response to 
angiotensin II in vitro. Pharmaceut Wkbl Sci Ed 1991;13 (suppl H):H10.

Schiffers PMH, Janssen GMJ, Fazzi GE, Struijker-Boudier HAJ, De Mey JGR: Bidirectional modulation of DNA synthesis in isolated arteries by the endothelium. Scientific Conference on Functional and Structural Mechanisms of Vascular Control, Snowbird, 1992.

Schiffers PMH, Janssen GMJ, Fazzi GE, Struijker-Boudier HAJ, De Mey JGR: Endothelial modulation of DNA synthesis in isolated arteries of the rat. 2nd International Symposium on Endothelium-derived Vasoactive Factors, Basel, 1992.

Schiffers PMH, Fazzi GE, Janssen GMJ, Struijker-Boudier HAJ, De Mey JGR: Measurement of growth responses in isolated arteries of the rat: Endogenous thymidine, a rate limiting factor? Pharmaceut Wkbl Sci Ed 1992;14 (suppl H):H12.

Schiffers PMH, Van der Heijden HAMD, Struijker-Boudier HAJ, De Mey JGR: Long-term actions of angiotensin II in isolated arteries. 3rd International Symposium on ACE Inhibition, Amsterdam, 1993.

Schiffers PMH, Van der Heijden HAMD, Fazzi GE, Struijker-Boudier HAJ, De Mey JGR: Tonic tone in arteries continuously exposed to angiotensin II in vitro. Pharmacol and Toxicol 1993;72 (suppl II):15.

Schiffers PMH, Fazzi GE, Struijker-Boudier HAJ, De Mey JGR: Endothelium removal increases stimulated DNA synthesis in arteries of normo- and hypertensive rats. IUPS, Glasgow, 1993.

Van Baak MA, Mooij JMV, Schiffers PMH: Plasma concentration changes of antihypertensive drug during dynamic physical exercise. Pharmaceut Wkbl Sci Ed 1988;10:181.

Van Baak MA, Mooij JMV, Schiffers PMH: Pharmacokinetics of antihypertensive drugs during exercise. Med Sci Sports Exercise 1988;20 (suppl):89.

Van Baak MA, Mooij JMV, Schiffers PMH: Veranderingen in plasmaconcentratie van antihypertensieve geneesmiddelen tijdens dynamische lichamelijke inspanning. Ned Tijdschr Geneeskd 1988;132.

Van Baak MA, Schiffers PM, Böhm ROB, Janssen GMJ: Atrial natriuretic peptide during submaximal and maximal exercise in healthy subjects. Can J Sports Sci 1988;13:35P.

Van Bortel LMAB, Böhm ROB, Mooij JMV, Schiffers PMH, Thijssen HHW, Rahn KH: Plasma levels of nifedipine and nitrendipine on terminal renal failure. Pharmaceut Wkbl Sci Ed 1986;8:305.

Van Bortel LMAB, Böhm ROB, Mooij JMV, Schiffers PMH, Rahn KH: The influence of age on the pharmacokinetics of verapamil given orally. Naun Schmied Arch Pharmacol 1987;335 (suppl):R105.

Van Bortel LMAB, Böhm ROB, Mooij JMV, Schiffers PMH, Thijssen HHW, Rahn KH: Steady-state kinetics of nifedipine and nitrendipine in terminal renal failure. Proceedings 8th Dutch Federation Meeting, no 69, 1987.

Van Bortel LMAB, Böhm ROB, Mooij JMV, Schiffers PMH, Rahn KH: Invloed van leeftijd op de plasma spiegels van verapamil. Ned Tijdschr Geneeskd 1987;131:1548.

Van Bortel LMAB, Böhm ROB, Mooij JMV, Schiffers PMH, Rahn KH: Plasma levels of verapamil and age. Pharmaceut Wkbl Sci Ed 1987;9:188.

Van Bortel LMAB, Böhm ROB, Mooij JMV, Schiffers PMH, Thijssen HHW, Rahn KH: Plasmaspiegels van nifedipine en nitrendipine bij terminale nierinsufficientie. Ned Tijdsch Geneeskd 1987;131:1369.

Van Bortel LMAB, Schiffers PMH, Mooij JMV: Invasive hemodynamics of nebivolol in hypertensive patients. Proceedings Intemational Symposium on Cardiovascular Disease and Beta-adrenoceptor Function, Antwerp, 1988. 
Van Bortel LMAB, Böhm R.OB, Mooij JMV, Schiffers PMH, Thijssen HHW, Rahn KH: Steady-state plasma levels of nifedipine and nitrendipine in terminal renal failure. Proceedings XIX Congress of the International Union of Therapeutics, 1989.

Van Bortel LMAB, Schiffers PMH, Böhm ROB, Mooij JMV, Rahn KH, Struijker-Boudier HAJ: Verapamil alters plasma levels in hypertensive patients. Eur J Clin Pharmacol 1989;36 (suppl):A339.

Van Bortel LMAB, Schiffers PMH, Böhm ROB, Mooij JMV, Rahn KH, Struijker-Boudier HAJ: The influence of a chronic treatment with verapamil on plasma human ANP levels in young and elderly hypertensive patients. Abstractbook 4th European Meeting on Hypertension 1989;93.

Van Bortel LMAB, Schiffers PMH, Böhm ROB, Mooij JMV, Rahn KH, Struijker-Boudier HAJ: Antihypertensive treatment with verapamil raises plasma ANP levels in man. Pharmceut Wkbl Sci Ed 1989;11(suppl H):H6.

Van Bortel LMAB, Schiffers PMH, Böhm ROB, Mooij JMV, Rahn KH, Struijker-Boudier HAJ: Verapamil alters plasma human ANP levels in hypertensive patients. Proceedings 31th Dutch FEDERA Meeting 1990;76.

Van Bortel LMAB, Schiffers PMH, Böhm ROB, Mooij JMV, Rahn KH, Struijker-Boudier HAJ: Plasma ANP levels during hypertensive treatment with verapamil. Netl J Med 1990;37 (4):A37.

Van Bortel LMAB, Schiffers PMH, Mooij JMV: Invasive haemodynamics of nebivolol in hypertensive patients. Drug Invest 1991;3 (supp] 1):60.

Van Hooft IMS, Schiffers PMH, Grobbee DE, Rahn KH, Hofman A: Similar plasma atrial natriuretic peptide (ANP) levels in children and young adults with a high and low probability to develop hypertension. Proceedings of 12th Scientific Meeting of the Intemational Society of Hypertension, Kyoto, 1988.

Van Hooft IMS, Schiffers PMH, Grobbee DE, Hofman A: Adrenoceptors in youngsters with a different probability to develop hypertension. 2nd Int Conf on Preventive Cardiology and the 29th Annual Meeting AHA Council on Epidemiology. Proceedings, Washington, 1989.

Van Hooft IMS, Grobbee DE, Schiffers PMH, Boomsma F, Hofman A: Raised alpha-2 receptor density with normal beta-2 receptor density in youngsters at risk for hypertension: the Dutch hypertension and offspring study. 4th European Meeting on Hypertension. Proceedings, Milaan, 1989. 


\section{DANKWOORD}

In de eerste plaats wil ik mijn promotor Harry Struijker Boudier bedanken voor het feit dat ik, na het vertrek van Prof. dr. K.H. Rahn naar Münster, in de gelegenheid werd gesteld een nieuw promotie onderzoek te starten op het laboratorium van Jo De Mey. Vanaf die tijd heb je op de achtergrond, maar steeds nauwlettend, mijn werk gevolgd en heb je mijn proefschrift in de afrondingsfase voorzien van kritische kanttekeningen en waardevolle suggesties.

Werken op het laboratorium van mijn co-promotor Jo De Mey was en is wetenschappelijk gezien een ware belevenis. Je komt daarbij in aanraking met alle facetten die te maken hebben met de biologie en de pathologie van de vaatwand. Jo, ik wil jou bedanken voor de enorme bijdrage die jij hebt geleverd aan mijn promotieonderzoek en het tot stand komen van mijn proefschrift. Tevens wil ik jou maar ook je vrouw bedanken voor jullie gastvrijheid tijdens menig uurtje van gezellig samen zijn.

Zoals gezegd, niet alleen wetenschappelijk maar ook sociaal gezien is het gezelschap "JO \& CO" uniek. Ik wil daarom alle CO's bedanken voor de professionele assistentie die ik bij talloze experimenten heb gehad en de fijne gezellige tijd die ik op het lab en natuurlijk ook daarbuiten heb meegemaakt.

Jet en Geertje, jullie wil ik bedanken voor de prettige samenwerking op het lab klinische farmakologie.

Een woord van dank gaat ook uit naar alle leden van de vakgroep Farmakologie voor hun aangename kollegialiteit.

Tenslotte bedank ik de leden van de beoordelingscommissie: Prof. dr. P.W. de Leeuw, Dr. M.J.A.P. Daemen, Prof. dr. A.C. Grittenberger-de Groot, Prof. dr. A.M. Heagerty en Prof. dr. P.J.A.M. Kitslaar voor de kritische beschouwing en de positieve beoordeling van mijn proefschrift. 
